\title{
Mica as an Ultra-Flat Substrate for Studying Mechanically Exfoliated Graphene
}

By

Mashael Mohammed Alshaikh

Thesis submitted to Swansea University in fulfilment of the requirements for the Degree of Doctor of Philosophy 


\section{Abstract}

Silicon dioxide $\left(\mathrm{SiO}_{2}\right)$ is a common support for studying two-dimensional materials and creating devices from them. However, graphene conformation to $\mathrm{SiO}_{2}$ roughness worsens the electronic properties, whereas graphene deposited on flat terraces of insulating mica is free of ripples. This thesis solves key challenges in the use of mica to support mechanically exfoliated graphene.

Methods of mica cleavage and graphene exfoliation, and settings for electron microscopy, atomic force microscopy (AFM) and Raman spectroscopy were developed.

Vacuum annealing was compared for graphene samples of different thicknesses, down to a single layer. Pre- and post-annealing, graphene on mica provided defect-free graphene and no observable strain or doping. In contrast, graphene on $\mathrm{SiO}_{2}$ showed disorder before annealing. Annealing up to $300^{\circ} \mathrm{C}$ reduced the Raman defect peak but did not remove it. Above $300^{\circ} \mathrm{C}$, the defect peak increased.

Graphene on $\mathrm{SiO}_{2}$ appeared to become 'invisible' with AFM after annealing at $500^{\circ} \mathrm{C}$, in line with previous observations with scanning electron microscopy. Other studies attributed this to the graphene being removed, but, here, using substrate markers, Raman spectroscopy and line-averaged AFM showed that the graphene was still present but had conformed to the underlying roughness of the $\mathrm{SiO}_{2}$ so well as to appear nearly invisible.

Mica annealed at $400^{\circ} \mathrm{C}$ showed the formation of potassium carbonate particles following dehydroxylation of the mica surface at a temperature lower than previously reported. In addition, the graphene appeared to act as a mask, protecting the mica underneath it while the surrounding surface was removed at $500^{\circ} \mathrm{C}$.

Patterning and etching mica are essential to create location grids and etch trenches to suspend deposited materials. The first patterning lithography recipe for mica was established herein using electron-beam lithography. Finally, mechanically exfoliated graphene was successfully transferred to the patterned mica and studied. 


\section{Declaration and Statements}

This work has not previously been accepted in substance for any degree and is not being concurrently submitted in candidature for any degree.

Signed

Date 22-02-2021

\section{STATEMENT 1}

This thesis is the result of my own work and investigation, except where otherwise stated. Other sources are acknowledged by footnotes giving explicit references. A bibliography is appended.

Signed

Date 22-02-2021

\section{STATEMENT 2}

I hereby give consent for my thesis, if accepted, to be available for photocopying and for inter-library loan, and for the title and summary to be made available to outside organisations.

Signed

Date 22-02-2021 


\section{Contents}

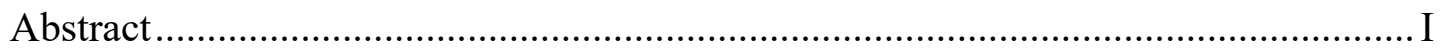

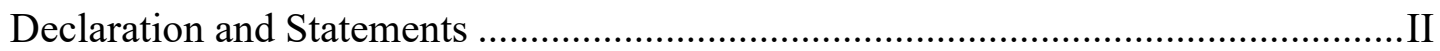

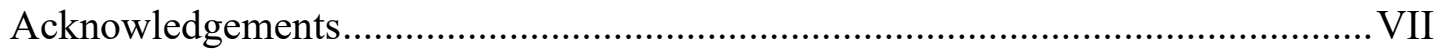

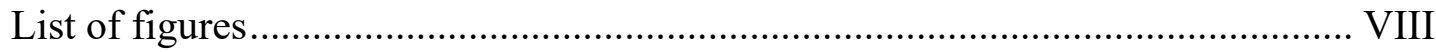

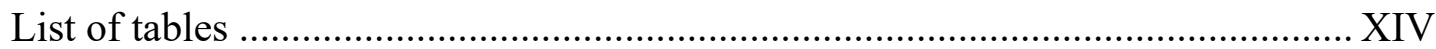

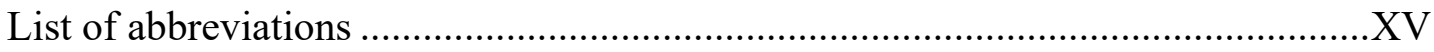

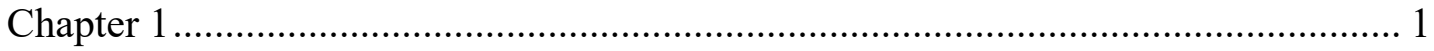

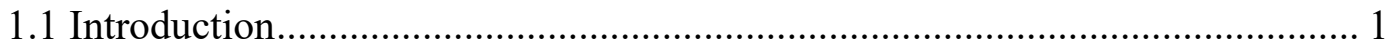

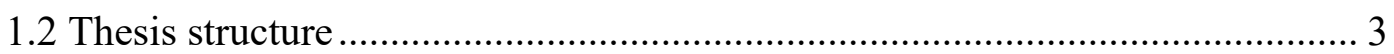

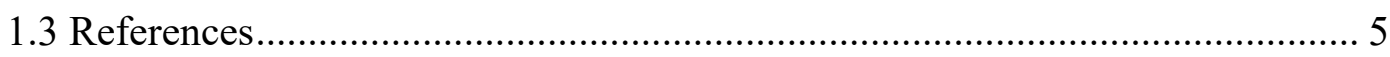

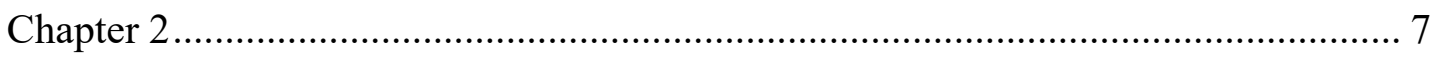

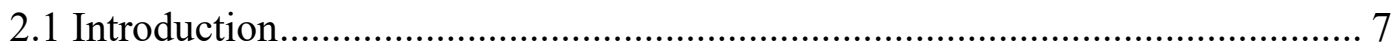

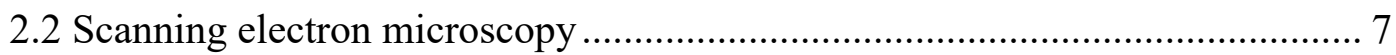

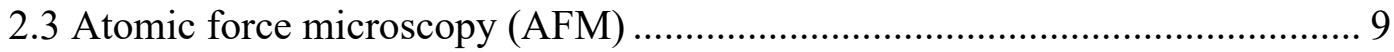

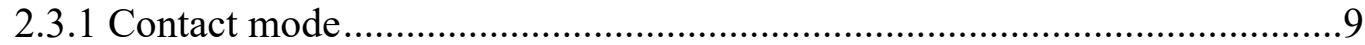

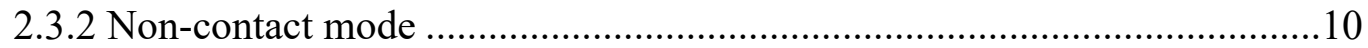

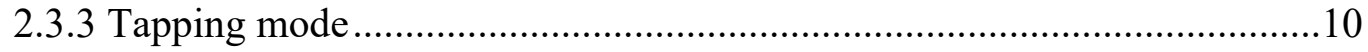

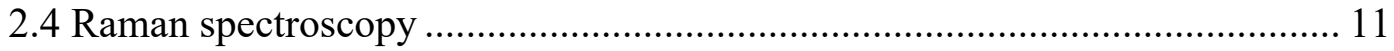

2.4.1 Raman spectra for graphene .............................................................13

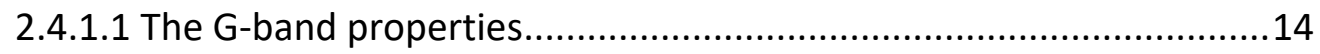

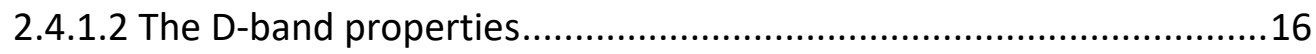

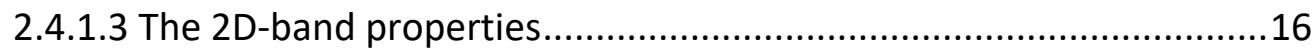

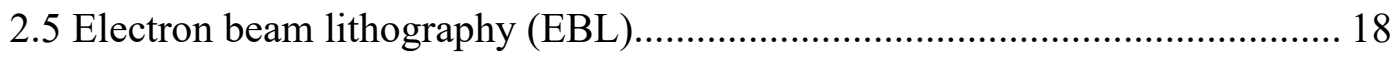

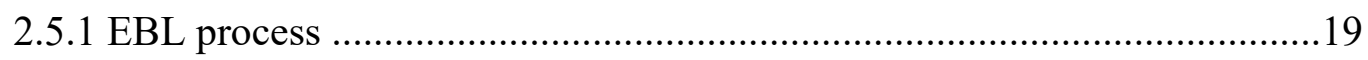

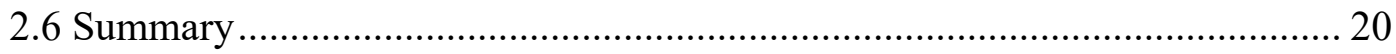

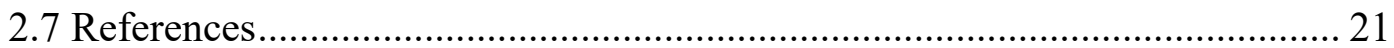




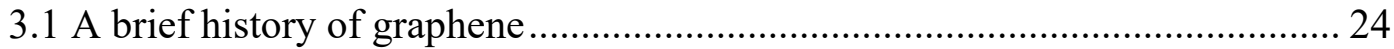

3.2 Graphene properties: Structural properties ............................................... 24

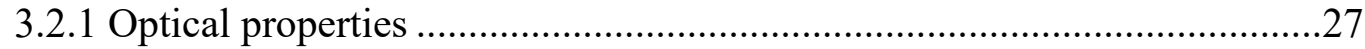

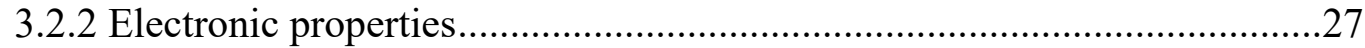

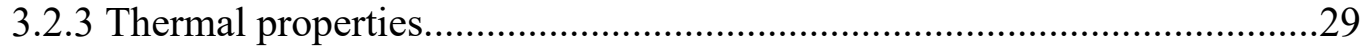

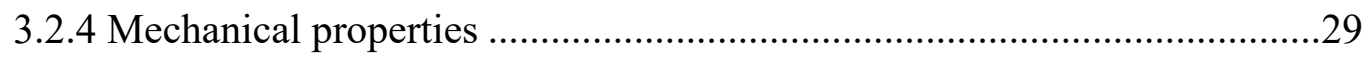

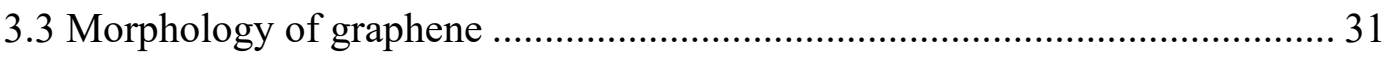

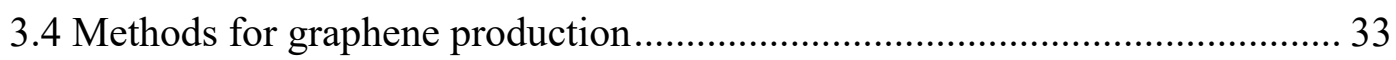

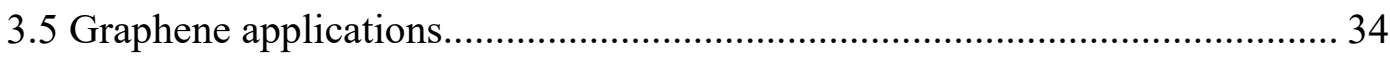

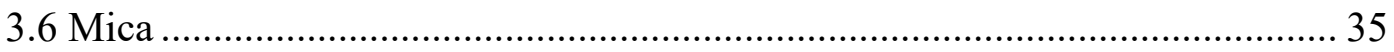

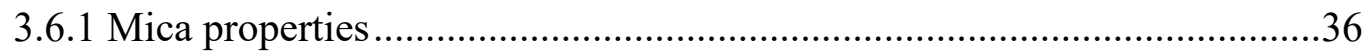

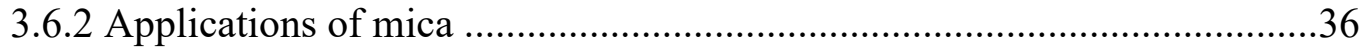

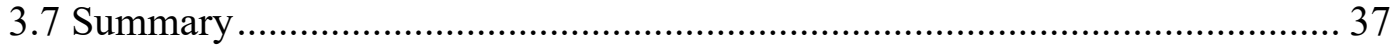

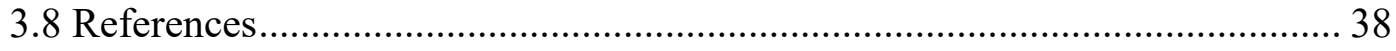

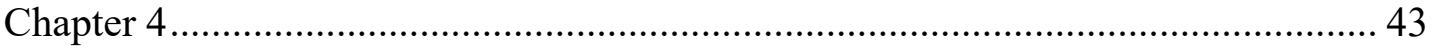

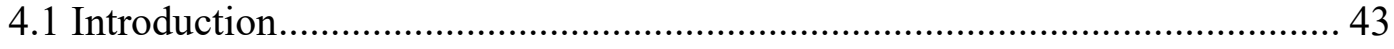

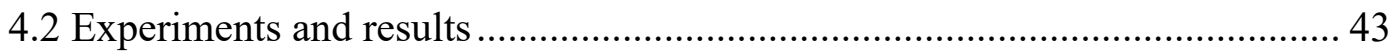

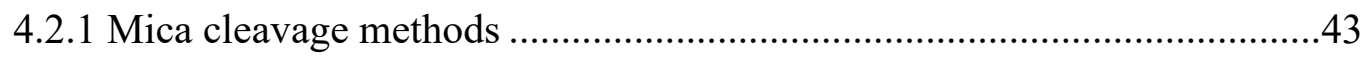

4.2.2 Graphene deposition methods on substrate ...........................................46

4.2.3 Graphene on mica preparation environments ........................................49

4.2.4 Different characterisation techniques for the same area.........................50

4.2.5 Developing SEM techniques to image graphene on $\mathrm{SiO}_{2}$ and mica ..........51

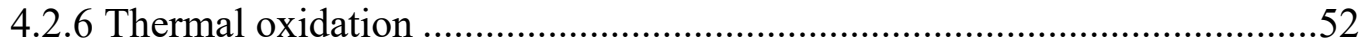

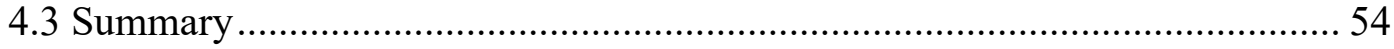

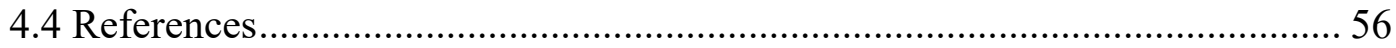

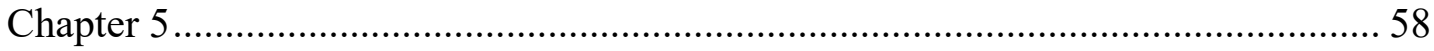


5.1 Introduction

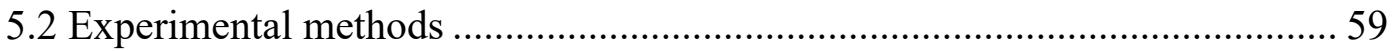

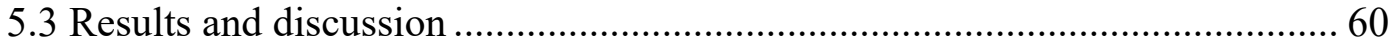

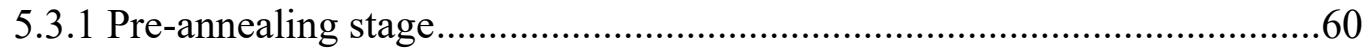

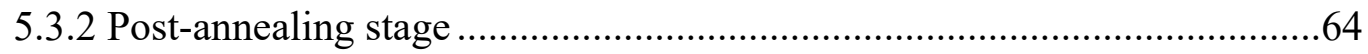

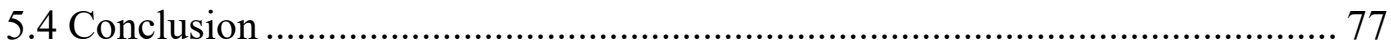

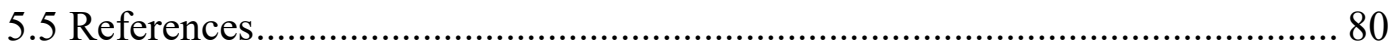

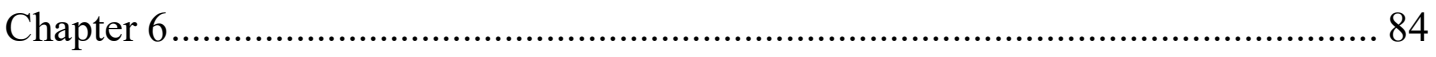

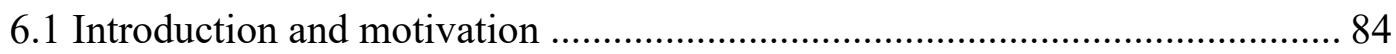

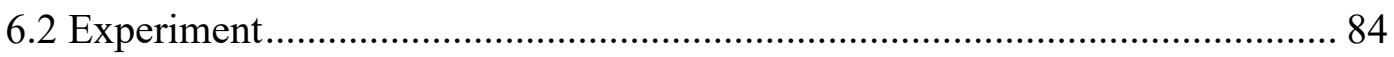

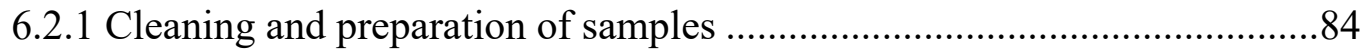

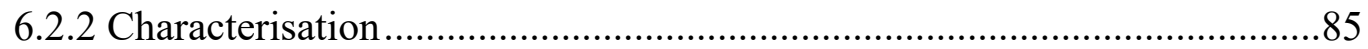

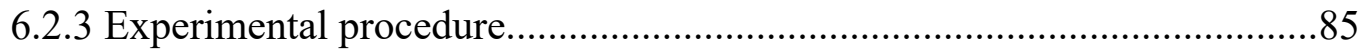

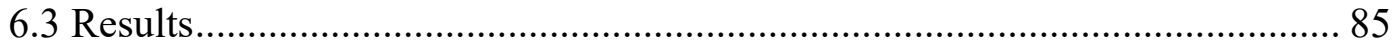

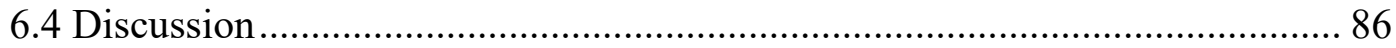

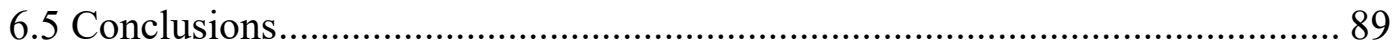

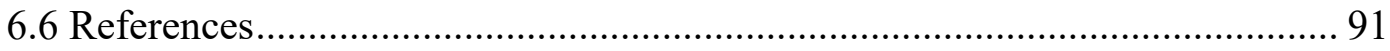

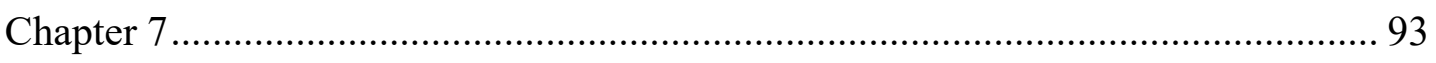

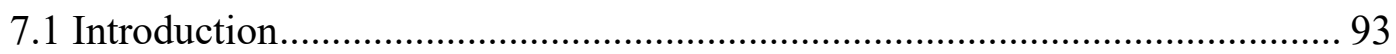

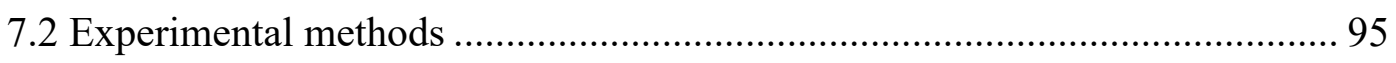

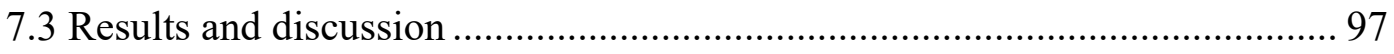

7.3.1 The problem of the adhesion between the mica and the resist layer or any

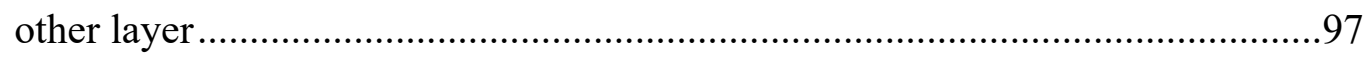

7.3.2 The problem of pattern invisibility under optical microscopy .................101

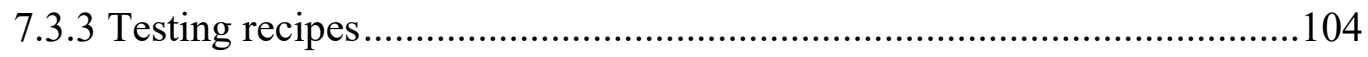

A: Lithography process steps for the first recipe ...................................104

B: Ultraviolet (UV) photolithography process steps for the second recipe.112

C: Final lithography process....................................................................120 
7.3.4 Suspended graphene on mica substrate

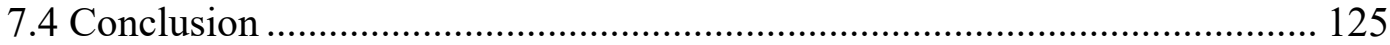

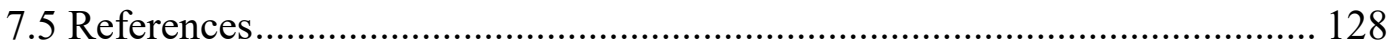

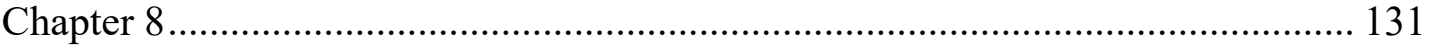

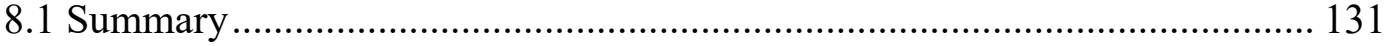

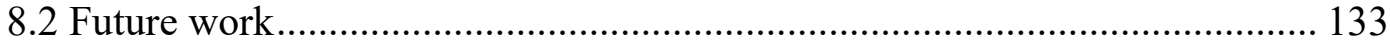




\section{Acknowledgements}

I thank Almighty Allah for giving me the opportunity and strength to undertake and complete this research with His continuous mercy and grace.

I would like to thank and express my great gratitude and appreciation to my supervisor, Dr Richard Cobley, for his guidance, support and encouragement throughout the entire period of this project.

I am grateful to Saudi Scholarship (Umm-Al Qura University, Kingdom of Saudi Arabia) for their financial support and continuous assistance during my academic degree.

I would also like to thank my parents, my husband and my children, who have been waiting eagerly for this moment. I could not have done this degree without your prayers, unlimited patience and endless love. I will never stop appreciating your constant support. 


\section{List of figures}

Figure 2.1: Schematic representation of the basic components of an SEM microscopy ....................................................................................................................... 8 8

Figure 2.2: Schematic representation of the main principles of AFM................. 9

Figure 2.3: Schematic representation of (a) Rayleigh and Raman scattering, and (b) the resulting Raman spectrum [11].

Figure 2.4: Schematic representation of the Raman spectroscopy components

Figure 2.5: Raman signatures from several types of nanocarbon material. From bottom to top : amorphous carbon, single-wall carbon nanohorns (SWNH) , damaged graphene, a single-wall carbon nanotube (SWNT), highly oriented pyrolytic graphite (HOPG) and crystalline monolayer graphene. The term G' band in this figure is referred to as the $2 \mathrm{D}$ band in this thesis $[18]$.

Figure 2.6: The G-band split into $\mathrm{G}^{-}$and $\mathrm{G}^{+}$. As the strain increases, the splitting increases [19].

Figure 2.7: Raman spectra to determine the number of layers in graphene based on a) Shape of 2D and intensity $I_{2 D} / I_{G}$, and b) FWHM of the 2D band [20].

Figure 2.8: Raman spectra to determine the number of layers in graphene based on an intensity ratio [21].

Figure 2.9: A schematic illustration of the EBL process for a negative e-resist. 20

Figure 3.1: (a) Honeycomb lattice of monolayer graphene, where white (black) circles indicate carbon atoms on A (B) sites, and (b) the reciprocal lattice of monolayer graphene, where the shaded hexagon is the corresponding Brillouin zone (c) Carbon atom structure. (d) Energy levels of outer electrons in carbon atoms. (e) The production of $\mathrm{sp}^{2}$ hybrids [4].

Figure 3.2: $\mathrm{Sp}^{2}$ carbon materials [6]. 27

Figure 3.3: Sigma and pi bonds formed by $\mathrm{sp}^{2}$ hybridisation .[4] 28 
Figure 3.4: band structure of pristine graphene with two cones at a Dirac point [11] 28

Figure 3.5: a) TEM image of suspended graphene membranes. (b)(c) Electron diffraction patterns from a graphene single layer under different incidence angle of 0 (b) and 26 (c) degrees. (d) Schematic for corrugated graphene (e) Flat graphene crystal [15]. 31

Figure 3.6: Graphene roughness on a) $\mathrm{SiO}_{2}$ b) mica substrates [28] 32

Figure 3.7: Left: Graphene synthesis via mechanical exfoliation. [1]. Right: several methods of graphene synthesis $[10]$

Figure 3.8: Graphene applications in various fields [10]. 35

Figure 3.9: Crystal structure of muscovite mica [40] 36

Figure 4.1: Optical microscopy images of mica before and after cleavage by razor blade and adhesive tape. Magnification: $x 10$ and $x 50$.

Figure 4.2: Optical microscopy images of different magnification. a) and b): water drops on cleaved mica after several weeks; c) and d): flat area in the same water sample. Magnification: x10 (left) and x50 (right). 46

Figure 4.3: Optical microscopy (magnification: x50) of graphene on mica using different graphene production methods: a) adhesive tape method, b) tapefree method. 48

Figure 4.4: The same region under different characterisation techniques: a) optical microscopy with image size $60 \mu \mathrm{m}$, b) AFM-contact mode with scan size $50 \mu \mathrm{m}$, and c) SEM.

Figure 4.5: SEM image at different settings. Left: graphene on $\mathrm{SiO}_{2} / \mathrm{Si}$; right: graphene on mica.

Figure 4.6: Thermal oxidation process.

Figure 4.7: $\mathrm{SiO}_{2}$ thickness (measured using ellipsometry) as a function of oxidation time, fitted using a least-squares method.

Figure 5.1: SEM image pre-annealing: a) 'thick' $S 1$ and 'thin' S2 graphene attached to $\mathrm{SiO}_{2} / \mathrm{Si}$ substrate and b) 'thick' $\mathrm{M} 1$ and 'thin' $\mathrm{M} 2$ graphene on mica substrate. 
Figure 5.2: Raman spectra (normalised by the G-band intensity.)of preannealing 'thick' S1 and M1 and 'thin' S2 and M2 regions of the graphene attached to the $\mathrm{SiO}_{2} / \mathrm{Si}$ and mica substrates.

Figure 5.3: $\mathrm{I}_{D} / \mathrm{I}_{G}$ ratio of 'thick' (S1) and 'thin' (S2) graphene on $\mathrm{SiO}_{2}$. Dashed lines guide the eye.

Figure 5.4: Raman spectra of samples (a) $\mathrm{S} 1$ and (b) $\mathrm{S} 2$ (graphene on $\mathrm{SiO}_{2} / \mathrm{Si}$ ), and (c) M1 and (d) M2 (graphene on mica). Spectra (normalised by the Gband intensity ) from bottom to top, pre-annealing and post-annealing, at $200^{\circ} \mathrm{C}, 300^{\circ} \mathrm{C}, 400^{\circ} \mathrm{C}$ and $500^{\circ} \mathrm{C}$, respectively. 66

Figure 5.5: Position of a) G and b) 2D bands for thick graphene (S1 and M1) and thin graphene (S2 and M2) at different annealing temperatures.

Figure 5.6: FWHM of a) G and b) 2D bands for thick (S1 and M1) and thin (S2 and M2) graphene at different annealing temperatures.

Figure 5.7: AFM images of (a) Sample $\mathrm{S} 1$ and $\mathrm{S} 2$ for graphene on $\mathrm{SiO}_{2}$ before annealing and b) after annealing at $500^{\circ} \mathrm{C}$.

Figure 5.8: AFM images of (a) Sample M1 and M2 for graphene on mica before annealing and b) after annealing at $500^{\circ} \mathrm{C}$. It is important to mention that the same settings and mode of AFM were used before annealing and afterwards.

Figure 6.1: AFM images of bare mica substrate before and after the annealing process. Scan size $10 \mu \mathrm{m} \times 10 \mu \mathrm{m}$, all on the same height scale.

Figure 7.1: Mechanical etching of mica substrate using AFM tip. Controlling the hole depth by tuning the scanning times: a) one, b) three, and c) five scan times.

Figure 7.2: Patterning mica procedures 97

Figure 7.3: Optical microscopy image of silicon/nLOF 2070 resist by e-beam lithography with four different exposure doses a) 20, b) 30, c) 40 and d) 50 $\mu \mathrm{C} / \mathrm{cm}^{2}$

Figure 7.4: Optical microscopy image of mica/Cr/nLOF 2070 resist. The pattern started peeling off after a few seconds in the developer solvent. 99 
Figure 7.5: Optical microscopy image of S1 (mica/PMMA/Cr). Left: the pattern is invisible under the normal settings of optical microscopy. Right: The pattern shown after optimising the contrast

Figure 7.6: Optical microscopy image of the lithography of the patterns in the mica in different prepration processes coated the sample with two layers. Left: S1 (mica/PMMA/Cr). Right: S2 (mica/Cr/PMMA) 103

Figure 7.7: Optical microscopy image of sample (mica/PMMA/nlof2070) with exposure dose $100 \mu \mathrm{C} / \mathrm{cm}^{2}$ at different magnifications 106

Figure 7.8: Optical microscopy image of samples (mica/PMMA/nlof2070) with exposure dose (left) 20 and (right) $50 \mu \mathrm{C} / \mathrm{cm}^{2}$

Figure 7.9: Optical microscopy image of EBL (silicon/PMMA/nLOF 2070), magnification $\mathbf{x 3 0 0}$

Figure 7.10: Scheme of lithography and etching process (mica/PMMA/Cr/nLOF) 108

Figure 7.11: Optical microscopy sample of mica/PMMA/Cr/nLOF. Left: S1, maximum dose of $20 \mu \mathrm{C} / \mathrm{cm}^{2}$. Right: $\mathrm{S2}$, maximum dose of $50 \mu \mathrm{C} / \mathrm{cm}^{2}$. The steps within the pattern were produced when the mica was cleaved, as discussed.

Figure 7.12: Optical microscopy image of sample (mica/PMMA/Cr/nLOF).

Developing time: left: $30 \mathrm{~s}$, right: $60 \mathrm{~s}$. The erosion in the pattern increased with the increasing development time. Again note the step on the pattern is produced from the mica cleavage 110

Figure 7.13: Optical microscopy image (mica/PMMA/Cr/nLOF) of baking time of two minutes for both the soft and hard bakes. Developing time: one minute for exposure dose $20 \mu \mathrm{C} / \mathrm{cm}^{2}$

Figure 7.14: Scheme of patterning process of mica/Cr/negative resist $\mathrm{nLOF}$ 2070 using UV exposure.

Figure 7.15: Optical microscopy image of mica/Cr/resist. Left: mica after dry plasma stage. Right: mica after PR stripper DMSO for four hours 
Figure 7.16: Optical microscopy image of mica/Cr/resist Left: mica after stripper for one hour. Right: mica after dry etching.

Figure 7.17: Optical microscopy image (mica/Cr/nLOF 2070) of a) after development and $\mathrm{Cr}$ etching, b) after PR stripping, c) after plasma etching, and d) after stripping the $\mathrm{Cr}$ layer. The steps on the pattern produced from mica cleavage 116

Figure 7.18: (Left) SEM image of shallow-etched mica surface prepared by mica/25 nm Cr/nLOF 2070). The upper part of the image is unetched, the horizontal line is the sidewall, and below that the trench of the etched line. (Right) The same sample with optical microscopy; the dashed line shows the orientation of the faint stripes, which are the repeated stripes just visible. The curved lines are cleave-induced steps.

Figure 7.19: Optical microscopy image of mica/70 $\mathrm{nm}$ Cr/nLOF 2070. Left: the pattern after dry etching. Right: the etched mica of $10 \mathrm{~nm}$ after stripping of all the coated layers. The dashed line shows an example to guide the eye to an etched trench in the mica

Figure 7. 20: Left: Optical image of (mica/250 $\mathrm{nm}$ Cr/nLOF) after RIE. Right) AFM measurement of the etched mica corresponding to the line scan indicated on the optical image

Figure 7.21: Left) Optical microscopy image (mica/250 $\mathrm{nm} \mathrm{Cr} / \mathrm{nLOF}$ ) of etched mica substrate. The increased depth provides more optical contrast. Right) AFM profile of the etched mica as the line scan ion the optical image..... 119

Figure 7.22: Optical microscopy image of patterning EBL for mica substrate. Sample of mica/250 $\mathrm{nm}$ Cr/nLOF 2070. a) Pattern drawn with software (dose of dark blue is 20 , light blue is 30 , green is 40 , red is $50, \mu \mathrm{C} / \mathrm{cm}^{2}$ ). b) After developing for 30 seconds. c) After developing for one minute. d) Post RIE. e) and f) Different areas of etched mica after stripping of all the layers

Figure 7.23: AFM images for mica patterned at different exposure doses a) 20, b) 30 , c) 40 and d) $50 \mu \mathrm{C} / \mathrm{cm}^{2}$ 122 
Figure 7. 24:AFM profile for mica patterned at different exposure doses a) 20,

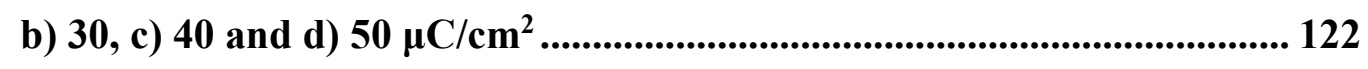

Figure 7.25: Optical image of patterned mica substrate at different

magnifications, indicated with arrows as the fainter lines. Note, the wavy

steps on the pattern are produced from mica cleavage.

Figure 7.26: AFM image for etched mica with a depth of $35 \mathrm{~nm}$. Sample of mica/250 nm Cr/nLOF 2070. Exposure dose $40 \mu \mathrm{C} / \mathrm{cm}^{2}$

Figure 7.27: Suspended graphene on patterned mica substrate. Left: Optical image. Right: SEM..

Figure 7.28: AFM image of suspended graphene sheet on the patterned mica substrate 


\section{List of tables}

Table 3.1: Mechanical properties of graphene [1] .................................................. 30

Table 3.2: Effect on various factors on the Raman peak, shape, and splitting [1].

Table 5.1: Values of position of $G$ band before annealing of graphene sheets and change in frequency of $\mathrm{G}$ band after annealing at $500^{\circ} \mathrm{C}$, for thin and thick graphene on $\mathrm{SiO}_{2}$ and mica substrates. 69

Table 7.1: Test the adhesion of mica /Cr $100 \mathrm{~nm}$ using different treatment.... 100 


\section{List of abbreviations}

\begin{tabular}{|c|c|}
\hline$(\mathrm{ZnO})_{1-\mathrm{x}}(\mathrm{GaN})_{\mathrm{x}}$ & $\begin{array}{l}\text { Alloying zinc oxide and Gallium nitride in films with } \mathrm{x} \text {, where } \\
\mathrm{x} \text { is parameter (dimensionless) predicted by Vegard's law }\end{array}$ \\
\hline AFM & Atomic force microscopy \\
\hline $\mathrm{Al}$ & Aluminium \\
\hline Al NPs & Aluminium nanoparticles \\
\hline $\mathrm{Ar}$ & Argon \\
\hline $\mathrm{Au}$ & Gold \\
\hline CF4 & Tetrafluoromethane \\
\hline $\mathrm{Cr}$ & Chromium \\
\hline CVD & Chemical vapour deposition \\
\hline DI & Deionised \\
\hline DMSO & Dimethyl sulfoxide \\
\hline EBL & Electron beam lithography \\
\hline FEG & Field emission gun \\
\hline FWHM & Full width at half maximum \\
\hline HOPG & Highly oriented pyrolytic graphite \\
\hline $\mathrm{I}(2 \mathrm{D})$ & 2D-band intensity \\
\hline $\mathrm{I}(\mathrm{D})$ & D-band intensity \\
\hline $\mathrm{I}(\mathrm{G})$ & G-band intensity \\
\hline IPA & Isopropyl alcohol \\
\hline MIBK & Methyl isobutyl ketone \\
\hline $\mathrm{N}_{2}$ & Nitrogen \\
\hline NC-AFM & Non-contact atomic force microscopy \\
\hline $\mathrm{nm}$ & Nanometre \\
\hline
\end{tabular}




\begin{tabular}{|l|l|}
\hline $\mathrm{O}_{2}$ & Oxygen \\
\hline PEB & Post-exposure bake \\
\hline PL & Patterning Lithography \\
\hline PMMA & Poly (methyl methacrylate) \\
\hline RF & Radio frequency \\
\hline Sccm & Reactive Ion Etcher \\
\hline SEM & Standard cubic centimeters per minute \\
\hline Si & Scanning electron microscopy \\
\hline SiC & Silicon \\
\hline SiO2 & Silicon carbide \\
\hline TEM & Silicon dioxide \\
\hline UHV & Transmission electron microscopy \\
\hline vdW & Ultra-high vacuum \\
\hline XRD & Van der Waals \\
\hline
\end{tabular}




\section{Chapter 1}

\subsection{Introduction}

To study the physical properties of nanomaterials, they must be deposited onto a substrate material, barring a few exceptions. The substrate may be appropriate for utilising the properties of the nanomaterial in a device or be an idealised substrate for measurement purposes. In both cases, the substrate must structurally support the material over repeated investigations.

Ideally, the substrate would not alter the properties of the nanomaterial, however, this is rarely the case. For example, the effect of growing aluminium nanoparticles ( $\mathrm{Al}$ NPs) using Electron-Beam Physical Vapor Deposition (e-beam PVD), then performing thermal annealing on substrates of indium tin oxide (ITO) and silicon ( $\mathrm{Si}$ ), has been investigated [1]. In this study AFM topography images of annealed aluminium films on Si substrates presented a formation of aluminium nanoparticles, contrary to the aluminium films on indium tin oxide substrates where non-uniform flakes such as aluminium nanostructures were observed. With similar thermal annealing applied to these two substrates, formation of Al NPs appeared uniformly on the silicon due to better adhesion, whereas Al tended to reject adhesion with indium tin oxide and melt on the surface. It is assumed that there is more interaction between Al NPs and Si substrates because the interacting materials tend to decrease the free surface energy, leading to uniform formation of the Al NPs. Therefore, different surface energies of the substrates used affect the nanomaterials differently.

In another example, the structural properties of magnetron sputtered films of alloyed zinc oxide and gallium nitride $(\mathrm{ZnO})_{1-\mathrm{x}}(\mathrm{GaN})_{\mathrm{x}}$ deposited on different substrates of sapphire, zinc oxide and silicon oxide were investigated as a function of strain accumulation and post-deposition annealing [2]. Structurally, the thin alloy films that were deposited on quartz and c-sapphire substrates yielded the highest tensile strain compared to the films deposited zinc oxide. The alloy films were deposited on the csapphire, zinc oxide (O-faced) and quartz substrates, with a lattice-mismatched, lattice-matched and amorphous substrate, respectively. The films were also annealed after deposition to study the effects of thermal treatment. During the annealing process, the overall quality of crystalline films was found to increase and lower the tensile strain 
of the films. This result indicated that the tensile strain in the films strongly depends on the type of substrate used.

Moreover, the nanostructure and preferential orientation are sensitive to the substrate surface. For example, non-conductive films of cadmium telluride nanoparticles (CdTe NPs) were deposited on crystal quartz and amorphous glass, on transparent conducting films of fluorine-doped tin oxide and indium tin oxide (ITO), and a metal substrate of silver. [3] The grain size of preferential orientation changed from 111 to 220 for nonconducting substrates and conducting substrates, respectively. In addition, the energy band gap results of CdTe thin films indicated the impact of the substrates' conductivity on the increase of the direct energy band gap.

In traditional semiconductor devices $\mathrm{SiO}_{2}$ is the most-used dielectric and has typically been used as insulating support for studying the electrical properties of graphene [4]. When graphene is deposited on to patterned $\mathrm{Si} / \mathrm{SiO}_{2}$ substrates, it is always annealed before vacuum investigation to remove contamination. However, our findings showed that this annealing step causes the graphene on the $\mathrm{SiO}_{2}$ to conform structurally to the underlying roughness, altering its appearance in scanning electron microscopy and the disorder measured with Raman microscopy [5]. In addition, graphene fabricated on $\mathrm{SiO}_{2}$ substrates demonstrated worse electrical performance than theoretical predications, due to charge carrier scattering from defects and substrate surface roughness [6-8]. This has driven the interest in alternative substrates for the electrical characterisation of nanomaterials [9-11].

To repeatedly study the same nanostructure among different instruments, it must be possible to locate the same structure across the length scales used by different instruments. Typically, substrates are patterned with guidelines and index markers to achieve this. Substrates could also be patterned to create voids and cavities over which nanomaterials can be suspended to assess vibrational properties or deflection characteristics thereof.

All of these complimentary, and occasionally competing, requirements place significant demands on fabricating substrates suitable for the study of nanomaterials. This has led to the search for alternative support materials including mica. The properties of mica are discussed later in chapter three, but briefly, they relate to an aluminosilicate that belongs to the clay minerals family. The material is made up of 
atomic terraces that naturally emerge when the crystalline material is cleaved. Mica has been used widely as a standard atomically flat substrate in scanning probe microscopy (SPM) to image materials due to its flatness, ease of cleaving, and transparency. In addition, mica has a high electrical resistivity and thermal stability, high dielectric strength $0.1-1$ volt per nanometre $(\mathrm{V} / \mathrm{nm})$, low power loss and large bandgap $10.50 \mathrm{eV}$ [12]. Therefore, mica is a promising insulating material used in high-temperature environments and high electric fields. Moreover, it has a dielectric constant of 6.4-9.3, nearly twice that of traditional $\mathrm{SiO}_{2}$ and h-BN $[13,14]$. Mica has attracted attention due to improved charge homogeneity, lower roughness, and the enhanced carrier mobility of graphene supported on mica substrates. In particular, the morphology of graphene is free of ripples when deposited on atomically flat substrates of mica [9]. Mica substrates not only provide an atomically flat supporting platform for graphene with reduced thermally induced intrinsic ripples, but also open the opportunity of gate dielectric downscaling for mica-based Field Effect Transistors (FETs) because of their high dielectric strength and high dielectric constant [15].

In this thesis, mica is investigated as a candidate substrate for the study of graphene. The properties of annealed graphene on a flat substrate such as mica are not fully understood. It is compared against $\mathrm{SiO}_{2}$ as the leading alternative electrically insulating substrate and the effects of processing the graphene and substrates are considered.

The ability to etch patterns into the mica substrate is also critical, to mark positions and identify locations of samples, and to etch the voids necessary to create the suspended graphene often used in sensing and Micro Electro-Mechanical System (MEMS). However, very little work has been done on lithographic techniques and etching mica, and no recipe exists for electron beam lithography of mica. This will also be investigated in this thesis.

\subsection{Thesis structure}

In chapter two, a brief description of the different techniques is outlined including Raman spectroscopy, scanning electron microscopy (SEM), atomic force microscopy (AFM), and electron beam lithography (EBL). In addition, the principles of operation and general components of each technique are briefly discussed. 
Chapter three includes two sections. Firstly, it describes the graphene structure and properties, including graphene morphology and applications, and addresses and reviews the most common methods for fabricating graphene and producing devices. Secondly, it provides an overview of the mica structure, followed by its properties, and finally its applications.

Chapter four presents the results of the testing method for preparing mica and graphene deposition. Different cleaving methods are tested on mica and its surface after varying the environmental conditions. In addition, the mechanical exfoliation of graphene on mica is tested using different techniques. A comparison of imaging techniques for fewlayer graphene on mica is performed, including optical microscopy, AFM, and SEM. Since graphene is not only deposited on mica substrates but also on $\mathrm{SiO}_{2}$ for comparison purposes, this chapter further reports on thermal oxidation experiments that were carried out to make the $\mathrm{SiO}_{2}$ layer with different oxidation growth thickness. Finally, SEM settings were tested for imaging samples.

Chapter five demonstrates the effect of thermal annealing and substrate morphology on mechanically exfoliated graphene properties on $\mathrm{SiO}_{2}$ and mica substrates with thin and thick graphene sheets. The investigation into the disorder in graphene is characterised using SEM, Raman spectroscopy, and AFM.

Unlike $\mathrm{SiO}_{2}$, mica was found to degrade during the high-temperature vacuum annealing stages usually used to clean samples on $\mathrm{SiO}_{2}$. Chapter six investigates the behaviour of non-patterned bare mica following variable temperature vacuum annealing.

No method has to date been reported for patterning mica substrates using e-beam lithography. This approach is investigated and discussed in detail in chapter seven. At the end of this chapter, work on graphene layers suspended over voids on a mica substrate using e-beam lithography are reported. These are characterised by different techniques, including optical microscopy, AFM and SEM.

Finally, chapter eight contains the conclusion where all results and achievements of this research are summarised. 


\subsection{References}

[1] Alzubi FG, Alkandary A. Substrate induced effect on structural and optical properties of aluminum nanoparticles. Materials Today: Proceedings. 2018;5(5): 13739-13744.

[2] Olsen VS, Bazioti C, Baldissera G, Azarov A, Prytz Ø, Persson C, et al. Effects of substrate and post-deposition annealing on structural and optical properties of ( $\mathrm{ZnO}) 1-\mathrm{x}(\mathrm{GaN}) \mathrm{x}$ films. Physica status solidi (b). 2019;256(6): 1800529.

[3] Hasani E, Kamalian M, Arashti MG, Habashi LB. Effect of substrate properties on nanostructure and optical properties of CdTe thin films. Journal of Electronic Materials. 2019;48(7): 4283-4292.

[4] Geim AK, Novoselov KS. The rise of graphene. Nature Mater. 2007;6(3): 183191.

[5] Alyobi MM, Barnett CJ, Cobley RJ. Effects of thermal annealing on the properties of mechanically exfoliated suspended and on-substrate few-layer graphene. Crystals. 2017;7(11): 349 .

[6] Ando T. Screening effect and impurity scattering in monolayer graphene. Journal of the Physical Society of Japan. 2006;75(7): 074716.

[7] Hwang EH, Adam S, Das Sarma S. Carrier transport in two-dimensional graphene layers. Physical Review Letters. 2007;98(18): 186806.

[8] Gao W, Huang R. Effect of surface roughness on adhesion of graphene membranes. Journal of Physics D: Applied Physics. 2011;44(45): 4.

[9] Lui CH, Liu L, Mak KF, Flynn GW, Heinz TF. Ultraflat graphene. Nature. 2009;462(7271): 339-341.

[10] Goncher SJ, Zhao L, Pasupathy AN, Flynn GW. Substrate level control of the local doping in graphene. Nano Letters. 2013;13(4): 1386-1392.

[11] Yamamoto M, Einstein TL, Fuhrer MS, Cullen WG. Charge inhomogeneity determines oxidative reactivity of graphene on substrates. ACS Nano. 2012;6(9): 8335-8341. 
[12] Lu XF, Majewski LA, Song AM. Electrical characterization of mica as an insulator for organic field-effect transistors. Organic Electronics. 2008;9(4): 473-480.

[13] Dean CR, Young AF, Meric I, Lee C, Wang L, Sorgenfrei S, et al. Boron nitride substrates for high-quality graphene electronics. Nature Nanotechnology. 2010;5(10): 722-726.

[14] Weeks JR Jr. The dielectric constant of mica. Physical Review. 1922;19(4): 319.

[15] Low CG, Zhang Q, Hao Y, Ruoff RS. Graphene field-effect transistors with mica as gate dielectric layers. Small. 2014;10(20): 4213-4218. 


\section{Chapter 2}

\section{Experimental methods}

\subsection{Introduction}

Many different experimental techniques are required to characterise the properties of graphene samples and substrates. Optical microscopy is widely used to monitor graphene sheets under ambient conditions. However, with the wavelength of visible light from 400 to $700 \mathrm{~nm}$, the diffraction limit is a critical factor that limits lowdimensional investigation. As a result, other methods with higher spatial resolution, down to the nanoscale, are required to analyse the graphene samples and substrates in more detail.

This thesis analyses the graphene samples using several techniques. First, SEM is used to assess the morphology of the graphene layers and differentiate between the thickness of the graphene sheets through contrast differences, as well as identify folds in the transferred graphene film. Second, AFM is used for topographic measurements to estimate the number of graphene layers in the samples and to measure substrate roughness. The quality and thickness of the samples are determined using Raman spectroscopy. Raman is also used to investigate the effects of both substrates and thermal annealing on the graphene samples of several thicknesses by the associated defects induced in the graphene. Finally, electron beam lithography is used to pattern the mica substrate.

This chapter briefly discusses the operating theory and basic components of each technique.

\subsection{Scanning electron microscopy}

SEM offers significant advances in the characterisation of the micro-structure. SEM was developed through the 1930s and 1940s, with the first commercial scanning electron microscope produced after many developments of the instrument in 1965 by the Cambridge Instrument Company [1].

SEM is a surface examination device that uses incident electrons and an electron detector to achieve very high resolutions and depths of focus. It fundamentally consists 
of four basic parts, including an electron optical system, specimen chamber, detection system, and display system. First, the source of electrons is produced from the electron gun, being either a field emission gun (FEG), a thermionic gun, or a Schottky gun [2]. The positive charge anode, which is close to the emitter, then accelerates the electrons that form the beam to pass through the column down to the specimen. The beam of electrons passes through the condensing lens along the axis of the column as shown in Figure 2.1. The function of these lenses is to focus the beam into a fine pencil beam with a diameter of up to $10 \mathrm{~nm}$. The fine beam of electrons this produces is scanned across the surface of the specimen. During this process, the electrons of the beam interact with the specimen and give rise to primarily secondary electrons that are detected to form the image.

In addition, backscattered electrons and x-rays are also emitted when the incident electron beam interacts with the specimen. In the normal mode of operation, the secondary electrons are collected through a positively charged Faraday cage detector. SEM has coils that scan the concentrated electron beam in both the $\mathrm{Y}$ and $\mathrm{X}$ directions, as well as to change the scanned area, defined by the ratio of image area width to the beam scan width over the sample.

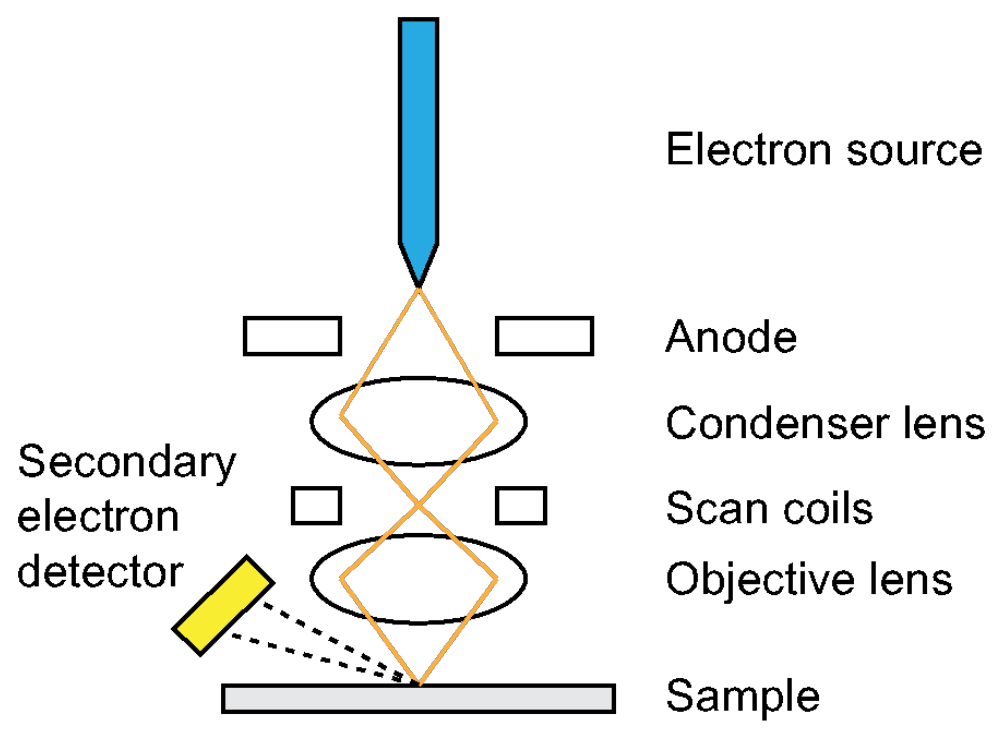

Figure 2.1: Schematic representation of the basic components of an SEM microscopy

In addition to being extensively used to locate graphene sheets, SEM is used to detect folds and discontinuities on different substrates in transferred or manufactured graphene films. According to the literature, SEM contrasts can distinguish between 
graphene sheets with different or altered thicknesses based on the contrast [3-5]. In this study, a Hitachi S-4800 field emission SEM is used. It allows an accelerating voltage from $0.5-30 \mathrm{kV}$ with resolution down to $1 \mathrm{~nm}$. Exact settings are tested and evaluated in Chapter four.

\subsection{Atomic force microscopy (AFM)}

AFM was invented by Binning, Quate, and Gerber in 1986 to overcome the difficulties of measuring non-conductive materials using scanning tunnelling microscopy (STM) [6]. For this reason, it is becoming the chosen tool for scientists interested in physics, chemistry, and biology throughout the world.

AFM operates by scanning a very sharp tip attached to the end of an oscillating cantilever across the sample (see Figure 2.2). The cantilever is usually manufactured from silicon nitride or silicon with a tip radius of curvature on the order of nanometers. When the tip is brought close to a sample surface, forces between the sample and tip cause a deflection of the cantilever according to Hooke's law. Generally, the deflection is measured by using a laser spot reflected from the top surface of the cantilever into an array of photodiodes [7].

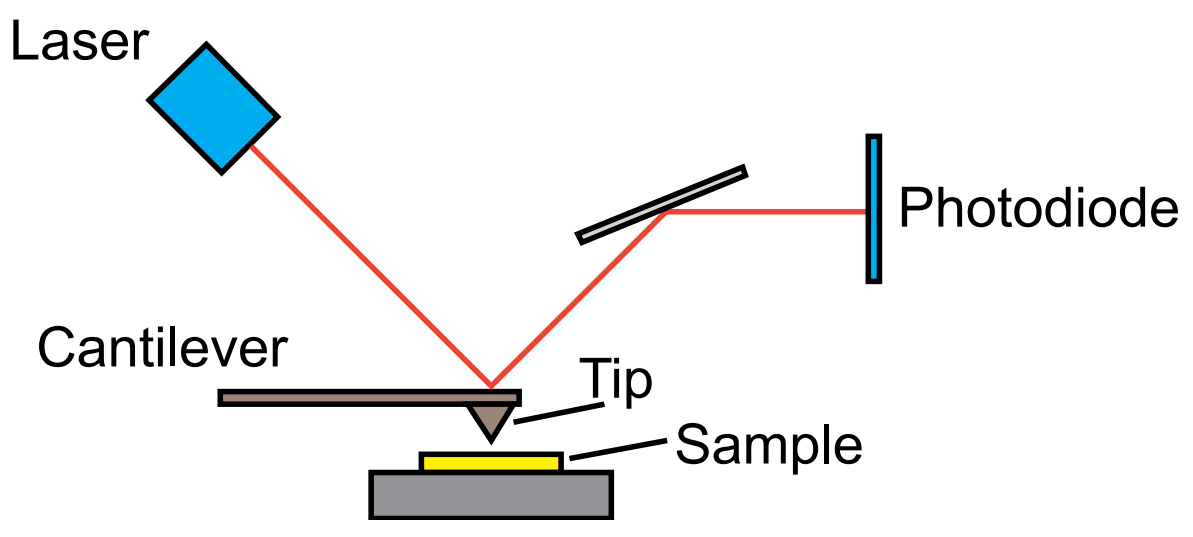

Figure 2.2: Schematic representation of the main principles of AFM.

Different modes can be selected for its operation, depending on the requirements, such as the non-contact mode, contact mode and tapping mode.

\subsubsection{Contact mode}

The tip of AFM stays in contact with a surface during scanning at very low force and its deflection is detected as it scans over the surface. This process provides accurate topographical maps of the sample surface. However, this could lead to damage to the 
soft surface as the cantilever tip is in continuous contact with the sample, which may change both the resulting image and the properties of the material.

\subsubsection{Non-contact mode}

The AFM tip is not in direct contact with the surface of the material and it vibrates close to the surface with a frequency slightly higher than the resonance frequency of the tip. The oscillation amplitude of these vibrations is normally very small, being below $10 \mathrm{~nm}$. When the cantilever retracts from the surface, the attraction of Van der Waals forces decreases the oscillation amplitude and the resonant frequency. Typically, this mode prefers soft material over contact mode because it does not degrade the surface.

\subsubsection{Tapping mode}

The cantilever tip oscillates at a resonant frequency as it scans the surface. When the tip starts to lightly touch the surface, a sensor reverses the cantilever to continue the oscillation. In this way, the tip does not continuously touch the surface to avoid any damage. However, because the cantilever is not continuously interacting with the surface, a method must be established to measure the differences in surface height because of changes in the amplitude of oscillation of the cantilever. Furthermore, when it encounters a strike on the surface, the amplitude of oscillation is decreased. Conversely, when the sample height reduces, the cantilever amplitude increases. By recording these changes, an accurate topographical map could be generated without causing any damage to the surface of the material.

AFM has extensively been used to determine graphene film thicknesses through topographic measurements, as well as to investigate the mechanical properties such as intrinsic strength, spring constant, and Young's modulus [8,9].

In this work, a Nanosurf Naio AFM under ambient conditions was used to demonstrate the morphology of graphene on $\mathrm{SiO}_{2} / \mathrm{Si}$ and mica to indicate the graphene number layer. Subsequently, Gwyddion software was used to analyse the AFM images of graphene and obtain the average profile line. AFM contact mode was used to measure the thickness of graphene to determine the number of layers in chapter five while tapping mode was used in Chapters six and seven. 


\subsection{Raman spectroscopy}

The phenomenon of inelastic scattering of light by matter was observed experimentally for the first time by Raman in 1928 [10]. In 1930, he won the Nobel Prize in physics for his work on the scattering of light. It relies on the interaction of light with the chemical bonds in a material. Raman spectroscopy is known to provide detailed information about molecular interactions, crystallinity, and chemical structure.

The mechanism for Raman scattering depends on the change in rotation or vibration quantum states of illuminated molecules. For example, Rayleigh scattering occurs when light focuses on a sample and most of the scattering takes place elastically, without the loss of energy or change in frequency (see Figure 2.3). However, Raman scattering is caused by the inelastic scattering from incident photons where energy is transferred to, or received from, the material due to changes in the vibrational or rotational modes of molecules. This produces changed energy, and consequently, the frequency of the scattered light. It was observed that if the incident photon gives up energy to the atom, it is scattered with a red-shifted frequency called the Stokes shift (Figure 2.3). In addition, if the molecule is already in an exited energy state, where energy was given to the scattered photon, then the production has a blue-shifted frequency, called an anti-stokes shift (Figure 2.3).

a)

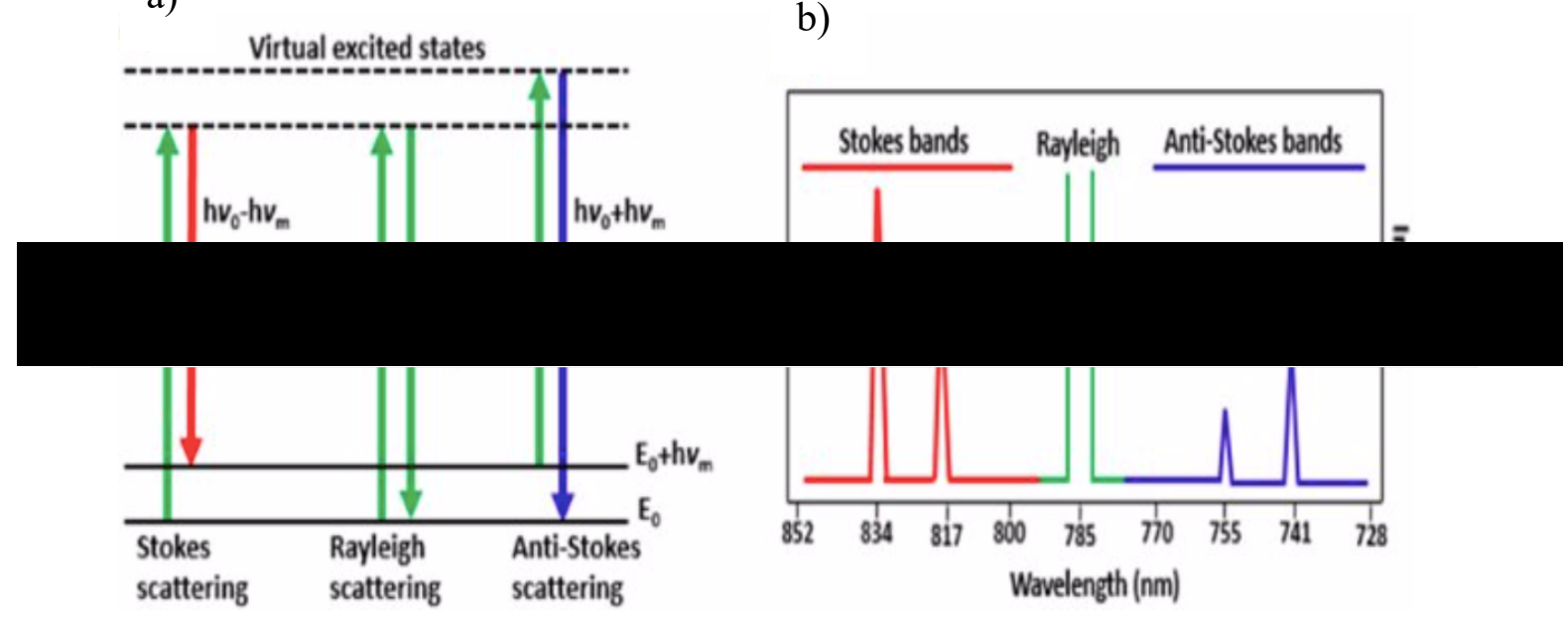

Figure 2.3: Schematic representation of (a) Rayleigh and Raman scattering, and (b) the resulting Raman spectrum [11]. 
Figure 2.4 schematically represents Raman spectroscopy (Renishaw) that contains three major parts, being a source, an analyser, and a microscope [12]. The source of the photon beam is a laser that is guided onto the sample by using microscope objectives, which also redirect the back-scattered beam to the spectrum analyser. This is used to measure the energy spectrum of the scattered beam.

First, the photon beam (blue) passes through density filters that reduce the intensity of the received photon beam. Following this, it passes through a photon beam expander, which is a pair of lenses that have the responsibility of collimation of the photon beam. The expansion of the beam leads to a small laser spot focused on the material. Typically, a pinhole is located between the lenses of the expander which contributes to improving the image of the photon beam by converting a line laser into a spot laser. The photon beam is aligned using motorised and other mirrors that direct the laser beam onto the sample through the objective lens. The photon beam interacts with the atoms in the sample as mentioned above to generate the elastic and inelastic scattered photons. The objective lens used to focus the photon beam is also responsible for collecting the Raman and Rayleigh scattered signals.

The scattered beam path (red) passes initially through directional optics, then through the edge filter that allows the scattered beam of Stokes and/or the Anti-Stokes Raman to transmit. It does this while blocking the Rayleigh scattered beam to ensure that the detector will not be damaged as the highest proportion of the photon beam results from Rayleigh scattering. After the edge filter, the lens focuses the Raman beam into the beam splitter together with another lens that is used to expand the beam.

Both the edge filter and beam splitter can be accomplished with a single optical component named a dichroic mirror. The photon beam is collimated on a diffraction grating by reflection from the mirror and is thereafter refocused onto a charge-coupled device (CCD) sensor in the focal plane of the final lens. This sensor is used to convert the incoming beam into an electrical signal, as well as collecting counts between each reading process as requested by the software [13]. 


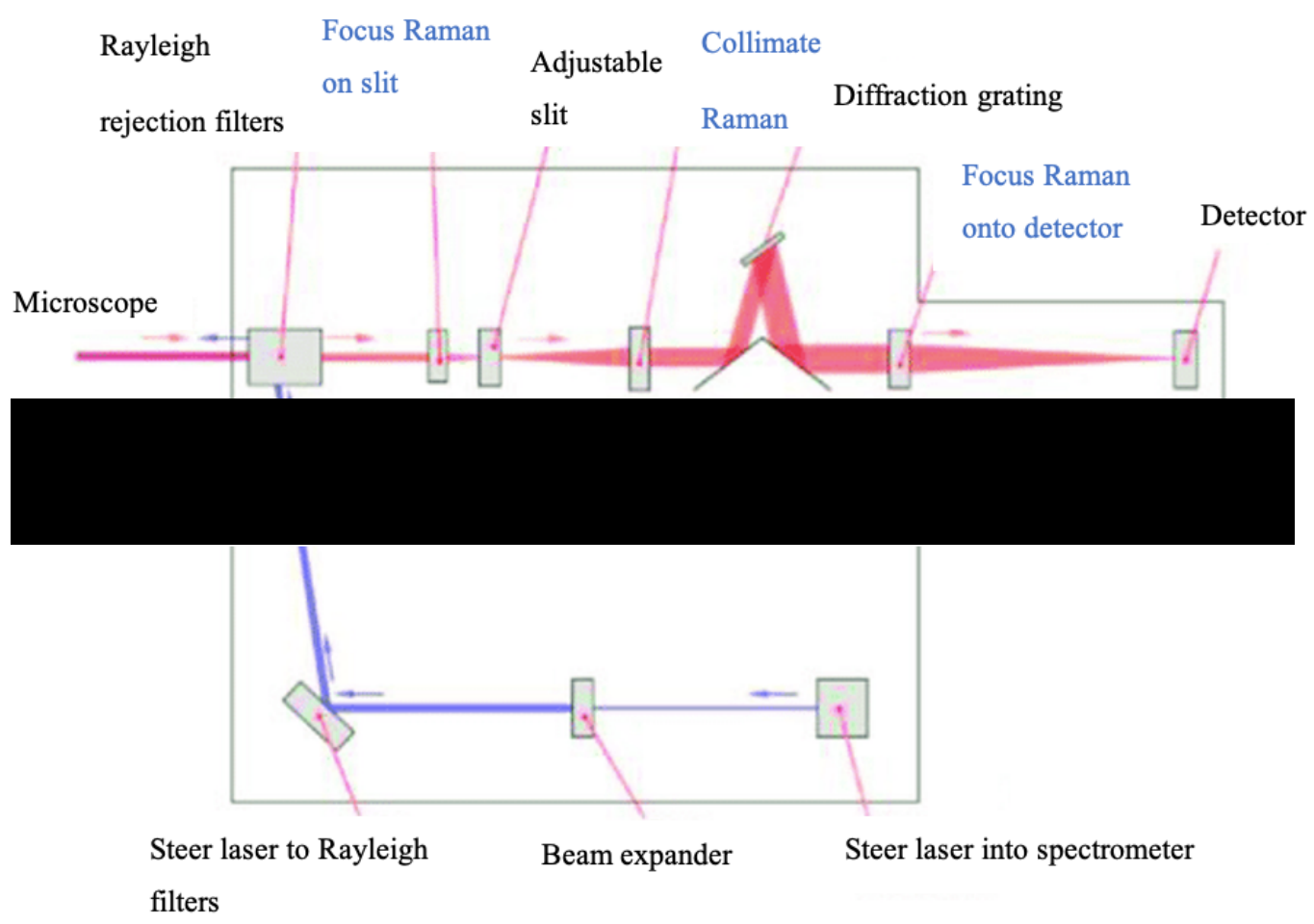

Figure 2.4: Schematic representation of the Raman spectroscopy components [12].

\subsubsection{Raman spectra for graphene}

Recently, many researchers have focused on Raman scattering studies for the investigation of several types of graphene samples. According to the literature, the number of layers of graphene was studied, with a special focus on the possibility of using the Raman spectrometer to distinguish a single layer from multiple layers of graphene stacked in the Bernal (AB) configuration. This confirms the importance of Raman spectra in studying and characterising graphitic materials- The Raman spectroscopy technique used for identifying the number of graphene sheets was established only for graphene with $\mathrm{AB}$ Bernal stacking. AB Bernal stacking features are layers of graphene where half of their atoms lie directly over the hexagon centre in the lower graphene sheet, and half of the atoms lie over an atom. In addition, Bernal stacked bilayer graphene displays much interest for both functional electronic and photonic devices as a result of the feasibility to continuously tune its band gap with a vertical electrical field. This type of sample is obtained from highly oriented pyrolytic graphite (HOPG) created by mechanical exfoliation [14].

The Raman spectra generated by $\mathrm{sp}^{2}$ nanocarbons are very rich and consist of many peaks induced by single- and multiple-resonance effects. The Raman features can all 
be related to phonons in graphene, at the $\Gamma$ point, inside the Brillouin zone and near the Brillouin zone boundary [15]. However, of all the peaks to appear in the Raman spectra of graphene, three principle bands convey most of the required structural and chemical information, being the G, D and 2D bands [16, 17]. Although some sources refer to the $2 \mathrm{D}$ band as $\mathrm{G}^{\prime}$ in the figures, all experimental work in this paper will identify the peak as $2 \mathrm{D}$.

\subsubsection{The G-band properties}

The properties of the G-band have been summarised below [15]:

1. The G-band is the Raman signature for $\mathrm{sp}^{2}$ carbons and is shown as a peak (or a multi-peak feature) at about $1585 \mathrm{~cm}^{-1}$ for all $\mathrm{sp}^{2}$ carbon kinds (Figure 2.5).

2. Uniaxial stretching of graphene splits the $G$ peak into both $\mathrm{G}^{-}$and $\mathrm{G}^{+}$, which are associated with the atomic motion along, and perpendicular to, the stretching directions, respectively. Redshifts, both $\omega^{+}$and $\omega_{G}^{-}$, occur when the stretching increases (Figure 2.6).

3. Temperature $(T)$ : usually, increasing $T$ redshifts $\omega_{G}$. Different effects occur, such as changes in phonon-phonon coupling, the electron-phonon renormalisation, and $\omega_{\mathrm{G}}$ shifts due to thermal expansion caused by volume changes. The $\omega_{\mathrm{G}}$ shift with temperature has been used to determine the thermal conductivity of graphene. 


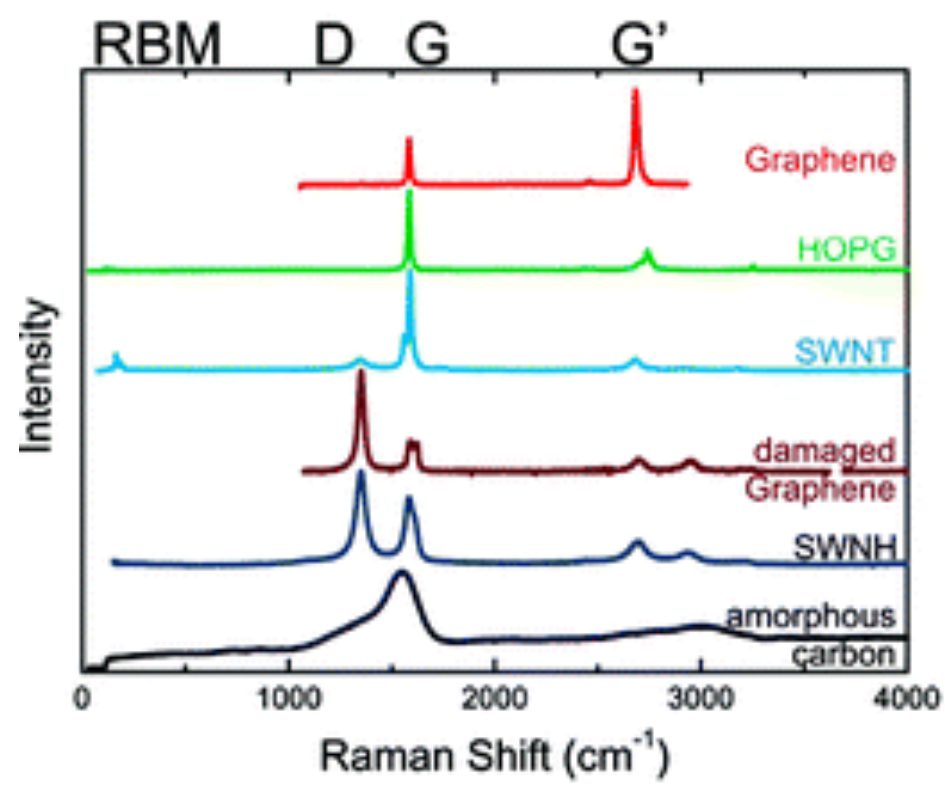

Figure 2.5: Raman signatures from several types of nanocarbon material. From bottom to top : amorphous carbon, single-wall carbon nanohorns (SWNH), damaged graphene, a single-wall carbon nanotube (SWNT), highly oriented pyrolytic graphite (HOPG) and crystalline monolayer graphene. The term $G^{\prime}$ band in this figure is referred to as the 2D band in this thesis [18].

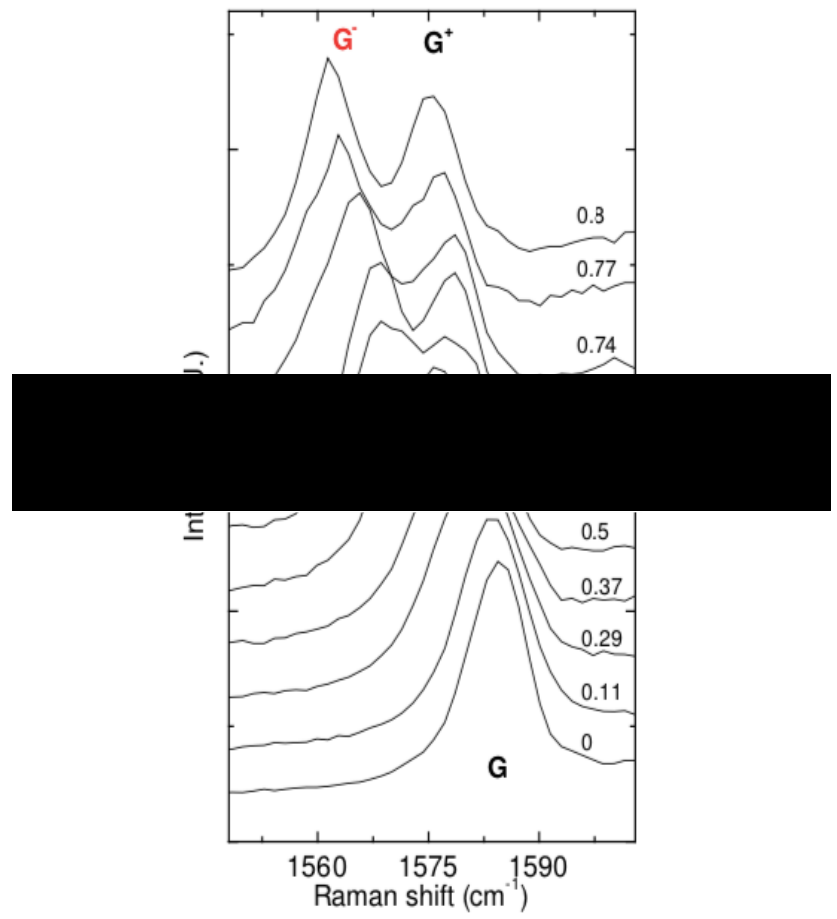

Figure 2.6: The G-band split into $\mathrm{G}^{-}$and $\mathrm{G}^{+}$. As the strain increases, the splitting increases [19]. 


\subsubsection{The D-band properties}

The properties of the D-band have been summarised below [15]:

1. The D-band is produced by the disordered structure of carbon materials. The D-band occurs as a peak in the range $1250-1400 \mathrm{~cm}^{-1}$ (Figure 2.5).

2. The D-band frequency: $\omega_{\mathrm{D}}$ has a peak at $\sim 1350 \mathrm{~cm}-1$ for $E_{\text {laser }}=2.41 \mathrm{eV}$. However, its frequency changes with the changing $E_{\text {laser. }}$

3. The D-band intensity can be used to quantify disorder. Because of the difficulty in establishing an absolute intensity measurement in Raman spectroscopy, the normalised intensity $\mathrm{I}_{\mathrm{D}} / \mathrm{I}_{\mathrm{G}}$ ratio is widely used to measure the amount of disorder. Although this ratio depends on the amount of disorder, it is independent of the excitation laser energy.

\subsubsection{The 2D-band properties}

The properties of the 2D-band have been summarised below:

1. The D band is produced by the breathing modes of sp atoms and is activated by doubly resonant disorder. Consequently, it is a significant indicator of the sample quality. It disappears in defect-free graphene and graphite samples as mentioned above. However, the 2D peak is the second order of the $\mathrm{D}$ peak and is always present even when $\mathrm{D}$ band disappears, as no defects are needed to activate the second-order phonons. In some studies, the 2D band is also called the $\mathrm{G}$ band, due to it is the second-most distinguished band of graphene after the $\mathrm{G}$ band. The change of the positions and intensities of these bands is used to measure graphene properties, including thickness, structural deformation and doping. The Raman 2D band is considered to be very sensitive to the number layers of graphene. As well, the 2D band of single-layer graphene is very sharp and symmetric. The $2 \mathrm{D}$ band obviously becomes much broader for multiple and few layers as a consequence of the change in the graphene electronic structure [16].

2. The $2 \mathrm{D}$ band results from a second-order two phonon process observed in nanocarbon materials as a peak, such as single-layer graphene, or a multi-peak feature like multilayer highly oriented pyrolytic graphite in the range of 2500 $2800 \mathrm{~cm}^{-1}[15]$. 
3. The sensitivity of $\omega_{\mathrm{G}}{ }^{\prime}$ to the structure of $\mathrm{sp}^{2}$ makes this band a significant tool for quantifying the number of graphene layers. One layer of graphene (1-LG) shows a single Lorentzian peak in the $\mathrm{G}^{\prime}$-band. Figure 2.7 illustrates how sharp and symmetrical the shape of the monolayer graphene is, as well as how it broadens as the layers increase. In addition, the quantitative guide for the number of layers is the full width at half maximum (FWHM) for the 2D band. As shown in Figure 2.7b, the typical FWHM of 2D peaks of numerous fewlayer graphene (FLGs) are plotted. There were consistent, substantial, and different ranges for single-, bi-, tri-, four-, and five-layer graphene at $27.5 \pm$ $3.8 \mathrm{~cm} 1,51.7 \pm 1.7 \mathrm{~cm}^{-1}, 56.2 \pm 1.6 \mathrm{~cm}^{-1}, 63.1 \pm 1.6 \mathrm{~cm}^{-1}$, and $66.1 \pm 1.4 \mathrm{~cm}^{-1}$, respectively where flake counter is the sample number. Also, there is no evidence to see any overlap for different thicknesses of FLG, and the data can be a straightforward standard in order to determining the layer number of graphene [20]. Another method to demonstrate the number of layers is the ratio of the intensity of the $2 \mathrm{D}$ band to the intensity of the $\mathrm{G}$ band per Figure 2.8. For instance, the intensity of the $\mathrm{G}^{\prime}$-band is twice as large as the G-band in 1LG. This ratio reduces with increasing graphene thickness [15].

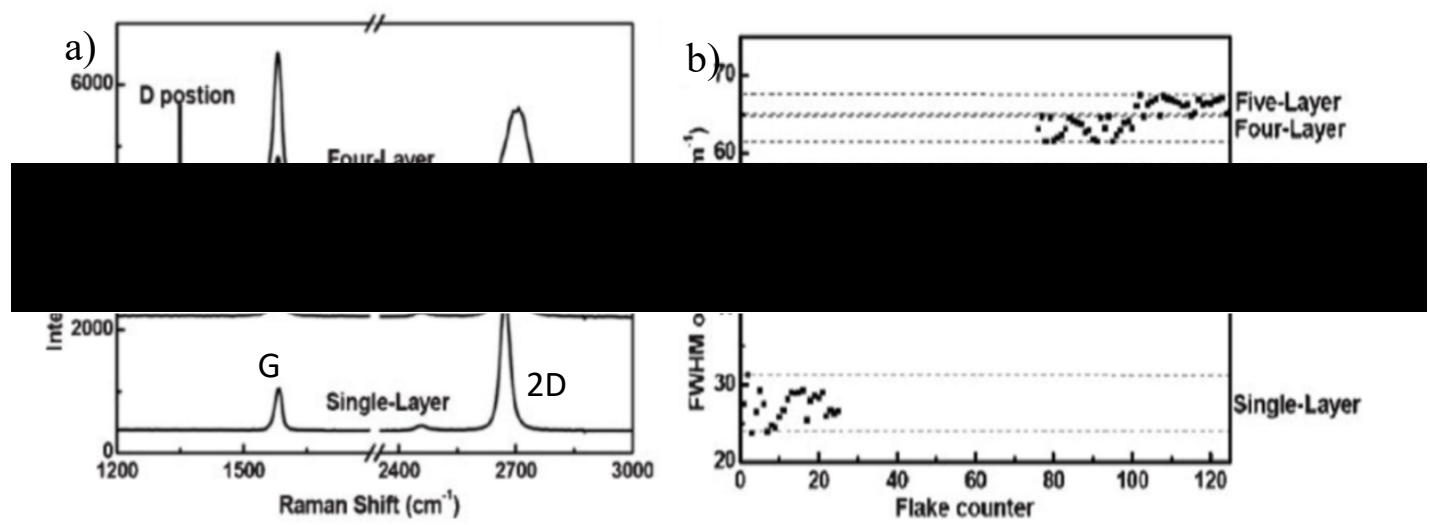

Figure 2.7: Raman spectra to determine the number of layers in graphene based on a) Shape of $2 \mathrm{D}$ and intensity $\mathrm{I}_{2 \mathrm{D}} / \mathrm{I}_{\mathrm{G}}$, and $\mathrm{b}$ ) FWHM of the 2D band [20]. 

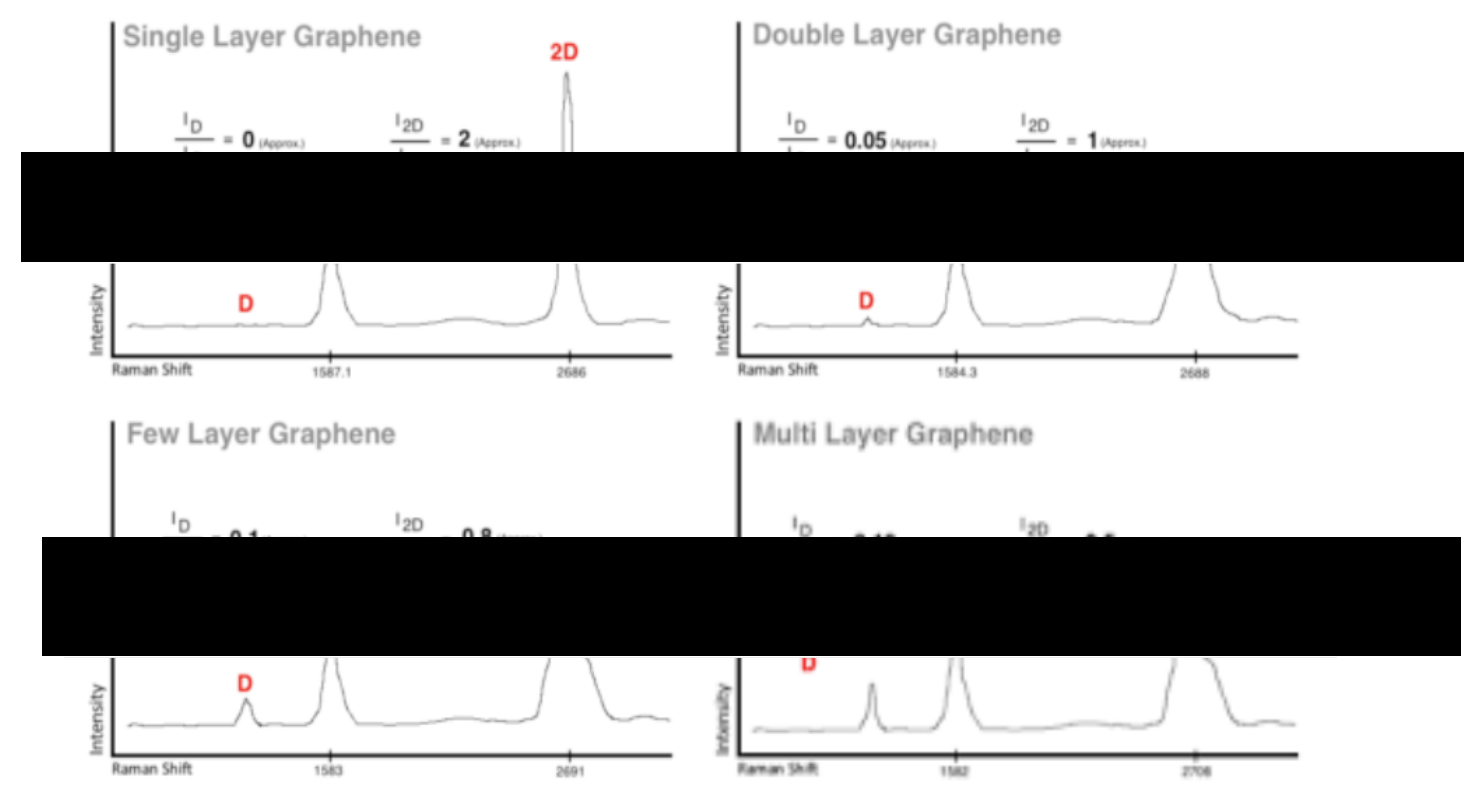

Figure 2.8: Raman spectra to determine the number of layers in graphene based on an intensity ratio [21].

\subsection{Electron beam lithography (EBL)}

It is well-known that the origins of lithography date back to the seventeenth century in ink printing applications. Currently, there are a wide variety of applications and techniques thereof, with the concept remaining valid. Lithography in device fabrication includes the processes that are used to transfer a pattern from one form of media to another. Common types of lithography include both photolithography and electron beam lithography (e-beam). Photolithography uses light as the transfer mechanism and is extensively applied in technological processes. Its high yield makes it ideal for the semiconductor industry, particularly when applied to silicon technology in integrated circuit manufacturing. However, photolithography is not suitable to fabricate very small structures of $\leq 100 \mathrm{~nm}$ because of light diffraction [22].

Consequently, electron beam lithography has become a common choice for device fabrication, with advantages including very high resolution and versatile pattern shaping [22]. EBL arose in the late 1960s by modifying the design of SEM. The essential difference between them is that the beam in SEM is raster-scanned over the sample to produce an image, whereas the beam in EBL is directed according to the designed pattern created by the software generator. The limit of resolution of an optical 
system is dependent on both the numerical aperture and the wavelength of light (according to Law of Ernst Abbé). This law holds in addition for electrons, of which the speed determines their wavelength, thus the higher the speed of the electrons, the smaller the wavelength and the better the resolution [23].

In summary, there are several common attributes of the technology, such as 1) having a very high spatial resolution, 2) being a flexible technique that can be used with a variety of materials, 3) being one or several orders of magnitude slower in comparison with optical lithography, and 4) being complicated and expensive due to it requiring more frequent maintenance.

\subsubsection{EBL process}

This EBL process involves three steps, being the exposure of the sensitive material, transmission of patterns, and the development of the resist (Figure 2.9).

A thin layer of resist, based on an analogy with photoresist and using the term ' $\mathrm{e}$ resist', is used to coat the substrate that is chemically varied under exposure to the electron beam. This allows the exposed and non-exposed areas to be dissolved in a suitable solvent, being either positive or negative lithography, through an equally important process called 'development' (using the same analogy of the development of photographic films) [24]. After development and exposure, the resist layer at the top of the sample can be used as a template or mask to transfer the pattern into a more advantageous medium. The process of development and the resist must be closely coupled and associated with the functional dependencies of the electron beam tool [24].

In addition, two major pattern transfer techniques can be utilised. The first uses etching material underneath the voids in the resist layer. The second involves depositing or layering a material, generally metal, over the sample and removing the residual resist to lift the deposited material on top. This leaves the deposited material only in the areas where there is no resist. Figure 2.9 shows the e-beam process for a negative resist, where what is exposed remains after developing. This is the process that is subsequently developed in this paper to pattern mica. A similar process is involved with the more common positive e-resist, except that what is exposed to the e-beam remains, and the reverse pattern is obtained. 


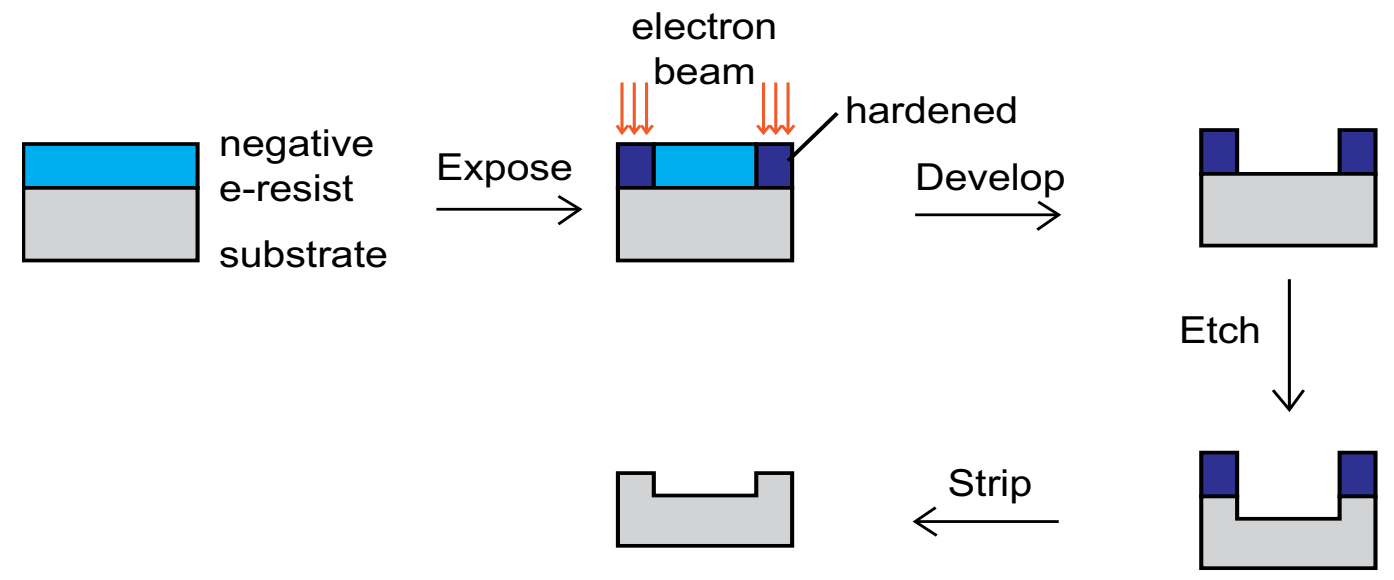

Figure 2.9: A schematic illustration of the EBL process for a negative e-resist.

\subsection{Summary}

This chapter presented the principle experimental techniques used in this study. Specific equipment settings have been provided here to develop them further in the study. The next chapter examines the materials used, after which the results chapters utilise the experimental techniques discussed herein. 


\subsection{References}

[1] Stokes D. Principles and Practice of Variable Pressure/Environmental Scanning Electron Microscopy (VP-ESEM). New York: John Wiley \& Sons; 2008.

[2] Bogner A, Jouneau PH, Thollet G, Basset D, Gauthier C. A history of scanning electron microscopy developments: Towards "wet-STEM" imaging. Micron. 2007 Jun 1;38(4):390-401.

[3] Hiura H, Miyazaki H, Tsukagoshi K. Determination of the number of graphene layers: discrete distribution of the secondary electron intensity stemming from individual graphene layers. Applied Physics Express. 2010;3(9): 095101.

[4] Xie J, Spallas JP. Different Contrast Mechanisms in SEM Imaging of Graphene. Agilent Technologies. Application Note: 5991-0782EN, 2012.

[5] Kochat V, Nath Pal A, Sneha ES, Sampathkumar A, Gairola A, Shivashankar SA. High contrast imaging and thickness determination of graphene with in-column secondary electron microscopy. Journal of Applied Physics. 2011;110(1): 014315.

[6] Binnig G, Quate CF, Gerber C. Atomic force microscope. Physical Review Letters. 1986;56(9): 930.

[7] Wang H, Chu PK. Surface characterization of biomaterials. In: Bandyopadhyay A, Bose S. (eds.) Characterization of Biomaterials. USA: Elsevier; 2013. p.105174.

[8] Annamalai M, Mathew S, Jamali M, Zhan D, Palaniapan M. Elastic and nonlinear response of nanomechanical graphene devices. Journal of Micromechanics and Microengineering. 2012;22(10): 105024.

[9] Frank IW, Tanenbaum DM, van der Zande AM, McEuen PL. Mechanical properties of suspended graphene sheets. Journal of Vacuum Science \& Technology B: Microelectronics and Nanometer Structures Processing, Measurement, and Phenomena. 2007;25(6): 2558-2561.

[10] Raman CV. A change of wave-length in light scattering. Nature. 1928;121(3051): 619. 
[11] Lohumi S, Kim MS, Qin J, Cho BK. Raman imaging from microscopy to macroscopy: Quality and safety control of biological materials. TrAC Trends in Analytical Chemistry. 2017;93: 183-198. Available from: doi.org/10.1016/j.trac.2017.06.002. Permission no 4864261274501

[12] Casimir D, Alghamdi H, Ahmed IY, Garcia-Sanchez R, Misra P. Raman spectroscopy of graphene, graphite and graphene nanoplatelets. In: Wongchoosuk C, Seekaew Y. (eds.) 2D Materials. IntechOpen; 2019. Available from: doi:10.5772/intechopen.84527. Attribution 3.0 Unported (CC BY 3.0) https://creativecommons.org/licenses/by/3.0/

[13] Alyobi MM, Local probe electrostatic manipulation of graphene: a study of the electronic and mechanical properties of rippled graphene sheets. Doctoral dissertation, Swansea University, 2017.

[14] Bîru EI, Iovu H. Graphene nanocomposites studied by Raman spectroscopy. Raman Spectroscopy. 2018 Jan 26;179.

[15] Jorio A, Dresselhaus MS, Saito R, Dresselhaus G. Raman Spectroscopy in Graphene Related Systems. New York: John Wiley \& Sons; 2011.

[16] Ferrari AC, Meyer JC, Scardaci V, Casiraghi C, Lazzeri M, Mauri F, et al. Raman spectrum of graphene and graphene layers. Physical Review Letters. 2006;97(18): 187401.

[17] Casiraghi C, Pisana S, Novoselov KS, Geim AK, Ferrari AC. Raman fingerprint of charged impurities in graphene. Applied Physics Letters. 2007;91(23): 233108.

[18] Dresselhaus MS, Jorio A, Hofmann M, Dresselhaus G, Saito R. Perspectives on carbon nanotubes and graphene Raman spectroscopy. Nano Letters. 2010;10(3): 751-758. Figure reprinted with permission from this source, Copyright (2020) by American Chemical Society.

[19] Mohiuddin TMG, Lombardo A, Nair RR, Bonetti, A, Savini G, Jalil R, et al. Uniaxial strain in graphene by Raman spectroscopy: G peak splitting, Grüneisen parameters, and sample orientation. Physical Review B. 2009;79(20): 205433. Permission no. RNP/20/JUL/027979. 
[20] Hao Y, Wang Y, Wang L, Ni Z, Wang Z, Wang R, et al. Probing layer number and stacking order of few-layer graphene by Raman spectroscopy. Small. 2010;6(2): 195-200. Permission no. 4814310440672.

[21] InstaNANO. Image caption [Internet image]. Graphene Number of Layers Calculator. Available from: https://www.instanano.com/2017/03/RamanCharacterization-Graphene.html [Accessed 20th July 2020]. Copyright (2020) by InstaNANO.'

[22] Tseng AA, Chen K, Chen CD, Ma KJ. Electron beam lithography in nanoscale fabrication: recent development. IEEE Transactions on Electronics Packaging Manufacturing. 2003;26(2): 141-149.

[23]. Schellenberg F. A little light magic [optical lithography]. IEEE Spectrum. 2003 Sep 8;40(9):34-9.

[24] Tennant DM, Bleier AR. Electron beam lithography of nanostructures. In: Wiederrecht G. (ed.) Handbook of Nanofabrication. Amsterdam: Elsevier; 2010. p.121-148. 


\section{Chapter 3}

\section{Materials}

\subsection{A brief history of graphene}

Although graphene research intensified after the year 2000, it is well-known that experimental studies of graphene date back to the early 1960s when the conductivity of thin graphite was found to be surprisingly higher than that of the original graphite. Ultrathin graphite layers were observed under several conditions, but due to technical complications, the research of graphene has evolved slowly. It was a complicated task both theoretically and experimentally to demonstrate a material that a conductor, lighter and less expensive [1]. Although graphene research began in the 1960s, known then as graphite layers, it has grown dramatically since 2004 when Andre Geim and Konstantin Novoselov from Manchester University produced monolayer and fewlayer graphene for the first time. They isolated it from graphite as a starting material using Scotch tape, then deposited it on a $\mathrm{SiO}_{2} / \mathrm{Si}$ substrate [2]. This method is advantageous because it is simple and effective in producing a high-quality graphene sheets. In 2005, the Manchester University group showed the two-dimensional massless Dirac fermions in graphene unambiguously in a consecutive paper in the Nature Journal [3].

The experiments not only confirmed many predictions of graphene's usual electronic properties but also discovered various novel characteristics of the material. In 2010, the Nobel Prize in physics was given to A.K. Geim and K.S. Novoselov for their pioneering contributions in graphene research. The award provided strong evidence of the impact of graphene on technology and science.

\subsection{Graphene properties: Structural properties}

Graphene is a two-dimensional material made up of carbon atoms that are arranged in a honeycomb structure. As shown in figure 3.1 a) graphene crystal contains two carbon atoms $\mathrm{A}$ and $\mathrm{B}$ [4]. Their lattice vectors are written as:

$$
\vec{a}_{1}=\frac{a}{2}(1, \sqrt{3}) \text { and } \vec{a}_{2}=\frac{a}{2}(1,-\sqrt{3})
$$


where $\mathrm{a}=\sqrt{ } 3 \mathrm{a}_{0} \approx \sqrt{ } 3 \times 1.421=2.46 \AA$ is the lattice constant, which is the distance between unit cells. The position vector of atom $B_{1},(l=1,2,3)$ relative to the atom $A_{i}$ is demonstrated as $\delta_{1}$, while the three nearest neighbour vectors in real space are given by

$$
\begin{gathered}
\vec{\delta}_{1}=\left(0, \frac{a}{\sqrt{3}}\right), \vec{\delta}_{2}=\left(\frac{a}{2},-\frac{a}{2 \sqrt{3}}\right) \text { and } \\
\vec{\delta}_{3}=\left(-\frac{a}{2},-\frac{a}{2 \sqrt{3}}\right)
\end{gathered}
$$

It is noted that $\left|\delta_{1}\right|=\left|\delta_{2}\right|=\left|\delta_{3}\right|=\mathrm{a} / \sqrt{3}$ is the spacing between two nearest-neighbouring carbon atoms. Figure 3.1(b) shows the reciprocal lattice of single layer, where the crosses are reciprocal lattice points, and the shaded hexagon is the first Brillouin zone. The reciprocal lattice vectors $b_{1}$ and $b_{2}$ address the conditions

$$
\begin{gathered}
\left\{\begin{array}{l}
\vec{a}_{1} \vec{b}_{1}=\vec{a}_{2} \vec{b}_{2}=2 \pi \\
\vec{a}_{1} \vec{b}_{2}=\vec{a}_{2} \vec{b}_{1}=0
\end{array}\right. \\
\vec{b}_{1}=\left(\frac{2 \pi}{a}, \frac{2 \pi}{\sqrt{3} a}\right) \text { and } \vec{b}_{2}=\left(\frac{2 \pi}{a},-\frac{2 \pi}{\sqrt{3} a}\right)
\end{gathered}
$$

Figure $3.1 \mathrm{c}$ ) shows the carbon atom nucleus has six electrons $1 \mathrm{~s}^{2} 2 \mathrm{~s}^{2} 2 \mathrm{p}_{\mathrm{x}}{ }^{1} 2 \mathrm{p}_{\mathrm{y}}{ }^{1} 2 \mathrm{p}_{\mathrm{z}}{ }^{0}$. In 1s states, there are two electrons in the core shell, which do not participate in atomic bonding and are strongly bound to the nucleus. The four electrons refer to the valence shell that forms three hybridisation types, which are $\mathrm{sp}, \mathrm{sp}^{2}$ and $\mathrm{sp}^{3}$. The difference in energy between the $2 \mathrm{~s}$ and $2 \mathrm{p}$ orbitals was found to be less than the energy gain through $\mathrm{C}-\mathrm{C}$ binding. For this reason, when carbon atoms bind, their $2 \mathrm{~s}$ and $2 \mathrm{p}$ orbitals can be combined with one another in $\operatorname{sp}^{n}(n=1,2,3)$ hybridised orbitals. For monolayer graphene, carbon atoms share $\mathrm{sp}^{2}$ electrons with their three neighbouring carbon atoms (Figure 3.1). At this point, each carbon atom has three close neighbours, forming the hexagonal planar network of graphene [4], whereas, in the structure of a diamond, the orbitals for one $2 \mathrm{~s}$ and three $2 \mathrm{p}$ electrons combine, making four $\mathrm{sp}^{3}$ orbitals, binding each carbon atom to four carbon neighbours at the vertices of a regular tetrahedron. 

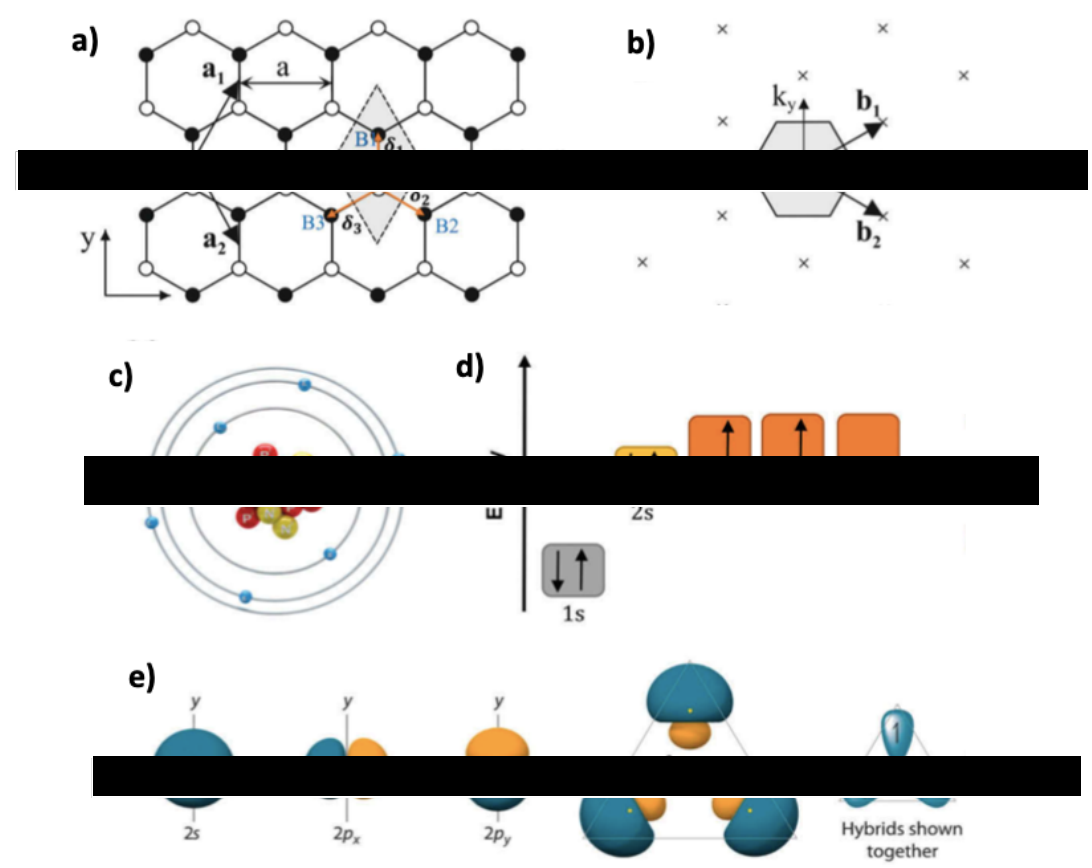

Figure 3.1: (a) Honeycomb lattice of monolayer graphene, where white (black) circles indicate carbon atoms on A (B) sites, and (b) the reciprocal lattice of monolayer graphene, where the shaded hexagon is the corresponding Brillouin zone (c) Carbon atom structure. (d) Energy levels of outer electrons in carbon atoms. (e) The production of $\mathrm{sp}^{2}$ hybrids [4].

There are many allotropes of carbon that form a variety of different nanostructures. Figure 3.2 illustrates that graphite's three dimensions are made up of multiple layers of 2D graphene linked by weak van der Waals interactions. Rolling a single layer of graphene into a cylinder produces a single-walled carbon nanotube (SWCNT), while several rolled cylinders create a multi-wall carbon nanotube (MWCNT), both of which are 1D carbon. However, rolling graphene into a ball results in a fullerene (Buckyball) which approaches a 0D system [5]. 


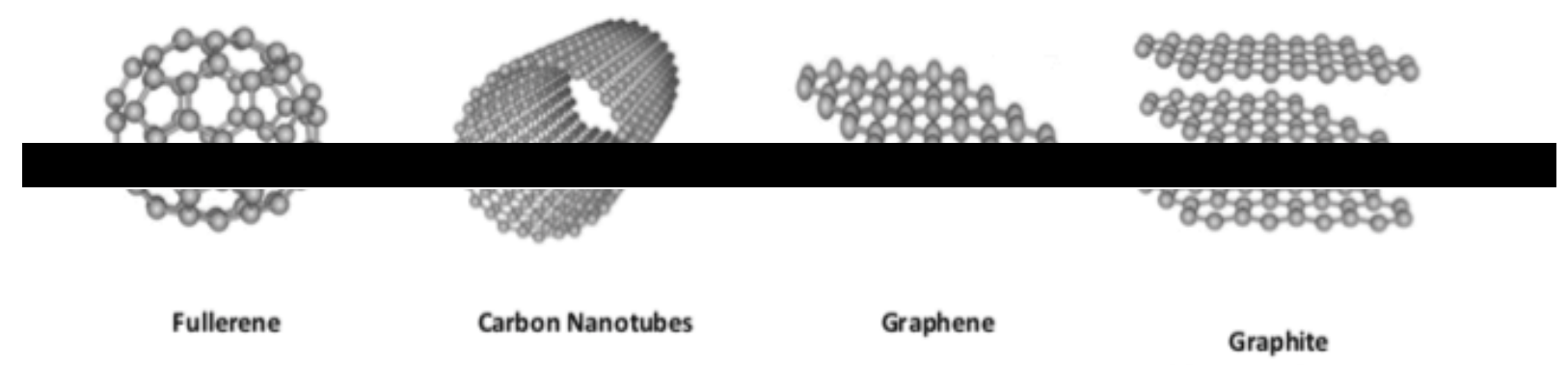

Figure 3.2: $\mathrm{Sp}^{2}$ carbon materials [6].

\subsubsection{Optical properties}

A single graphene sheet can only absorb $2.3 \%$ of incident light, but the absorption increases linearly with the increase in the number of graphene layers [7]. Individual graphene layers can absorb light from $300 \mathrm{~nm}$ (near-ultraviolet, NUV) to $2500 \mathrm{~nm}$ (near-infrared, NIR) [8]. Graphene's thin transparent sheet cannot be detected by the human eye alone; it requires a substrate such as $\mathrm{SiO}_{2}$ that can be characterised by the optical image contrast. With increasing graphene thickness, such contrast increases [9].

The unique properties of graphene lead to many controllable photonic devices. These include solid-state-mode-locked lasers as graphene saturable absorbers that are much simpler and less expensive to integrate into the laser system compared to semiconductor saturable absorbers [10].

\subsubsection{Electronic properties}

Unlike bulk semiconductors, where there is a gap between the conduction band minimum and the valence band maximum, graphene is a zero-band semiconductor. The Dirac cones meet at six Dirac points in momentum space, where electrons effectively lose their mass and become quasi-particles. This is because each atom contains three valence electrons, $s, p_{x}$, and $p_{y}$, that share the $\sigma$-bond with its closest respective neighbour, whereas the fourth valence electron, $\mathrm{p}_{z}$, forms the delocalised $\pi$ bond as shown in Figure 3.3. These $\pi$-bonds hybridise to produce the $\pi$-band and $\pi^{*}$ bands that are responsible for most of the promising electronic properties in graphene [4]. 


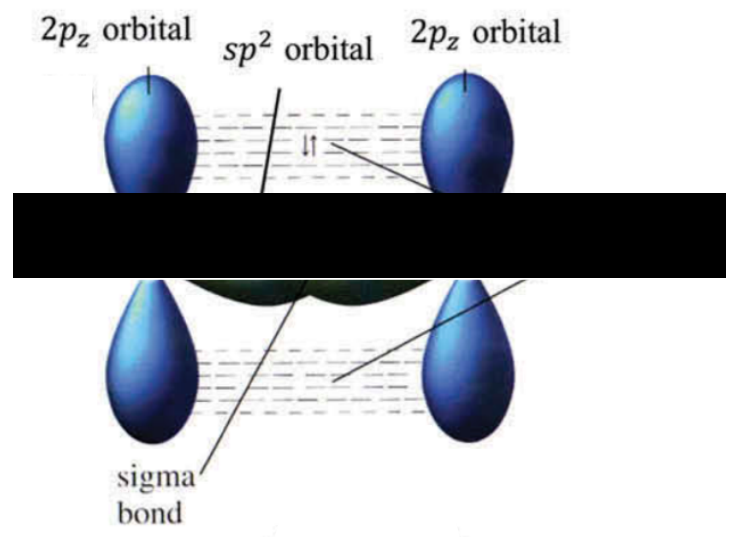

Figure 3.3: Sigma and pi bonds formed by $\mathrm{sp}^{2}$ hybridisation .[4]

Graphene has an energy structure known as the Dirac cone made from two cones. The small point between the cones is defined as the Dirac point. They are $\mathrm{K}$ and $\mathrm{K}$ ' when valence and conduction bands are degenerated to produce graphene zero band gap semiconductors as shown in Figure 3.4. However, the nature of graphene can mysteriously remain as a semiconductor or a semimetal, owing to the gapless features thereof.

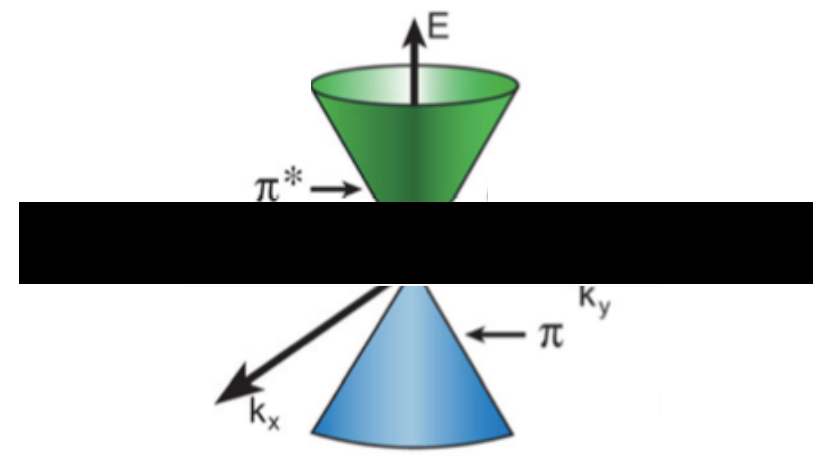

Figure 3.4: band structure of pristine graphene with two cones at a Dirac point [11]

It has been reported that graphene has a very high charge in electron and hole mobility, being approximately $230,000 \mathrm{~cm}^{2} \cdot \mathrm{V}^{-1} \cdot \mathrm{S}^{-1}[12]$. However, the defect in the graphene device causes charged impurities absorbed on graphene or located between the graphene and the underlying substrate, to induce Coulomb scattering [13]. Evidence of graphene conductivity has been reported to be affected by many sources, such as interaction with the substrate [14], and ripples in suspended graphene [15]. 


\subsubsection{Thermal properties}

The low thermal conductivity of materials is a serious issue that has reduced the performance of electronic devices while generating heat when the device is operated. In contrast, graphene showed higher thermal conductivity in comparison to other carbon allotropes. For example, the suspended graphene monolayer has a thermal conductivity of 5,000 watts per meter per kelvin at room temperature, whereas the highest thermal conductivity of a single-wall carbon nanotube is around 3,000 W/mK [16]. However, graphene sheets supported with $\mathrm{SiO}_{2}$ have lower thermal conductivities $\sim 600 \mathrm{~W} / \mathrm{mK}$ near room temperature, due to phonon scattering of the substrate defects and impurities [17]. Moreover, the thermal conductivity of graphene changes according to the number of layers. As the thickness of the graphene increases, the phonon dispersion varies due to the availability of more phase-space states. Although this leads to phonon scattering, which reduces the thermal conductivity to the range of 1,000 to $2,000 \mathrm{~W} / \mathrm{mK}$, it remains higher than most semiconductor materials and metals [18].

\subsubsection{Mechanical properties}

While graphene offers valuable optical and electronic properties, its mechanical properties are equally impressive. Despite its one-atom thickness, graphene has been reported to be the strongest material ever measured [19]. Its breaking strength is about 42 newtons per meter $(\mathrm{N} / \mathrm{m})$, which is more than 100 times larger than that of a hypothetical steel film of equal thickness. In addition, graphene is the most flexible elastic membrane, as it can preserve more than $20 \%$ of local strain without breaking [19].

AFM has been commonly used for the study of graphene's elastic properties. In particular, the spring constant of suspended graphene was calculated by applying stress and using atomic force microscopy and determined to be $1-5 \mathrm{~N} / \mathrm{m}$ [20]. Moreover, an intrinsic strength of $130 \mathrm{GPa}$ and Young's modulus of 1TPa was found for single layer suspended graphene [19]. Thus, graphene is referred to as a 'magical' material that combines both strength and lightness in the same material that can be relatively controlled through the number of layers (Table1 3.1) [1]. 
Table 3.1: Mechanical properties of graphene [1].

\begin{tabular}{lll}
\hline Method & Material & Mechanical properties \\
\hline AFM & Mono layer graphene & $\begin{array}{l}E=1 \pm 0.1 \mathrm{TPa} \\
\sigma_{\text {int }}=130 \pm 10 \mathrm{GPa} \text { at } \varepsilon_{\text {int }}=0.25\end{array}$ \\
& \\
& & \\
\hline AFM & Mono layer & $E=1.02 \mathrm{TPa} ; \sigma=130 \mathrm{GPa}$ \\
& Bilayer & $E=1.04 \mathrm{TPa} ; \sigma=126 \mathrm{GPa}$ \\
& Tri-layer & $E=0.98 \mathrm{TPa} ; \sigma=101 \mathrm{GPa}$ \\
& Graphene & \\
\hline
\end{tabular}

Raman spectroscopy is another technique that has been used to investigate graphene's mechanical properties. For instance, tensile and compressive strain in graphene can be determined by changes in the $\mathrm{G}$ and $2 \mathrm{D}$ bands. A redshift was observed in the $2 \mathrm{D}$ band for a slight strain around $0.8 \%$, with redshift and splitting of the $\mathrm{G}$ band as the strain increased [21]. Moreover, a significant redshift of 2D peak was observed when introducing tensile strain to graphene sheets by bending the transparent and flexible substrate. The 2D peak could only be used to detect the strain if the G peak overlapped with a strong peak from the substrate. Graphene is capable of immediate recovery once the stress is released, whereas the carbon nanotubes (CNT) take over one week to recover due to slippage $[22,23]$. This remarkable elasticity of graphene is critical for the practical applications thereof, such as ultra-sensitivity of strain sensors [22].

Another force applied to graphene was the stretching of an elastic polymer substrate. This led to a significant red shift of 2D and G modes under uniaxial tensile strains [24], and the substrate partly induced the strain. It was observed that there are significant upshifts of all the Raman peaks attributed to the compressive strain caused by the $\mathrm{SiC}$ substrate. Specifically, epitaxial graphene on a SiC substrate (EG) resulted in a blueshift of bulk graphite and graphene made via mechanical exfoliation [25].

Table 3.2 summarises the changing Raman spectroscopy peaks based on various factors [1]. Chapter five investigates the strain in graphene evaluated in different temperatures and supporting morphology substrates using Raman spectroscopy results. The Raman spectroscopy bands in graphene were introduced and explained in Chapter two. 
Table 3.2: Effect on various factors on the Raman peak, shape, and splitting [1].

\begin{tabular}{|c|c|c|}
\hline Factors & Peak "G" & Peak " $2 D^{\prime}$ \\
\hline Graphene layers & - & Blue shift \\
\hline Tensile strain & Red shift & Red shift with stain \\
\hline Commraceisia os & Dad chift with etrain and enl & Rad chift with strain \\
\hline \multicolumn{3}{|l|}{$\mathrm{SiC})$} \\
\hline Temperature & Red shift with temperature & No change \\
\hline Doping & Blue shift with doping & - \\
\hline
\end{tabular}

\subsection{Morphology of graphene}

The structural properties of graphene are another important perspective that began to attract interest in 2007. It is stated that a presence of nm-sized ripples in single-layer graphene were found by studying electron diffraction on the suspended graphene membranes (Figure 3.5) [15]. They noted that the electron diffraction pattern becomes blurred when increasing the incident angle of the electron beam. For instance, broadening the diffraction peaks with the tilt angle could be understood due to microscopic corrugations on the graphene plane. They also found that the graphene ripples have an average height of about $1 \mathrm{~nm}$ with a lateral dimension in the range of $10 \mathrm{~nm}$ to $25 \mathrm{~nm}$. It has been found that the rippling is suppressed in thicker graphene samples. The nanoscale rippling is argued to be an intrinsic feature of graphene, essential in keeping the stability of its 2D structure $[15,26]$.

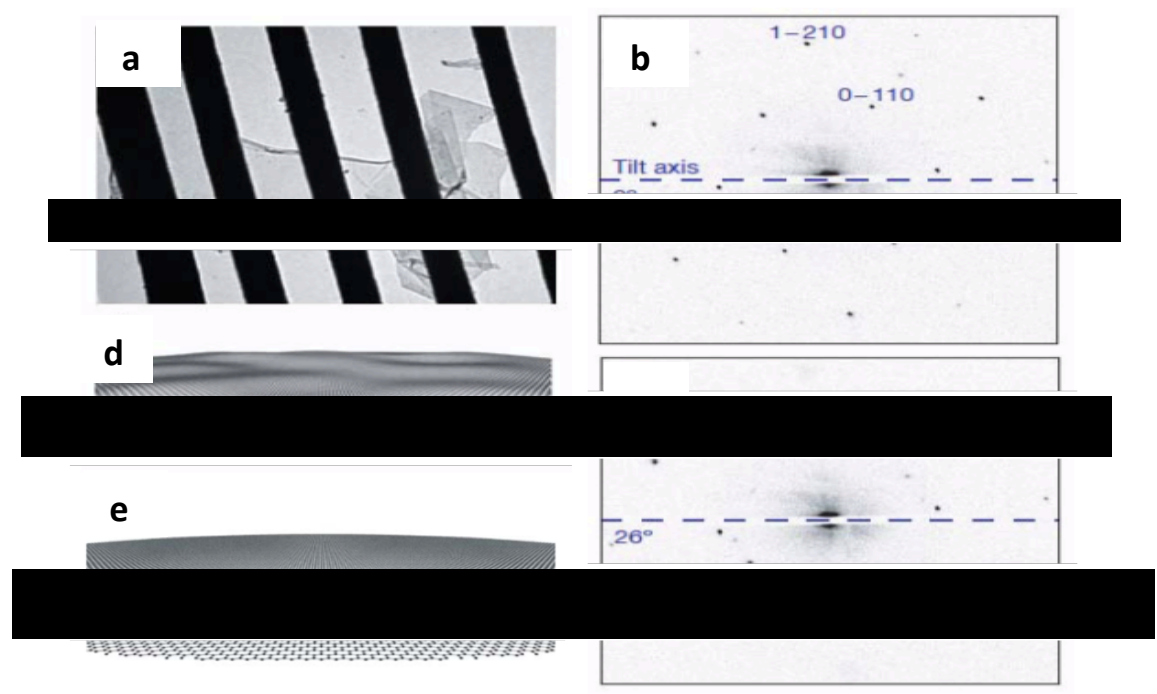

Figure 3.5: a) TEM image of suspended graphene membranes. (b)(c) Electron diffraction patterns from a graphene single layer under 
different incidence angle of 0 (b) and 26 (c) degrees. (d) Schematic for corrugated graphene (e) Flat graphene crystal [15].

In addition to the studies of free-standing graphene layers, many investigations have addressed how graphene properties are strongly affected by the interactions with its neighbouring materials. When graphene is deposited or manufactured on a substrate, the substrate morphology is one of the main factors that greatly affect graphene's properties. Particularly, the graphene morphology supported on rougher substrates shows low van der Waals interaction energy and high elastic energy between the two materials. This deforms the graphene resulting in mechanical failure and the breakdown of the electronic properties thereof [27]. As a result of this, the conformation of graphene was studied with several substrates [4, 28-30].

The presence of microscopic rippling of the 2D graphene sheets, either in free-standing form or supported with substrates, has attracted great interest in the scientific community. Furthermore, scanning probe microscopy was used to investigate the conformation of graphene with several substrates. These studies showed that the roughness of graphene depends on how it conforms to the substrate, meaning that flat substrates offer better roughness and graphene conformation. As the mica surface is at least five times smoother than a silicon dioxide substrate, it was suggested that graphene on $\mathrm{SiO}_{2}$ have $154 \mathrm{pm}$ of roughness, with $24 \mathrm{pm}$ for mica [28] as shown in figure 3.6. This atomically flat substrate has not shown any obvious ripples in graphene that cannot be avoided in $\mathrm{SiO}_{2}[28,4]$ and suspended graphene [15].

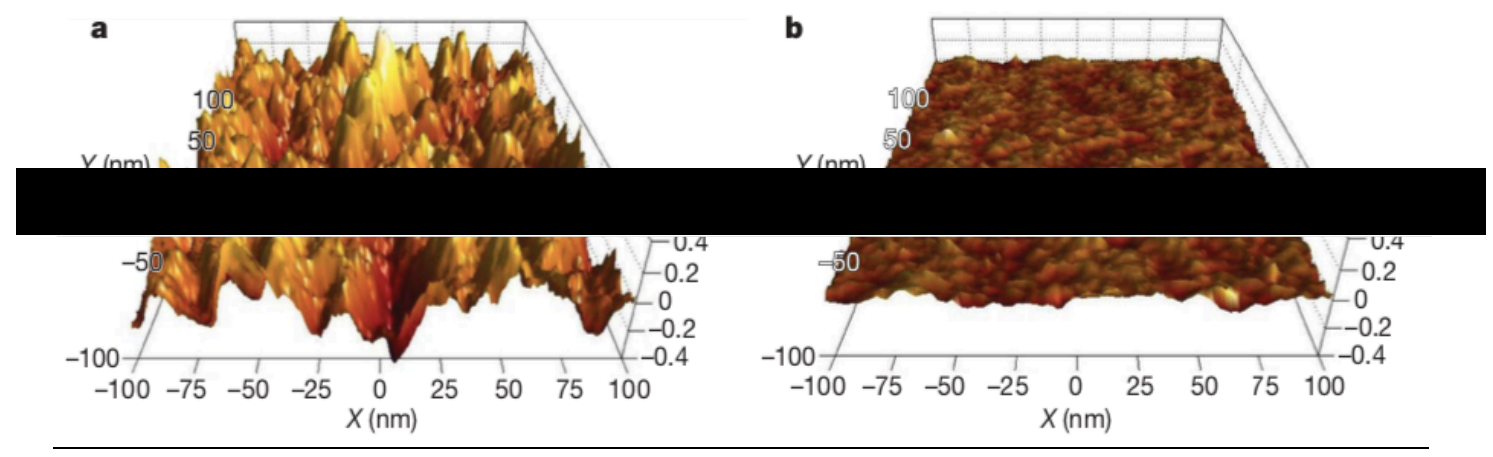

Figure 3.6: Graphene roughness on a) $\mathrm{SiO}_{2}$ b) mica substrates [28] 


\subsection{Methods for graphene production}

There are several methods to synthesise graphene layers, classified as either chemical or physical methods. The most used techniques include chemical synthesis, thermal chemical vapour deposition, chemical exfoliation, and mechanical exfoliation (Figure 3.7). Each of these approaches has both benefits and drawbacks.

The first method was achieved by Geim and co-workers. They used dry mechanical exfoliation of graphene with varying thicknesses by using graphite crystals as a starting material and adding either scotch tape or rubbing another material against the surface of the graphite $[2,3,31]$. Although this is the simplest approach, it is a technique that cannot be controlled and requires several days or weeks to locate an appropriately thin unfolded layer. In addition, it limited by low production with a small size for a single layer of $10 \mu \mathrm{m}$, being insufficient for industry purposes. Despite the disadvantages, this is still the most used approach for research, due to its simplicity and yielding of high-quality graphene.

In 2006, Somani and co-workers achieved the first successful few-layer graphene film using chemical vapour deposition (CVD). They used camphor on $\mathrm{Ni}$ foils that produced high-yield and high-quality graphene of $20 \mu \mathrm{m}$ [32]. This technique is based on the growth of graphene on flat transition metal substrates produced with carbonaceous gaseous sources with reactions at high temperatures around $1,100{ }^{\circ} \mathrm{C}$. The main problems with this approach are a strong adherence of the first graphene layer to the metal substrate and a high cost.
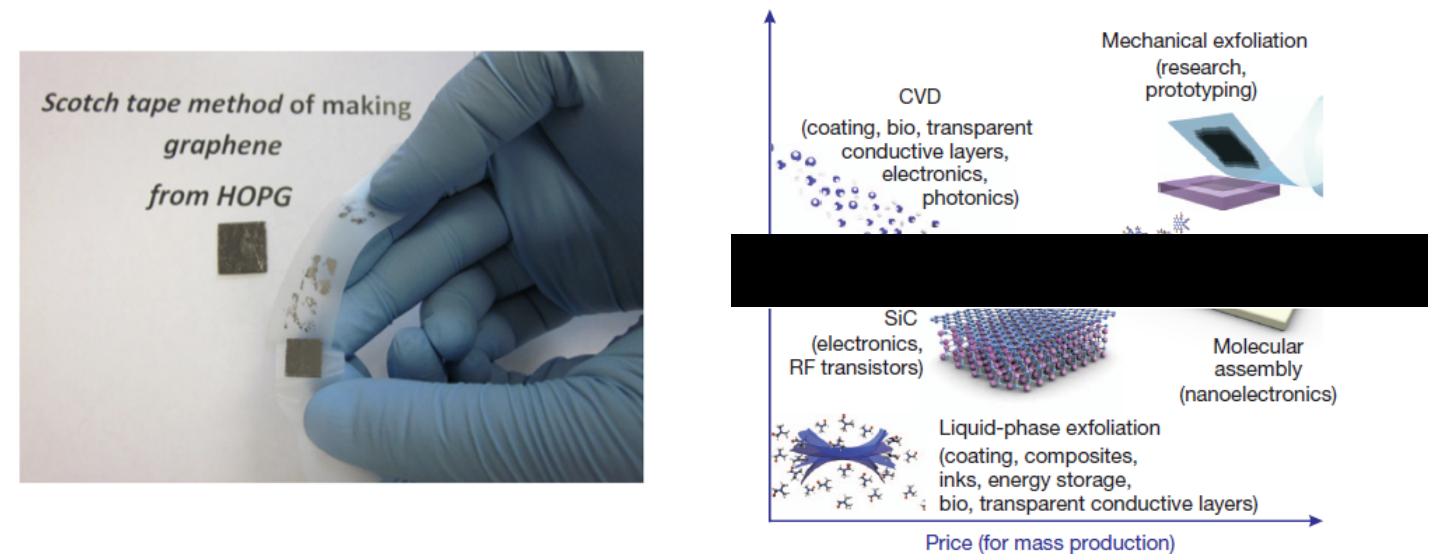

Figure 3.7: Left: Graphene synthesis via mechanical exfoliation. [1].

Right: several methods of graphene synthesis [10]. 
The formation of graphene on $\mathrm{SiC}$ is another common method. It is based on heating SiC substrates around $1,200{ }^{\circ} \mathrm{C}$ in UHV. The Si evaporates and leaves graphene layers behind on the $\mathrm{SiC}$ substrate [33]. The number of graphene layers can be controlled by both $\mathrm{SiC}$ pressure and temperature parameters. This is a simple method of formation single-crystal high-quality graphene. In addition, $\mathrm{SiC}$ is a large bandgap semiconductor already used in electronic applications without the requirement of transferring graphene to another substrate. This advantage overcomes the issue of the CVD method but could be a limitation as it is difficult to transfer graphene to other substrates. Moreover, this method struggles to produce uniform multilayers of graphene.

Finally, several other methods have been developed such as the reduction of graphene oxide [34], and molecular beam deposition [35]. Neither of these methods are expected to overtake the most common methods for future applications, being graphene on $\mathrm{SiC}$ and growth by CVD. However, the goal remains to provide an inexpensive, environmentally friendly, and high-yield production method for single and few-layers graphene.

\subsection{Graphene applications}

The potential properties of graphene have opened a new generation of electronics that offer flexible, light, strong, touch screen and transparent conductors with better performance compared to traditional devices. For instance, graphene is a potentially beneficial material that can be applied in the field of transistors. Particularly, highfrequency transistors based on SiC-graphene have high resistance at high temperatures when compared to traditional technology [10]. The combination of low resistance and high transparency provides an attractive option for using graphene in optoelectronic devices involving LED and smartphones. Additionally, graphene is used in the display of smartphone screens, ahead of ITO, which is expensive, scarce, and sensitive to breakage. As shown in Figure 3.8, Novoselov and his group provided an indicator of when a prototype working product could be planned based on project roadmaps and market leaders' production schedules [10]. 


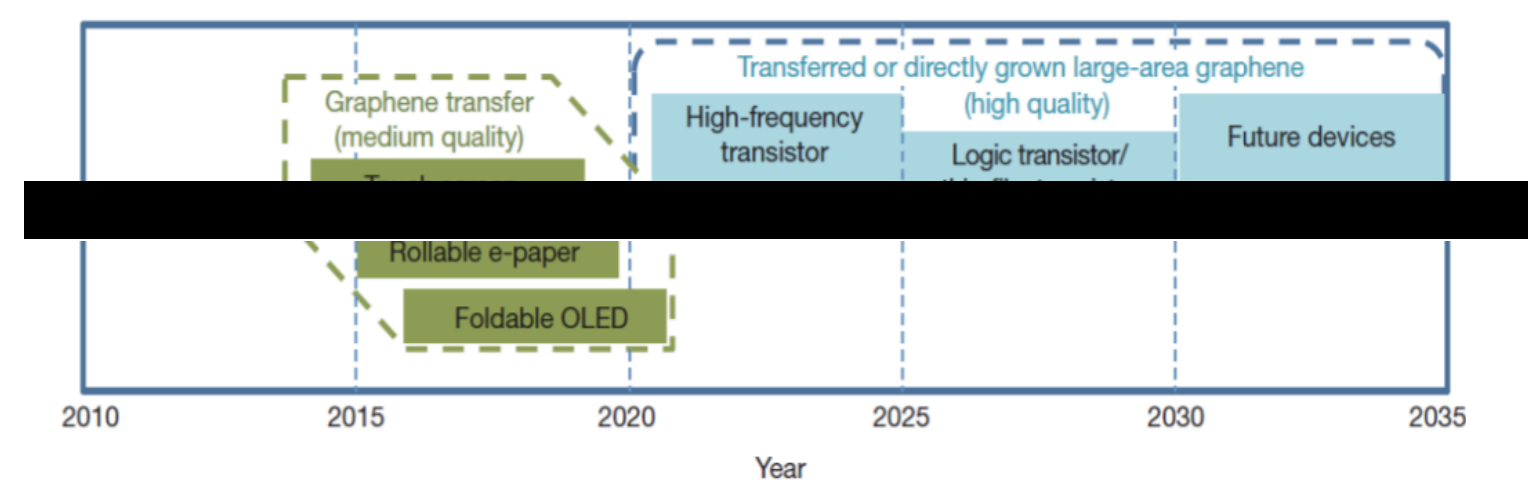

Figure 3.8: Graphene applications in various fields [10].

Because of graphene's flexibility, cost-effectiveness of manufacturing, and its ability to withstand great deformation of the plastic substrate, graphene is the ideal and strongest alternative material tested. Moreover, graphene could provide various kinds of flexible and stretchable electronic devices such as sensors [36]. Although graphene offers many advantages, its challenges include production yield with large conductivity, better adhesion with device substrates and stability under ambient conditions without any extra processing [36].

\subsection{Mica}

Mica belongs to the mineral family and is a homogenous inorganic natural substance. Typically, mica is divided into two groups based on its colour, where white includes muscovite and phlogopite, while dark is phlogopite and biotite.

Muscovite mica is an aluminosilicate with the chemical formula $\mathrm{KAl}_{2}\left(\mathrm{Si}_{3} \mathrm{Al}\right) \mathrm{O}_{10}$ $(\mathrm{OH})_{2}$. It consists of one layer of octahedrally coordinated $\mathrm{Al}^{+3}$ ions surrounded by two layers of tetrahedral $\mathrm{Si}^{+4}$ ions. Every fourth $\mathrm{Si}^{+4}$ ion is replaced by an $\mathrm{Al}^{+3}$, resulting in an excess negative charge, compensated by $\mathrm{K}^{+}$ions connecting the triple layers per Figure 3.9. In 1927, the structure of muscovite mica was first studied using X-ray diffraction [37, 38]. Muscovite mica was chosen by W.L. Bragg to investigate whether x-rays are very short electromagnetic waves that should be examined on a sufficiently flat surface to obtain regular reflection thereof [39]. 


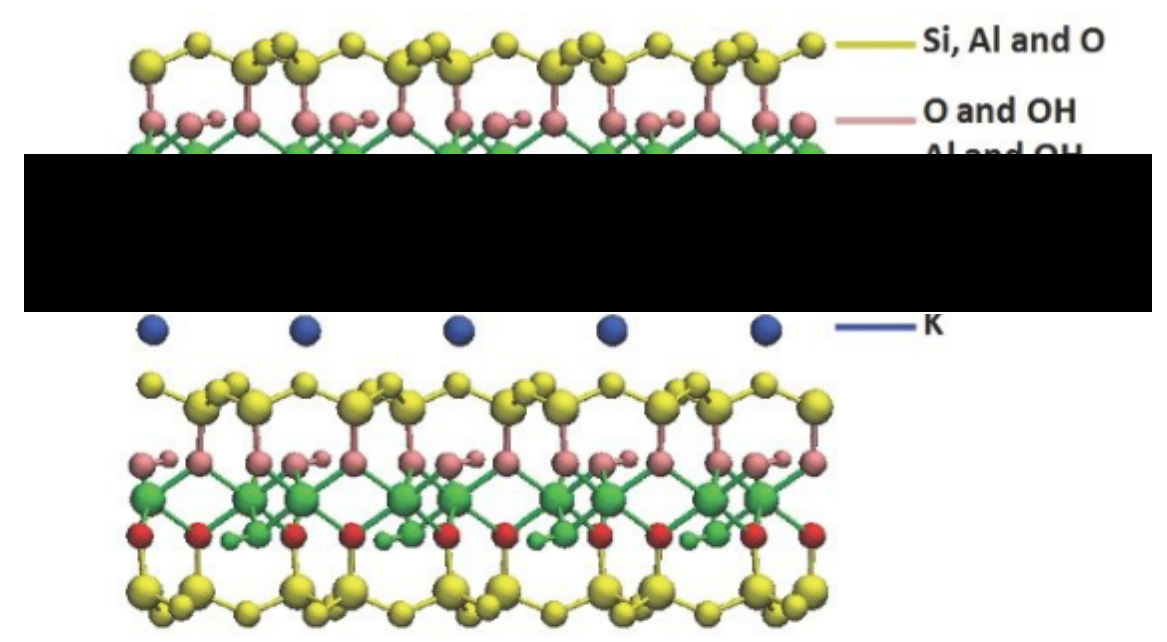

Figure 3.9: Crystal structure of muscovite mica [40]

\subsubsection{Mica properties}

In addition to its transparency, the most significant property of mica is elasticity, being simple and perfect cleavage. Due to these properties, mica is an appropriate substrate for the self-assembly of molecular layers [41-44], and to study thin films such as Pt [45]. Owing to its flatness, muscovite mica has proven many times better than ${\mathrm{a} \mathrm{SiO}_{2}}$ in terms of the observed graphene roughness $[46,28]$. In addition, mica has been used as a promising gate insulator substrate due to its low thermal and electrical conductivity, and high dielectric strength [47]. Notably, mica has been used as a reinforcing filler for thermosets and thermoplastics because of its impact on the physical, mechanical, and electrical properties of composites [48]. Mica has a Young's modulus of $172 \mathrm{GN} / \mathrm{m}^{2}$ versus $73 \mathrm{GN} / \mathrm{m}^{2}$ of glass flakes. Mica has remarkable chemical and corrosion resistance, good electrical properties, low thermal expansion, and causes much less wear and abrasion to the processing equipment [48].

\subsubsection{Applications of mica}

Muscovite mica is an extensively used material because of its transparency, heat resistance, and flatness. Due to its higher temperature resistance, light weight and thin design, mica is used in several applications, including the surface force apparatus, and creating a flat interface for organic solar cells [37].

Mica shows significant changes in dielectric properties of plastics when added to polymers. These changes in the electrical properties of mica-filled polymer composites make them useful in large-scale electrical insulation applications [48, 49]. In addition, 
the fabrication of large-area organic thin field-effect transistors based on a single mica crystal as a gate insulator - with a thickness of $<100 \mathrm{~nm}$ - has been reported for the first time in reference [50], demonstrating the great potential of mica ultrathin crystals as a flexible insulator for organic electronic devices.

\subsection{Summary}

Mica has been used for several years in insulation systems of high-power rotating machines. The two most used forms of mica are phlogopite and muscovite. Mica has a combination of physical, chemical, thermal, electrical, and mechanical properties not available from any other product, making it a significant compound in high voltage insulation systems. Mica can easily be cleaved into thin films while maintaining robust strength through the other two axes, making it simple to handle. In addition, it provides mica with its unique properties resulting from the structural arrangement of its atoms. In this chapter, the properties, methods of production, and applications of graphene were discussed. Graphene is the first two-dimensional (2D) material to illustrate stability under ambient conditions when deposited onto substrates. This property makes it an excellent material in various areas such as nano-mechanics, nanoelectronics, and other various areas that can benefit from its 2D membrane.

The most notable information in this chapter relates to the surface of mica being at least five times smoother than that of an $\mathrm{SiO}_{2}$ substrate. When placed on a flat mica terrace, graphene monolayers show a very flat structure, one of a kind from that found for graphene on $\mathrm{SiO}_{2}$. 


\subsection{References}

[1] Singh V, Joung D, Zhai L, Das S, Khondaker SI, Seal S. Graphene based materials: Past, present and future. Progress in Materials Science. 2011;56(8): 1178-1271. Permission no. 4799530000370

[2] Novoselov KS, Geim AK, Morozov SV, Jiang D, Zhang Y, Dubonos SV, et al. Electric field effect in atomically thin carbon films. Science. 2004;306(5696): 666-669.

[3] Novoselov KS, Jiang D, Schedin F, Booth TJ, Khotkevich VV, Morozov SV, et al. Two-dimensional atomic crystals. Proceedings of the National Academy of Sciences. 2005;102(30): 10451-10453.

[4] Yang G, Li L, Lee WB, Ng MC. Structure of graphene and its disorders: a review. Science and Technology of Advanced Materials. 2018;19(1):613-648. Available from: https://www.tandfonline.com/doi/full/10.1080/14686996.2018.1494493?tab=p ermissions\&scroll=top [Accessed 1st May 2020]. CC BY license.

[5] Geim AK, Novoselov KS. The rise of graphene. Nature Mater. 2007;6(3): 183191.

[6] Cheap Tubes. Allotropes of Carbon [Internet image]. Allotropes of Carbon. Available from: https://www.cheaptubes.com/screen-shot-2018-08-22-at-6-5012-pm/ [Accessed 5th July 2020]. Copyright (2020) by Cheap Tubes.

[7] Nair RR, Blake P, Grigorenko AN, Novoselov KS, Booth TJ, Stauber T, et al. Fine structure constant defines visual transparency of graphene. Science. 2008;320(5881): 1308 .

[8] Bonaccorso F, Sun Z, Hasan T, Ferrari AC. Graphene photonics and optoelectronics. Nature Photonics. 2010;4(9): 611.

[9] Roddaro S, Pingue P, Piazza V, Pellegrini V, Beltram F. The optical visibility of graphene: Interference colors of ultrathin graphite on SiO2. Nano Letters. 2007;7(9): 2707-2710. 
[10] Novoselov KS, Fal VI, Colombo L, Gellert PR, Schwab MG, Kim K. A roadmap for graphene. Nature. 2012;490(7419): 192-200. Permission no. 4795340715471.

[11] Xie G, Zhang K, Guo B, Liu Q, Fang L, Gong JR. Graphene-based materials for hydrogen generation from light-driven water splitting. Advanced Materials. 2013;25(28): 3820-3839. Permission no. 4894861424278.

[12] Bolotin KI, Sikes KJ, Jiang Z, Klima M, Fudenberg G, Hone J, et al. Ultrahigh electron mobility in suspended graphene. Solid State Communications. 2008;146(9-10): 351-355.

[13] Ando T. Screening effect and impurity scattering in monolayer graphene. Journal of the Physical Society of Japan. 2006;75(7): 074716.

[14] Hwang EH, Adam S, Sarma SD. Carrier transport in two-dimensional graphene layers. Physical Review Letters. 2007;98(18): 186806.

[15] Meyer JC, Geim AK, Katsnelson MI, Novoselov KS, Booth TJ, Roth S. The structure of suspended graphene sheets. Nature. 2007;446(7131): 60-63. Permission no. 4801600884535.

[16] Balandin AA, Ghosh S, Bao W, Calizo I, Teweldebrhan D, Miao F, et al. Superior thermal conductivity of single-layer graphene. Nano Letters. 2008;8(3): 902907.

[17] Seol JH, Jo I, Moore AL, Lindsay L, Aitken ZH, Pettes MT, et al. Twodimensional phonon transport in supported graphene. Science. 2010;328(5975): 213-216.

[18] Ghosh S, Bao W, Nika DL, Subrina S, Pokatilov EP, Lau CN, et al. Dimensional crossover of thermal transport in few-layer graphene. Nature Materials. 2010;9(7): 555-558.

[19] Lee C, Wei X, Kysar JW, Hone J. Measurement of the elastic properties and intrinsic strength of monolayer graphene. Science. 2008;321(5887): 385-388.

[20] Frank IW, Tanenbaum DM, van der Zande AM, McEuen PL. Mechanical properties of suspended graphene sheets. Journal of Vacuum Science \& Technology B: Microelectronics and Nanometer Structures Processing, Measurement, and Phenomena. 2007;25(6): 2558-2561. 
[21] Mohiuddin TMG, Lombardo A, Nair RR, Bonetti A, Savini G, Jalil R, et al. Uniaxial strain in graphene by Raman spectroscopy: G peak splitting, Grüneisen parameters, and sample orientation. Physical Review B. 2009;79(20): 205433.

[22] Yu T, Ni Z, Du C, You Y, Wang Y, Shen Z. Raman mapping investigation of graphene on transparent flexible substrate: the strain effect. The Journal of Physical Chemistry C. 2008;112(33): 12602-12605.

[23] Cronin SB, Swan AK, Ünlü MS, Goldberg BB, Dresselhaus MS, Tinkham M. Measuring the uniaxial strain of individual single-wall carbon nanotubes: Resonance Raman spectra of atomic-force-microscope modified single-wall nanotubes. Physical Review Letters. 2004;93(16): 167401.

[24] Ni ZH, Yu T, Lu YH, Wang YY, Feng YP, Shen ZX. Uniaxial strain on graphene: Raman spectroscopy study and band-gap opening. ACS Nano. 2008;2(11): 2301-2305.

[25] Ni ZH, Chen W, Fan XF, Kuo JL, Yu T, Wee ATS, et al. Raman spectroscopy of epitaxial graphene on a SiC substrate. Physical Review B. 2008;77(11): 115416.

[26] Fasolino A, Los JH, Katsnelson MI. Intrinsic ripples in graphene. Nature Materials. 2007;6(11): 858-861.

[27] Gao W, Huang R. Effect of surface roughness on adhesion of graphene membranes. Journal of Physics D: Applied Physics. 2011;44(45): 4.

[28] Lui CH, Liu L, Mak KF, Flynn GW, Heinz TF. Ultraflat graphene. Nature. 2009;462(7271): 339-341. Permission no. 4875290417197.

[29] Goncher SJ, Zhao L, Pasupathy AN, Flynn GW. Substrate level control of the local doping in graphene. Nano Letters. 2013;13(4): 1386-1392.

[30] Yamamoto M, Einstein TL, Fuhrer MS, Cullen WG. Charge inhomogeneity determines oxidative reactivity of graphene on substrates. ACS Nano. 2012;6(9): 8335-8341.

[31] Novoselov KS, Geim AK. The rise of graphene. Nature Mater. 2007;6(3): 183191.

[32] Somani PR, Somani SP, Umeno M. Planer nano-graphenes from camphor by CVD. Chemical Physics Letters. 2006;430(1-3): 56-59. 
[33] Berger C, Song Z, Li T, Li X, Ogbazghi AY, Feng, R, et al. Ultrathin epitaxial graphite: 2D electron gas properties and a route toward graphene-based nanoelectronics. The Journal of Physical Chemistry B. 2004;108(52): 1991219916.

[34] Pei S, Cheng HM. The reduction of graphene oxide. Carbon. 2012;50(9): 32103228.

[35] Zhan N, Olmedo M, Wang G, Liu J. Layer-by-layer synthesis of large-area graphene films by thermal cracker enhanced gas source molecular beam epitaxy. Carbon. 2011;49(6): 2046-2052.

[36] Jang H, Park YJ, Chen X, Das T, Kim MS, Ahn JH. Graphene-based flexible and stretchable electronics. Advanced Materials. 2016;28(22): 4184-4202.

[37] de Poel W, Pintea S, Drnec J, Carla F, Felici R, Mulder P, et al. Muscovite mica: Flatter than a pancake. Surface Science. 2014;619: 19-24. Available from: dx.doi.org/10.1016/j.susc.2013.10.008.

[38] Mauguin C. Étude des Micas au moyen des rayons X. Bulletin de la Société française de Minéralogie. 1928;51(5-8): 285-332.

[39] Bragg WL. The specular reflection of x-rays. Nature. 1912;90(250): 410.

[40] Yang L, Czajkowsky DM, Sun J, Hu J, Shao Z. Anomalous surface fatigue in a nano-layered material. Advanced Materials. 2014;26(37): 6478-6482. Permission no. 4793860236374.

[41] Verdaguer A, Sacha GM, Bluhm H, Salmeron M. Molecular structure of water at interfaces: Wetting at the nanometer scale. Chemical Reviews. 2006;106(4): $1478-1510$.

[42] van Hameren R, Schön P, Van Buul AM, Hoogboom J, Lazarenko SV, Gerritsen JW, et al. Macroscopic hierarchical surface patterning of porphyrin trimers via self-assembly and dewetting. Science. 2006;314(5804): 1433-1436.

[43] Frank P, Hlawacek G, Lengyel O, Satka A, Teichert C, Resel R, et al. Influence of surface temperature and surface modifications on the initial layer growth of para-hexaphenyl on mica (0 0 1). Surface Science. 2007;601(10): 2152-2160. 
[44] Kankate L, Balzer F, Niehus H, Rubahn HG. From clusters to fibers: Parameters for discontinuous para-hexaphenylene thin film growth. The Journal of Chemical Physics. 2008;128(8): 084709.

[45] Smith CP, Maeda M, Atanasoska L, White HS, McClure DJ. Ultrathin platinum films on mica and the measurement of forces at the platinum/water interface. The Journal of Physical Chemistry. 1988;92(1): 199-205.

[46] Rudenko AN, Keil FJ, Katsnelson MI, Lichtenstein AI. Graphene adhesion on mica: Role of surface morphology. Physical Review B. 2011;83(4): 045409.

[47] Lu XF, Majewski LA, Song AM. Electrical characterization of mica as an insulator for organic field-effect transistors. Organic Electronics. 2008;9(4): $473-480$.

[48] Deshmukh SP, Rao AC, Gaval VR, Joseph S, Mahanwar PA. Effect of particle size and concentration on mechanical and electrical properties of the mica-filled PVC. Journal of Minerals and Materials Characterization and Engineering. 2010;9(9): 831.

[49] Osman MA, Atallah A, Müller M, Suter UW. Reinforcement of poly(dimethylsiloxane) networks by mica flakes. Polymer. 2001;42(15): 65456556.

[50] He Y, Dong H, Meng Q, Jiang L, Shao W, He L, et al. Mica, a potential twodimensional crystal gate insulator for organic field-effect transistors. Advanced Materials. 2011;23(46): 5502-5507. 


\section{Chapter 4}

\section{Developing methods of mica preparation, graphene deposition and characterisation}

\subsection{Introduction}

Uniform and uncontaminated sample and substrate preparation methods are a requisite first step, followed by consistent and appropriate characterisation methods. This chapter details the development and testing of the mica and $\mathrm{SiO}_{2}$, substrate and graphene fabrication methods and the characterisation methods used.

\subsection{Experiments and results}

\subsubsection{Mica cleavage methods}

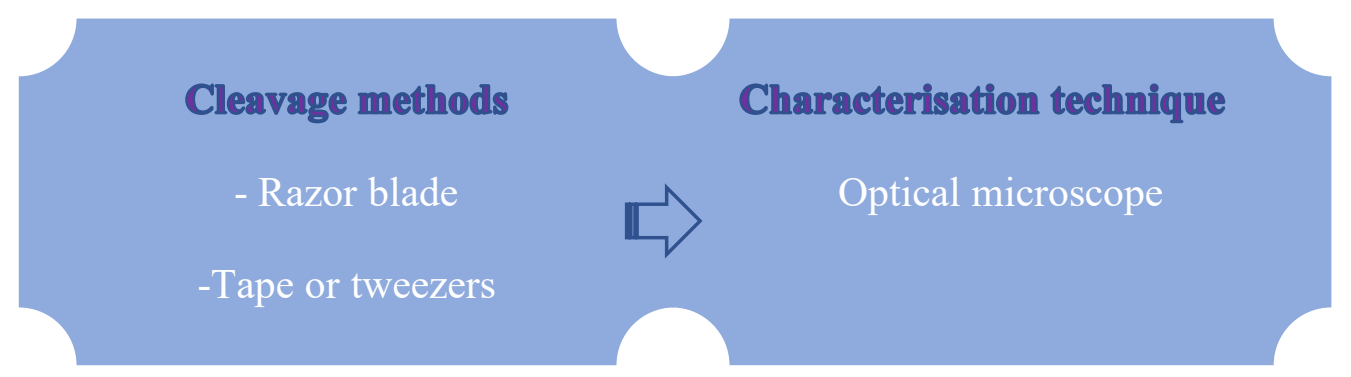

Stage 1: Study the effect of preparation methods on mica flatness

Muscovite mica $\mathrm{KAl}_{2}\left(\mathrm{Si}_{3} \mathrm{Al}\right) \mathrm{O}_{10}(\mathrm{OH})_{2}$ is easy to cleave along the $\{001\}$ planes, presenting large atomically flat terraces. The use of mechanical exfoliation has been shown to produce large single-layer graphene sheets on mica with characteristic lateral dimensions ranging from tens of micrometres to $0.2 \mathrm{~mm}$ [1]. Here, we apply two different cleavage methods on the highest quality of mica (Muscovite mica, Agar Scientific).

In the first method, mica is cleaved by a razor blade that has been cleaned using acetone and IPA solutions. This tool is used for a single cleavage, to reduce the possibility of contamination from any mica particles remaining on the blade. The middle of the razor blade is gently inserted into the corner, as close as possible to the mid thickness (the mica used is $0.2 \mathrm{~mm}$ thick with $9.9 \mathrm{~mm}$ diameter), avoiding any cutting or sawing of the mica, but instead, twisting the blade slightly, splitting the mica 
in two to initiate the cleave. In the second method, a sharp razor blade is used to make an incision in the corner of mica, then the mica is pulled using tape, and the crystal cleaves in half [2].

The results using optical microscopy (Zeiss) are presented in Figure 4.1. This shows that the second method produces a flatter, cleaner mica surface with fewer steps than the first method.

Figure 4.2 shows water drops, which are clearly observed in the razor blade section (but absent in other sections) in the tape-cleaved sample after several weeks. This may have happened because the blade produces an imperfect cleavage, which causes an inhomogeneous density distribution of potassium ions. This leads to hydrophilic properties that trigger the formation of potassium carbonate islands on the surface, if ambient water is present [3].

Due to this observation, the razor blade method was not used and the efficiency of the tape method confirmed. In addition, if the graphene sheet was deposited on one of the rough incision areas, later characterisation was unreliable, possibly because of the increased possibility of a trapped water layer between the graphene and the mica. The tape method was found to produce unequal halves of mica, one side being thicker than the other. The thinner portion is generally the tape side; usually the thicker portion is used, as it gives better contrast under microscopes and is easier to handle, thus the thinner half is disposed of. Subsequently, instead of using tape to pull the mica apart, the same initial cleave was performed with a razor blade, then tweezers were used to pull the two sections apart, which gave a better result and split the mica into almost equal halves. Both sections could then be used. 

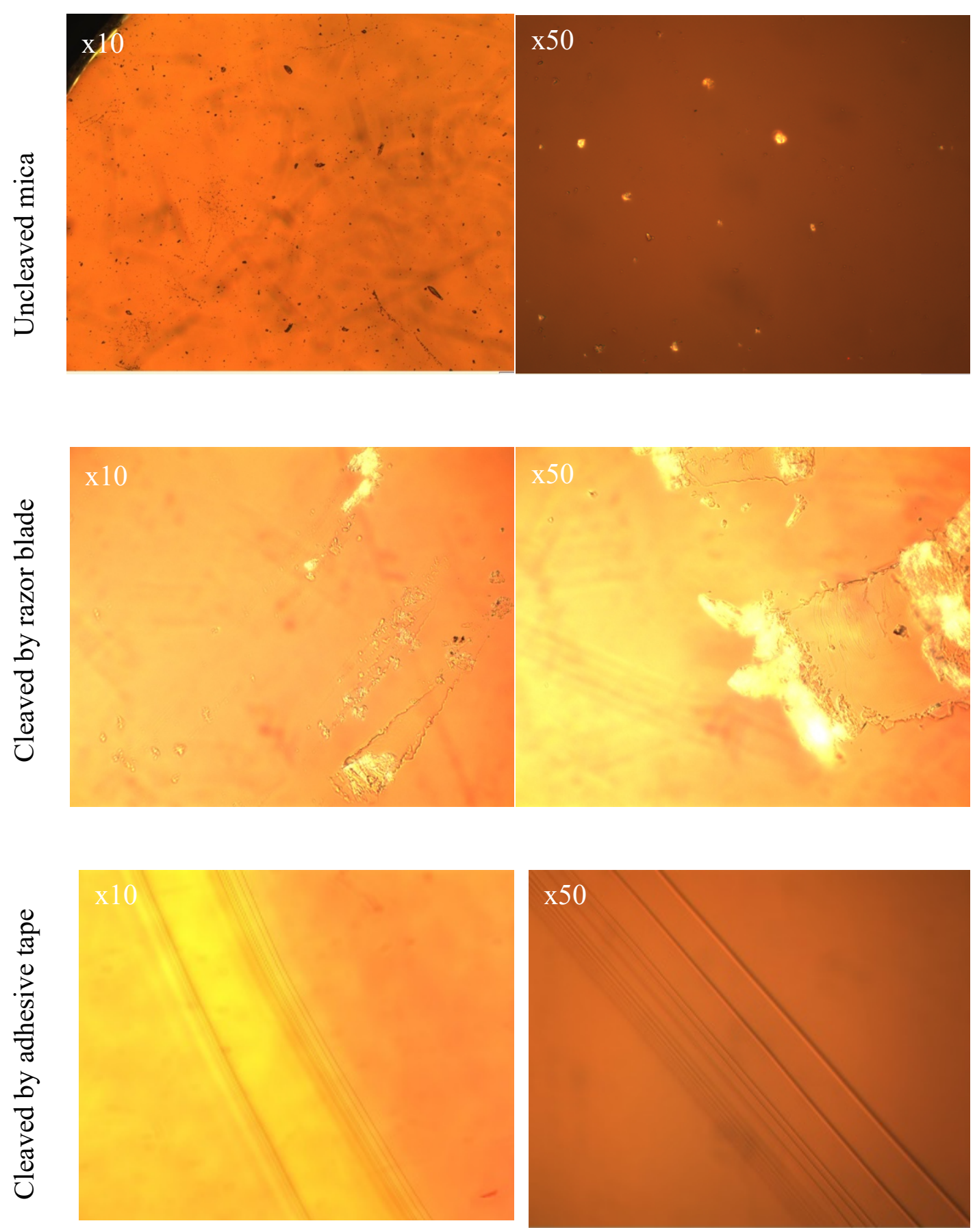

Figure 4.1: Optical microscopy images of mica before and after cleavage by razor blade and adhesive tape. Magnification: x10 and x50. 

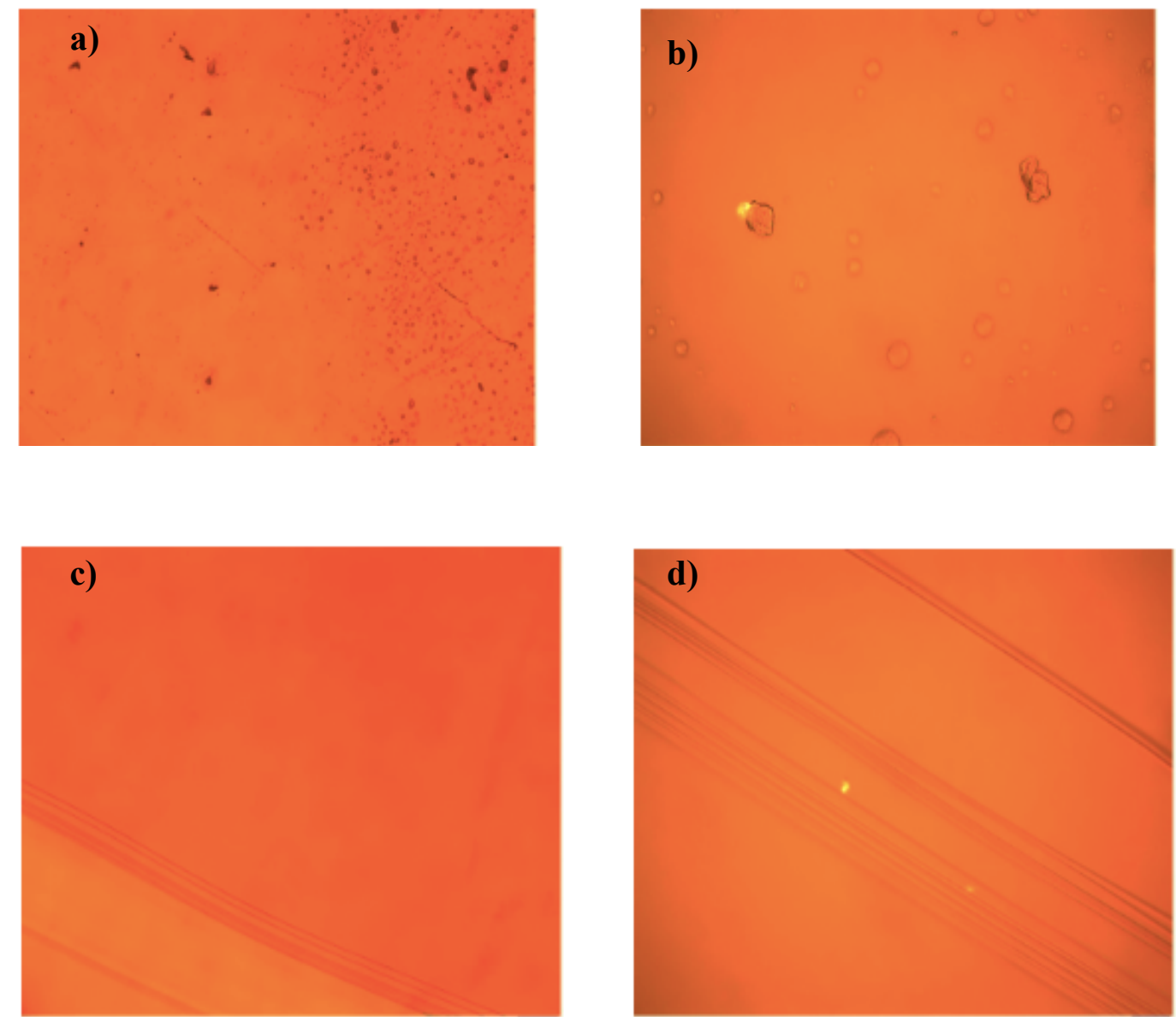

Figure 4.2: Optical microscopy images of different magnification. a) and b): water drops on cleaved mica after several weeks; c) and d): flat area in the same water sample. Magnification: x10 (left) and x50 (right).

\subsubsection{Graphene deposition methods on substrate}

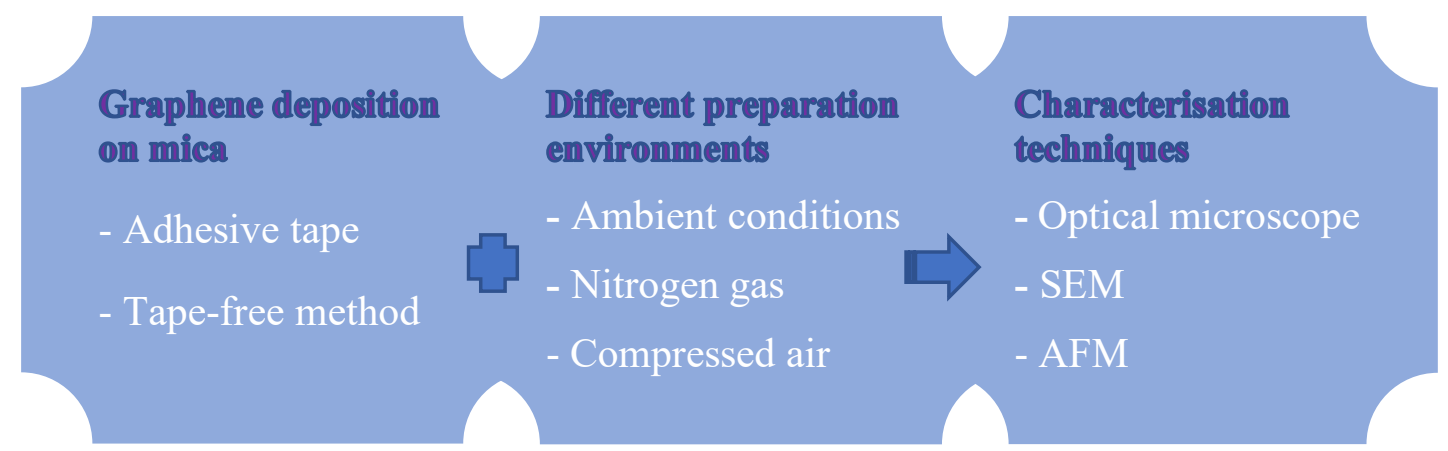

Stage 2: Characterisation of graphene sheets on mica substrate 
Methods of graphene production are explained in detail in Chapter 3. Here, two different methods of graphene deposition on a substrate will be examined, one using adhesive tape and one tape-free. Graphene sheets are produced by mechanical exfoliation of high-quality, highly oriented pyrolytic graphite (HOPG, grade ZYA, Agar Scientific). This HOPG exhibits an ABA (Bernal) stacking, therefore the multilayer exfoliated graphene sheets should follow the same stacking order. Before depositing graphene, the graphite should be prepared by using a new tape piece to remove any impurities and contamination that may occur on the graphite surface, such as hydrogen or water.

\section{A: Using adhesive tape}

The adhesive tape method was first used by Novoselov et al. [4] and has been adapted since, to simpler versions [5]. This method of depositing graphene on $\mathrm{SiO}_{2} / \mathrm{Si}$ substrate was also used in previous work done by this research group, but not on mica surfaces, and so was tested again here. Adhesive (Scotch) tape was placed on freshly cleaned graphite sample. It was pulled it off, then the tape was folded over so that graphite adhered to both sides. After that, the tape was again pulled back to separate the fragments into two, as shown in Chapter 3. This process was repeated many times to eventually obtain thin graphene layers. Next, a $\mathrm{SiO}_{2} / \mathrm{Si}$ substrate was dipped in this acetone solution, then washed in propanol solvent and sufficiently divested of water. Some of the exfoliated sheets were detected on the substrate's surface. Thick sheets were removed by cleaning the substrate in an ultrasonic bath, with propanol as a solvent, while the thin flakes remained attached to the $\mathrm{SiO}_{2} / \mathrm{Si}$ substrate.

In the case of graphene on mica substrate, the use of any solvent treatment that might perhaps cause changes in the mica's chemical composition was avoided. For this reason, first, the mica was simply cleaved using tweezers, as explained in Section 4.2.1. Then, in ambient conditions (relative humidity [RH] around 30\%), thin graphene flakes were immediately deposited onto the freshly cleaved mica substrate, to minimise the presence of a water layer between the graphene and the substrate $[1,6]$. The optical microscope image in Figure 4.3 shows the flat surface of the mica after cleavage; some contamination has appeared after graphene deposition because of the tape glue. However, when the sample was investigated on different days, the surface looked clean and no obvious evidence of any change was observed. 


\section{B: Tape-free method}

Another method that was used in 2005 by Novoselov et al., used a different technique where a fresh surface of the layered crystal was rubbed against the target surface, leaving a variety of flakes attached to it - the rubbing process can be described as similar to "drawing by chalk on a blackboard" [7].

This was investigated here too. Before starting the process, the HOPG was cleaned using a piece of tape, then the graphite was gently pressed onto the freshly cleaved mica surface. This has some advantages, including the prevention of any defects and contamination between the graphene and the mica. Another advantage of this method is that it avoids the mica peeling during the graphene deposition process, which may occur when scotch tape is used.

\section{C: Comparison and solution}

In this work, some graphene sheets were contaminated by the tape glue when using the adhesive tape method. Consequently, the tape-free method was initially preferred because it reduces these contamination effects. For the adhesive tape method, the mica surface was found to be much more sensitive than $\mathrm{SiO}_{2}$ to graphene exfoliation through the tape, because mica can be exfoliated at the same time as the graphene is deposited. However, the disadvantage of the tape-free method is that it produces much thicker graphene flakes. Although these can be washed by solvents after depositing on $\mathrm{SiO}_{2}$ (as mentioned above), it is not possible to use any solvent with mica because of chemical changes to the surface.
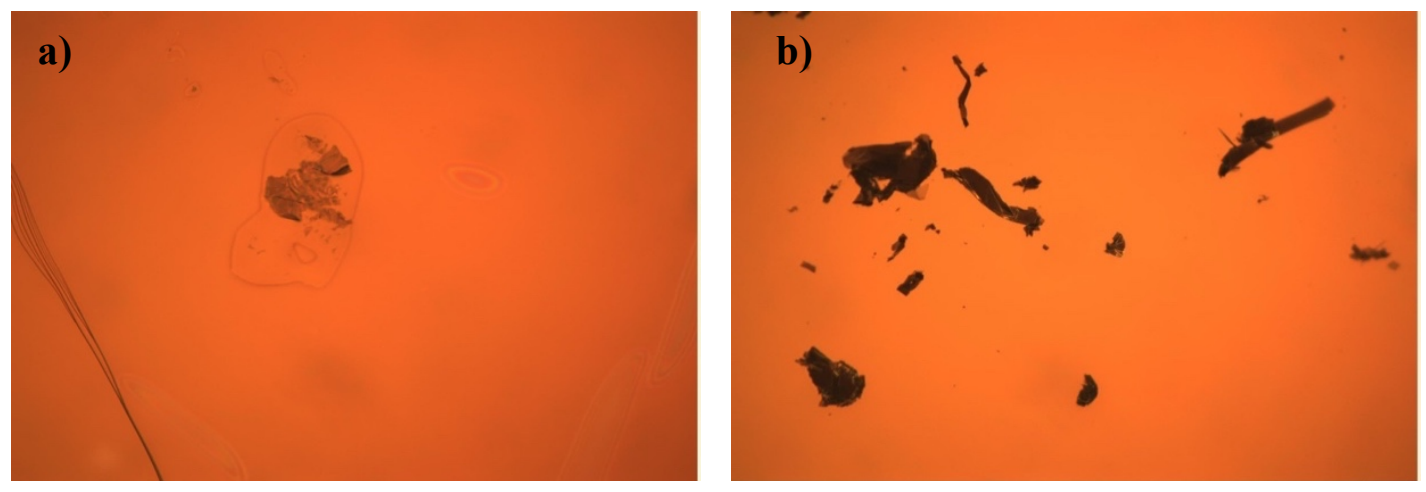

Figure 4.3: Optical microscopy (magnification: x50) of graphene on mica using different graphene production methods: a) adhesive tape method, b) tape-free method. 
In summary, therefore, the tape method produced better quality graphene film, but the tape left a residue. Mechanical exfoliation through rubbing produced thick clumps of graphene that could not be washed away. Thus, an alternative solution was devised using clean-room tape to replace Scotch tape. This adhesive is designed to support semiconductor wafers through manufacturing processes and dicing. This method follows the same procedure as the method of adhesive tape but replaces the common adhesive tape with the specialised wafer tape. Using this dicing type of tape was found to produce clean graphene flakes.

\subsubsection{Graphene on mica preparation environments}

In 2009, Lui et al achieved the first ultraflat graphene on mica without ripples, where sample preparation was carried out in a glove box with water and oxygen concentrations below 1 part per million (ppm), to minimise the presence of adsorbates at the graphene-mica interface [1]. Other works have used nitrogen or argon gas during graphene deposition onto mica inside a glove box $[3,6,8]$. Lui et al. states that despite the extremely flat graphene samples over areas on the sub-micron scale, differences within the topography may be seen when surveying the surface over distances of microns [1]. In particular, graphene topography shows the presence of flat plateaus that increase abruptly by a height of $\sim 0.4 \mathrm{~nm}$ above the lower regions, which are attributed to molecular adlayer islands trapped between the graphene and the mica. These islands may cause slight local roughness $\sigma$ from $0.0208 \mathrm{~nm}$ to 0.0041 $\mathrm{nm}$ for various regions of the graphene sample on mica [1]. Moreover, another study did not observe any differences in the number of elevations when using exfoliation in air, in a dry box or in an argon box with humidity below 2 ppm [3].

Based on this fact, in the present study, mechanical exfoliation on mica was investigated in three different preparation environments, including under ambient conditions ( $\mathrm{RH} 30 \% \pm 4$ ), using compressed air, and using nitrogen $\left(\mathrm{N}_{2}\right)$ gas in a glove bag with RH $0 \% \pm 4$. The three environments were compared using optical microscopy and AFM and no remarkable difference was found between the graphene on the mica samples. Consequently, it was concluded that graphene would be deposited on mica in air rather than in other environments. 


\subsubsection{Different characterisation techniques for the same area}

The dicing or clean-room tape method was considered to be better for producing thin graphene sheets. It was then decided that the deposition of graphene on mica would take place under ambient conditions, rather than in another environment. Subsequently, there were several techniques available to characterise the graphene sheets, including optical microscopy, SEM and AFM. Optical microscopy, as a fast, non-destructive technique, is beneficial to check the cleanness of the surface generally and to locate large areas of graphene sheets on the substrate. Graphene is only visible on the substrate in optical microscopy due to interference effects. SEM is more suitable to image individual graphene sheets, with the increased spatial resolution, while AFM provides information on graphene morphology and the thickness of the graphene sheets. Figure 4.4 shows three different techniques including a) optical microscopy, b) AFM and c) SEM used to characterise graphene sheets. The same graphene flake is used in all three techniques to offer a direct comparison between them.
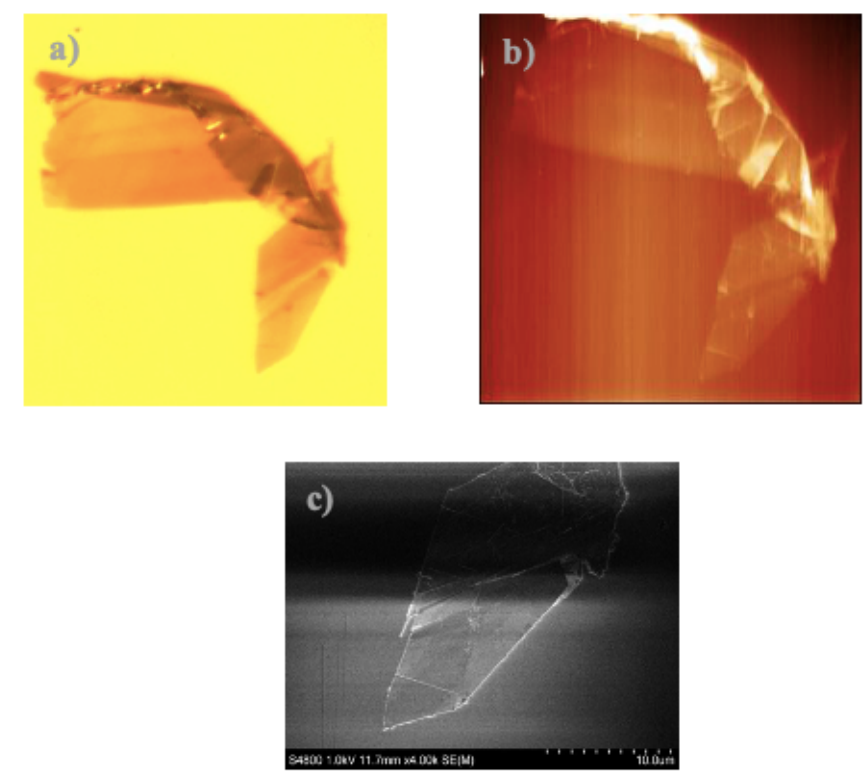

Figure 4.4: The same region under different characterisation techniques: a) optical microscopy with image size 60 $\mu \mathrm{m}$, b) AFM-contact mode with scan size $50 \mu \mathrm{m}$, and c) SEM. 


\subsubsection{Developing $\mathrm{SEM}$ techniques to image graphene on $\mathrm{SiO}_{2}$ and mica}

Here, the SEM technique is developed to demonstrate the best settings for imaging graphene sheets. When high acceleration voltages were tested, proportionally more secondary electrons were emitted from the substrate under the graphene than from the graphene itself, which reduced the apparent intensity of the graphene, essential to determine the difference in thickness between different graphene layers. Thus, graphene layers are more obviously detected at low acceleration voltages where incident electrons are less likely to penetrate the graphene and proportionally more secondary electrons are generated from the graphene compared to the substrate. Accordingly, the settings were first determined to give sufficient contrast to image few- and single-layer graphene, as shown in Figure 4.5. For mica the acceleration voltage was reduced to $2 \mathrm{kV}$, which also decreased charging effects from electron irradiation. The lower detector, which favours topographic information but is less sensitive to sample charging, was used in graphene on mica, whereas upper and mixed (upper/lower) detectors offered the highest contrast imaging for graphene on $\mathrm{SiO}_{2}$.
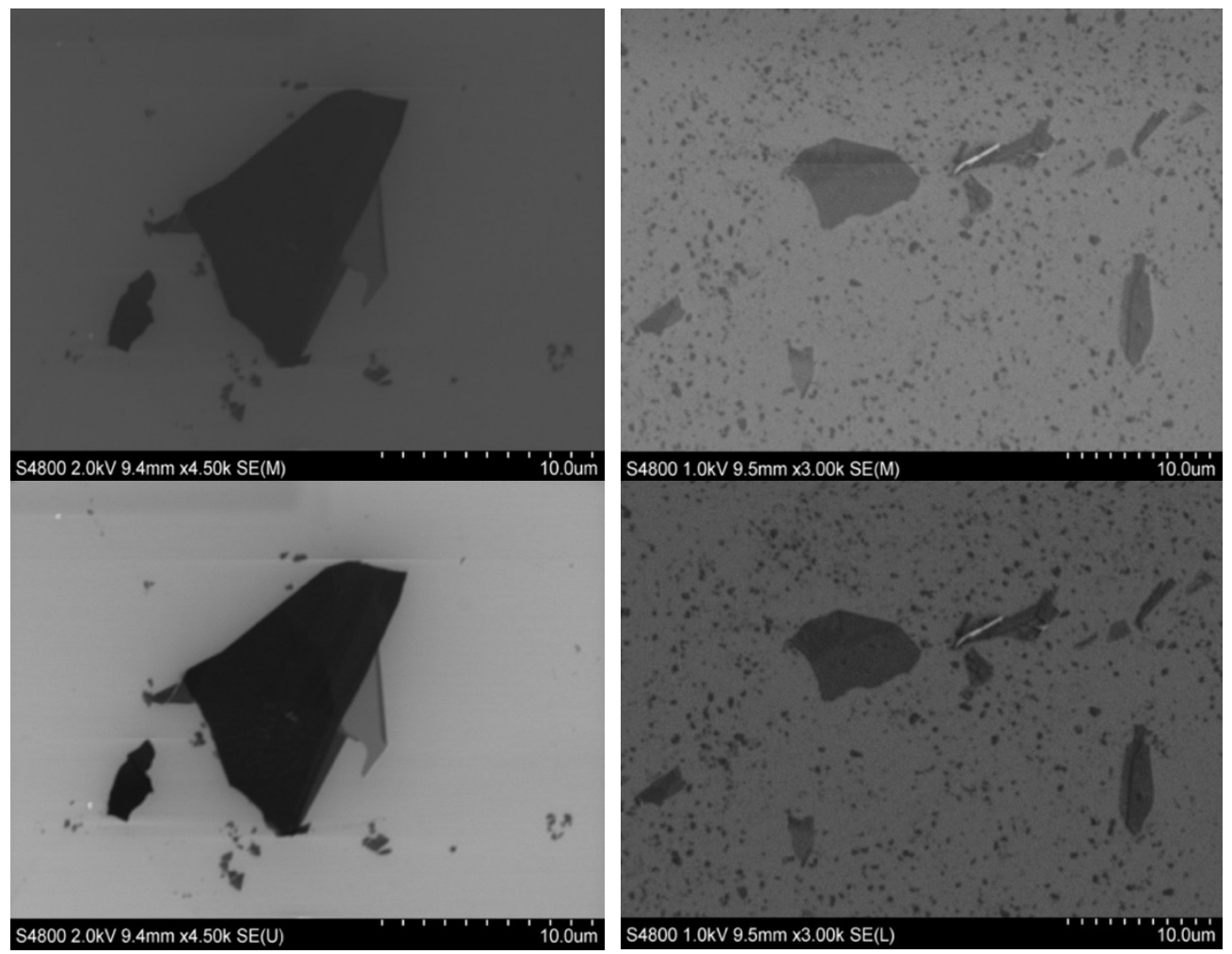

Figure 4.5: SEM image at different settings. Left: graphene on $\mathrm{SiO}_{2} / \mathrm{Si}$; right: graphene on mica. 
Thus, after concluding the investigation, SEM images of graphene samples were detected using a secondary electronic detector, at acceleration voltage $2 \mathrm{kV}$ and beam current $10 \mu \mathrm{A}$ for graphene on $\mathrm{SiO}_{2} / \mathrm{Si}$ using upper detector, and at acceleration voltage $1 \mathrm{kV}$ and beam current $5 \mu \mathrm{A}$ for graphene on mica using the lower detector; the working distance was estimated from $8 \mathrm{~mm}$ to $13.5 \mathrm{~mm}$.

\subsubsection{Thermal oxidation}

One of the main reasons why silicon is the most commonly used semiconductor material is the ability to form a chemically stable protective layer of silicon dioxide $\left(\mathrm{SiO}_{2}\right)$ on the surface of silicon [9]. The simplicity of fabricating $\mathrm{SiO}_{2}$ is another reason why $\mathrm{SiO}_{2} / \mathrm{Si}$ is the main substrate used in graphene devices.

Silicon thermal oxidation depends on the migration of oxidant species via the already formed $\mathrm{SiO}_{2}$ layer and following interfacial reactions. Thermal oxidation allows the formation of thin, practically defect-free, reliable $\mathrm{SiO}_{2}$ films. This is one of the reasons why silicon dominates the semiconductor industry, despite the presence of other semiconductive materials with better electrical properties than silicon [10].

The most common method for making an insulating $\mathrm{SiO}_{2}$ layer is via a thermal oxidation process, carried out inside a furnace in a stream of either oxygen $\left(\mathrm{O}_{2}\right)$ (dry oxidation via the reaction: $\mathrm{Si}+\mathrm{O}_{2} \rightarrow \mathrm{SiO}_{2}$ ) or water (wet oxidation via the reaction: $\mathrm{Si}+2 \mathrm{H}_{2} \mathrm{O} \rightarrow \mathrm{SiO}_{2}+2 \mathrm{H}_{2}$ ). Dry oxidation is slower but leads to denser oxides of higher quality [9], which encouraged the researchers to use dry oxidation to create the $\mathrm{SiO}_{2}$ layer on the Si substrate. This kind of oxidation method is generally used for metaloxide-semiconductor (MOS) gate oxides, while wet oxidation results in much faster growth and is typically used for thicker masking layers [9], because the water particles can separate at high temperatures to produce hydroxide $(\mathrm{OH})$, which can diffuse on the $\mathrm{Si}$ surface more rapidly than $\mathrm{O}_{2}$ molecules.

In the same way, the oxide growth rate is typically controlled by the temperature. For example, high temperatures are applied to increase the rate of oxidation, which means that more oxidants can reach the $\mathrm{Si} / \mathrm{SiO}_{2}$ interface and react with $\mathrm{Si}$ to produce $\mathrm{SiO}_{2}$.

Our research group has examined Si oxidation thickness and found that $90 \mathrm{~nm}$ is the best oxidation thickness to detect thin graphene, which matches with theoretical predictions [11]. Thus, in this work, the same settings and procedures are repeated, 
however, as the previous oxidation rig had been moved, a new rig was constructed which required re-calibration of the required oxidation time. $\mathrm{SiO}_{2}$ was grown in many wafers over various lengths of time, in order to determine the amount of time required to achieve the desired thickness, since the thickness of the $\mathrm{SiO}_{2}$ layer depends on the length of oxidation time in the furnace. Ellipsometry was then used to measure the thickness of the grown $\mathrm{SiO}_{2}$ layer. Spectroscopic ellipsometry (SE) is widely used to study thin films and complex-layered heterostructures with thickness parameters that have range from fractions of Angstroms to several micrometers [12].

In order to grow the $\mathrm{SiO}_{2}$ layer thermally, p-type boron-doped $\mathrm{Si}$ (100) wafers, oriented with silicon resistivity of $1 \Omega \cdot \mathrm{cm}$, were used (Figure 4.6). Then, the Si substrate was placed inside a quartz oxidation tube in a non-reactive atmosphere containing $\mathrm{N}_{2}$; the tube was loaded into the furnace centre and heated to $1,060^{\circ} \mathrm{C}$. The $\mathrm{N}_{2}$ was turned off and $\mathrm{O}_{2}$ was turned on when the optimum temperature was reached on the temperature controller. In this step, the $\mathrm{Si}$ can interact with $\mathrm{O}_{2}$ to procedure a $\mathrm{SiO}_{2}$ layer. When the desired time was reached, the $\mathrm{O}_{2}$ was turned off and the $\mathrm{N}_{2}$ was restarted for an hour, to let the furnace to cool [11].

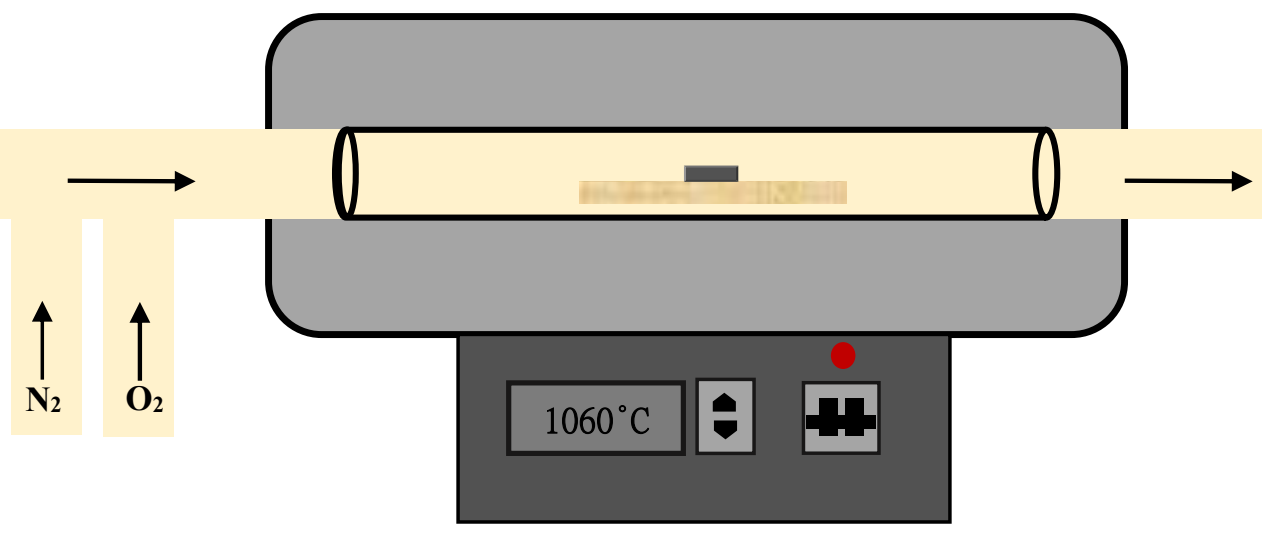

Figure 4.6: Thermal oxidation process.

The $\mathrm{SiO}_{2}$ layer was grown eight times at four different durations to define the amount of time required to achieve the necessary thickness. The thicknesses of the growing $\mathrm{SiO}_{2}$ layers were measured externally using ellipsometry.

Data were fitted in Matlab using a least-squares method to the function

$$
\frac{x_{0}}{A / 2}=\left[1+\frac{t+\tau}{A^{2} / 4 B}\right]^{\frac{1}{2}}-1
$$


In accordance with the Deal-Grove model, $t$ is the time (in hours) required to grow an oxide thickness of $x_{0}$ micrometres, $\tau$ is a time shift constant for the initial oxide layer, and $A$ and $B$ are constants [13]. The resulting fit is overlaid in figure 4.7 which shows the most significant result sought is the amount of time required to create a layer of $90 \mathrm{~nm}$ thickness. Solving the fitted equation shows that this thickness can be achieved with an oxidation time of 24 minutes.

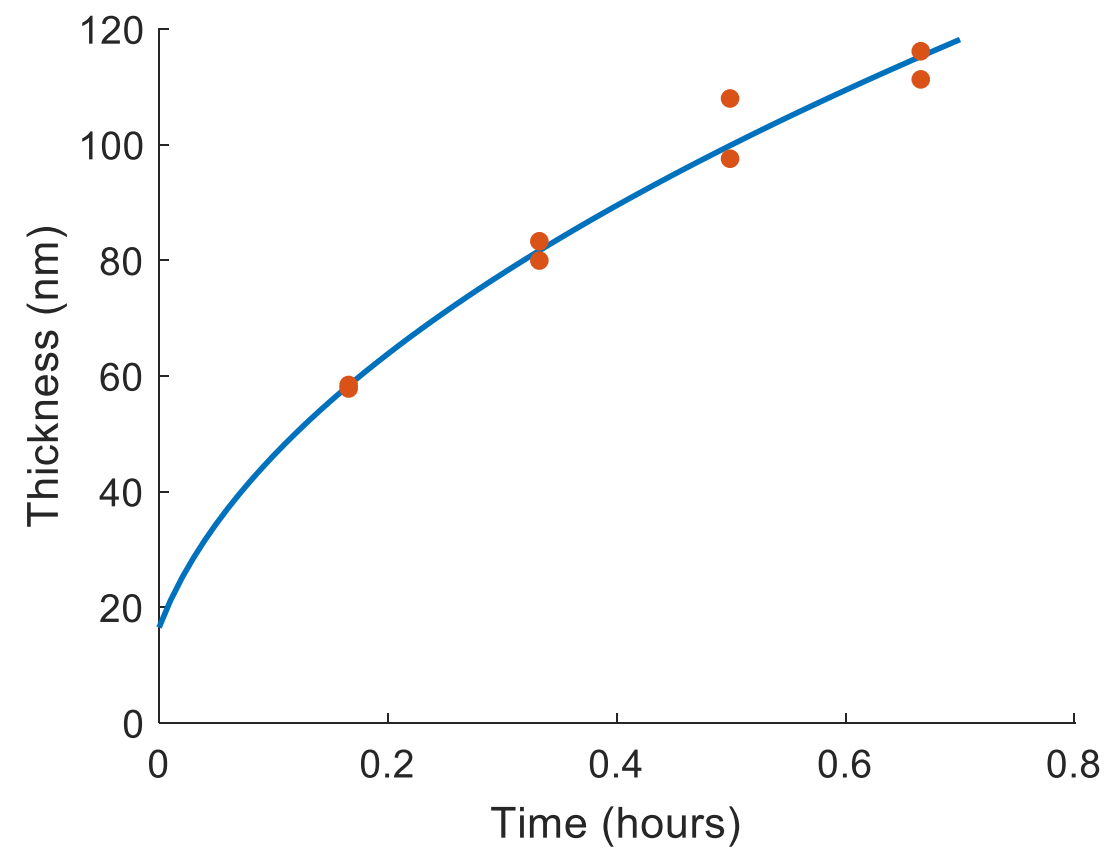

Figure 4.7: $\mathrm{SiO}_{2}$ thickness (measured using ellipsometry) as a function of oxidation time, fitted using a least-squares method.

\subsection{Summary}

Sample cleanliness and preparation method are considered an important stage for all types of scientific research, especially in the study of properties at the molecular level, where contaminants severely alter the final result. Hence, this chapter examines different preparation methods, including examination of mica cleavage. It was found that performing the initial cleave by making an incision in the corner of the mica, then pulling it apart using tweezers, gave the best clean flat mica surface. Two methods of graphene production were also examined. The tape method gave thin graphene sheets but the tape glue caused residue, while the tape-free method gave clean graphene sheets but produced thick clumps of graphene that could not be washed away using solvents. Scotch tape was therefore replaced by clean-room tape, as an alternative 
solution. Whether preparation of graphene on mica took place within a dry environment or under ambient conditions, no difference was found in the number of elevations attributed to molecular adlayers on the surface of the mica under the graphene sheet. Thus, for the later experiment, preparation of graphene on mica took place under ambient conditions.

Furthermore, SEM techniques to image mica on $\mathrm{SiO}_{2}$ and mica substrates were developed, to choose the best settings for detecting graphene sheets. In addition, comparison was made of imaging techniques (optical/AFM/SEM) for multi-layer graphene. Finally, the growth of oxide on silicon was tested under various growth times, for the depositing of an oxide layer of $90 \mathrm{~nm}$ thickness on silicon substrate, for further deposition of graphene sheet on $\mathrm{Si} / \mathrm{SiO}_{2}$. 


\subsection{References}

[1] Lui CH, Liu L, Mak KF, Flynn GW and Heinz TF. Ultraflat graphene. Nature. 2009;462(7271): 339-341.

[2] de Poel W, Pintea S, Drnec J, Carla F, Felici R, Mulder P, et al. Muscovite mica: Flatter than a pancake. Surface Science. 2014;619: 19-24. Available from: dx.doi.org/10.1016/j.susc.2013.10.008.

[3] Hattendorf S, Georgi A, Liebmann M, Morgenstern M. Networks of ABA and $\mathrm{ABC}$ stacked graphene on mica observed by scanning tunneling microscopy. Surface Science. 2013;610: 53-58.

[4] Novoselov KS, Geim, AK, Morozov SV, Jiang D, Zhang Y, Dubonos SV, et al. Electric field effect in atomically thin carbon films. Science. 2004;306(5696): 666-669.

[5] Hiura H, Miyazaki H, Tsukagoshi K. Determination of the number of graphene layers: Discrete distribution of the secondary electron intensity stemming from individual graphene layers. Applied Physics Express. 2010;3(9): 095101.

[6] Goncher SJ, Zhao L, Pasupathy AN, Flynn GW. Substrate level control of the local doping in graphene. Nano Letters. 2013;13(4): 1386-1392.

[7] Novoselov KS, Jiang D, Schedin F, Booth TJ, Khotkevich VV, Morozov SV, et al. Two-dimensional atomic crystals. Proceedings of the National Academy of Sciences. 2005;102(30): 10451-10453.

[8] Yamamoto M, Einstein TL, Fuhrer MS, Cullen WG. Charge inhomogeneity determines oxidative reactivity of graphene on substrates. ACS Nano. 2012;6(9): 8335-8341.

[9] Razeghi M. Technology of Quantum Devices. New York: Springer; 2010. p. 4182.

[10] Gerlach G, Maser K. A self-consistent model for thermal oxidation of silicon at low oxide thickness. Advances in Condensed Matter Physics. 2016: 1-13. Available from: doi.org/10.1155/2016/7545632. 
[11] Alyobi M. Local probe electrostatic manipulation of graphene: a study of the electronic and mechanical properties of rippled graphene sheets [Doctoral dissertation]. Swansea, UK: Swansea University; 2017.

[12] Kilic U, Mock A, Sekora D, Gilbert S, Valloppilly S, Ianno N, Langell M, Schubert E, Schubert M. Precursor-surface interactions revealed during plasmaenhanced atomic layer deposition of metal oxide thin films by in-situ spectroscopic ellipsometry. Scientific reports. 2020 Jun 25;10(1):1-2.

[13] Deal BE, Grove AS. General relationship for the thermal oxidation of silicon. Journal of Applied Physics. 1965;36(12): 3770-3778. 


\section{Chapter 5}

\section{Effect of thermal annealing and substrate morphology on the mechanical properties of graphene}

\subsection{Introduction}

Graphene's properties are strongly influenced by a number of factors, including its fabrication procedure and its interaction with the neighbouring material. The substrate morphology is one of the major factors that greatly affects the properties of graphene. As discussed in more detail in Chapter 3, it has been found that as the van der Waals interaction energy reduces between graphene and high-roughness substrates, the elastic strain energy is increased, which causes deformation in the graphene sheets $[1,2]$. Subsequently, the change in the mechanical properties of graphene causes change in its electronic properties. In particular, corrugations lead to charge inhomogeneity, scattering and low carrier mobility, which significantly impact the efficiency of electronic devices fabricated via graphene supported on $\mathrm{SiO}_{2}$ substrates [3].

From this perspective, a lot of research in recent years has addressed the effects of surface roughness by comparing the topography of graphene on many dielectric substrates [4-6]. It has been demonstrated that graphene morphology is free of ripples when supported on an ultraflat substrate such as mica. Similarly, experimental observation has shown that graphene on $\mathrm{SiO}_{2}$ is five times rougher than that on mica. This study opens up a new opportunity for the scientific community to consider mica as a promising alternative substrate to $\mathrm{SiO}_{2}[4]$.

Annealing is another factor that significantly affects graphene properties as part of the process of fabrication. Thermal annealing in different environments at various temperatures has been used to reduce or remove fabrication contamination and clean graphene to improve its quality [7]. However, this annealing process affects the properties of graphene, e.g. inducing morphological changes and directly inducing impurity doping [8]. In addition, annealing graphene in an oxygen-free atmosphere has been discovered to produce very broad extra Raman peaks overlapping the $\mathrm{D}, \mathrm{G}$ and $2 \mathrm{D}$ peaks of the graphene itself. These new Raman peaks are attributed to 
amorphous carbon produced on the surface of the graphene through carbonisation of environmental hydrocarbons [9]. Another example is work from the present research group, which demonstrates that annealing graphene on $\mathrm{SiO}_{2}$ under ultra-high vacuum (UHV) conditions causes an increase in the defect density and compressive strain of graphene induced from the substrate morphology [10]. However, although annealing causes modification in the electronic and structural properties of graphene, it is still needed, to clean the graphene surface. Therefore, the examination of annealed graphene on an alternative substrate has the potential to minimise the issue of annealing for graphene devices.

These substrate and annealing effects are both compounded by the thickness of the graphene. It is known that the structure of graphene materials is responsible for their physical properties; in particular the thickness has a notable effect. For instance, one theoretical study shows that the electrical conductivity of few-layer graphene decreased as the thickness increased, and that the reduction of conductivity was mainly caused by the inhibited carrier mobility [11]. This is consistent with an experimental study that shows the thickness dependence of the resistivity of individual graphene layers as low resistivity values for single strips and demonstrates that the resistivity distribution for single strips is anomalously narrow when compared to bi- and tri-layer graphene, consistent with the unique electronic properties of single graphene layers [12]. Hence, it is important to accurately determine the thickness and morphology of the graphene samples under different conditions, such as in the annealing process.

To date, however, there has been no direct comparison of the effects of annealing graphene on these two substrates $\mathrm{SiO}_{2}$ and mica. This chapter therefore investigates this, using Raman spectroscopy to detect the disorder in graphene structure and its electronic and mechanical properties. This experiment also helps to determine the optimal temperature for cleaning graphene sheets on $\mathrm{SiO}_{2}$ and mica substrates. In addition, it demonstrates the changing mechanical properties of graphene following annealing.

\subsection{Experimental methods}

Graphene sheets were obtained by mechanical exfoliation of high-quality, highly oriented pyrolytic graphite (HOPG) using the adhesive tape method in ambient conditions (typically measured RH $30 \% \pm 4$ ). These graphene flakes were deposited 
directly onto two substrates with different surface morphologies: freshly cleaved highquality muscovite mica and $90 \mathrm{~nm}$ of $\mathrm{SiO}_{2}$ on $\mathrm{Si}$. Then, the annealing processing was carried out in an ultra-high vacuum (UHV) at $200{ }^{\circ} \mathrm{C}, 300^{\circ} \mathrm{C}, 400^{\circ} \mathrm{C}$ and $500{ }^{\circ} \mathrm{C}$, for an hour.

Thin graphene sheets were identified using SEM, for graphene on mica at $1 \mathrm{kV}, 5 \mu \mathrm{A}$ and for graphene on $\mathrm{SiO}_{2} / \mathrm{Si}$ at $2 \mathrm{kV}, 10 \mu \mathrm{A}$. The layer number and the quality of each sheet were determined by Raman spectroscopy before and after each annealing step at laser excitation wavelength of $532 \mathrm{~nm}$, with the laser power below $1 \mathrm{~mW}$ to prevent heating the samples (this is important to avoid inducing a shift of the G peak). Each Raman spectrum was an average of 6 accumulations to improve the signal-to-noise ratio, the measurement time was 10 seconds and the maximum 100x objective lens was used to focus the laser beam onto the graphene sample. A Raman signal arises not only from the graphene sample, but also from the substrate, which is referred to as the background signal or simply background. To make spectra clearer and improve identification of sample peaks, the background is removed. Here, this is performed in the instrument software WiRE using the Subtract Baseline option. The software contains proprietary algorithms to subtract the background without altering the detected peaks. Then the resulting data from the sample were fitted and analysed using Fityk software. Each Gaussian peak was fitted by manually selecting a position to add a peak start position; for the Raman peaks G, D and 2D. All the manuallyidentified peak centre positions were simultaneously fitted by a genetic algorithm method. Contact mode AFM was used to measure the structure of the layers, to confirm the thickness of graphene sheets and investigate the height difference between pre-annealing and post-annealing stages.

\subsection{Results and discussion}

\subsubsection{Pre-annealing stage}

This section will establish the base properties of the graphene-on-substrate samples before annealing. For clarity, the same four representative sample sites - two on mica, two on $\mathrm{SiO}_{2}$ - are used throughout the chapter, with reference to other samples where needed. 
Figure 5.1 shows SEM images of three graphene samples, on (a) $\mathrm{SiO}_{2}$ and (b) mica. The mechanical exfoliation technique, described above, produces graphene of higher quality than other techniques [13], but it can lead to folded sheets. However, sheets folded over on themselves are valuable to allow a direct comparison of the unfolded 'thin' section and folded 'thick' section, which will always be double the layer thickness of the thin region; this is applicable here to the samples in Figure 5.1 (a). This gives four sample areas to study: on $\mathrm{SiO}_{2}$, 'thick' graphene (S1) and 'thin' graphene (S2), and (b) on mica, thick graphene (M1) and thin graphene (M2). The extra folded graphene fragment on the right in Figure 5.1 (a) has been left in deliberately to aid alignment and discussion of post-annealing AFM later.

(a)

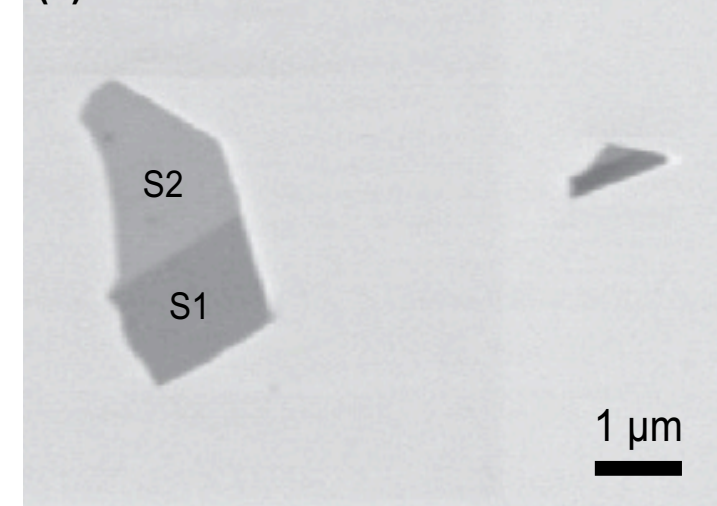

(b)

M2

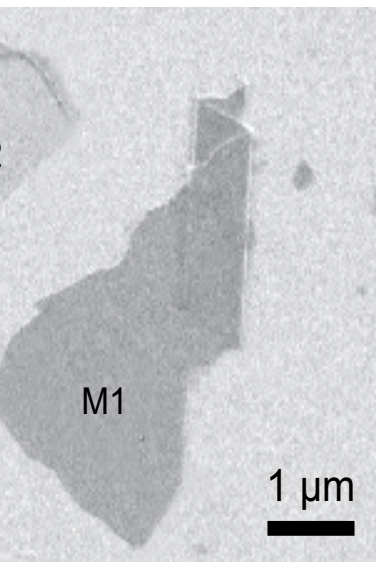

Figure 5.1: SEM image pre-annealing: a) 'thick' $S 1$ and 'thin' $S 2$ graphene attached to $\mathrm{SiO}_{2} / \mathrm{Si}$ substrate and b) 'thick' $\mathrm{M} 1$ and 'thin' M2 graphene on mica substrate.

The number of graphene layers in each of these four samples was determined using Raman spectroscopy, as shown in Figure 5.2. There are three principal features in Raman spectra of graphene that can be used to assess the number of layers in graphene: (i) the ratio of the intensity of the $2 \mathrm{D}$ band to the intensity of the $\mathrm{G}$ band, $\mathrm{I}_{2 \mathrm{D}} / \mathrm{I}_{\mathrm{G}}[14]$; (ii) the full width at half maximum (FWHM) of the 2D band [15] and (iii) the positions of the $\mathrm{G}$ and $2 \mathrm{D}$ bands $[14,16]$. 


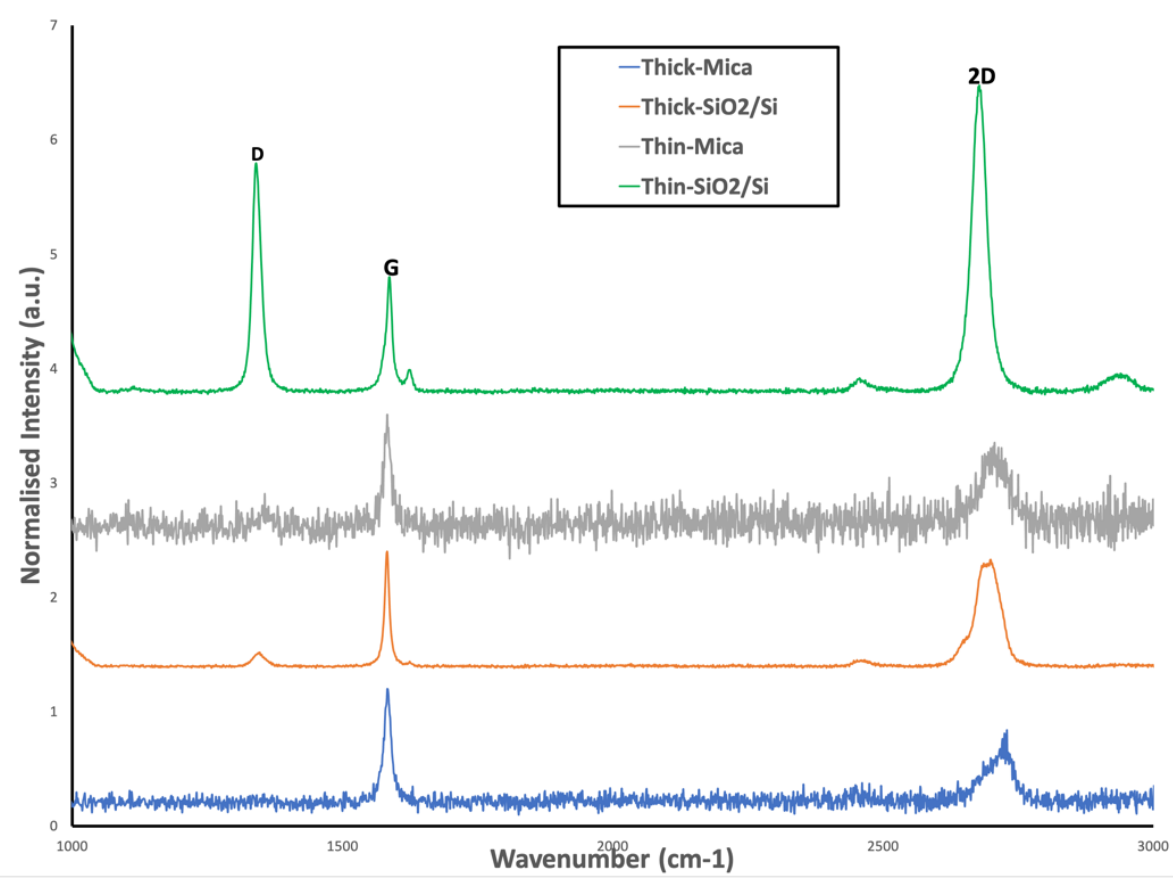

Figure 5.2: Raman spectra (normalised by the G-band intensity.)of pre-annealing 'thick' S1 and M1 and 'thin' S2 and M2 regions of the graphene attached to the $\mathrm{SiO}_{2} / \mathrm{Si}$ and mica substrates.

The $\mathrm{I}_{2 \mathrm{D}} / \mathrm{I}_{\mathrm{G}}$ ratio is often the first measure used to assess the number of layers. In Figure 5.2 , in the top spectrum for $\mathrm{S} 2$ (thin graphene on $\mathrm{SiO}_{2}$ ), $\mathrm{I}_{2 \mathrm{D}} / \mathrm{I}_{\mathrm{G}}=2.6$, indicating singlelayer graphene. For $\mathrm{S} 1$ (thick graphene on $\mathrm{SiO}_{2}$ ), $\mathrm{I}_{2 \mathrm{D}} / \mathrm{I}_{\mathrm{G}}=0.9$, indicating bi-layer graphene. In addition, the 2D-band shape for sample S2 has a very sharp peak, which indicates a single layer of graphene on $\mathrm{SiO}_{2} / \mathrm{Si}$, while sample $\mathrm{S} 1$ becomes broader, which indicates that $\mathrm{S} 1$ has thicker thickness than S2. An issue arises in the use of $\mathrm{I}_{2 \mathrm{D}} / \mathrm{I}_{\mathrm{G}}$ to assess the number of layers in mechanically exfoliated graphene, where, depending on the alignment of layers relative to each other, the 2D band can broaden, reducing the band intensity and breaking the $\mathrm{I}_{2 \mathrm{D}} / \mathrm{I}_{\mathrm{G}}$ relationship used to assess the number of layers [17]. The intensity ratio is 0.5 for M1 and 0.7 for M2, broadly indicating multi-layer and few-layer graphene, respectively. However, because both peaks have broadened, these are unlikely to be correct for mica.

To overcome this issue, the FWHM of the 2D band can used instead. For the samples on $\mathrm{SiO}_{2}$, in Figure 5.2, FWHM (G) is equal to $31 \mathrm{~cm}^{-1}$ for $\mathrm{S} 2$ and $52 \mathrm{~cm}^{-1}$ for $\mathrm{S} 1$, identifying them as single-layer and bi-layer, respectively, in direct agreement with the $\mathrm{I}_{2 \mathrm{D}} / \mathrm{I}_{\mathrm{G}}$ ratio. The FWHM of the $2 \mathrm{D}$ band for graphene on mica is characteristic of 
tri-layer for sample M1 and bi-layer for sample M2, at $59 \mathrm{~cm}^{-1}$ and $51 \mathrm{~cm}^{-1}$, respectively. Given the broadening of the $2 \mathrm{D}$ peaks in all but sample S2, these are the most reliable measures of layer number.

The final way to assess the layer number is to use the positions of the $\mathrm{G}$ band and the 2D band. The G-band frequency downshifts and the 2D-band upshifts with increasing $\mathrm{n}$ (for small n) [14,16]. Addressing just the mica samples, where there is not yet consensus, the position of the $\mathrm{G}$ band for sample M2 (thin) is $1,583 \mathrm{~cm}^{-1}$, while it is $1,580 \mathrm{~cm}^{-1}$ for thick graphene (M1). The position of the 2D band is $2,692 \mathrm{~cm}^{-1}$ for M2 and 2,727 $\mathrm{cm}^{-1}$ for M1, which has shifted to a higher frequency. These positions do not give a quantitative assessment of layer number, but do confirm that the peak positions agree that M2 is thinner than M1, which was already apparent from the electron microscopy intensity. However, this backs up the use of the 2D-band shape and FWHM as the primary measure of the layer number, given above. For these four samples, this Raman analysis then gives layer numbers of: $\mathrm{S} 1=2$ (bi-layer graphene), $\mathrm{S} 2=1$ (single-layer graphene), M1 = 3 (tri-layer graphene) and M2 = 2 (bi-layer graphene).

The topological profiles of the graphene sheets for these four samples were also measured by AFM, as shown later in figures 5.7 and 5.8, where they are compared to post-annealing profiles. Looking ahead, it will be seen that comparing the height of the graphene stack to the average height of the surrounding substrate, this gives the graphene thickness with $\operatorname{AFM}(\mathrm{S} 1 \approx 3 \mathrm{~nm}, \mathrm{~S} 2 \approx 2 \mathrm{~nm}, \mathrm{M} 1 \approx 6 \mathrm{~nm}$ and $\mathrm{M} 2 \approx 1 \mathrm{~nm})$. It is reported in the literature that the height of single-layer graphene measured with AFM in ambient conditions can range from 0.4 to $1.7 \mathrm{~nm}$. Sample S2 (single-layer) falls within this range as expected, but so too does M2, which is determined with Raman analysis to be bi-layer. AFM is widely regarded to be an inaccurate and unreliable method for layer-number determination, particularly for single-layer graphene. The difference is generally considered to be related to variation in interactions such as substrate-graphene and AFM probe-graphene bonding, which depend upon graphene structure, substrate surface energy and preparation of sample [18]. So, bringing together the Raman spectroscopy and AFM results, it is concluded that the Raman result is the most reliable technique for determining the number of layers which are S1(bilayer),S2 (single layer), M1 (tri-layer) and M2 (bi-layer), but 
that AFM is useful for showing more details about the morphology of graphene sheets and tracking the changes that occur after annealing, which will be addressed later.

Next, the defect or disorder in the four samples before annealing is addressed. The Dband intensity is usually used to indicate the quality of graphene. As shown in Figure 5.2 , the D peak is nearly absent in both thick and thin graphene on mica (samples M1 and M2), which indicates defect-free graphene. On the other hand, the D band is clearly present in both sheets of graphene on $\mathrm{SiO}_{2}$ (samples $\mathrm{S} 1$ and $\mathrm{S} 2$ ). The ratio of peak intensities $\mathrm{I}_{\mathrm{D}} / \mathrm{I}_{\mathrm{G}}$ for the thinner sheet (S2) is 2, much higher than that of the thicker sheet (S1), which is $0.12 \mathrm{~nm}$; this indicates that the structural defect or disorder is higher in the thinner sheet of graphene (Figure 5.3). It has been stated that the intensity of the D peak reduces significantly as the number of layers of graphene increases $[10,16]$. This is possibly due to thick layers having higher rigidity and consequently being less likely to conform to the substrate during deposition [19]. Overall, the absence of a D peak in graphene on mica proves it to be flat graphene, while graphene on $\mathrm{SiO}_{2}$ is not defect-free. However, the changes in $\mathrm{D}$ band for the four samples will be investigated more in the next section.

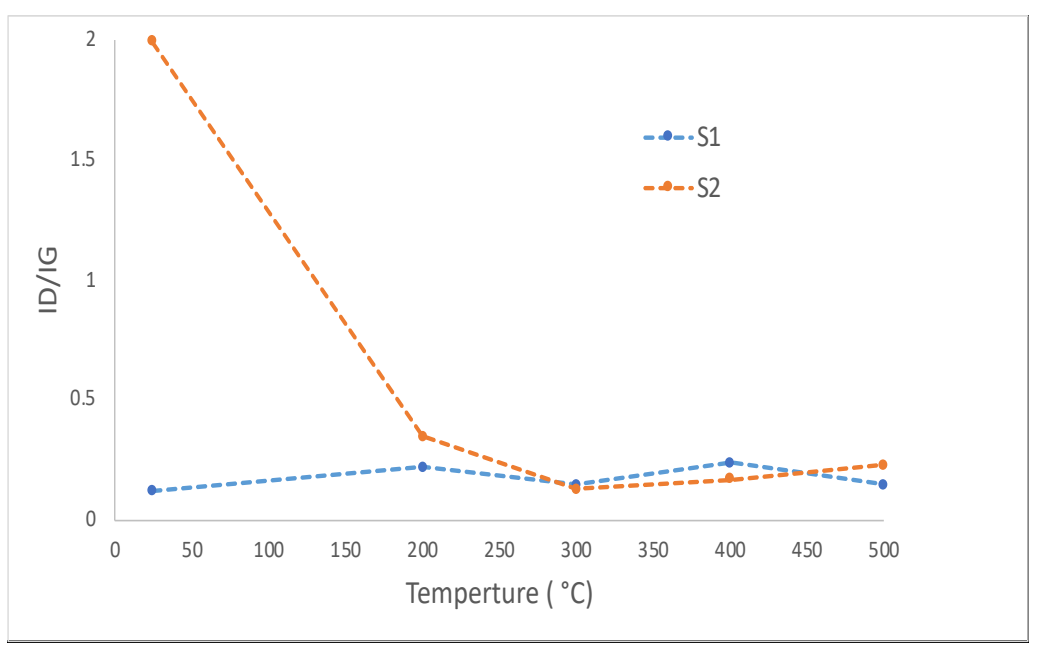

Figure 5.3: $\mathrm{I}_{\mathrm{D}} / \mathrm{I}_{\mathrm{G}}$ ratio of 'thick' $(\mathrm{S} 1)$ and 'thin' $(\mathrm{S} 2)$ graphene on $\mathrm{SiO}_{2}$. Dashed lines guide the eye.

\subsubsection{Post-annealing stage}

With the initial parameters determined, the changes that occur during annealing will be examined. The four sample areas S1, S2, M1 and M2 were annealed in a UHV at temperatures of $200^{\circ} \mathrm{C}, 300^{\circ} \mathrm{C}, 400^{\circ} \mathrm{C}$ and $500^{\circ} \mathrm{C}$. Looking first at the effects on the presence of defects, from Figure 5.4 ( $a$ and $b$ ), the D peak is visible in the Raman 
spectra for the samples of thick (S1) and thin (S2) graphene on $\mathrm{SiO}_{2}$. However, the thicker sheet (S1) shows lower $\mathrm{I}_{\mathrm{D}}$ than the thinner sheet before annealing, as mentioned above. All spectra are normalised to the G-band intensity to remove any change from focusing or alignment issues. The normalised $\mathrm{I}_{\mathrm{D}} / \mathrm{I}_{\mathrm{G}}$ ratio is widely used in order to measure the amount of disorder. The $\mathrm{I}_{\mathrm{D}} / \mathrm{I}_{\mathrm{G}}$ ratio is extracted and plotted against temperature for S1 and S2 in Figure 5.3. For both S1 and S2, the defect before annealing has reduced after annealing at low temperature, then started to increase at high temperature. The thinner sample (S2) shows a continual reduction in defect from pre-annealing to low temperature, then increases at $400^{\circ} \mathrm{C}$ (high temperature). The lowest number of defects is found at a temperature of $300^{\circ} \mathrm{C}$.

Returning to the Raman spectra, Figure 5.4 (c and d) shows the Raman spectra of annealed graphene on mica for the thicker (M1) and thinner (M2) samples. No defect peak is evident for M1. For M2 there may be a slight defect peak, but it is not sufficiently out of the noise floor to be detected. Although the collection settings are the same for all four sample areas, the level of noise in the graphene on the mica samples is higher (and highest with the thin graphene on mica), due to the transparency of mica substrate, as thinner layers have higher transmittance, while the thick layers have more interaction with the incident light. It should be noted that some annealing processes are known to induce defect peaks in graphene, so it is important to check that disorder does not occur after annealing, which the Raman spectra in Figure 5.4 (c) confirm.

In these indicative samples, and across all other samples of graphene on mica prepared for this work, no defect peak was ever detected in Raman analysis, in stark contrast to as-prepared graphene on $\mathrm{SiO}_{2}$ samples. As seen with other work, the defects in graphene on $\mathrm{SiO}_{2}$ increase with annealing, but here no formation or increase of defects was seen on mica. This is an important finding, which will be discussed in detail in the conclusion of this chapter, once strain has been considered. 

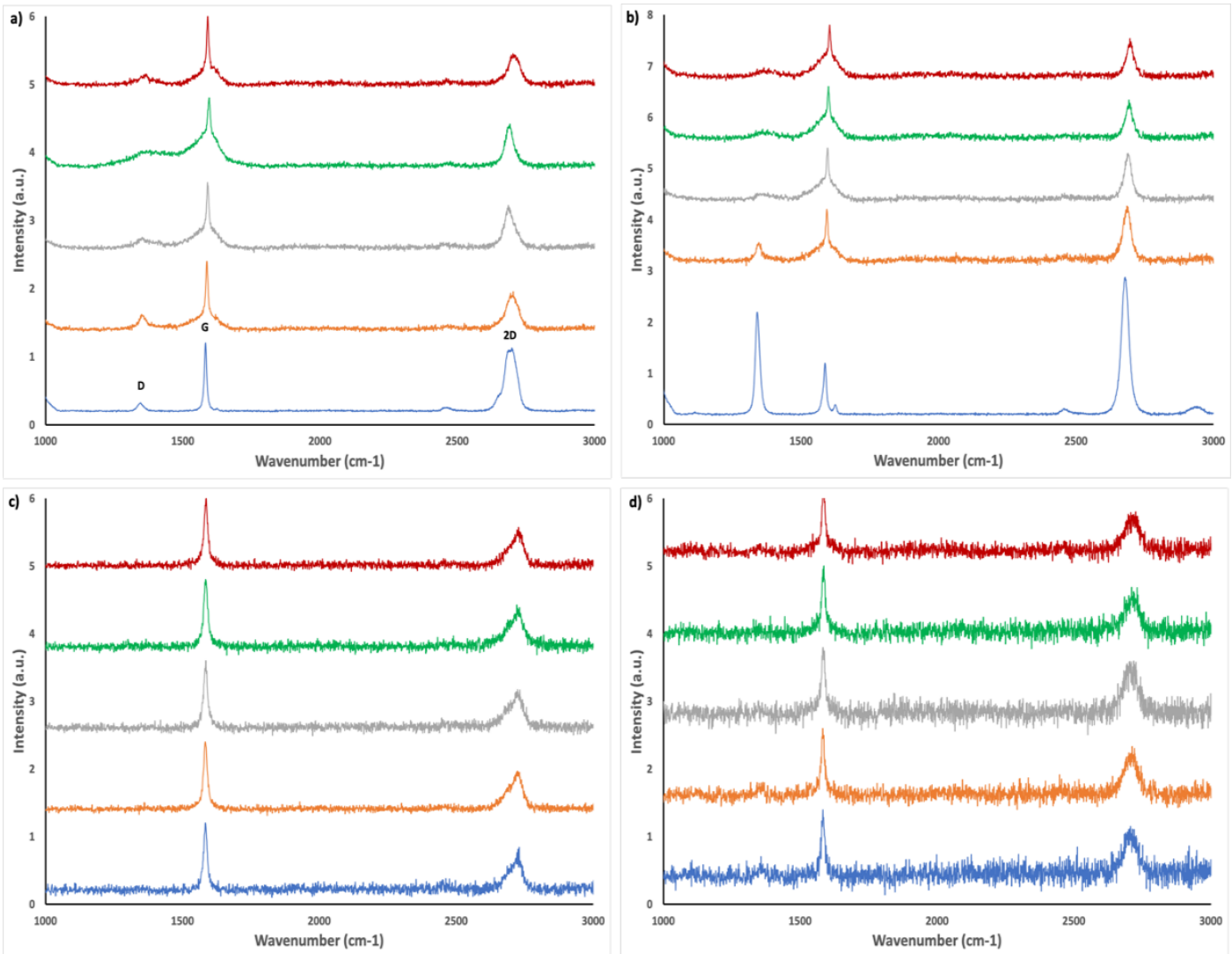

Figure 5.4: Raman spectra of samples (a) S1 and (b) S2 (graphene on $\mathrm{SiO}_{2} / \mathrm{Si}$ ), and (c) M1 and (d) M2 (graphene on mica). Spectra (normalised by the G-band intensity ) from bottom to top, pre-annealing and postannealing, at $200^{\circ} \mathrm{C}, 300^{\circ} \mathrm{C}, 400^{\circ} \mathrm{C}$ and $500^{\circ} \mathrm{C}$, respectively.

Another method of assessing disorder in the deposited graphene is through changes in the $\mathrm{G}$ and 2D bands, including their positions and FWHM, which are caused either by strain or doping. The start and change in each peak position are given in Table 5.1 and then plotted against temperature in Figure 5.5, while the bandwidth of these bands is shown in Figure 5.6. 


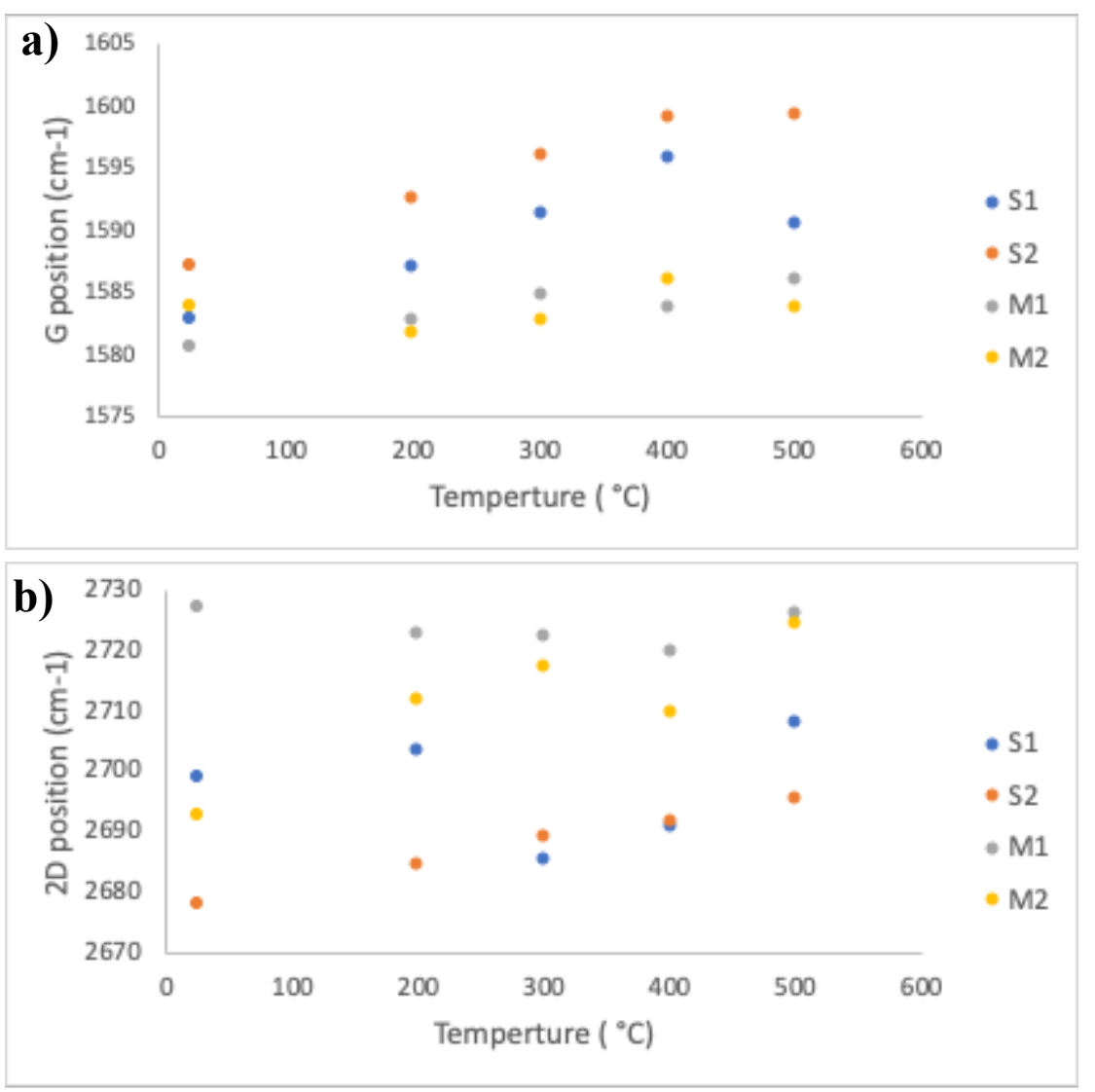

Figure 5.5: Position of a) G and b) 2D bands for thick graphene (S1 and M1) and thin graphene (S2 and M2) at different annealing temperatures.

First, the positions of the $\mathrm{G}$ and $2 \mathrm{D}$ bands against temperature will be considered. The initial 2D- and G-band positions show a distribution, because these are affected by the number of graphene layers, as explored earlier. Samples S1 and M2 have nearly the same position, because both are bi-layer. Changes in strain during annealing can shift these initial positions. As shown in Figure 5.5 (a), the change in the G-band position for the mica samples is not consistent, although there may be an increasing trend for M1. This is in contrast to both silicon samples, where there is a clear trend of increasing G-band position during annealing. Figure 5.5 (b) shows the 2D-band position for all samples. Here, the mica results are again mixed, with M2 broadly increasing and M1 reducing, except for the transition from $400^{\circ} \mathrm{C}$ to $500^{\circ} \mathrm{C}$. The silicon sample $\mathrm{S} 2$ shows a nearly linear increase with temperature, and $\mathrm{S} 1$ also shows an increase, if $300^{\circ} \mathrm{C}$ and $400^{\circ} \mathrm{C}$ are omitted. These changes are addressed together, later in this paper.

The next feature is the FWHM of the G band, plotted against temperature in Figure 5.6 (a). Again, there is no clear trend for the mica samples, but comparing $400^{\circ} \mathrm{C}$ and 
$500^{\circ} \mathrm{C}$ to the start position, the FWHM increases for both of the silicon samples. For the FWHM of the 2D band, shown in Figure 5.6 (b), there is one notably global trend: at $400^{\circ} \mathrm{C}$ all values drop from the initial value, and then all values but that for M1 increase again at $500^{\circ} \mathrm{C}$.
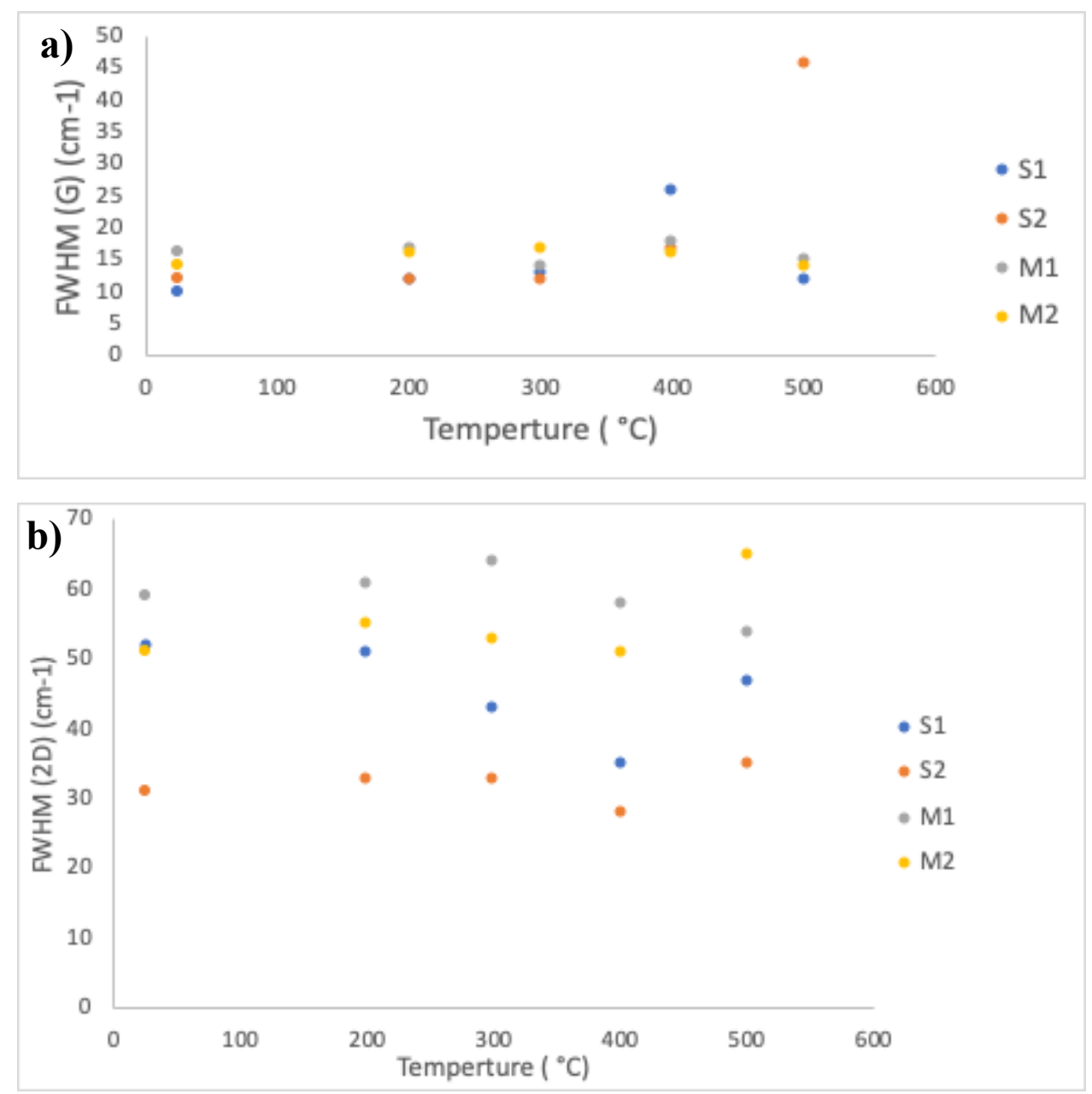

Figure 5.6: FWHM of a) G and b) 2D bands for thick ( $\mathrm{S} 1$ and M1) and thin (S2 and M2) graphene at different annealing temperatures.

Many studies show that the change in the position and FHWM of G and 2D bands can be caused by both strain [20-24] and doping [25-30], and so both causes must be assessed.

First, the magnitude of the shifts in comparison to each other is important. If the blue shift of $\mathrm{G}$ and the blue shift of 2D are broadly similar, it may indicate that thermal annealing has induced hole doping in the graphene $[25,26]$. However, when the upshift of the $2 \mathrm{D}$ band $\left(\Delta \omega_{2} \mathrm{D}\right)$ is not similar to the upshift of the $\mathrm{G}$ band $\left(\Delta \omega_{\mathrm{G}}\right)$, it could mean 
that annealing has caused a strain in the graphene [20]. For instance, the blue shift of the $2 \mathrm{D}$ band $\left(\Delta \omega_{2} \mathrm{D}\right)$ has been noted to be $\sim 2$ times greater than the G-band upshift $\left(\Delta \omega_{\mathrm{G}}\right)$; this was found to be strain [20]. In the results of the present study, after annealing at $500^{\circ} \mathrm{C}$ (as shown in Table 5.1), the blue shift of the $\mathrm{G}$ band $\left(\Delta \omega_{\mathrm{G}}\right)$ for $\mathrm{S} 2$ is $12 \mathrm{~cm}^{-1}$, while the blue shift of the $2 \mathrm{D}$ band $\left(\Delta \omega_{2} \mathrm{D}\right)$ is $17 \mathrm{~cm}^{-1}$. In addition, $\mathrm{S} 1$ shows blue shifts of $7.7 \mathrm{~cm}^{-1}$ and $3.6 \mathrm{~cm}^{-1}$, for $\mathrm{G}$ and $2 \mathrm{D}$ bands, respectively. For $\mathrm{SiO}_{2}$, both samples show dissimilar blue shifts for both bands, and so strain is concluded as the cause. Neither mica sample follows the established pattern for either strain or hole doping.

Table 5.1: Values of position of $G$ band before annealing of graphene sheets and change in frequency of $\mathrm{G}$ band after annealing at $500^{\circ} \mathrm{C}$, for thin and thick graphene on $\mathrm{SiO}_{2}$ and mica substrates.

\begin{tabular}{|c|c|c|c|c|}
\hline Thickness & Thin layer & & Thick la & \\
\hline Substrate & $\begin{array}{l}\mathrm{Sio}_{2} / \mathrm{Si} \\
(\mathrm{S} 2)\end{array}$ & $\begin{array}{l}\text { Mica } \\
\text { (M2) }\end{array}$ & $\begin{array}{l}\mathrm{Sio}_{2} / \mathrm{Si} \\
(\mathrm{S} 1)\end{array}$ & $\begin{array}{l}\text { Mica } \\
\text { (M1) }\end{array}$ \\
\hline $\begin{array}{l}\text { Position of } G \\
\text { band } \\
\left(\mathrm{cm}^{-1}\right)\end{array}$ & 1587.05 & 1583.78 & 1582.66 & 1580.49 \\
\hline $\begin{array}{l}\Delta \omega_{G} \\
\left(\mathrm{~cm}^{-1}\right)\end{array}$ & 12.13 & -0.1 & 7.7 & 5.39 \\
\hline $\begin{array}{l}\text { Position of } 2 \mathrm{D} \\
\text { band } \\
\left(\mathrm{cm}^{-1}\right)\end{array}$ & 2678.03 & 2692.69 & 2699.07 & 2727.33 \\
\hline $\begin{array}{l}\Delta \omega_{2 D} \\
\left(\mathbf{c m}^{-1}\right)\end{array}$ & 17.45 & 31.84 & 3.67 & -0.98 \\
\hline
\end{tabular}

Second, both hole and electron doping can lead to a blue shift of the $\mathrm{G}$ band and reduce its bandwidth [27-30]. It is stated that graphene is strongly hole-doped after vacuum annealing when the $\mathrm{G}$ and 2D bands upshift, and the FWHM of G band reduces [26]. Furthermore, a blue shift in the G band coupled with a decreasing FWHM has been attributed to both electron and hole doping [27]. These studies indicate that the shift of $\mathrm{G}$ and $2 \mathrm{D}$ bands with reduction in FWHM for $\mathrm{G}$ band is a sign of a doping. In 
contrast, if the $\mathrm{G}$ band upshifts but the FWHW does not change or increases, this indicates strain [20]. Comparing this with the results here after annealing at $500^{\circ} \mathrm{C}$, for the $\mathrm{SiO}_{2}$ samples there is consistent increase in both the position and the FWHM of the $\mathrm{G}$ band, consistent with increasing strain during annealing. For mica samples, after annealing at $500^{\circ} \mathrm{C}$ the results are not clear .For example, for sample M1 there is a blue shift in G band with reduction in its FWHM, possibly implying doping, but also a slight red shift in $2 \mathrm{D}$ band, whereas, for sample $\mathrm{M} 2$, there is a decrease in the bandwidth of $G$ but also a slight red shift in the $G$ band; thus the above literature may not apply to these samples. However, a study of annealed chemical vapour deposition (CVD) graphene on mica has attributed the G-band downshift and broadened FWHM to decreased poly (methyl methacrylate) (PMMA) doping (used in their transfer method), and the 2D-band upshift to band structure modification [31]. Another study states that the absence of electron-doped graphene/mica samples is not consistent with an inhomogeneous distribution of $\mathrm{K}$ atoms [32].

Third, strain induces significant upshift of the 2D band, according to the literature [20, 22]. This is in agreement with the graphene on $\mathrm{SiO}_{2}$ samples here. However, 2D increased at $500^{\circ} \mathrm{C}$ for mica substrate $\mathrm{M} 2$, but reduced for $\mathrm{M} 1$, while the G-band position saw no significant change in either. This analysis is therefore not appropriate here; this is discussed later in the paper.

Bringing the analysis of all the Raman results together, first, a change in doping during annealing can be ruled out, as the changes seen are not consistent with substrateinduced hole or electron doping. Samples are annealed in UHV, so there can be no oxygen doping during annealing. However, it has been observed that vacuum annealing can make graphene more active and increase the molecular adsorption once exposed to air, leading to subsequent doping from $\mathrm{H}_{2} \mathrm{O}$ and $\mathrm{O}_{2}$ [26]. Although samples here were removed from vacuum for Raman analysis, no induced effects from any of these dopants are observed.

For $\mathrm{SiO}_{2}$, the upshift of the $\mathrm{G}$ and 2D bands, coupled with an FWHM of the $\mathrm{G}$ band that either increases or remains constant, is a clear indicator of strain. On mica, the picture is less consistent, and some expected indicators of increasing strain are absent. This is not surprising, because the first-order D band, which is primarily used to indicate strain in the as-deposited samples, is missing or undetectable. With no 
detectable strain in the starting condition, there are less likely to be changes in strain during annealing. No added strain is detected during annealing.

Here, the annealing impact on graphene strain that caused Raman shift of G and 2D bands is discussed. There are two factors that lead to Raman shift: temperature of annealing and symmetry of molecules. In particular, the thermal expansion coefficient (TEC) of materials involved in solid interfaces could be a parameter characterising the stress within the materials, which in turn can modify their electronic properties.

It is well known that in experiments where graphene is supported on a substrate, the graphene structure is sensitive to the nature of the support. When the two materials have different thermal expansion coefficients, this can lead to strain during heating. Graphite and graphene have negative thermal expansion coefficients while $\mathrm{SiO}_{2}$ and mica substrates have positive thermal expansion coefficients. The heat treatment therefore causes a change in the graphene layer stress as a result of the contrasting signs of the thermal expansion coefficients of graphene and the substrate; both substrates increase in length with temperature rises, whereas the graphene sheets contract. After cooling, the opposite occurs, i.e. the substrate contracts and the graphene expands, which leads to compressive strain in the graphene.

The number of graphene layers plays a role in the interaction with the substrate. As discussed above, this mismatch of the TEC induces tensile strain in the graphene during heating and compressive strain on cooling. The van der Waals interactions, which fix the graphene to the substrate, differ for thicker and thinner graphene stacks. In the case of thin graphene, the interaction that holds the graphene to the substrate is greater, and is affected more by the substrate than in the case of the thick sheet. Therefore, the mismatch in TEC has a significant impact on thinner sheets, with effects on their properties such as buckling or slipping. It is observed that slips happened in single-layer and bi-layer graphene, with a blueshift of the $\mathrm{G}$ band noted over $400 \mathrm{~K}$. However, a smaller shift of $G$ band means the slip is not observed in the case of graphite [33]. If the van der Waals interaction between graphene and the substrate is weak, the strain between them will not be coherent, producing less slippage $[19,33,34]$. After cooling, there is compressive strain, which causes mechanical distortions of graphene such as the formation of ripples $[33,35]$. However, if the annealing occurs at a low temperature, the tensile strain is sufficiently small during annealing and, after 
cooling, the sheet returns to its original state without causing any compressive strain, as slippage starts to be active after annealing at $100^{\circ} \mathrm{C}$ or more $[33,34]$.

Comparing the above literature with the results of this study, the Raman shift of both the $2 \mathrm{D}$ and $\mathrm{G}$ peaks after $500^{\circ} \mathrm{C}$ is more for $\mathrm{S} 2$ than for $\mathrm{S} 1$, as shown in Table 5.1. This indicates more strain in the thinner sample (S2), consistent with the established annealing mechanism. The thin graphene (S2) shows a near-linear consistent increase in Raman shift with temperature, shown in Figure 5.5. The shift in the thicker sample (S1) drops back at $300^{\circ} \mathrm{C}$ and $400^{\circ} \mathrm{C}$, but continues to increase at $500^{\circ} \mathrm{C}$. This is an important observation, confirming that thinner sheets are more sensitive to strain after annealing. For mica samples, the pattern of strain is not as clear; this is discussed later. The final method to assess the graphene changes during annealing is AFM, which was carried out before and after the final annealing steps. Figure 5.7 (a) shows graphene sheets on thick (S1) and thin (S2) $\mathrm{SiO}_{2}$ layers before annealing. Both are plotted on the same z-axis colour scale for comparison. The upper portion of the flake is singlelayer, with the line profile S2b showing a thickness of $2 \mathrm{~nm}$. The mottled region at the bottom of the same flake is the folded-over double-height section. This is clearer in the SEM image (shown in Figure 5.1); it was harder to image in AFM, although AFM does uniquely give the required topographic information. Line profile S2b shows the height of the thin section to be $2 \mathrm{~nm}$. Line profile $\mathrm{S} 1 \mathrm{~b}$ is taken down through the sample to include the single layer, because the roughness of the thicker portion was too high to accurately determine the layer number against the background. Taking the line scan in this direction shows the thinner section is $3 \mathrm{~nm}$ high compared to the background, consistent with the horizontal line scan S2b, and also shows the rough thicker region. 

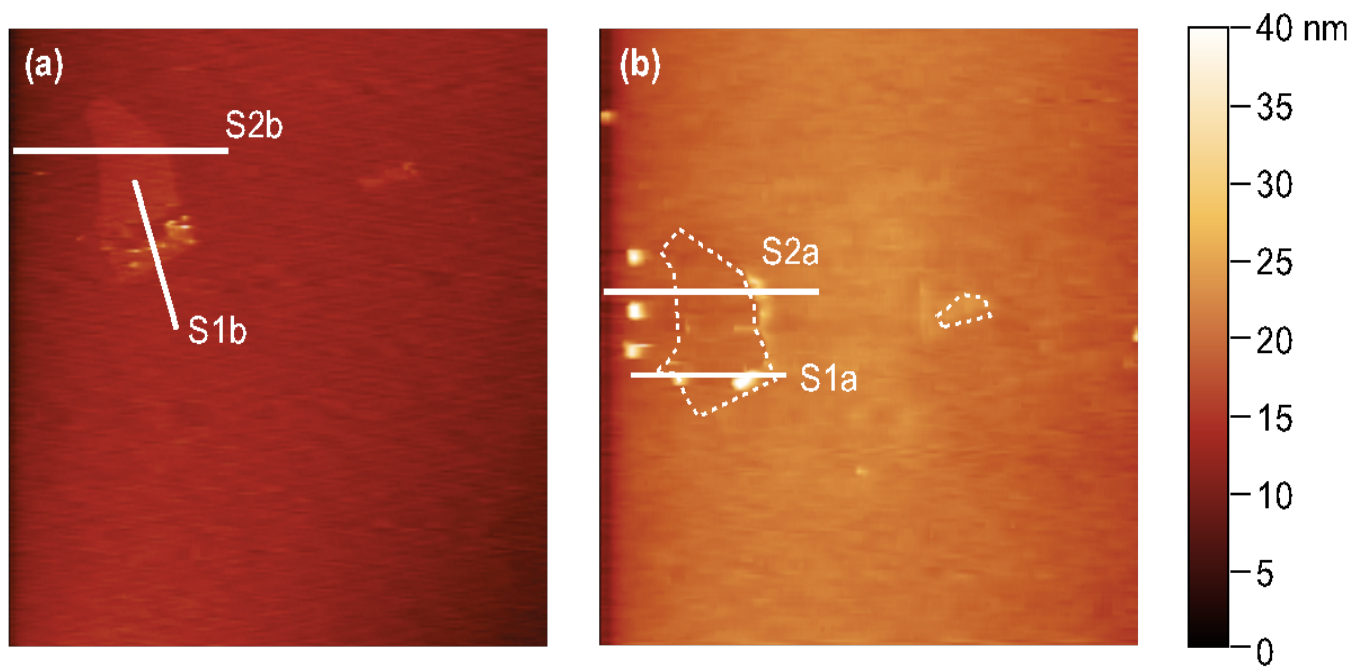

(c) S1b

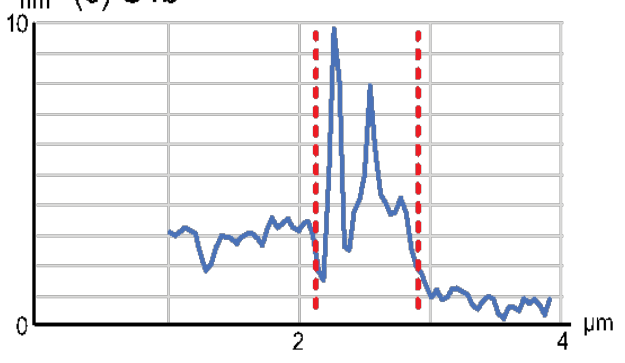

(d) S1a

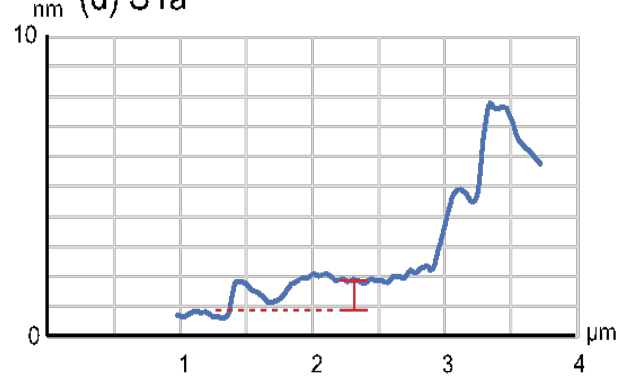

(e) $S 2 b$
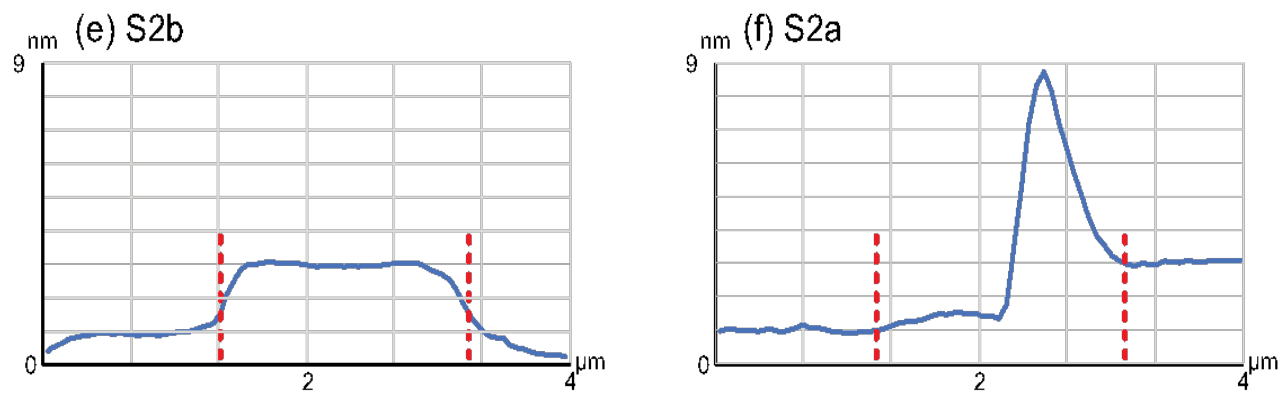

Figure 5.7: AFM images of (a) Sample S1 and S2 for graphene on $\mathrm{SiO}_{2}$ before annealing and b) after annealing at $500^{\circ} \mathrm{C}$.

The right-hand side of Figure 5.7 shows the same samples after annealing at $500^{\circ} \mathrm{C}$. The main flake is no longer visible in AFM, but the thicker flake (on the right) is. An outline of the flake and smaller piece were therefore taken from SEM and overlaid here as dashed white lines, using the additional piece of graphene to the right for alignment. The corresponding horizontal line scans underneath are averages of the three lines, to reduce noise. For the thicker S1a region, the noise has reduced and the folded sheet is now measured to be $\sim 1 \mathrm{~nm}$, indicated by the vertical red bar. In the line scan S2a, the extent of the flake is shown between the two vertical red dashed lines. The right-hand side of the graphene sheet appears to have bent upwards now, giving a 
white feature along the right edge and showing in the line scan as a large peak $8 \mathrm{~nm}$ above the left-hand-side background. Before this lifted side, the left side of the sample now shows a reduced height of $\sim 1 / 2 \mathrm{~nm}$.

Comparing the heights of S1and S2 after annealing, Figure 5.7 (c) shows the thickness of thick graphene before annealing, with height around $3 \mathrm{~nm}$, although rough, while this height is reduced after annealing to around $1 \mathrm{~nm}$, as shown in Figure 5.7 (d). The same observation was made for the thin sheet (S2), with a thickness of $2 \mathrm{~nm}$ shown in Figure 5.7 (e) there is a reduction in height after annealing at $500^{\circ} \mathrm{C}$ as well shown in Figure 5.7 (f). However, SEM is not helpful to illustrate this sample, as AFM provides better morphology and height difference information. This study therefore relies on AFM to demonstrate any change after the annealing process.

It is reported in the literature that the height of single-layer graphene measured with AFM in ambient conditions can range from 0.4 to $1.7 \mathrm{~nm}$. Sample S2 (single-layer) falls within this range as expected, but so too does M2, which is determined with Raman analysis to be bi-layer.

After annealing at $500^{\circ} \mathrm{C}$, the flake of graphene appears to have disappeared in the AFM image. This effect is attributed in the literature to a decrease in the number of graphene layers, as they are blown off or absorbed into the substrate after annealing [20]. However, the Raman result here shows that the sheets were still present, but no longer immediately visible under AFM. However, by aligning from the thicker fragment and taking averaged line profiles, we show the sheets can still be detected with AFM. The reduction in the height of the graphene is now comparable to the background roughness of the $\mathrm{SiO}_{2}$ and there is not sufficient contrast for it to be clearly detectable without averaging several line scans together.

The same presentation is shown for the mica substrate samples, in Figure 5.8. Here there are two distinct flakes of graphene: M2 is bi-layered and M1 is tri-layered. Figure 5.8 shows AFM image of samples M1 and M2 a) before and b) after annealing at $500^{\circ} \mathrm{C}$. This area is also shown in the SEM image in Figure 5.1. The left flake (M2) is bi-layered, with the line profile M2b showing a thickness before annealing of $\approx 1 \mathrm{~nm}$, as seen in the dashed vertical lines of the line profile. The right flake (M1) is trilayered, with the line profile M1b showing the height of the thick section to be $\approx 6 \mathrm{~nm}$. 
Comparing this height with the literature, it is always important to report the expected height of single-layer graphene, which then establishes the offset between the graphene and the substrate. Once this is known, each additional layer is assumed to be separated with an additional $0.335 \mathrm{~nm}$ for each layer of graphene. It has been found that, for single-layer graphene on mica, the height varies from 0.4 to $0.9 \mathrm{~nm}[18,36]$. This puts the height of $\mathrm{M} 2$, at $\sim 1 \mathrm{~nm}$, in line with the expected range for bi-layer graphene, consistent with the Raman results.
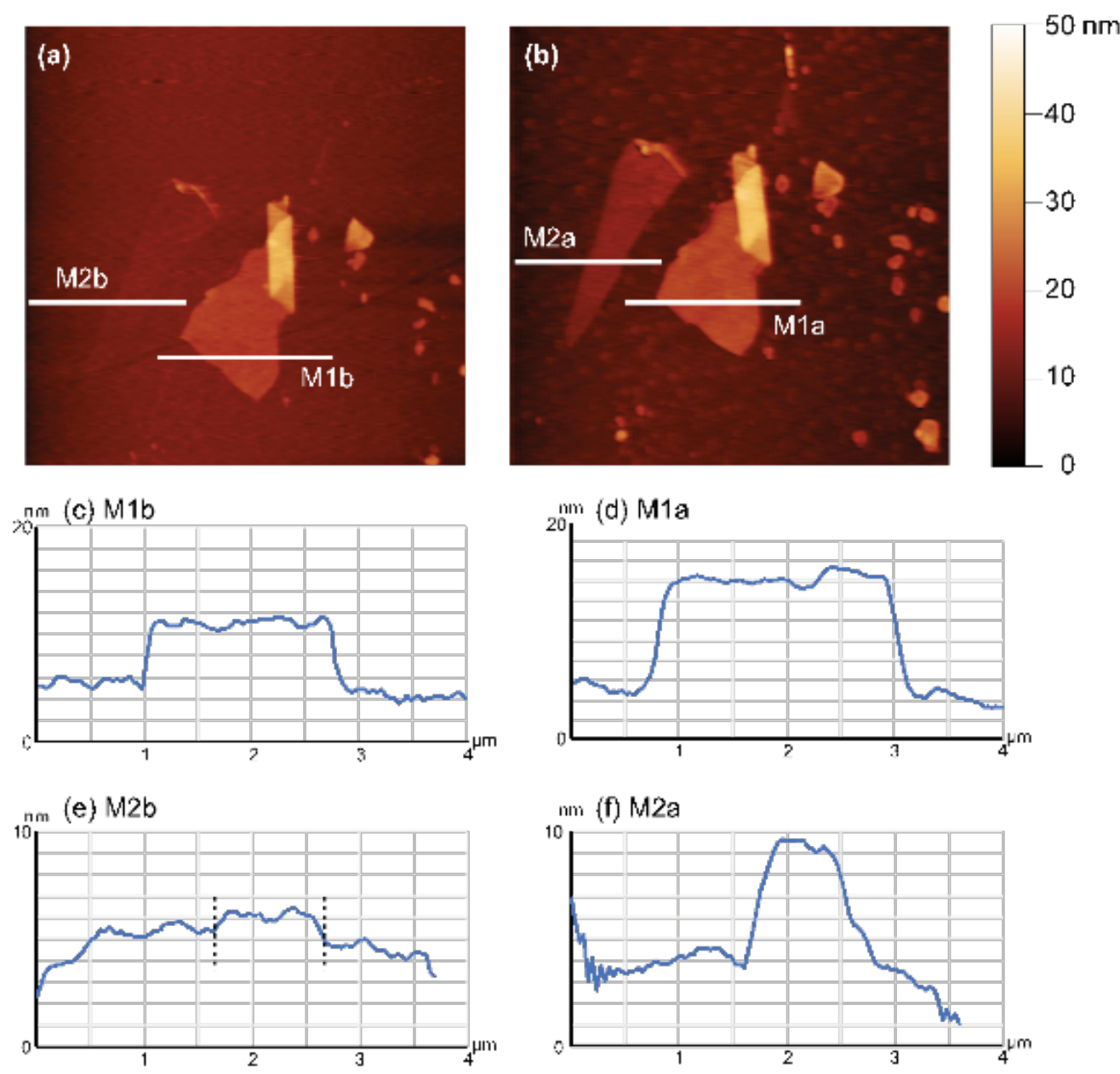

Figure 5.8: AFM images of (a) Sample M1 and M2 for graphene on mica before annealing and b) after annealing at $500^{\circ} \mathrm{C}$. It is important to mention that the same settings and mode of AFM were used before annealing and afterwards. 
For the thicker M1 sample graphene on mica, the picture is not as clear. M1 was determined by Raman analysis to be tri-layered. Using an upper estimate for singlelayer graphene thickness of $1 \mathrm{~nm}$ and an interlayer spacing of $0.335 \mathrm{~nm}$, the expected height of tri-layer graphene would be $1.67 \mathrm{~nm}$, whereas here it was measured to be $\sim 6$ $\mathrm{nm}$. Water trapped between graphene and mica has been measured to be in the range 0.3-1.5 nm, depending on AFM scanning parameters [32]. It is found that this layer of confined water between exfoliated graphene and mica increases the AFM step heights in ambient conditions. Water could be a part of the cause, but cannot totally explain the discrepancy. Instead, it is most likely that the step defect running diagonally under M1, faintly visible as a darker stripe in the figure before annealing, has increased or altered the determination of the height. AFM is known to be less reliable for such measurements, whereas Raman spectroscopy has proven to be the most reliable method of choice for determining the number of graphene layers [18]. Here it is assumed that the Raman spectroscopy determination is accurate.

Returning to Figure 5.8, the right-hand side shows the same samples M1 and M2 after annealing at $500^{\circ} \mathrm{C}$. The line profile $\mathrm{M} 2 \mathrm{a}$ shows a thickness of $\approx 6 \mathrm{~nm}$, whereas the line profile M1a shows a height of $\approx 11 \mathrm{~nm}$. Notable observations are the apparent increase in the graphene thickness after annealing and the appearance of dots across the whole of the substrate where there is no graphene. Before annealing, M1 had a height of $6 \mathrm{~nm}$, which increased to $11 \mathrm{~nm}$ after annealing at $500^{\circ} \mathrm{C}$; the thin sheet (M2) had an initial thickness of $1 \mathrm{~nm}$, which increased to $6 \mathrm{~nm}$. The increase in the apparent height of the graphene after annealing is an abnormal feature that has not been detected before; this work is its first observation. It is not possible to track the change in the number of graphene layers using Raman analysis, as the samples have been annealed and the Raman bands have shifted. However, AFM shows no apparent change in the shape of the flat or folded regions of either sample. There appears to be no conformational or structural deviation post annealing. It is most likely that the mica has been blown off around the graphene, with the graphene acting almost as a mask. This is supported by the disappearance of the step defect, which is no longer visible post annealing. 


\subsection{Conclusion}

Thermal annealing is a required step in producing materials and devices from graphene. However, the temperature, graphene thickness and substrate type all affect how the graphene responds chemically and structurally to the elevated temperature. This Chapter directly compares the annealing of few-layer graphene on $\mathrm{SiO}_{2}$ and mica substrates for the first time, using SEM, Raman and AFM, to monitor the disorder, electronic and mechanical properties.

Four representative samples areas are identified by AFM and SEM: bi-layer and single layer graphene on $\mathrm{SiO}_{2}$ and tri-layer and bi-layer graphene on mica. Before annealing, Raman showed disorder present in $\mathrm{SiO}_{2}$, but the characteristic Raman D-peak was absent for both mica samples presented, and all mica samples studied in this work. This is significant, because it confirms the proposition of this thesis that mica is a more suitable substrate for the investigation of $2 \mathrm{D}$ materials than the rougher $\mathrm{SiO}_{2}$, which this confirms. The D peak is observed much more clearly in the thin layer on $\mathrm{SiO}_{2}$, attributed to the thick layer having higher rigidity and therefore better resisting conformation to the rougher substrate which induces the disorder.

Samples were annealed in UHV at $200{ }^{\circ} \mathrm{C}, 300^{\circ} \mathrm{C}, 400{ }^{\circ} \mathrm{C}$ and $500{ }^{\circ} \mathrm{C}$, with Raman conducted after each annealing step and AFM before and after the final step. The disorder or defect peak in $\mathrm{SiO}_{2}$ reduced at lower temperature anneals up to $300{ }^{\circ} \mathrm{C}$, but from $400{ }^{\circ} \mathrm{C}$ onwards increased.

An increase in the strain of graphene samples on $\mathrm{SiO}_{2}$ is observed, confirmed by the increase in width and the position of the $\mathrm{G}$ and 2D bands. Mismatch in the thermal expansion coefficients leads to compressive strain when graphene is annealed at high temperatures, to which the changes in strain are attributed. This strain increased at the annealing temperature increased. This is consistent with ours and other's previous work.

Another interesting effect occurs after annealing both graphene samples on $\mathrm{SiO} 2$ at $500{ }^{\circ} \mathrm{C}$ - they appear to become 'invisible' in AFM. Remarkably this effect has only been reported once before, by this group, where four-layer graphene on $\mathrm{SiO} 2$ was observed to 'disappear' with SEM. [10] The reason no other groups have reported this is likely because the 'disappearance' of graphene after annealing is always attributed in the literature to a decrease in the number of graphene layers as they are blown off 
or absorbed into the substrate after annealing [20]. However, the Raman results of the present study show that the sheets were still present, but no longer visible under AFM. This study used a new approach and aligned the AFM scans to other extant features on the surface and averaged several line profiles over the 'disappeared' graphene. This method confirmed that the graphene was still present, albeit with a much lower apparent height than before annealing. Given that one sample was single layer graphene and shown to still be present by Raman and AFM, the theory that layers are desorbed during annealing is called in to question by this work. It is more likely that due to the roughness of $\mathrm{SiO}_{2}$, graphene has conformed to the underlying substrate topography as annealing induces compressive strain. This is important for all graphene on $\mathrm{SiO}_{2}$ studies.

Annealing graphene on mica at the same temperatures failed to bring out the D-peak characteristic of disorder or defects. This is also significant, confirming that high temperature annealing can clean the graphene without causing any conformation changes in the graphene itself. Possible doping affects were seen at $500{ }^{\circ} \mathrm{C}$, but these were not consistent.

An unexpected but remarkable effect occurred after annealing mica at $500^{\circ} \mathrm{C}$ : a random distribution of dots over the mica surface. Mica was expected to be stable during annealing up to $600{ }^{\circ} \mathrm{C}$ [36], but here the surface is clearly changing at lower temperatures with the appearance of randomly-distributed 'dots' which appear as $1 \mathrm{~nm}$ raised sections in AFM. The only comparable report found in the literature reported nano blisters forming in graphene on mica annealed for 19 hours at $600{ }^{\circ} \mathrm{C}$, but located underneath the graphene, rather than on the exposed mica [36]. Here, samples are only annealed for $1 \mathrm{~h}$ at $500{ }^{\circ} \mathrm{C}$ and the 'blisters' are clearly formed on the exposed mica. AFM does not appear to show any blisters under the graphene itself, and there is no change in the strain of the graphene sheets which would be expected. The most significant result is that the apparent height of the graphene changes when this effect occurs, which has not been reported before. Raman cannot confirm that the number of graphene layers remains stable post annealing, since the peaks move from other effects. However, the structure of the bi-layer and tri-layer graphene on mica appears identical with AFM post annealing. It appears that the graphene is 'masking' the mica surface, protecting the area it covers, while the surface around it is 'blown off' at lower temperatures and shorter annealing times than previously reported. 
AFM was only carried out after the final annealing at $500{ }^{\circ} \mathrm{C}$ and so it is not known exactly at which temperature this new effect occurs, or what any intermediate stages may involve. Following the results of this chapter, a new study was designed specifically to investigate this new mica degradation and masking effect, presented in the next chapter. 


\subsection{References}

[1] Gao W, Huang R. Effect of surface roughness on adhesion of graphene membranes. Journal of Physics D: Applied Physics. 2011;44(45): 452001.

[2] Bunch JS, Dunn ML. Adhesion mechanics of graphene membranes. Solid State Communications. 2012;152(15): 1359-1364.

[3] Deng S, Berry V. Wrinkled, rippled and crumpled graphene: An overview of formation mechanism, electronic properties, and applications. Materials Today. 2016;19(4): 197-212.

[4] Lui CH, Liu L, Mak KF, Flynn GW, Heinz TF. Ultraflat graphene. Nature. 2009;462(7271): 339-341.

[5] Goncher SJ, Zhao L, Pasupathy AN, Flynn GW. Substrate level control of the local doping in graphene. Nano Letters. 2013;13(4): 1386-1392.

[6] Yamamoto M, Einstein TL, Fuhrer MS, Cullen WG. Charge inhomogeneity determines oxidative reactivity of graphene on substrates. ACS Nano. 2012;6(9): 8335-8341.

[7] Lin YC, Lu CC, Yeh CH, Jin C, Suenaga K, Chiu PW. Graphene annealing: How clean can it be? Nano Letters. 2012;12(1): 414-419.

[8] Kumar K, Kim YS, Yang EH. The influence of thermal annealing to remove polymeric residue on the electronic doping and morphological characteristics of graphene. Carbon. 2013;65: 35-45.

[9] Hong J, Park MK, Lee EJ, Lee D, Hwang DS, Ryu S. Origin of new broad Raman D and G peaks in annealed graphene. Scientific Reports. 2013;3: 2700.

[10] Alyobi MM, Barnett CJ, Cobley RJ. Effects of thermal annealing on the properties of mechanically exfoliated suspended and on-substrate few-layer graphene. Crystals. 2017;7(11): 349 .

[11] Fang XY, Yu XX, Zheng HM, Jin HB, Wang L, Cao MS. Temperature- and thickness-dependent electrical conductivity of few-layer graphene and graphene nanosheets. Physics Letters A. 2015;379(37): 2245-2251. 
[12] Nirmalraj PN, Lutz T, Kumar S, Duesberg GS, Boland JJ. Nanoscale mapping of electrical resistivity and connectivity in graphene strips and networks. Nano Letters. 2011;11(1): 16-22.

[13] Novoselov KS, Geim AK, Morozov SV, Jiang D, Zhang Y, Dubonos SV, et al. Electric field effect in atomically thin carbon films. Science. 2004;306(5696): 666-669.

[14] Ferrari AC, Meyer JC, Scardaci V, Casiraghi C, Lazzeri M, Mauri F, et al. Raman spectrum of graphene and graphene layers. Physical Review Letters. 2006;97(18): 187401.

[15] Hao Y, Wang Y, Wang L, Ni Z, Wang Z, Wang R, et al. Probing layer number and stacking order of few-layer graphene by Raman spectroscopy. Small. 2010;6(2): 195-200.

[16] Gupta A, Chen G, Joshi P, Tadigadapa S, Eklund PC. Raman scattering from high-frequency phonons in supported n-graphene layer films. Nano Letters. 2006;6(12): 2667-2673.

[17] Wall M. The Raman spectroscopy of graphene and the determination of layer thickness. Thermo Fisher Scientific. Application Note: 52252, 2011.

[18] Shearer CJ, Slattery AD, Stapleton AJ, Shapter JG, Gibson CT. Accurate thickness measurement of graphene. Nanotechnology. 2016;27(12): 125704.

[19] Kim S, Ryu S. Thickness-dependent native strain in graphene membranes visualized by Raman spectroscopy. Carbon. 2016;100: 283-290.

[20] Ni ZH, Wang HM, Ma Y, Kasim J, Wu YH, Shen ZX. Tunable stress and controlled thickness modification in graphene by annealing. ACS Nano. 2008;2(5): 1033-1039.

[21] Mohiuddin TM, Lombardo A, Nair RR, Bonetti A, Savini G, Jalil R, et al. Uniaxial strain in graphene by Raman spectroscopy: G peak splitting, Grüneisen parameters, and sample orientation. Physical Review B. 2009;79(20): 205433.

[22] Ni ZH, Yu T, Lu YH, Wang YY, Feng YP, Shen ZX. Uniaxial strain on graphene: Raman spectroscopy study and band-gap opening. ACS Nano. 2008;2(11): 2301-2305. 
[23] Huang M, Yan H, Chen C, Song D, Heinz TF, Hone J. Phonon softening and crystallographic orientation of strained graphene studied by Raman spectroscopy. Proceedings of the National Academy of Sciences. 2009;106(18): 7304-7308.

[24] Berciaud S, Ryu S, Brus LE, Heinz TF. Probing the intrinsic properties of exfoliated graphene: Raman spectroscopy of free-standing monolayers. Nano Letters. 2009;9(1): 346-352.

[25] Xueshen W, Jinjin L, Qing Z, Yuan Z, Mengke Z. Thermal annealing of exfoliated graphene. Journal of Nanomaterials. 2013: 1-6.

[26] Ni ZH, Wang HM, Luo ZQ, Wang YY, Yu T, Wu YH, et al. The effect of vacuum annealing on graphene. Journal of Raman Spectroscopy. 2009;41(5): 479-483.

[27] Das A, Pisana S, Chakraborty B, Piscanec S, Saha SK, Waghmare UV, et al. Monitoring dopants by Raman scattering in an electrochemically top-gated graphene transistor. Nature Nanotechnology. 2008;3(4): 210-215.

[28] Casiraghi C, Pisana S, Novoselov KS, Geim AK, Ferrari AC. Raman fingerprint of charged impurities in graphene. Applied Physics Letters. 2007;91(23): 233108.

[29] Yan J, Zhang Y, Kim P, Pinczuk A. Electric field effect tuning of electron-phonon coupling in graphene. Physical Review Letters. 2007;98(16): 166802.

[30] Pisana S, Lazzeri M, Casiraghi C, Novoselov KS, Geim AK, Ferrari AC, et al. Breakdown of the adiabatic Born-Oppenheimer approximation in graphene. Nature Materials. 2007;6(3): 198-201.

[31] He KT, Wood JD, Doidge GP, Pop E, Lyding JW. Scanning tunneling microscopy study and nanomanipulation of graphene-coated water on mica. Nano Letters. 2012;12(6): 2665-2672.

[32] Shim J, Lui CH, Ko TY, Yu YJ, Kim P, Heinz TF, et al. Water-gated charge doping of graphene induced by mica substrates. Nano Letters. 2012;12(2): 648654 .

[33] Yoon D, Son YW, Cheong H. Negative thermal expansion coefficient of graphene measured by Raman spectroscopy. Nano Letters. 2011;11(8): 3227-3231. 
[34] Lee JE, Ahn G, Shim J, Lee YS, Ryu S. Optical separation of mechanical strain from charge doping in graphene. Nature Communications. 2012;3(1): 1-8.

[35] Ryu S, Liu L, Berciaud S, Yu YJ, Liu H, Kim P, et al. Atmospheric oxygen binding and hole doping in deformed graphene on a $\mathrm{SiO} 2$ substrate. Nano Letters. 2010;10(12): 4944-4951.

[36] Ochedowski O, Bussmann BK, Schleberger M. Graphene on mica-intercalated water trapped for life. Scientific Reports. 2014;4(1): 1-6.

[37] Xu K, Cao P, Heath JR. Graphene visualizes the first water adlayers on mica at ambient conditions. Science. 2010;329(5996): 1188-1191.

[38] Lippert G, Dabrowski J, Yamamoto Y, Herziger F, Maultzsch J, Lemme MC, et al. Molecular beam growth of micrometer-size graphene on mica. Carbon. 2013;52: 40-48.

[39] Lippert G, Dabrowski J, Lemme M, Marcus C, Seifarth O, Lupina G. Direct graphene growth on insulator. Physica status solidi (b). 2011;248(11): 26192622. 


\section{Chapter 6}

\section{Investigating the degradation of mica after high temperature annealing}

\subsection{Introduction and motivation}

In Chapter 5, AFM revealed a degradation of the mica surface after annealing graphene on mica at $500^{\circ} \mathrm{C}$. As this is an unexpected and previously unreported change of the surface during annealing, and the temperature at which this process begins is unclear, in this chapter AFM is used to investigate the bare mica surface after each annealing stage, to clarify the exact changes that occur.

Despite extensive searches, several aspects of the changes that occur in muscovite mica during heating are still unclear. Mica is reported to be resistant to thermal decomposition at temperatures around $500^{\circ} \mathrm{C}$ [1-3]. Hidnert and Dickson [4], for example, demonstrate that heating at $600^{\circ} \mathrm{C}$ for one hour produces slight or no change in the properties of muscovite samples. Another study found that heating mica at high temperatures up to $900^{\circ} \mathrm{C}$ caused interesting changes in its properties, such as changes in its optical transparency and colour of appearance [5]. Moreover, X-ray diffraction (XRD) has identified no significant structural changes up to $800^{\circ} \mathrm{C}$, whereas thermogravimetric analysis (TGA) has identified small weight loss due to dehydroxylation in mica, from around $400^{\circ} \mathrm{C}$ up to $900^{\circ} \mathrm{C}$, followed by decomposition [5]. However, the impact of annealing on the morphology of mica is still not fully understood. Consequently, this study has been unable to answer the question arising from the literature in Chapter 5, as to when, how and why mica degrades after heating at $500^{\circ} \mathrm{C}$. To address this gap, bare mica is investigated after each heating temperature at $200^{\circ} \mathrm{C}$, $300^{\circ} \mathrm{C}, 400^{\circ} \mathrm{C}$ and $500^{\circ} \mathrm{C}$, with the changes in surface structure monitored using AFM.

\subsection{Experiment}

\subsubsection{Cleaning and preparation of samples}

Firstly, bare mica was cleaved. A sharp razor blade was used to make an incision in the corner of the mica. The layered crystalline material was then split in half using tweezers. The previously internal surfaces were thus exposed for investigation (rather 
than those that were already exposed before cleaving). This method is found to be better than other methods in producing clean and flat surfaces of mica, as explained in Chapter 4.

\subsubsection{Characterisation}

AFM was employed in tapping mode to characterise the topography of the graphene sample. The measurements were performed under ambient conditions using a sharp AFM tip.

\subsubsection{Experimental procedure}

Before annealing, the cleaved mica was imaged by AFM to ensure the flatness of the sample. Then, the same sample was annealed at temperatures of $200^{\circ} \mathrm{C}, 300^{\circ} \mathrm{C}, 400^{\circ} \mathrm{C}$ and $500^{\circ} \mathrm{C}$ for one hour, in a UHV environment. After each annealing step, the sample was imaged by AFM then returned to the UHV chamber for another thermal treatment. The sample was scanned in various areas to confirm the results. The razor blade was cleaned with acetone and IPA, then dried by a nitrogen gun before being used to cleave the mica. The sample container and tweezers were cleaned in the same way to ensure that no contaminants were introduced to the exposed mica surface.

\subsection{Results}

Figure 6.1 shows the AFM images of the sample before and after annealing at different temperatures using the same colour scale. It can be observed that over the scan size, the mica is very flat and no particles are detected before annealing. Because the sample surface is atomically flat, the image shows only the background noise. No changes were detected at $200^{\circ} \mathrm{C}$ and $300^{\circ} \mathrm{C}$. However, some particles appeared after annealing at $400^{\circ} \mathrm{C}$, indicated using arrows. The particles increased dramatically on the surface of the samples after annealing at $500^{\circ} \mathrm{C}$. The size of the particles was less than $1 \mathrm{~nm}$ with a random distribution. 

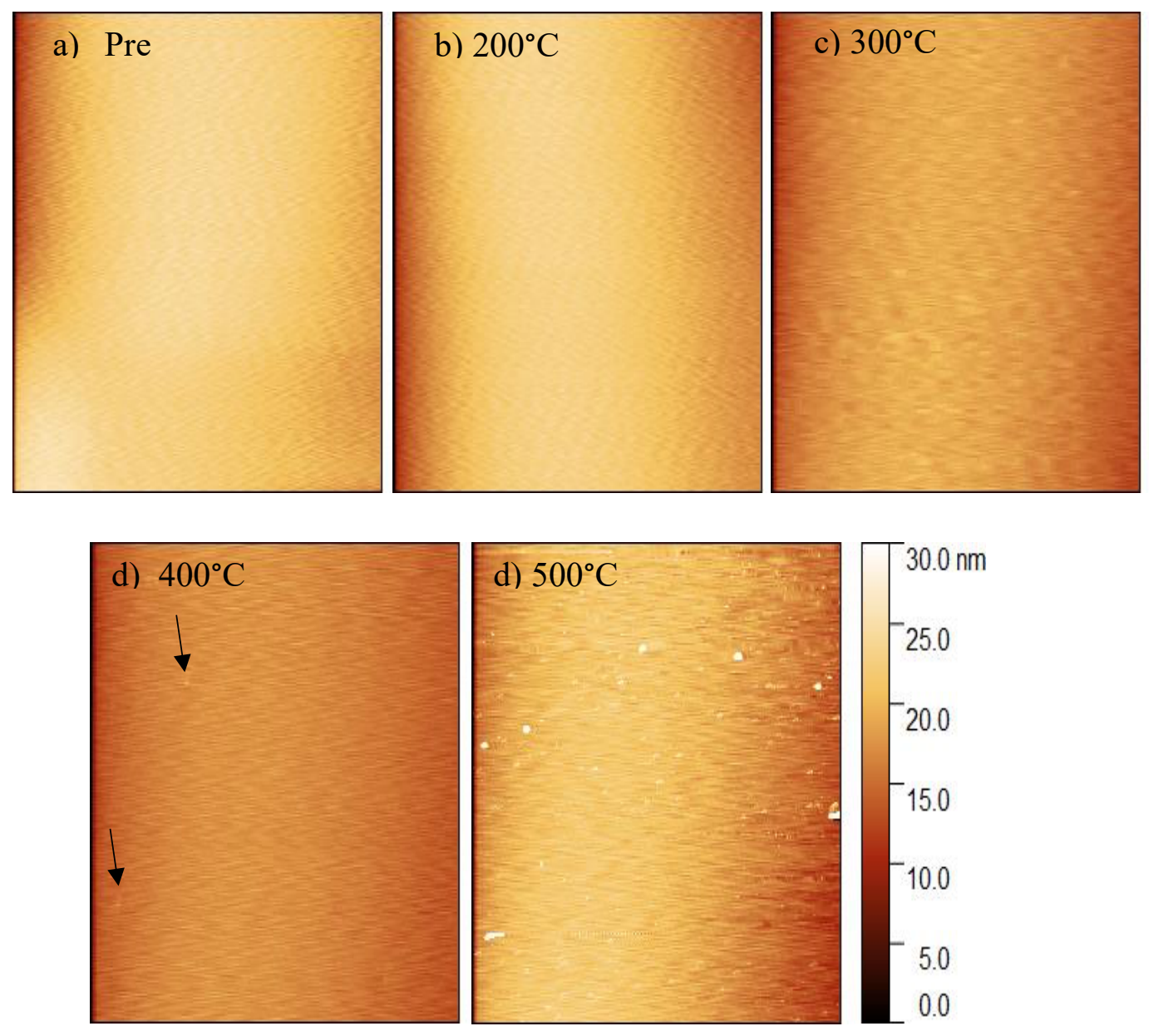

Figure 6.1: AFM images of bare mica substrate before and after the annealing process. Scan size $10 \mu \mathrm{m}$ x $10 \mu \mathrm{m}$, all on the same height scale.

\subsection{Discussion}

First: Discussion of the extremely flat surface of freshly cleaved mica before

\section{heating}

The AFM images show an extremely flat mica surface after cleavage in ambient conditions. Comparing this result with other studies, it is found that air-cleaved mica has a high density of potassium carbonate crystallites, formed immediately after cleavage by a reaction between atmospheric $\mathrm{CO}_{2}$, the mica surface and water, to produce potassium ions distributed randomly in $1-5 \mathrm{~nm}$ hexagonally shaped islands detected with AFM [6-8]. This research did not detect any particles after mica cleavage. The AFM showed a flat surface with no presence of potassium carbonate crystallites. The flatness in the cleaved mica is confirmed by de Poel et al, who used 
optical microscopy, XRD and AFM to compare two qualities of mica with respect to the number of defects, incorporated impurities and cracks, as possible sources of steps during cleaving [9]. That paper studies the difference between low- and high-quality muscovite micas in terms of the number of trapped contaminants and defects. The lowquality muscovite mica resulted in a very contaminated sample requiring the most steps, evidenced by the crack in the mica due to the pseudo-hexagonal symmetry of the crystal that commonly occurs in lower-symmetry crystal structures. The number of contaminations and defects is a good indication of how step-free the cleaved muscovite mica surface will be [9]. Furthermore, earlier studies find that the mica surface after cleavage in ambient conditions is perfectly flat with no evidence of defect following an X-ray structural analysis [10]. Although there is a step with $0.1 \mathrm{~nm}$, due to $\mathrm{K}^{+}$ions after cleavage, these are unstable and disappear after a few minutes [11]. Cleavage methods are another factor: the blade could produce imperfect cleavage, leading to inhomogeneity of the density distribution of potassium ions. This leads to hydrophilic properties that trigger the formation of $\mathrm{K}_{2} \mathrm{CO}_{3}$ islands on the mica surface when ambient water is present $[7,12,13]$.

\section{Second: Discussion of mica morphology after annealing}

After annealing at $200^{\circ} \mathrm{C}$ and $300^{\circ} \mathrm{C}$, the mica surface indicated no changes. Some clusters were observed after annealing at $400^{\circ} \mathrm{C}$, which subsequently increased at $500^{\circ} \mathrm{C}$, with particle heights of less than $1 \mathrm{~nm}$. This is an abnormal feature arising from an extremely flat surface before annealing, measured using AFM. This is the first study to show these particles appearing after annealing using AFM, with the literature indicating that the particles are produced after cleavage and before any thermal process. As such, the temperature at which these clusters appear needs to be studied. Using XRD, Hidnert et al. observe no changes in the structure of muscovite mica at annealing temperatures of $100^{\circ} \mathrm{C}$ and $250^{\circ} \mathrm{C}$ [4]. This observation agrees with the AFM images produced during this research.

One study has attempted to prevent the formation of the airborne layer of potassium carbonate particles produced after cleaving mica in air. The mica was cleaved in UHV rather than air conditions and then imaged by dynamic AFM under the same conditions. It was found that the surfaces formed by cleaving in such conditions cannot be imaged with non-contact atomic force microscopy (NC-AFM), due to large surface charges of potassium ions [13]. Nevertheless, cleavage in air produces much less 
surface charge and allows for NC-AFM imaging, because the reaction of the mica surface with ambient conditions immediately compensates for the potassium excess charge. However, mica cleaved under UHV, then exposed to air, has a similar surface morphology to air-cleaved mica samples [13].

Other studies of air-cleaved mica have attempted to remove the potassium carbonate particles by heating mica samples. It has been found that the contamination layer of potassium carbonate particles cannot be removed by degassing in UHV. Even prolonged heating under UHV conditions at a temperature of $560 \mathrm{~K}$ did not produce an atomically flat surface [13]. Moreover, a study of the surface of cleaved mica using Auger spectroscopy shows that a carbon compound was the major surface contaminant on air-cleaved mica, and that this could not be removed through heat treatment in UHV at $500^{\circ} \mathrm{C}$ [14]. In contrast, long-term heating in an atmosphere of oxygen at $10^{5} \mathrm{Torr}$ and $450^{\circ} \mathrm{C}-500^{\circ} \mathrm{C}$ removed the carbonaceous material and caused some loss of surface potassium [14]. This paper observed that the carbonaceous material was already formed, regardless of whether the mica was cleaved in the air at the beginning, or it was cleaved in an ultra-high vacuum (UHV) and subsequently exposed to air. In addition, carbonaceous surface layers are not produced in the case of UHV-cleaved mica exposure to carbon dioxide $\left(\mathrm{CO}_{2}\right)$, methane $\left(\mathrm{CH}_{4}\right)$ or carbon monoxide $(\mathrm{CO})$, as this layer forms only when water vapour is present $[14,15]$. Another study heated mica at $600-900 \mathrm{~K}$ but could not remove the particles, as confirmed by static secondary-ion mass spectrometry (SSIMS) $[13,16]$. Other SSIMS studies comparing freshly cleaved and weathered mica surfaces have demonstrated a depletion of potassium from mica surfaces through weathering under ambient conditions, because of an increase in surface oxygen $[15,17]$. This is consistent with the formation and clustering of potassium carbonate on the surface. In addition, it is evidenced that carbon dioxide may adsorb on the surface of mica cleaved in a vacuum, whereas air-cleaved mica does not attract carbon dioxide [15,18]. This is because mica cleaved in ambient conditions already has a carbonaceous layer on its surface, as confirmed by X-ray photo electron spectroscopy (XPS) investigation $[15,19]$. However, annealing the mica surface causes loss of weight due to of dehydroxylation, as shown in Equation 6.1. Continued annealing of the mica will lead to decomposition [5]. 


\section{$\mathrm{KAl}_{2}\left(\mathrm{AlSi}_{3}\right) \mathrm{O}_{10}(\mathrm{OH})_{2} \stackrel{\Delta}{\rightarrow} \mathrm{KAl}_{2}\left(\mathrm{AlSi}_{3}\right) \mathrm{O}_{11}+\mathrm{H}_{2} \mathrm{O} \uparrow$}

Equation 6.1: dehydroxylation in mica [5]

Overall, annealing mica in a UHV environment causes changes in the mica surface. This is evidenced by the presence of a few particles with a height of less than $1 \mathrm{~nm}$ after annealing at $400^{\circ} \mathrm{C}$, which increase in number with annealing at $500^{\circ} \mathrm{C}$, in a random distribution. These particles are formed from the mica surface itself, because annealing mica causes loss in its surface layer. This study relies on strong evidence indicating that annealing in oxygen at $450^{\circ} \mathrm{C}-500^{\circ} \mathrm{C}$ results in a loss of carbonaceous material and surface potassium, according to Auger spectroscopy [14,15]. Auger spectroscopy probes the electronic energy levels of ions undergoing autoionization uniquely identifying each element and is fundamentally a surface probe as the data comes from approximately the top five atom layers [20]. Other evidence using TGA shows a small amount of weight loss when annealing mica at around $400^{\circ} \mathrm{C}$, due to dehydroxylation [5]. Therefore, the best annealing temperature for mica substrate is $300^{\circ} \mathrm{C}$, where no surface changes have been detected.

\subsection{Conclusions}

In Chapter 5 unexpected and previously unreported changes occurred on the surface of mica after annealing, where small particles were observed using AFM after the final annealing step of $500{ }^{\circ} \mathrm{C}$. In this chapter, all intermediate temperature stages of before annealing, $200{ }^{\circ} \mathrm{C}, 300{ }^{\circ} \mathrm{C}, 400{ }^{\circ} \mathrm{C}$ and $500{ }^{\circ} \mathrm{C}$ are studied on bare mica to avoid any effects of graphene deposition. Atomically flat terraces of $10 \mu \mathrm{m}$ x $10 \mu \mathrm{m}$ are scanned with AFM and compared.

The as-cleaved and un-annealed surface showed no surface changes, with apparently atomically flat, uncontaminated surfaces present. At $400{ }^{\circ} \mathrm{C}$ particulates around $1 \mathrm{~nm}$ in size appeared on the surface, which increased at $500{ }^{\circ} \mathrm{C}$. This is the first study to show particle appearance with annealing.

Other studies have found a contamination layer of potassium carbonate crystallites on mica immediately after cleaving. Annealing those samples showed a reduction in the number of crystallites which were around $1-5 \mathrm{~nm}$, with a corresponding reduction in the mass of mica. Other studies using thermogravimetric analysis showed a small 
amount of weight loss when annealing mica at around $400{ }^{\circ} \mathrm{C}$, attributed to dehydroxylation.

These results, coupled with the previous chapter, show both related effects must be occurring simultaneously. We form clean-cleaved surfaces of mica with no surface crystallites, but these are induced by annealing to $400{ }^{\circ} \mathrm{C}$. Further, the AFM height analysis of graphene on mica in the previous chapter showed that the background height of the mica around the graphene reduced after annealing at $500{ }^{\circ} \mathrm{C}$, and therefore loss of material is also occurring. This is the first observation of these effects.

For the purposes of this work, it now establishes an upper limit on the annealing temperature used to clean graphene on mica of $300{ }^{\circ} \mathrm{C}$. By coincidence, this matches the upper limit for annealing graphene on $\mathrm{SiO}_{2}$, although the mechanisms which establish these two limits are completely different for each material system. 


\subsection{References}

[1] Gaines GL, Vedder W. Dehydroxylation of muscovite. Nature. 1964;201(4918): 495.

[2] Heller-Kallai L, Lapides I. Dehydroxylation of muscovite: Study of quenched samples. Physics and Chemistry of Minerals. 2015;42(10): 835-845.

[3] Ochedowski O, Bussmann BK, Schleberger M. Graphene on mica-intercalated water trapped for life. Scientific Reports. 2014;4(1): 1-6.

[4] Hidnert P, Dickson G. Some physical properties of mica. Journal of Research of the National Bureau of Standards. 1945;35(4): 309.

[5] Kirubanithy M, Sivanantham N, Gopalakrishnan N, Balamurugan K. Effect of heat treatment on the optical properties of layered muscovite single-crystal sheets. Bulletin of Materials Science. 2014;43(1): 1-8.

[6] Christenson HK, Israelachvili JN. Growth of ionic crystallites on exposed surfaces. Journal of Colloid and Interface Science. 1987;117(2): 576-577.

[7] Ostendorf F, Schmitz C, Hirth S, Kühnle A, Kolodziej JJ, Reichling M. Evidence for potassium carbonate crystallites on air-cleaved mica surfaces. Langmuir. 2009;25(18): 10764-10767.

[8] Israelachvili JN, Alcantar NA, Maeda N, Mates TE, Ruths M. Preparing contamination-free mica substrates for surface characterization, force measurements, and imaging. Langmuir. 2004;20(9): 3616-3622.

[9] de Poel W, Pintea S, Drnec J, Carla F, Felici R, Mulder P, et al. Muscovite mica: Flatter than a pancake. Surface Science. 2014;619: 19-24. Available from: dx.doi.org/10.1016/j.susc.2013.10.008.

[10] Baba M, Kakitani S, Ishii H, Okuno T. Fine atomic image of mica cleavage planes obtained with an atomic force microscope (AFM) and a novel procedure for image processing. Chemical Physics. 1997;221(1-2): 23-31.

[11] Campbell PA, Sinnamon LJ, Thompson CE, Walmsley DG. Atomic force microscopy evidence for $\mathrm{K}+$ domains on freshly cleaved mica. Surface Science. 1998;410(2-3): L768-L772. 
[12] Hattendorf S, Georgi A, Liebmann M, Morgenstern M. Networks of ABA and ABC stacked graphene on mica observed by scanning tunnelling microscopy. Surface Science. 2013;610: 53-58.

[13] Ostendorf F, Schmitz C, Hirth S, Kühnle A, Kolodziej JJ, Reichling M. How flat is an air-cleaved mica surface? Nanotechnology. 2008;19(30): 305705.

[14] Poppa H, Elliot AG. The surface composition of mica substrates. Surface Science. 1971;24(1): 149-163.

[15] Christenson HK, Thomson NH. The nature of the air-cleaved mica surface. Surface Science Reports. 2016;71(2): 367-390.

[16] Dowsett MG, King RM, Parker EHC. Evaluation of impurity and contamination levels on mica surfaces using SSIMS. Journal of Vacuum Science and Technology. 1977;14(2): 711-717.

[17] Baun WL. ISS/SIMS characterization of mica surfaces. Surface and Interface Analysis. 1980;2(4): 145-147.

[18] Bhattacharyya KG. Adsorption of carbon dioxide on mica surfaces. Langmuir. 1989;5(5): 1155-1162.

[19] Bhattacharyya KG. XPS study of mica surfaces. Journal of Electron Spectroscopy and Related Phenomena. 1993;63(3): 289-306.

[20] Chang CC. Auger electron spectroscopy. Surface Science. 1971 Mar 1;25(1):5379. 


\section{Chapter 7}

\section{E-beam lithography of mica and suspended graphene}

\subsection{Introduction}

Two-dimensional (2D) materials - i.e. atomically thin crystalline materials, such as graphene - offer strong potential for future flexible electronics applications, including flexible displays such as touchscreens, smart textiles and wearable biosensors. Due to the remarkable mechanical properties and electron mobility of graphene, it is currently used as a transparent and flexible conducting electrode [1-3]. The promising flexible electronics industry will need atomically thin and flexible semiconductors and insulators as well as conductors. This has motivated researchers to explore the fabrication and characterisation of atomically thin semiconductors, such as molybdenum disulphide $\left(\mathrm{MoS}_{2}\right)$ [4-8], and insulators, such as boron nitride [9-12] or muscovite mica [13-17]. Among all of these, muscovite mica, as a lightweight, lowcost and flexible transparent material, has been most explored and is used in some areas of electronics due to its high chemical/thermal stability and dielectric behaviour.

The most challenging issue in the practical application of ultrathin films is the transfer of these films from the growth substrate to the target substrate. Another important challenge is preventing the substrate destruction and sample degradation that occur as a result of the present transfer method of applying the wet chemical etching process. Mica overcomes this issue and is, therefore, an ideal candidate for the transfer of thin films because a sacrificial layer can be removed from the surface of the mica, removing any in-process contamination, and then the underlying mica can also be lifted off. This guarantees a simple transfer process due to mica's unique cleavage property that can be easily achieved through multiple re-applications of standard adhesive tape [18]. This has led to this question: what if voids could be etched into the mica to create suspended thin films after transfer with the substrate features of flexibility, dry transfer, controllable thickness and transparency?

When mica is used as an electrically insulating support for the study of nanomaterials like graphene, locating small samples repeatedly across several instruments is made easier when location grids or markers are etched into the substrate. Typically, these need to be deep enough to provide contrast in optical and electron microscopy but not 
as deep as these proposed trenches to create the voids used for suspending nanomaterials. However, both require methods of etching the mica.

A few studies have demonstrated controlled patterning of mica substrates through mechanical or chemical methods. For instance, nanostructured patterns of different sizes and shapes have been produced on the surface of mica using the tip of an atomic force microscope (AFM), which was operated in contact mode by controlling the interaction between the tip and the scanning area. By repeatedly probing the tip along an established pattern on the surface of the mica, mechanically induced local etching could be detected at the atomic scale $[16,19]$.

However, this technique was attempted in this work but was found to have a limited etching depth and to produce extra flakes when the mica was drilled, which created another issue of the disposal of these unwanted flakes on top of what was already a time-consuming process. Figure 7.1 shows square holes of $2 \mu \mathrm{m} \times 2 \mu \mathrm{m}$ produced by mechanical etching on mica surfaces using the tip in contact mode by applying a force of $1.14 \mu \mathrm{m}$ and repeating the scanning to increase the hole depth: a) the first scan produced a hole with a depth of $4 \mathrm{~nm}$; b) repeating the scan three times produced a depth of $7 \mathrm{~nm}$; c) a non-uniformly shaped hole was created via scanning five times with a depth around $8 \mathrm{~nm}$.
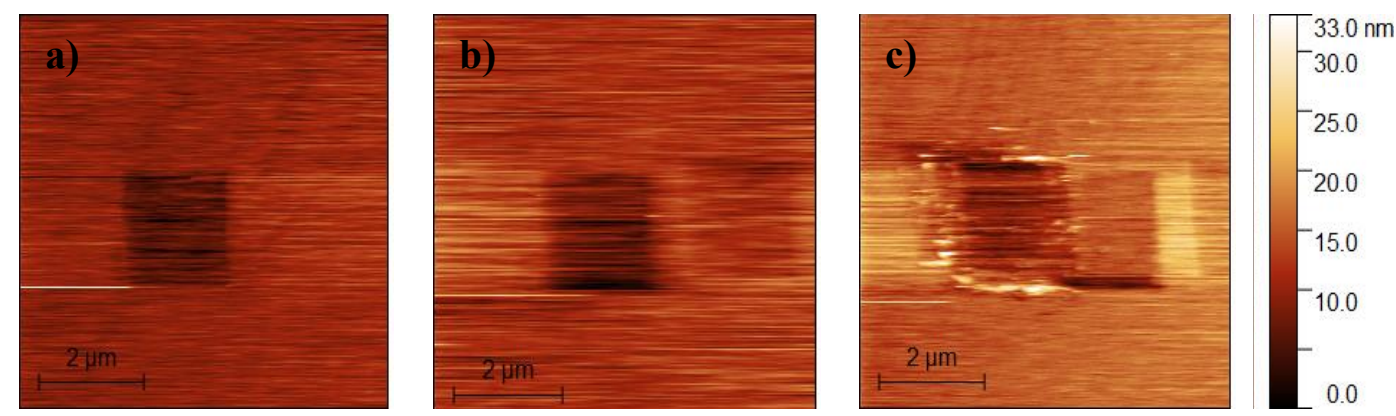

Figure 7.1: Mechanical etching of mica substrate using AFM tip.

Controlling the hole depth by tuning the scanning times: a) one, b) three, and c) five scan times.

Conventionally, mica patterning by wet etching using hydrofluoric acid (HF), as mica is a hard material to etch, may introduce some contamination to the $2 \mathrm{D}$ materials. Thus, to avoid acid etching in the fabrication process, a resist can be used as the sacrificial layer instead with dry etching, which may solve this drawback. Plasma has been used 
to etch mica without a mask or by using a physical mask, such as transmission electron microscopy (TEM) grids of different sizes. [20-25]. However, this method obstructs the nanostructure design and requires a hazardous solvent.

The limitation of designing a photolithography mask to pattern a substrate can be overcome by using electron-beam lithography (EBL). Typical EBL systems use an electron beam to sequentially write each feature in the pattern, whereas in photolithography, the entire pattern is illuminated at the same time for a few minutes. The advantages of EBL include small feature sizes of $10 \mathrm{~nm}$ or less, which typically take a longer of period time, i.e. almost hours, to pattern [26]. In addition, the mask is designed by easily adjustable software, whereas photolithography requires a physical mask designed by specialist companies. However, mica has not yet been patterned using the electron beam lithography technique. Photolithography has been used for selective-area fluorination and modification of surface free energy on mica [27] but not for the required etching.

Here, the substrate fabrication process was refined and iterated many times over the course of the $\mathrm{PhD}$, and a method for etching mica using lithography was established for the first time. There were too many iterations for them all to be shown in this thesis. However, the principal challenges that were faced and their resolutions are discussed below along with the experiments performed to understand each issue. These are all brought together in the final section, which shows the successful final method for the patterning lithography (PL) etching of mica, the first report of such a process. After that, the successfully patterned mica characteristics using optical microscopy and AFM techniques are demonstrated. Finally, the suspended graphene sheets on the patterned mica substrate are presented. We believe this work represents the first lithography of a mica substrate as well as the first suspended graphene on patterned mica.

\subsection{Experimental methods}

As described in Chapter 2, the process of lithography starts by coating a substrate surface with an exposure-sensitive material. Then, a patterned mask is applied to the substrate to block exposure of some areas; thus, unmasked regions of the resist will be exposed. A developer, i.e. a solvent, is applied to the surface to remove unhardened areas. In the case of a negative resist, the exposed regions, i.e. the unmasked regions, 
will harden, and the developer will only dissolve the areas that were not exposed, leaving behind a coating in the regions where the mask was not placed. In contrast, with a positive resist, the exposed areas will be degraded, and the developer will dissolve away the areas that were exposed, leaving behind a coating where the mask was placed.

Figure 7.2 below shows the main stages, including the lithography and etching. To start, the desired mask was drawn using the Raith software. Then, the sample preparation started: fresh, horizontally cleaved mica substrate was immediately coated with a negative (positive) electron resist using a spin coater. After that, pre-exposure baking (a soft bake) occurred to improve the adhesion between the resist and the substrate [28]. Subsequently, the sample was loaded into a Raith e-LiNE chamber. After all the required alignment and exposure settings were completed, the sample was left for the required time until the exposure was complete. Once the sample was removed from the chamber, the post-exposure bake (PEB) (a hard bake) was required in order to increase the thermal, chemical and physical stability of the developed resist structures $[29,30]$; however, PEB was not required in the case of a positive resist. Next, the sample was left for a few minutes to hydrate - a delay for water resorption. Development was then performed to dissolve the unhardened masked regions and leave the hardened unmasked regions behind. Then, the etching process began, with a reactive ion etcher (RIE Oxford Plasma Lab 80 Plus) used for plasma etching, a form of dry etching, to etch the unprotected resist regions. Finally, all remaining layers were removed using stripper solvents. However, corresponding to the issues that were faced, these stages were adjusted, with changes in the types of chemicals used, the arrangement of the coated layers and the process order. 


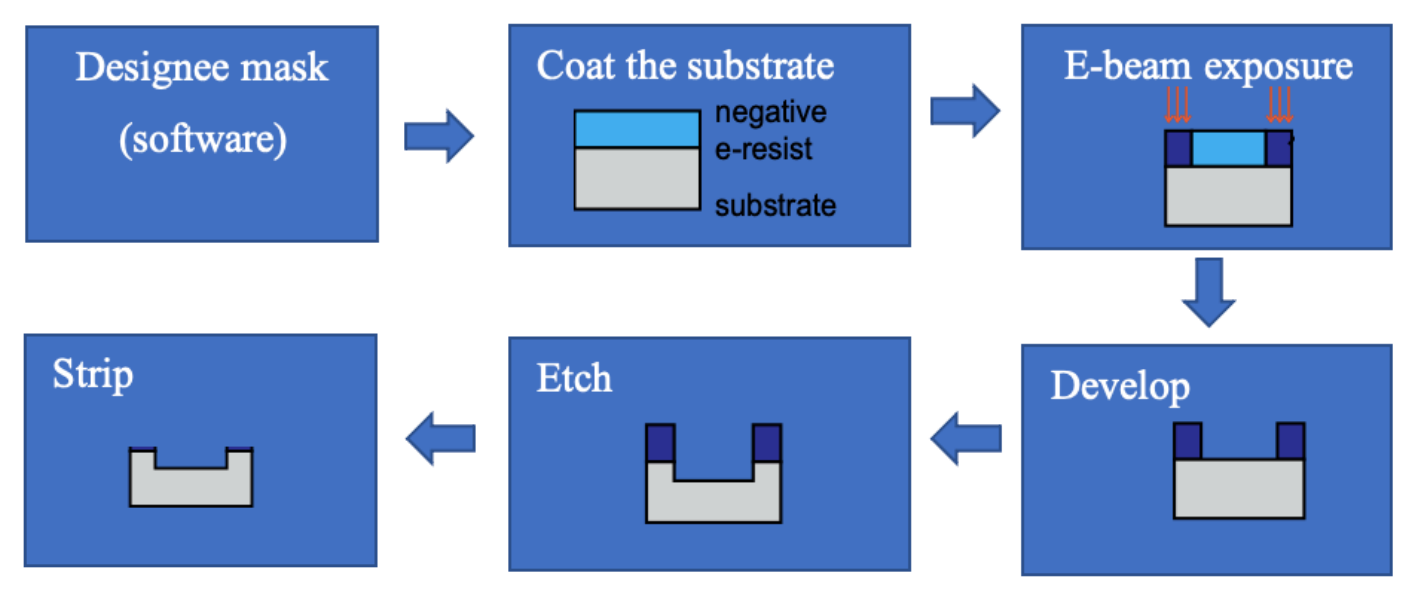

Figure 7.2: Patterning mica procedures

\subsection{Results and discussion}

\subsubsection{The problem of the adhesion between the mica and the resist layer or any other layer}

The first significant issue faced in patterning the mica was the poor adhesion between the mica surface and any overlayer. This was observed when a sample of a fresh, horizontally cleaved mica was coated with AZ nLOF 2070 (purchased from MicroChemicals), a negative resist. After performing electron-beam (e-beam) exposure and the development process in the diluted developer of AZ $726 \mathrm{MIF}$ (purchased from MicroChemicals), the optical microscopy did not detect any patterns on the mica surface, as the developer had completely removed the entire pattern. Therefore, the resist adhesion and substrate pre-treatment were investigated in the next step to eliminate this major issue.

However, when the same recipe and the same EBL setting were tested on a silicon substrate (washed with acetone, isopropyl alcohol (IPA) and deionised (DI) water and dried with a nitrogen gun), the optical image showed that the pattern was deposited and developed successfully, with four different pattern areas with varying exposure doses $\left(20,30,40,50 \mu \mathrm{C} / \mathrm{cm}^{2}\right)$ (see Figure 7.3). 


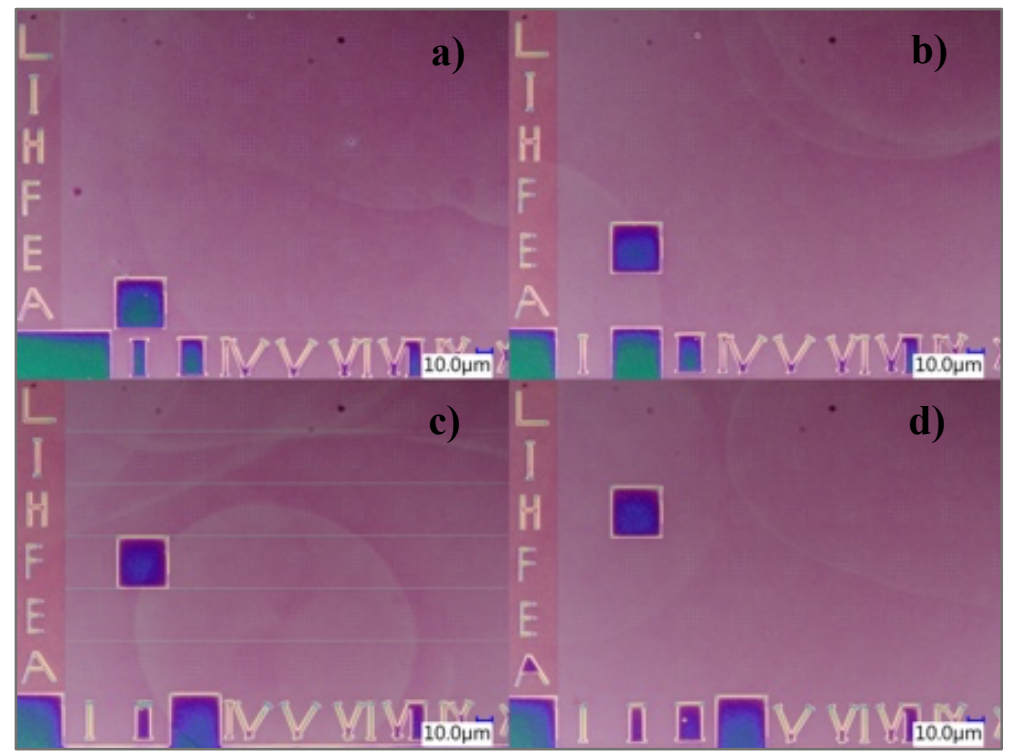

Figure 7.3: Optical microscopy image of silicon/nLOF 2070 resist by e-beam lithography with four different exposure doses a) 20 , b) 30 , c) 40 and d) $50 \mu \mathrm{C} / \mathrm{cm}^{2}$

In order to solve this issue and improve the adhesion between the mica and the resist, an extra adhesion layer was deposited on the mica substrate prior to the application of the resist. For example, chromium $(\mathrm{Cr})$ sputter deposited on the mica substrate was considered an adhesion layer. Here, a 10-nm-thick coating of Cr sputter (Quorum) was deposited between the mica surface and the resist layer, which improved the adhesion between the layers. Then, exposure was performed, and the sample was developed in diluted AZ 726. After a few seconds in the developer, the pattern visibly started peeling off from the mica, as shown in the optical image below (Figure 7.4), and within a minute, the pattern had disappeared. This suggested either poor adhesion between the $\mathrm{Cr}$ and mica, or that the mica was being etched in the Tetra Methyl Ammonium Hydroxide (TMAH)-based developer or both. 


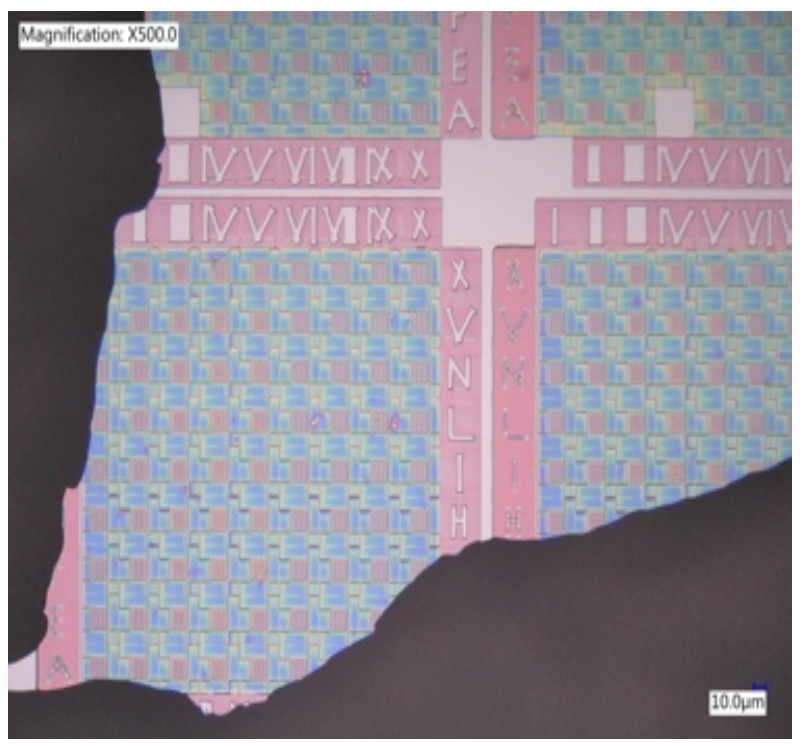

Figure 7.4: Optical microscopy image of mica/Cr/nLOF 2070 resist. The pattern started peeling off after a few seconds in the developer solvent.

For this reason, a set of experiments was designed to test the adhesion of the $\mathrm{Cr}$ and mica using a different treatment on the mica as well as to investigate the different types of developers and different preparation methods. The next section will introduce a positive resist - poly (methyl methacrylate) (PMMA) - to the sample preparation.

In this manner, a freshly cleaved mica sheet was coated with $100 \mathrm{~nm}$ of $\mathrm{Cr}$, a thicker layer than was used in the previous test, with no resist on top. As shown in Table 7.1, tests 1, 2 and 3 used the same cleaved substrate without any treatment such as cleaning or annealing; however, different types of developers were used. The results indicated that none of the developers were promising on the untreated mica.

In order to remove any contamination that could affect the adhesion, in test 4 , the sample was treated with a cleaning procedure: to start, the cleaved mica was dipped in acetone and then washed with IPA prior to the $\mathrm{Cr}$ deposition. This sample was tested with the diluted developer methyl isobutyl ketone (MIBK), which is generally the recommended developer for PMMA (tested in the experiments explained in the next section). A comparison of the results from tests 3 and 4 using the same developer of 
MIBK demonstrated that the cleaning procedure by solvents had no beneficial effect on the $\mathrm{Cr}$ /mica adhesion. In contrast, when comparing the results of tests 5 and 6 , when the mica substrate was treated with annealing at $200^{\circ} \mathrm{C}$ for two minutes before $\mathrm{Cr}$ deposition and two different types of developers were used, the results were promising.

Table 7.1: Test the adhesion of mica /Cr $100 \mathrm{~nm}$ using different treatment

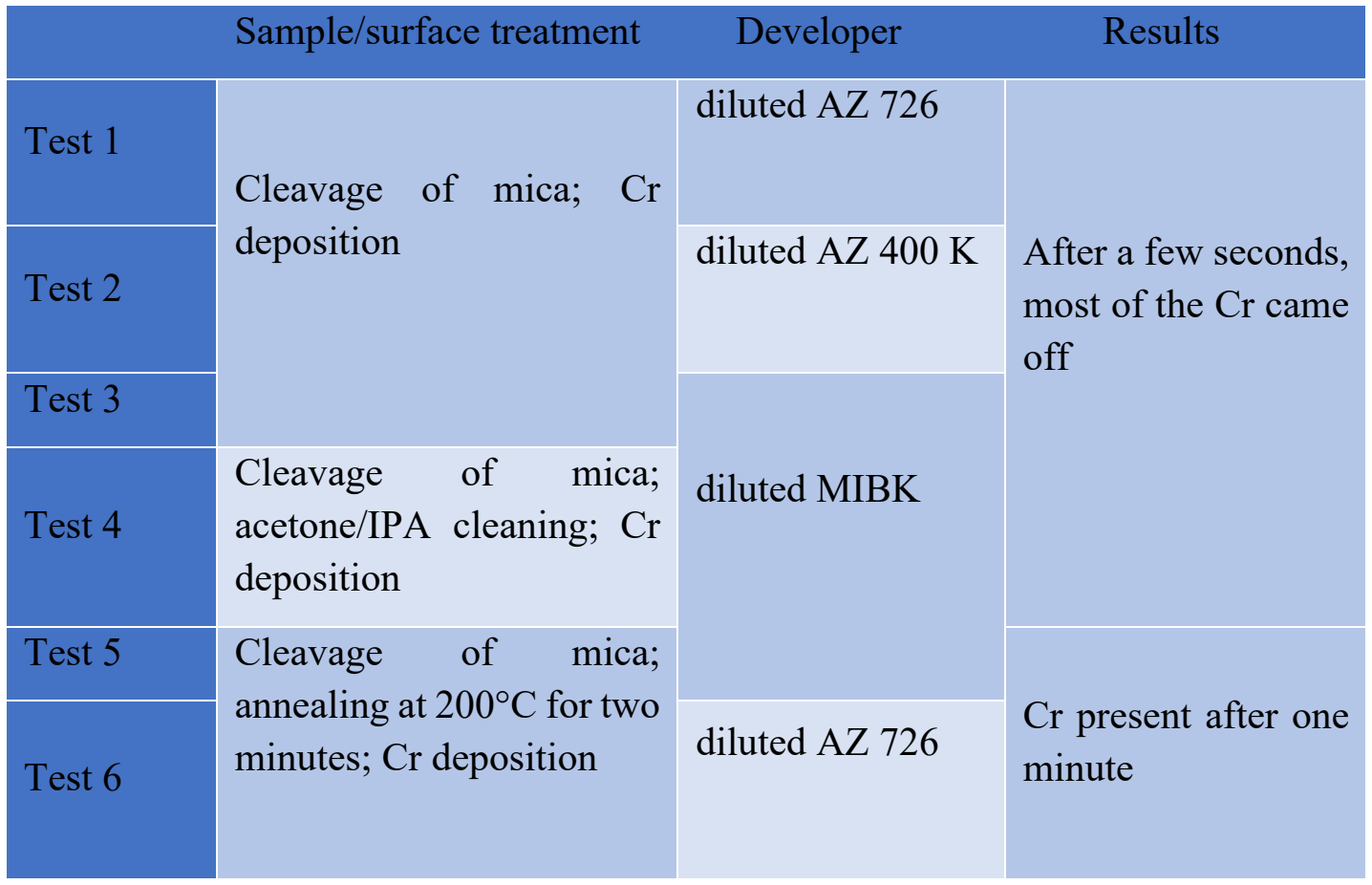

The results reported here indicate that the annealing improved the adhesion between the $\mathrm{Cr}$ and the cleaved mica substrate. In case the substrates had become contaminated with organic impurities, a cleaning process was performed using acetone and IPA solvents. This step was carried out in test 4 but did not successfully improve the adhesion, which indicated that no organic impurities were present. Therefore, the best strategy to eliminate the adhesion issue was to anneal the cleaved mica at $200^{\circ} \mathrm{C}$ for two minutes before depositing the layer of $\mathrm{Cr}$. From here, the substrate surface depended on the amount of relative humidity in the environment since a water film can be adsorbed immediately. In this work, horizontally cleaving the mica was not enough to remove any contamination layer and an annealing step was needed, while in another study [27], cleaved mica was sufficient. However, after the substrate was annealed, it needed to be cooled before any further deposition steps could take place. 


\subsubsection{The problem of pattern invisibility under optical microscopy}

The next issue was the inability to detect the pattern on the mica substrate using optical microscopy. This issue arose when the recipe of the sample preparation was changed. It was important to optimise the recipe to test whether or not the mica surface successfully adhered to any layer. Here, $\mathrm{Cr}$ was used as an adhesion promoter and modified the substrate surface with regard to the optimised resist wetting and adhesion. While the Cr layer successfully adhered to the annealed mica surface, it was important to check if this included the adhesion of the resist to the mica substrate - the resist would be on top of the mica. Thus, for comparison purposes, a different order of coated layers was used to confirm the successful adhesion of any layer onto the mica surface. To start, the mask was drawn with the Raith software; then, the mica substrate was cleaved, followed by the annealing process. Next, the mica substrate was coated with PMMA resist (MicroChemicals) using a spin coater, followed by the soft bake. After that, a $\mathrm{Cr}$ layer was deposited on sample 1 (S1) (mica/PMMA/5 nm Cr). The same parameters of the cleaved/annealed mica, $\mathrm{Cr}$ thickness and PMMA layer were applied to a second sample (S2) (mica/5 nm Cr/PMMA); however, the $\mathrm{Cr}$ layer was the first layer, followed by an e-resist layer. The PMMA resist was chosen because it can process any layer in white light; however, negative resist nLOF 2070 is sensitive to white light and, therefore, had to be processed and developed in yellow light. After the sample preparation, e-beam exposure was carried out. The exposure to the e-beam divided the polymer into fragments that could be dissolved in developer MIBK. However, MIBK is a very strong developer when used alone and may remove some of the unexposed resist. Thus, a mixture of one part MIBK to three parts isopropanol was used. The sample was then rinsed with pure IPA and dried with a nitrogen gun.

Here, S2 only required developing to pattern the resist layer, while $\mathrm{S} 1$ required the $\mathrm{Cr}$ layers to be removed first using $\mathrm{Cr}$ etchant (purchased from MicroChemicals), followed by developing to pattern the resist. However, the pattern in S2 could be seen by optical microscopy (HVX- x50), while that in S1 could not be seen, leading to another problem. In Figure 7.5 (left), optical microscopy did not show the pattern in $\mathrm{S} 1$, but after optimising the contrast (right), the pattern was shown, with the Cr layer reflecting the light. The lithography processes for both samples are shown in Figure 7.6. Both samples were successfully patterned using different preparation recipes. This confirmed that the cleaved/annealed mica surface adhered well to any layer on top of 
it, with or with without using an adhesion promoter between the mica and the other layer.
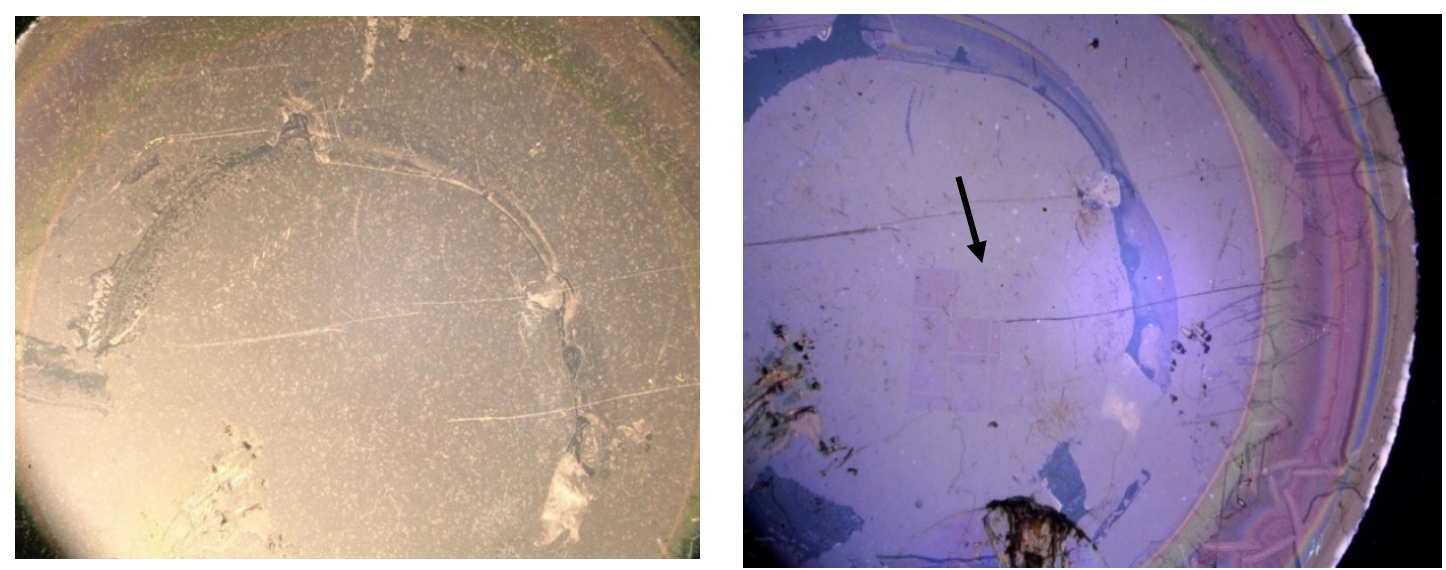

Figure 7.5: Optical microscopy image of S1 (mica/PMMA/Cr). Left: the pattern is invisible under the normal settings of optical microscopy. Right: The pattern shown after optimising the contrast 


\section{Drawing mask by Raith software}

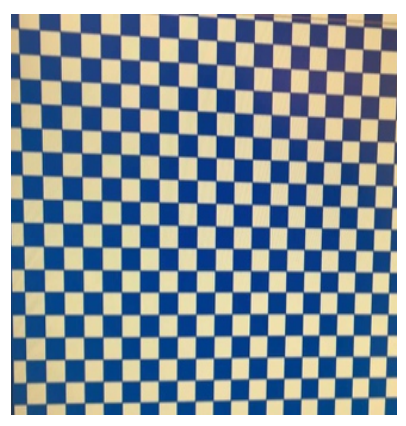

\section{Coating samples by two layers}
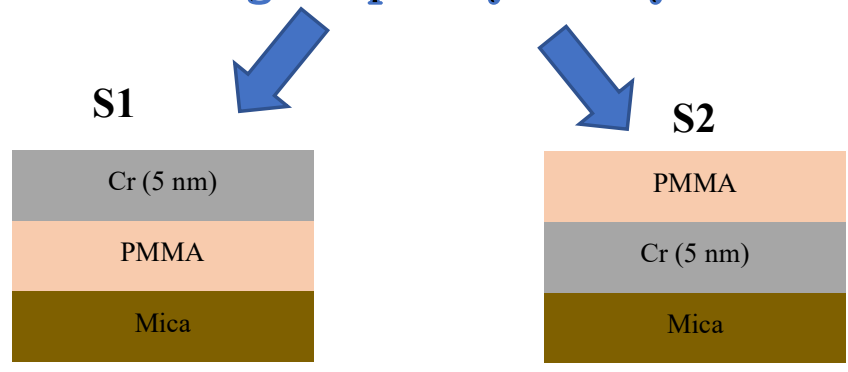

\section{e-beam exposure}

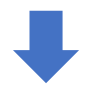

Cr etchant

Developing
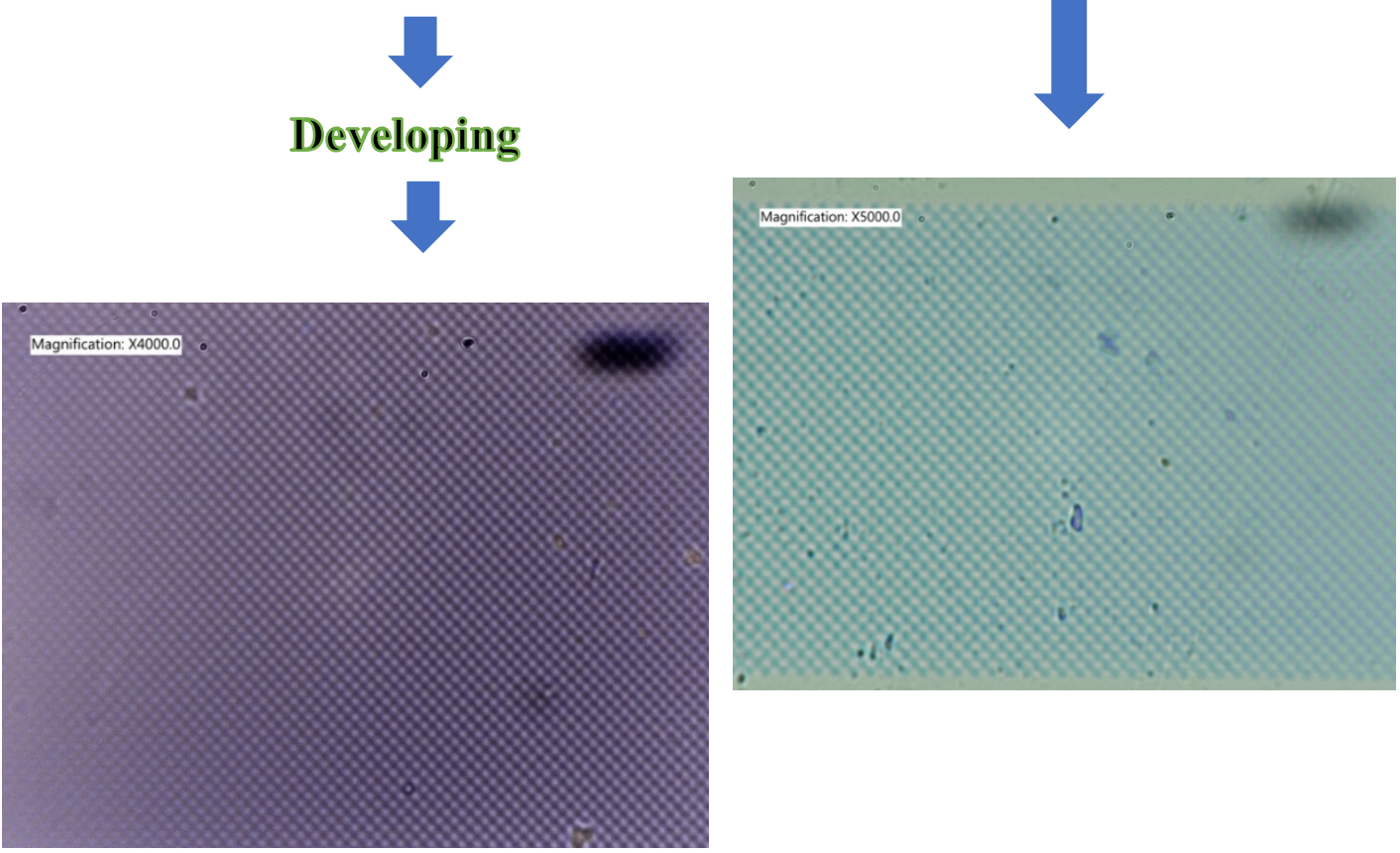

Figure 7.6: Optical microscopy image of the lithography of the patterns in the mica in different prepration processes coated the sample with two layers. Left: S1 (mica/PMMA/Cr). Right: S2 (mica/Cr/PMMA) 


\subsubsection{Testing recipes}

After the adhesion issue between the mica and coated layers was tackled and the settings for the optical microscopy for detecting the patterned mica were optimised, the lithography recipes to pattern the mica substrate were established.

\section{A: Lithography process steps for the first recipe}

This section describes the establishment of the preparation recipe, exposure dose, baking and developing time and plasma etching parameters.

Initially, the mica disk substrates were cleaved horizontally and then annealed at $200^{\circ} \mathrm{C}$ for two minutes to remove contamination. It should be noted that it was necessary to be very careful with the mica substrates, as they were transparent, and to keep track of the front side, i.e. the freshly cleaved side, of the samples. Next, to prevent the mica surface from reacting with any chemical processes such as a developer, which could slow the etching of the surface, a layer of PMMA A4 495 was spin-coated at 4,000 rpm for one minute on top of the substrate. The substrate was then baked on a hotplate at $180^{\circ} \mathrm{C}$ for two minutes, and the resist was $200-\mathrm{nm}$ thick. Then, the negative resist, AZ nLOF 2070 for e-beam, was spun at 4,000 rpm for 40 seconds on the substrate, followed by the soft pre-exposure bake at $100^{\circ} \mathrm{C}$ for one minute; the resist thickness was $500 \mathrm{~nm}$. These two layers of resist dramatically improved the lithography yield. Subsequently, the sample was loaded into a Raith e-LiNE chamber. After completing all the required alignments (all alignments were done on very small features on the corner of the sample), e-beam exposure was performed at $5 \mathrm{kV}$ acceleration with a 60 $\mu \mathrm{m}$ aperture. The exposure dose will be explained later in the thesis. After the PEB, i.e. the hard bake, at $110^{\circ} \mathrm{C}$ for one minute, the sample was left for five minutes to hydrate, a delay for $\mathrm{H}_{2} \mathrm{O}$ resorption. However, both the pre- and post-exposure baking times required further optimisation, as an issue arose during these processes.

Next, development was performed with a concentration of a diluted developer at 2:1 AZ 726 MIF: DI water, followed by 1:1 and 1:2 concentrations. Finally, the sample was quickly rinsed in pure DI water and then dried with a nitrogen gun. The best developing time was tested later, corresponding to the optimisation of the baking times.

For the purpose of transferring the patterns onto the PMMA film, the plasma etching was performed in two steps. Before the sample was loaded into the plasma chamber, 
the chamber's cleaning process was carried out using oxygen plasma $(100 \mathrm{sccm} \mathrm{O}$, $100 \mathrm{~W}, 100 \mathrm{mTorr}$ for $15 \mathrm{~min}$ ). After the sample was loaded into the clean chamber, the first process of etching was performed using oxygen to etch the PMMA layer. This was followed by the second step of etching the mica in tetrafluoromethane $\left(\mathrm{CF}_{4}\right)$ and an argon (Ar) atmosphere; the Ar was used to reduce the damage done to the surface. The etching recipes were later investigated further by changing the etch time/power/pressure.

\section{A1: The problem of deformation of the pattern at different exposure doses}

After the sample was prepared and loaded into the e-beam chamber, another issue arose: the pattern appeared to not be drawn properly. This issue worsened as the exposure dose increased. Therefore, the exposure dose had to be considered a starting point for the individual process optimisation. For a given dose, the minimum exposure time for a given area was calculated by the following formula:

$$
D . A=T . I,
$$

where $\mathrm{T}$ is the time of exposure to an object, which can be divided into exposure time/step size, I is the current pulse, D is the dose and A is the exposure area.

If the exposure dose was too high, due to scattering, the resist became more and more insoluble in the developer. If the dose was too low, the remaining resist thickness decreased more and more at the areas where the resist patterns needed to remain. The dose had to be carefully optimised for every type of substrate because the scattering of electrons can be different on different substrates. In this section, we examined two groups of exposure doses, as this was a significant parameter. Therefore, in testing, the range of the dose went from 20 to $100 \mu \mathrm{C} / \mathrm{cm}^{2}$ and was divided into two groups. To start, an examination of the group with exposure doses of $100 \mu \mathrm{C} / \mathrm{cm}^{2}$ showed that the dose was too high, and the pattern was not drawn (see Figure 7.7). In the second group, the maximum dose was $50 \mu \mathrm{C} / \mathrm{cm}^{2}$, with doses of 20 and $50 \mu \mathrm{C} / \mathrm{cm}^{2}$. With the dose of $20 \mu \mathrm{C} / \mathrm{cm}^{2}$, the pattern was almost written, but the dose of $50 \mu \mathrm{C} / \mathrm{cm}^{2}$ did not result in more details being drawn. Therefore, for both samples, this recipe did not successfully draw on the mica substrate due to the charging effect. Figure 7.8 shows the comparison of these two exposure doses. 

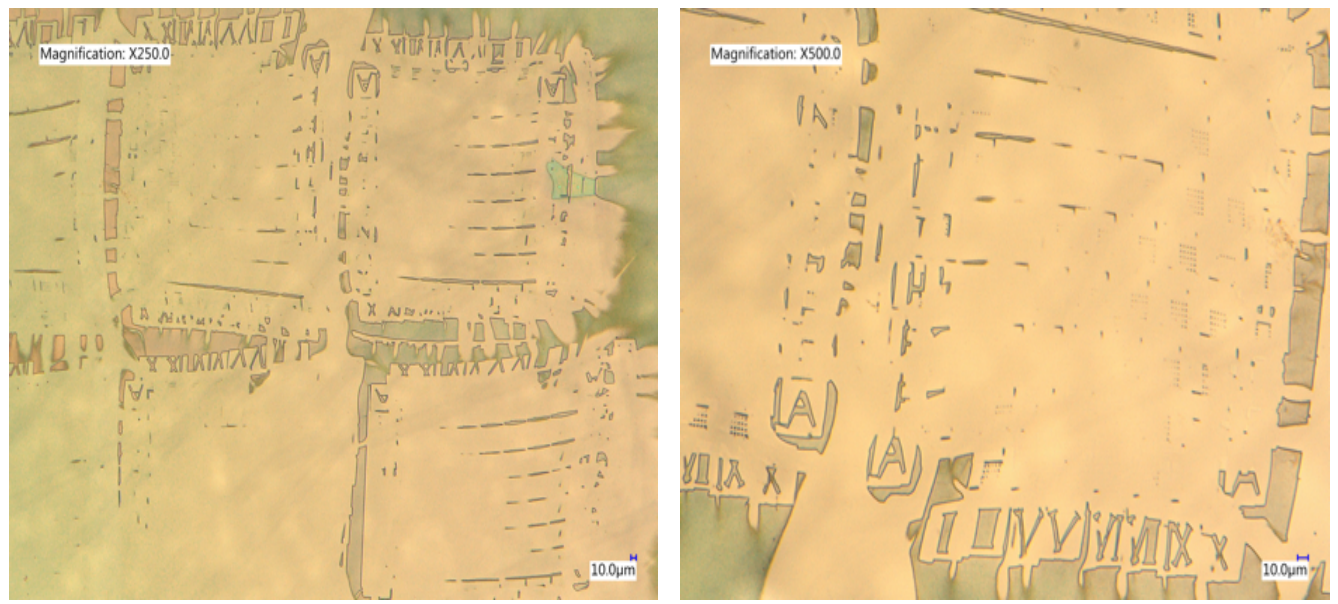

Figure 7.7: Optical microscopy image of sample (mica/PMMA/nlof2070) with exposure dose $100 \mu \mathrm{C} / \mathrm{cm}^{2}$ at different magnifications
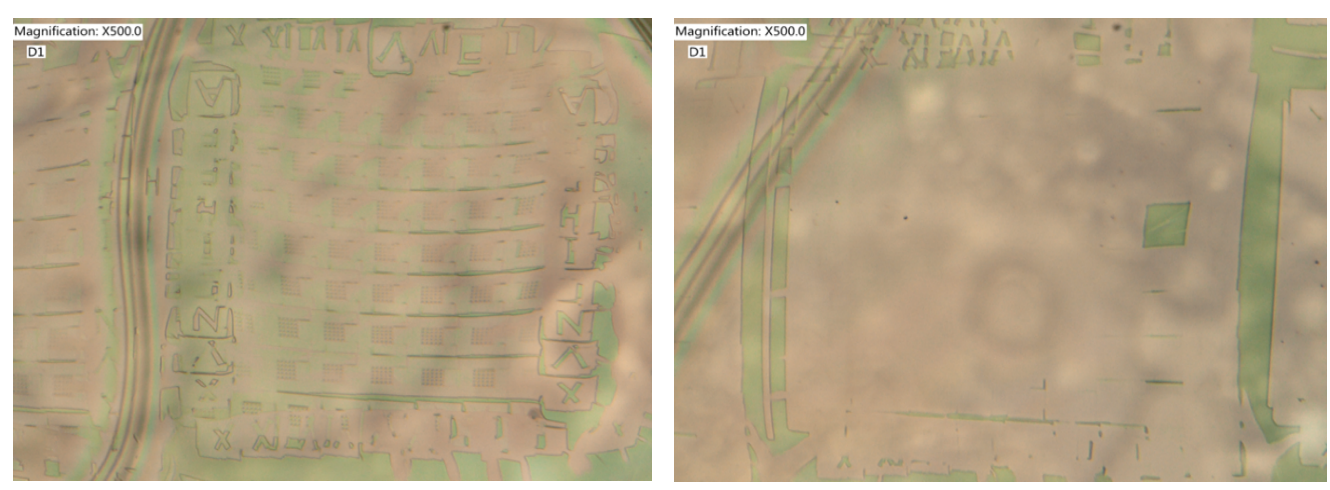

Figure 7.8: Optical microscopy image of samples (mica/PMMA/nlof2070) with exposure dose (left) 20 and (right) 50 $\mu \mathrm{C} / \mathrm{cm}^{2}$

A substrate for EBL must satisfy many requirements and be comparatively conductive. For insulating substrates, the wafer can build up an electric charge that could deform the e-beam and deflect the drawn pattern. To reduce these effects on mica, a thin metal layer, typically chromium $(\mathrm{Cr})$ or gold $(\mathrm{Au})$, can be deposited either between the substrate and the resist layer or on top of the resist [31]. This is to avoid the charging effects, which would result in the loss of patterning accuracy. For comparison purposes, the same preparation process (silicon/PMMA/nLOF 2070) and e-beam settings ( $5 \mathrm{kV}$ acceleration, $60-\mu \mathrm{m}$ aperture, dose $50 \mu \mathrm{C} / \mathrm{cm}^{2}$ ) were applied on a silicon substrate. The pattern was successfully developed on the sample (silicon/PMMA/nLOF 2070), as shown in Figure 7.9, where the details such as boxes and column header text are visible which means the substrate has been etched. 
However, the same recipe did not work when applied to the mica substrate, which confirmed that the substrate type played a role.

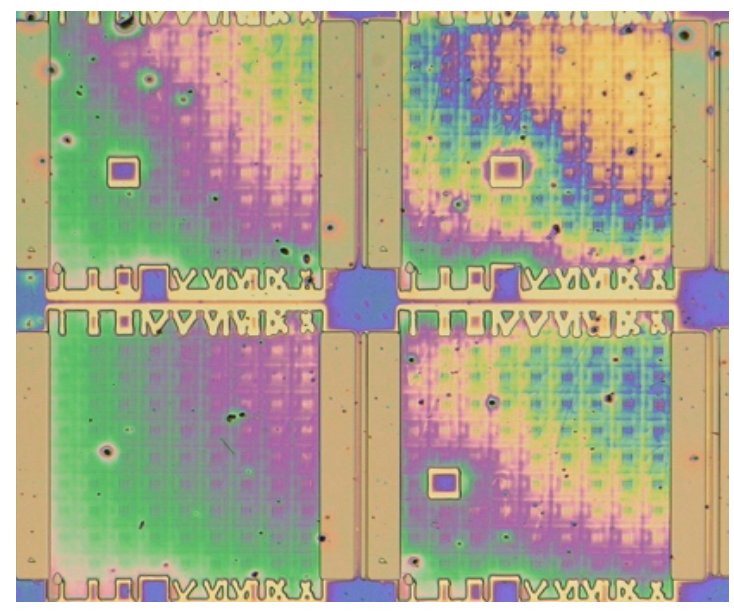

Figure 7.9: Optical microscopy image of EBL (silicon/PMMA/nLOF 2070), magnification x300

Here, the sample preparation process was repeated on the mica substrate but with the addition of a $\mathrm{Cr}$ layer. This meant the lithography recipe required several additional steps in the process, including adding the Cr layer and etching this layer. The summary of the lithography and plasma etching processes is demonstrated in Figure 7.10. First, cleaved/annealed mica substrates were coated with PMMA (stage A) and then baked at $180^{\circ} \mathrm{C}$ for two minutes; $5 \mathrm{~nm}$ of $\mathrm{Cr}$ sputter was then deposited (stage B). The negative resist deposition (stage C) was followed by pre-exposure soft baking at $100^{\circ} \mathrm{C}$ for one minute (stage D). The e-beam exposure occurred in stage E, followed by the hard bake at $110^{\circ} \mathrm{C}$ for one minute (stage F) and the developing in diluted $\mathrm{AZ} 726$ (stage G). A Cr etchant solvent was used to open the window in the Cr layer (stage H). Next, the reactive ion etcher (RIE) dry plasma was used to etch the PMMA layer (stage I), followed by the dry etching of the mica (stage J). Finally, all layers were stripped with dimethyl sulfoxide (DMSO) (stage K). 


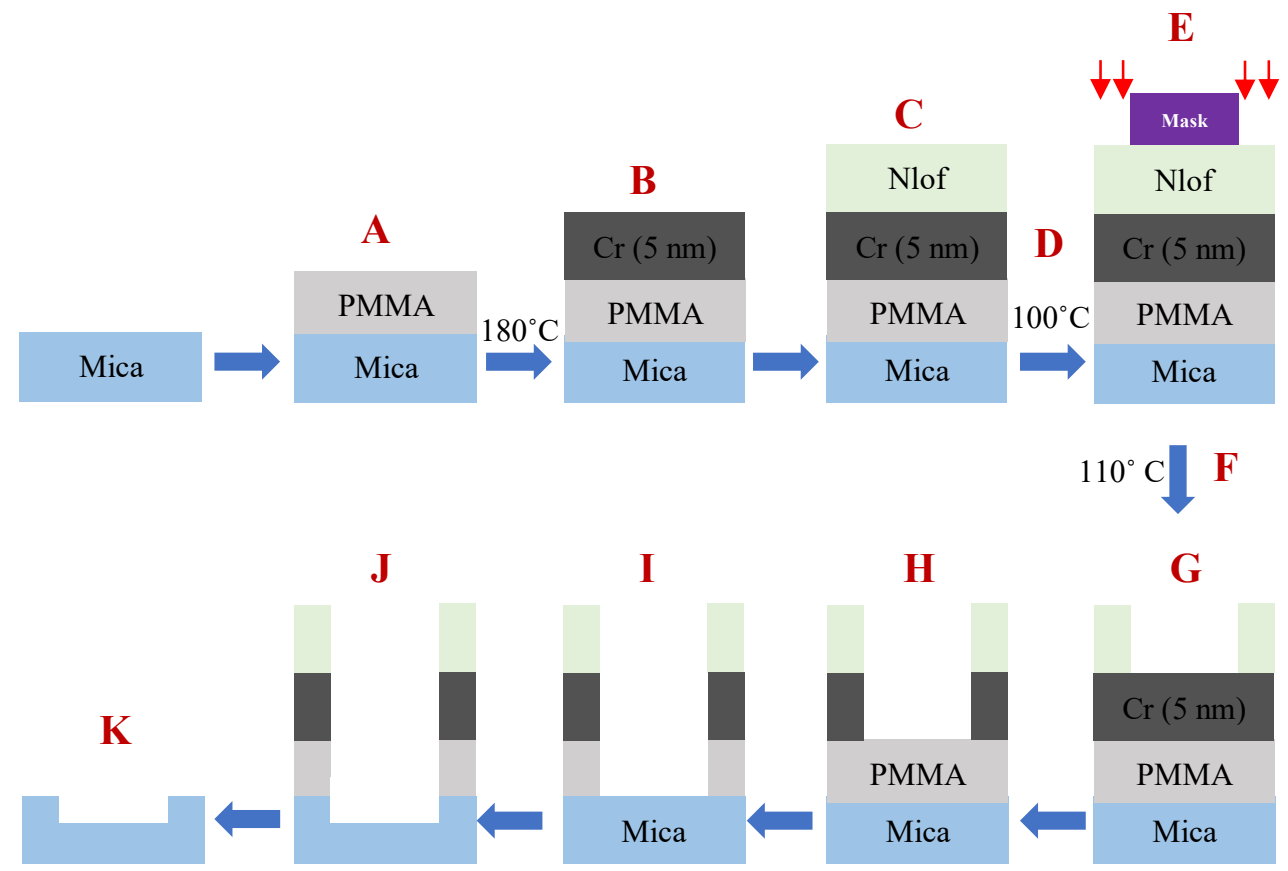

Figure 7.10: Scheme of lithography and etching process (mica/PMMA/Cr/nLOF)

After the sample (mica/PMMA/Cr/nLOF) was prepared, the e-beam exposure was tested. Two groups of exposure doses were examined. The maximum dose of S1 was $20 \mu \mathrm{C} / \mathrm{cm}^{2}$, with doses of $8,12,16$ and $20 \mu \mathrm{C} / \mathrm{cm}^{2}$. The maximum dose of S2 was 50 $\mu \mathrm{C} / \mathrm{cm}^{2}$, with doses of $20,30,40$ and $50 \mu \mathrm{C} / \mathrm{cm}^{2}$, as shown in Figure 7.11. Note: there are steps within the pattern that were produced when the mica was cleaved. These steps are not part of the pattern. One drawback of mica is that steps or terraces appear across the surface. It is not possible with EBL to select the area in which the pattern will be drawn. Although the alignment is done with electron microscopy, the beam can never be allowed to move to the main write area to image the sample, without causing premature exposure. Therefore, the alignment is performed on the edges of the substrate, and the system later patterns in the middle of the sample, even if that region contains a step. During exposure, only small patterns were drawn to reduce the exposure time, which meant that if the pattern ended up being exposed on a step, there was no other area to image. However, once a recipe is established, it is possible to repeat the pattern over the entire mica surface, where only step-free regions would be used for graphene investigation.

Figure 7.11 shown after developing for 10 seconds, the pattern was successfully drawn on the mica substrate. Then, AFM was used to measure the height of the patterned 
features, and the results indicated that the dose of $20 \mu \mathrm{C} / \mathrm{cm}^{2}$ had the deepest height and best resolution compared to the other doses. From here, it was confirmed that 20 $\mu \mathrm{C} / \mathrm{cm}^{2}$ was the optimal exposure dose for the sample (mica/PMMA/Cr/nLOF).

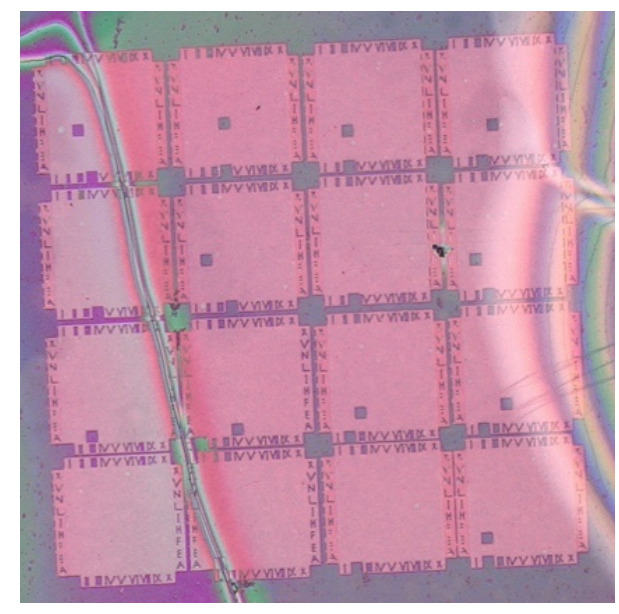

Figure

7.11:

Optical

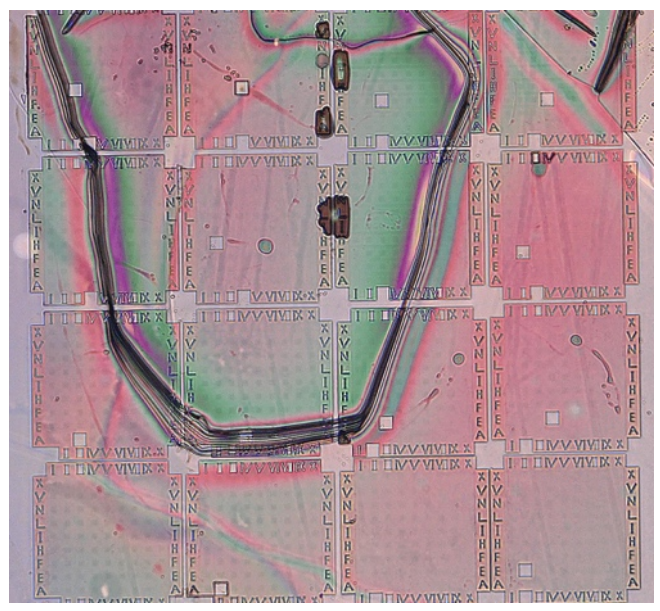

microscopy sample of mica/PMMA/Cr/nLOF. Left: S1, maximum dose of $20 \mu \mathrm{C} / \mathrm{cm}^{2}$. Right: S2, maximum dose of $50 \mu \mathrm{C} / \mathrm{cm}^{2}$. The steps within the pattern were produced when the mica was cleaved, as discussed.

\section{A2: The problem of significant erosion of the pattern caused by the developing time}

Once the pattern on the sample (mica/PMMA/Cr/nLOF) was successfully drawn by EBL, the developing time was considered. If the developing time was incorrect, the pattern would not appear in any layer; it would not develop in the nLOF, the Cr would not etch and the plasma etching would not remove any PMMA. The standard developing time for negative resist nLOF 2070 of 500-nm thickness is between 30 seconds and one minute, while in the previous sample (Figure 7.11), it was 10 seconds. Therefore, it needed to be increased by up to one minute. When the sample was developed for 30 seconds, it led to erosion in the pattern (see Figure 7.12, left). This issue worsened when the developing time was increased to one minute, as shown in Figure 7.12 (right; note the step on the pattern produced from mica cleavage). That meant that the parameter for the preparation procedure should be optimised. In the standard baking time of nLOF 2070, both the soft and hard baking times are one minute. If the baking time pre-exposure is too short, it results in a rather bad resist adhesion and a very high development rate. If the baking time post-exposure is too short, a significant erosion of the exposed resist in the developer is observed. Therefore, here, the baking time was adjusted to be two minutes instead of one for 
both the pre- and post-exposure to make the development more stable. Figure 7.13 shows the successfully developed pattern within one minute. To bring all the tested parameters together for the sample (mica/PMMA/Cr/nLOF), the baking time was two minutes, the developing time one minute and the exposure dose $20 \mu \mathrm{C} / \mathrm{cm}^{2}$. The stages from $A$ to $G$ shown in Figure 7.10 were achieved. Moving to stage $\mathrm{H}$ was done by using a $\mathrm{Cr}$ etchant solvent to etch the $\mathrm{Cr}$ layer ( $4 \mathrm{~nm} / \mathrm{second})$, which was then rinsed twice in DI water and dried with a gas gun. Then, stages I and J were carried out using plasma etching in the next section.
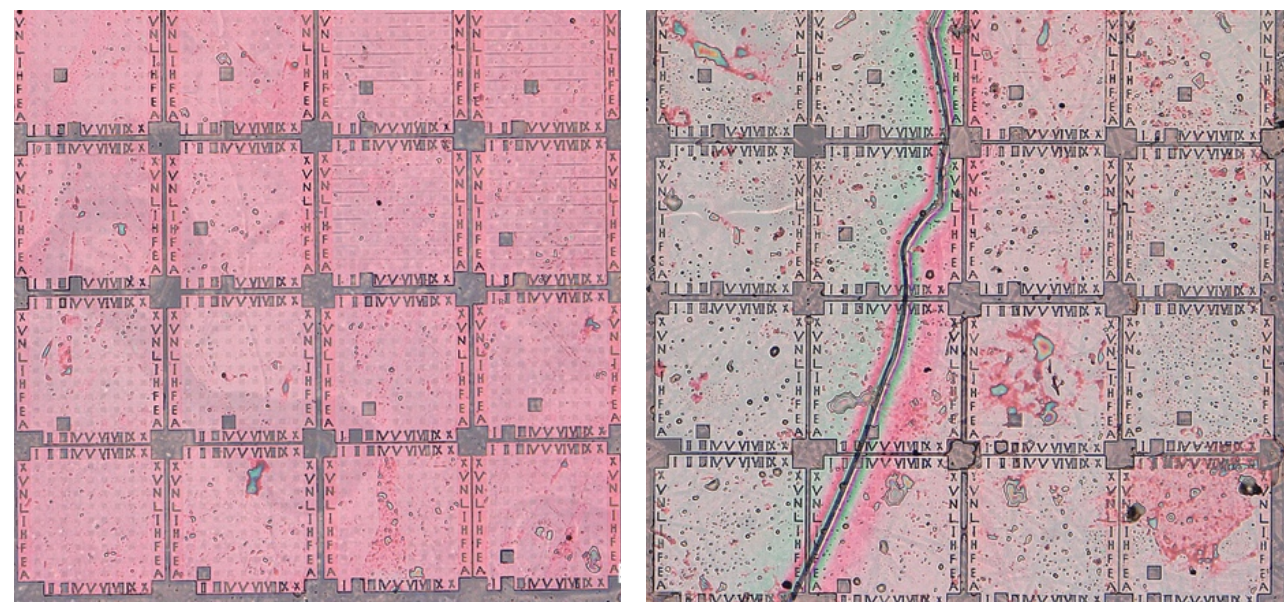

Figure 7.12: Optical microscopy image of sample (mica/PMMA/Cr/nLOF). Developing time: left: 30 s, right: 60 s. The erosion in the pattern increased with the increasing development time. Again note the step on the pattern is produced from the mica cleavage.

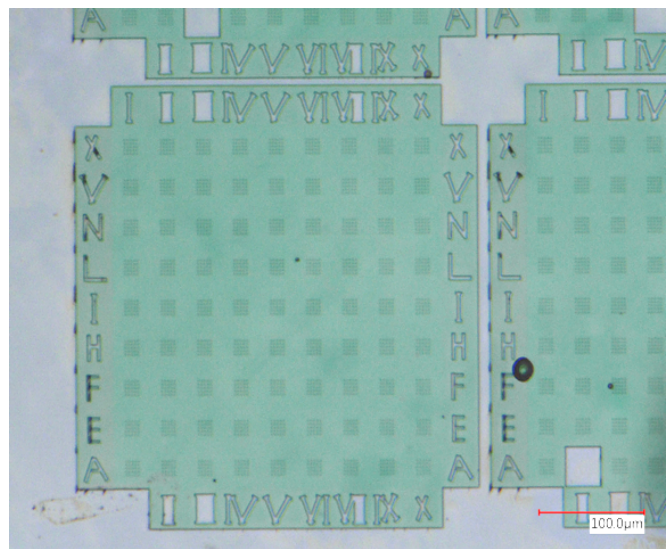

Figure 7.13: Optical microscopy image (mica/PMMA/Cr/nLOF) of baking time of two minutes for both the soft and hard bakes. Developing time: one minute for exposure dose $20 \mu \mathrm{C} / \mathrm{cm}^{2}$ 


\section{A3: The problem of not etching the PMMA layer}

Plasma etching is dependent on the process parameters, which are the radio frequency (RF) power, pressure and etching time. Each parameter needs to be optimised. After the development process, followed by the etching of the $\mathrm{Cr}$ layer using the solvent already described, plasma etching was performed. There are two steps required. First, the plasma etching must transfer the pattern in to the PMMA overlayer, and, second, the etch must continue through into the harder mica. Here, we tested a variety of these plasma etching process parameters for the sample (mica/PMMA/Cr/nLOF), but the PMMA layer initially failed to etch.

Once the sample was loaded into the chamber, the first process of etching was performed for the PMMA layer using oxygen with a flow rate of $18 \mathrm{sccm}$; the plasma was generated at an RF power of $100 \mathrm{~W}$ and a pressure of $50 \mathrm{mT}$ Torr for two minutes. This was followed by the second etching, intended to shallowly etch the mica substrate, in $\mathrm{CF}_{4}$ and an $\mathrm{Ar}$ atmosphere with a flow rate of $20 \mathrm{sccm}$ for the $\mathrm{CF}_{4}$ and 3 sccm for the $\operatorname{Ar}$ (power $100 \mathrm{~W}$, pressure 50 mTorr) for two minutes [27]. Typically, the sample was examined by an optical microscope after the dry etching process to investigate whether the pattern of PMMA/Cr/resist characteristics could be observed. If there was no pattern, this meant the plasma had been too aggressive and the layers had been removed. If the pattern was present, the next step could be carried out. After successful plasma patterning, the sample was placed in stripper DMSO D350 (MicroChemicals) in a hot $60^{\circ} \mathrm{C}$ beaker for an hour with a covered lid to remove the resist. It was then cleaned in acetone and IPA and dried with a nitrogen gun. The sample was then characterised by optical microscopy, and if there was no pattern, the RIE plasma etching recipe had failed to etch the mica. In this work, the pattern was observed by optical microscopy after the dry etching, but the pattern was not present after stripping the layers using the DMSO D350 solvent. This implied that the PMMA was still not etched properly yet and that the plasma had not reached the mica surface.

Thus, this plasma recipe was adjusted to pattern the PMMA layer. First, $\mathrm{O}_{2}$ plasma was used as a 'window opening' step; a low-pressure process in the range of 5 to 20 mTorr was used, and the power was reduced to between 30 and $40 \mathrm{~W}$ to get better control of the PMMA etching. The oxygen flow was increased from 5 to $15 \mathrm{sccm}$, within range of the etching time of between two and six minutes. All these recipes failed to pattern the PMMA, and, subsequently, the mica layer was unsuccessfully 
etched. Although the Cr layer was no more than half the thickness of the PMMA, it was still difficult to etch the PMMA layer. Typically, PMMA has poor strength resistance to plasma etching, but, in this work, it had good resistance. It could have been that the annealing step in the final few stages changed the PMMA's properties, as baking is one of the solutions that other studies have used to improve the poor etching selectivity of PMMA.

\section{B: Ultraviolet (UV) photolithography process steps for the second recipe}

As there were issues in etching the PMMA layer, the experiment was repeated, replacing EBL with UV lithography. Photolithography is a much faster technique to rapidly expose and test more process parameters to obtain an optimal recipe that could later applied on EBL. Here, the PMMA layer was not used in the preparation recipe because of the problems with etching described above; in this case, the Cr layer was used as a mask to protect the mica surface.

As shown in Figure 7.14, mica disk substrates were cleaved and annealed. A Cr layer was deposited on top of the substrate; however, the $\mathrm{Cr}$ layer was adjusted to be thicker than $5 \mathrm{~nm}$ (different thicknesses were tested later) in order to be as a hard mask to protect the mica from the plasma etching process and the resist developer solvent. In addition, without the hard mask, the pattern could not survive with high plasma power either. Here, the $\mathrm{Cr}$ layer was chosen to be on top of the mica rather than the photoresist (PR), as the order of this sample preparation recipe could not be reversed for two reasons. First, the developer solvent could etch the mica; therefore, there had to be a layer between the mica and the PR. Second, the negative resist AZ nLOF 2070 is sensitive to white light, and, therefore, it had to be deposited after the Cr layer. Next, the $\mathrm{AZ}$ nLOF 2070 was deposited, followed by soft baking at $100^{\circ} \mathrm{C}$ for two minutes and then UV exposure at $25 \mathrm{~mW} / \mathrm{cm}^{2}$. After that was the PEB at $110^{\circ} \mathrm{C}$ for two minutes; then, the sample was left for five minutes to hydrate. Following that, the sample (mica/Cr/nLOF) was developed in diluted 2:1 AZ 726 MIF:DI water for one minute, followed by a lower concentration of the developer, and was then rinsed with DI and dried, as mentioned previously. The next step was to leave the sample in $\mathrm{Cr}$ etchant solvent for an elevated amount of time (4 $\mathrm{nm} / \mathrm{second})$ depending on the thickness of the Cr; then, it was rinsed twice in DI water and dried. Then, plasma etching, resist stripping (different type of strippers were tested later) and Cr stripping were performed. However, these three stages were further optimised in 
correspondence with the issues in the next section to overcome the difficulties of removing the resist layer.

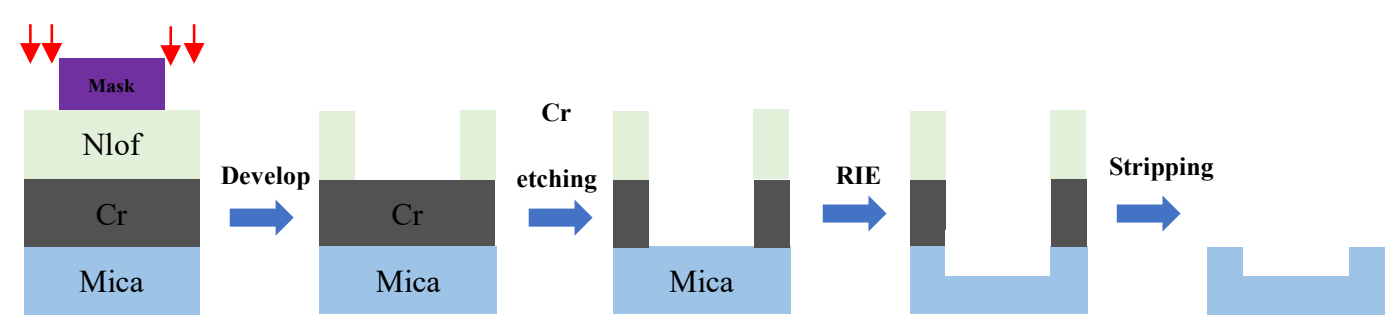

Figure 7.14: Scheme of patterning process of mica $/ \mathrm{Cr} /$ negative resist nLOF 2070 using UV exposure.

\section{B1: The problem of the resist hardness after RIE}

Here, cleaved annealed mica was coated in a 25-nm-thick Cr layer; then, the PR was deposited on the sample. After that was the processing of the photolithography, including UV exposure, developing and $\mathrm{Cr}$ etching, as mentioned above. After cleaning the RIE chamber, $\mathrm{CF}_{4}$ and Ar gases with a flow rate of $40 \mathrm{sccm}$ and $10 \mathrm{sccm}$, respectively, were applied under RF power $150 \mathrm{~W}$ with pressure $50 \mathrm{mTorr}$ for two minutes and then optical microscopy was used to check if the pattern was still present. If it was, the next step was carried out: the stripper DMSO was used for one hour to remove the remaining PR and then strip the $\mathrm{Cr}$ layers to detect whether the mica surface had been etched or not. As in Figure 7.15 (left), the pattern was still present after the plasma etching. However, DMSO was unable to strip the PR layer, even after four hours. This could have been due to the fact that the plasma affected the PR much more, as shown in Figure 7.15 (right). Therefore, stronger strippers, including types P1316 and NI555, were tried but caused peeling on the mica surface itself. It is very important to strip PR correctly to pattern the mica surface. Thus, in the next experiment, the order of the stages was changed by stripping the PR before plasma etching. In this case, only the $\mathrm{Cr}$ layer on top of the mica were remained while the resist was removed completely before the plasma will performed. As shown in Figure 7.16 (left), the pattern after an hour in the stripper (right) was clean, and no resist layer remained after the plasma etching. Thus, from here, the sample (mica/Cr/nLOF 2070) was developed, followed by etching the $\mathrm{Cr}$ layer using the solvent and then stripping 
the PR before plasma etching to avoid hardness in the PR layer caused by the dry plasma.
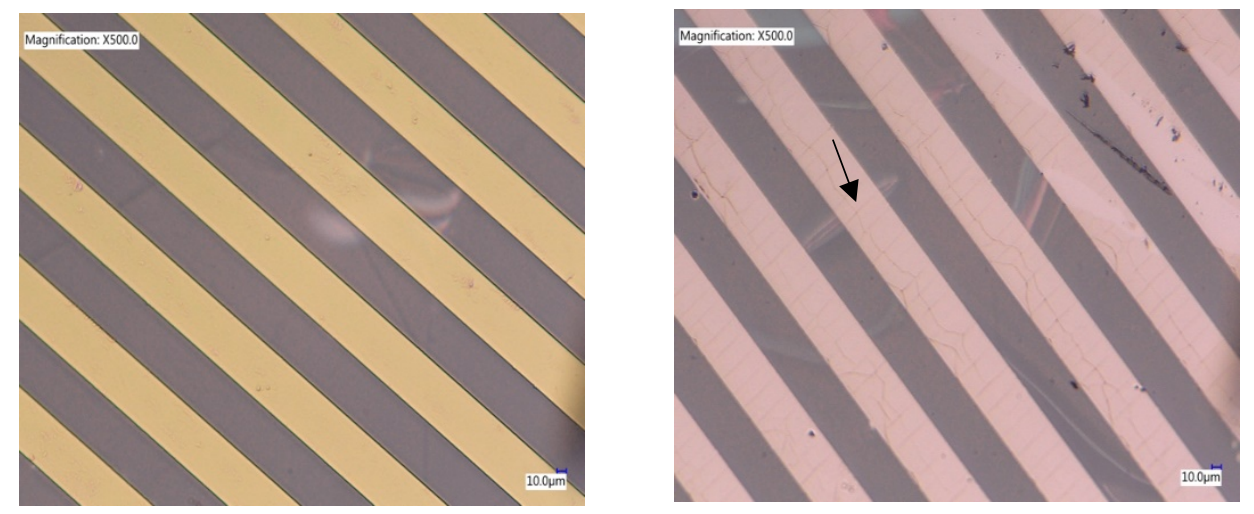

Figure 7.15: Optical microscopy image of mica/Cr/resist. Left: mica after dry plasma stage. Right: mica after PR stripper DMSO for four hours
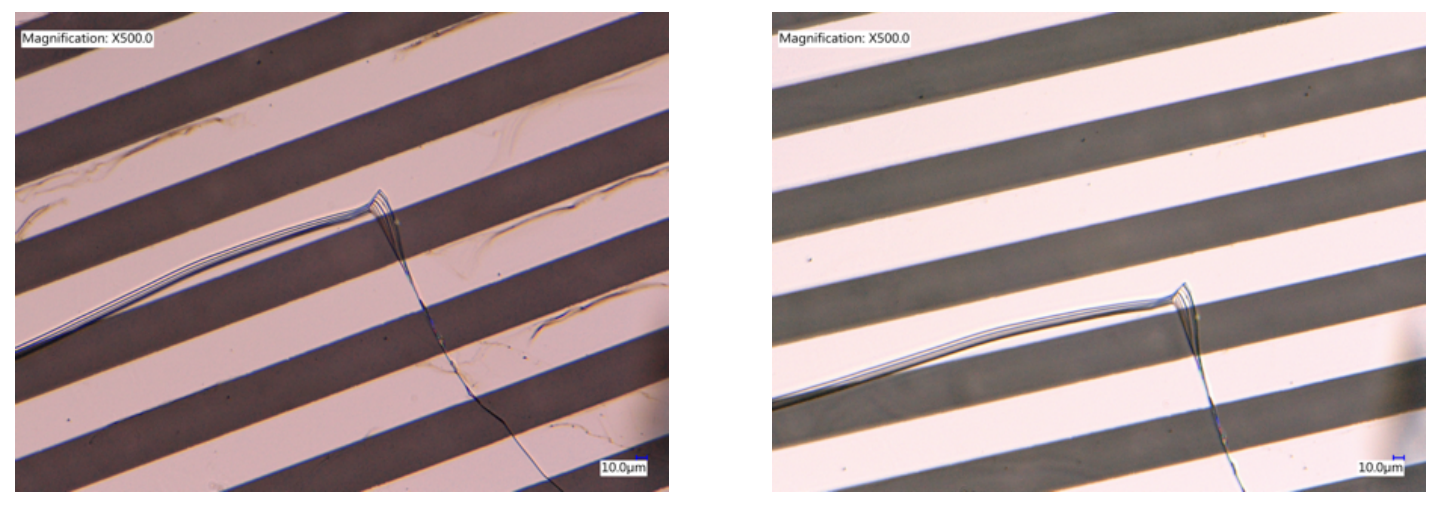

Figure 7.16: Optical microscopy image of mica/Cr/resist Left: mica after stripper for one hour. Right: mica after dry etching.

\section{B2: The problem of shallow etching mica}

Shallow etching of the mica substrate in the sample (mica/Cr/nLOF 2070) was another problem that required a solution. After the UV photolithography process was completed, including the exposure, developing, $\mathrm{Cr}$ etching and resist stripping as mentioned above, the plasma etching process was performed with $40 \mathrm{sccm}$ of $\mathrm{CF}_{4}$ and 
$10 \mathrm{sccm}$ of Ar by applying a power of $150 \mathrm{~W}$ and pressure of $50 \mathrm{mTorr}$. After the dry etching, whether or not the pattern was still present had to be checked, as now the $\mathrm{Cr}$ layer was exposed to the plasma without any PR layer. From the previous experiment with an etching time of two minutes, the Cr layer was still visible, meaning that the plasma had not removed the $\mathrm{Cr}$ layer, and the etching time could be increased to five minutes. The purpose of increasing the time gradually and testing if the $\mathrm{Cr}$ layer was still present was to ensure that the $\mathrm{Cr}$ was not being etched by the RIE. If the plasma was too aggressive, the Cr layer would be etched but the mica would not be. Figure 7.17 shows the process of photolithography and mica etching, starting with a) after developing the pattern and Cr etching; b) stripping the PR using DMSO for one hour; c) after dry etching for five minutes; and, finally, d) after the Cr layer was stripped to eventually show the etched mica surface. Note mica cleavage caused the steps on the pattern. Figure 7.18 shows images of the sample $(\mathrm{mica} / \mathrm{Cr} / \mathrm{resist})$ and (left) the scanning electron microscopy (SEM) for a horizontal line of sidewall in very shallowly etched mica. Figure 7.18 (right) shows the optical microscopy image for the same sample; the dashed line shows an example of a sidewall-etched pattern in the mica. The pattern is not fully clear in the optical image due to the transparency of the mica substrate, and it is difficult to organise the shallowly etched features. Note that it is the same area as in Figure 7.17 (d) but with higher magnification. However, to improve the depth of the etched mica, the plasma settings were adjusted in the next experiment. 


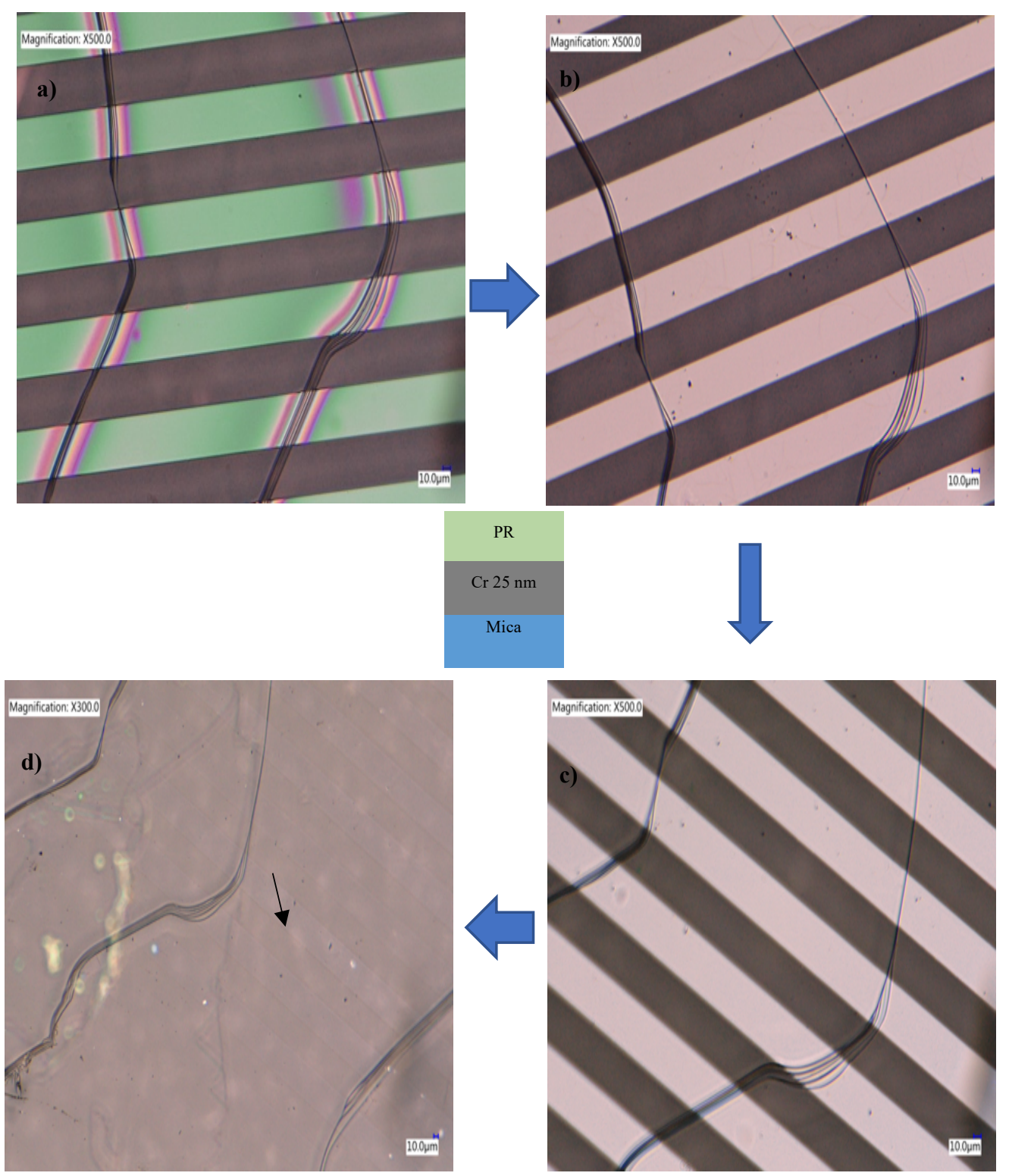

Figure 7.17: Optical microscopy image (mica/Cr/nLOF 2070) of a) after development and $\mathrm{Cr}$ etching, b) after PR stripping, c) after plasma etching, and d) after stripping the Cr layer. The steps on the pattern produced from mica cleavage 

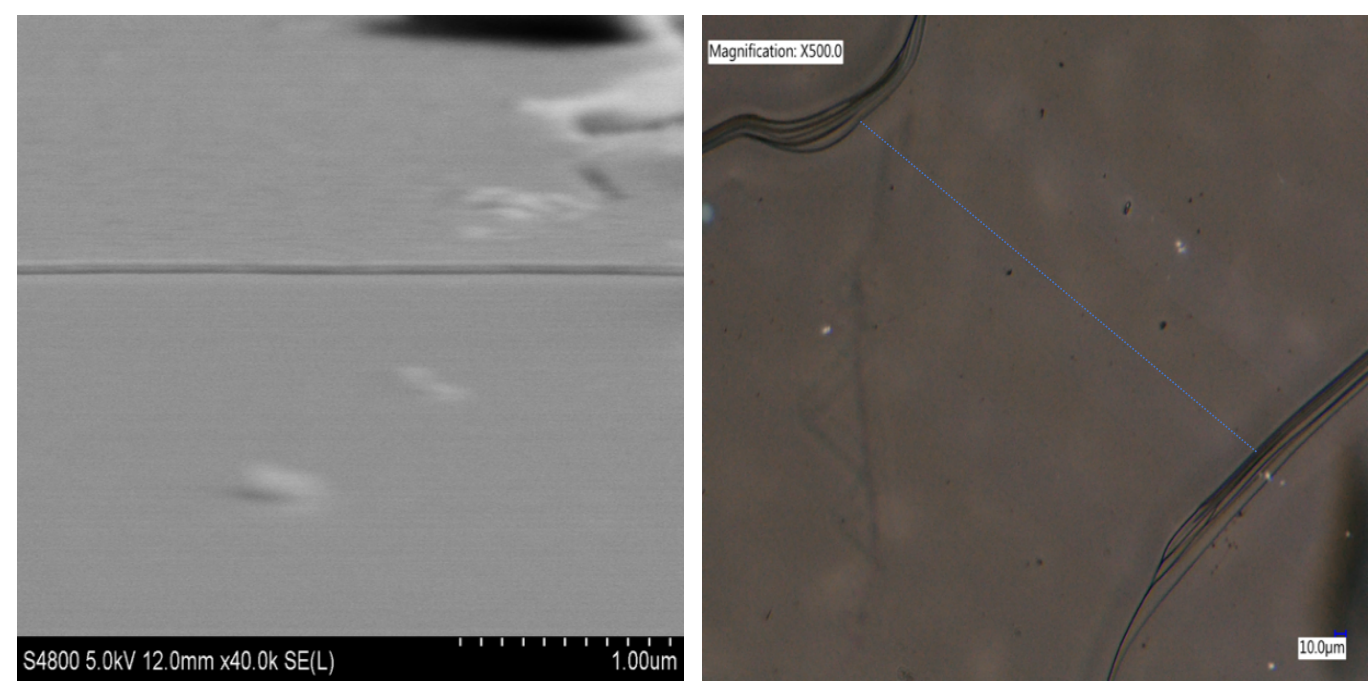

Figure 7.18: (Left) SEM image of shallow-etched mica surface prepared by mica/ $25 \mathrm{~nm} \mathrm{Cr} / \mathrm{nLOF} 2070$ ). The upper part of the image is unetched, the horizontal line is the sidewall, and below that the trench of the etched line. (Right) The same sample with optical microscopy; the dashed line shows the orientation of the faint stripes, which are the repeated stripes just visible. The curved lines are cleave-induced steps.

\section{B3: Optimisation of RIE parameters}

As the mica had now been etched by a few nanometres, the plasma parameters needed to be increased, as did the thickness of the Cr layer (from 25 to $70 \mathrm{~nm}$ ). This was to ensure that the $\mathrm{Cr}$ layer was not etched completely and that the mask was not lost. The flow rate of the gases was $\mathrm{CF}_{4}$ at $50 \mathrm{sccm}$ and Ar at $20 \mathrm{sccm}$; the power was $200 \mathrm{~W}$, the pressure was 100 mTorr and the etching time was two minutes. The AFM measurements were taken after the stage of photolithography, after the etching and after the Cr stripping to demonstrate the etch rate of the mica. The height of the patterned sample before the dry plasma was around $69 \mathrm{~nm}$. After the RIE, the height was around $60 \mathrm{~nm}$, and after the Cr layer was stripped, the depth was $10 \mathrm{~nm}$ for the mica surface. This indicated a 7:1 etch ratio for Cr:mica, which was expected because of the hardness of mica and its resistance to plasma etching. Figure 7.19 shows the optical image of the mica/70 nm Cr/nLOF 2070 (left) after plasma etching and (right) the etched mica substrate after the stripping of all the coated layers; the dashed line is 
to guide the eye, as the pattern is not fully clear in the optical image because of the transparency of the mica substrate.
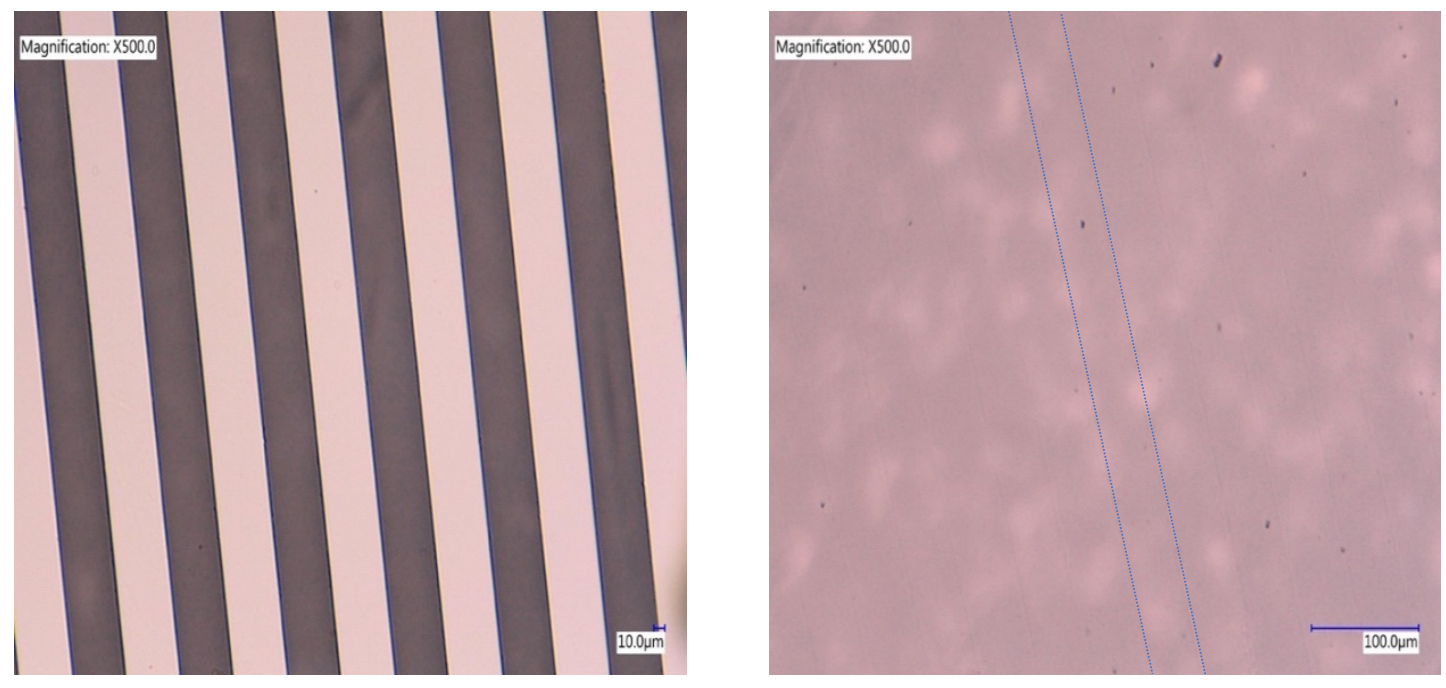

Figure 7.19: Optical microscopy image of mica/70 nm $\mathrm{Cr} / \mathrm{nLOF}$ 2070. Left: the pattern after dry etching. Right: the etched mica of $10 \mathrm{~nm}$ after stripping of all the coated layers. The dashed line shows an example to guide the eye to an etched trench in the mica

Then, the same procedure from the previous experiment on the mica/70 nm $\mathrm{Cr} / \mathrm{nLOF}$ 2070 was used but the thickness of the $\mathrm{Cr}$ layer needed to be increased to subsequently increase the etching time to increase the etch depth for the mica. Thus, all the parameters for the dry etching could remain the same as above (200 W power, 100 mTorr pressure, $50 \mathrm{sccm} \mathrm{CF}_{4}$ and $20 \mathrm{sccm}$ Ar), with the only change being an increase in the etch time of up to 10 minutes. As a result of finding the $\mathrm{Cr}$ : mica etch ratio of $\sim 7: 1$, the thickness of the Cr layer was increased by depositing $250 \mathrm{~nm}$ instead of 70 $\mathrm{nm}$. After the RIE, the height of the sample was around $270 \mathrm{~nm}$ as shown in figure 7.20, including the depth of the Cr layer and etched mica. After the Cr layer was stripped, the optical image showed successfully etched mica substrate. AFM confirmed the depth of the etched mica substrate as $40 \mathrm{~nm}$ in Figure 7.21. However, comparing with Figure 7.19 (right) the etched vertical stripe had to be indicated by overlain lines, but here the etch is deep enough to provide clear optical contrast, directly confirming the etch depth increase in advance of AFM profiling. Eventually, 
it was possible to repeat this method using EBL as the mica had been etched successfully with a remarkable depth.
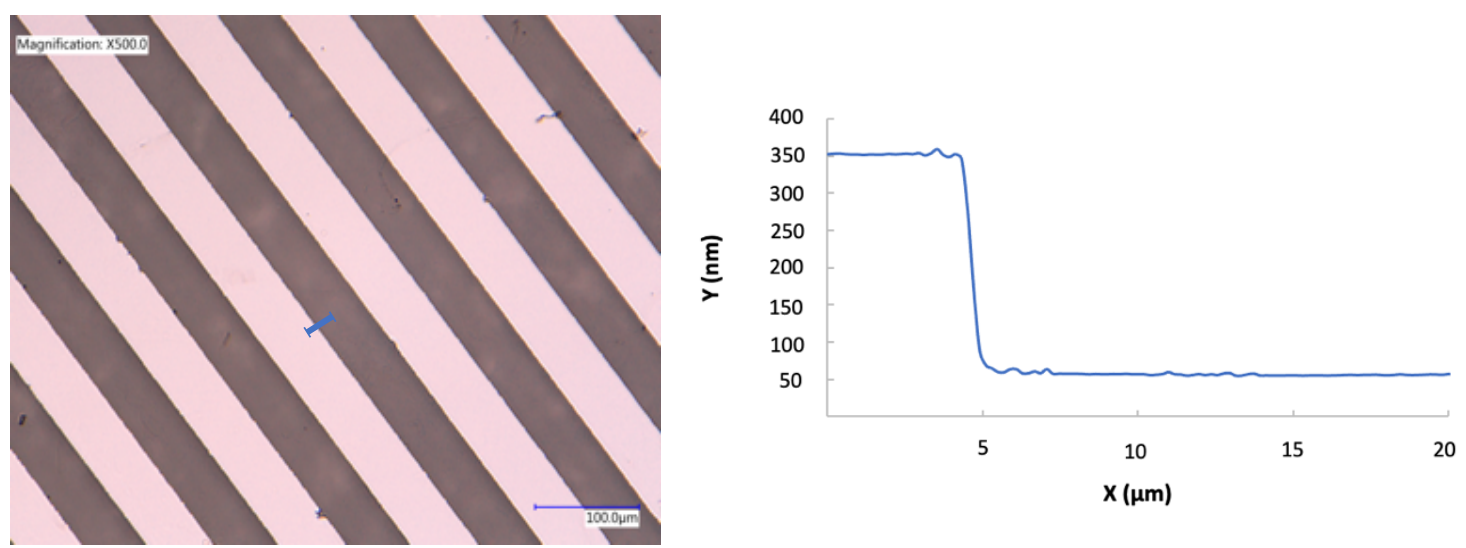

Figure 7. 20: Left: Optical image of (mica/250 nm Cr/nLOF) after RIE.

Right) AFM measurement of the etched mica corresponding to the line scan indicated on the optical image
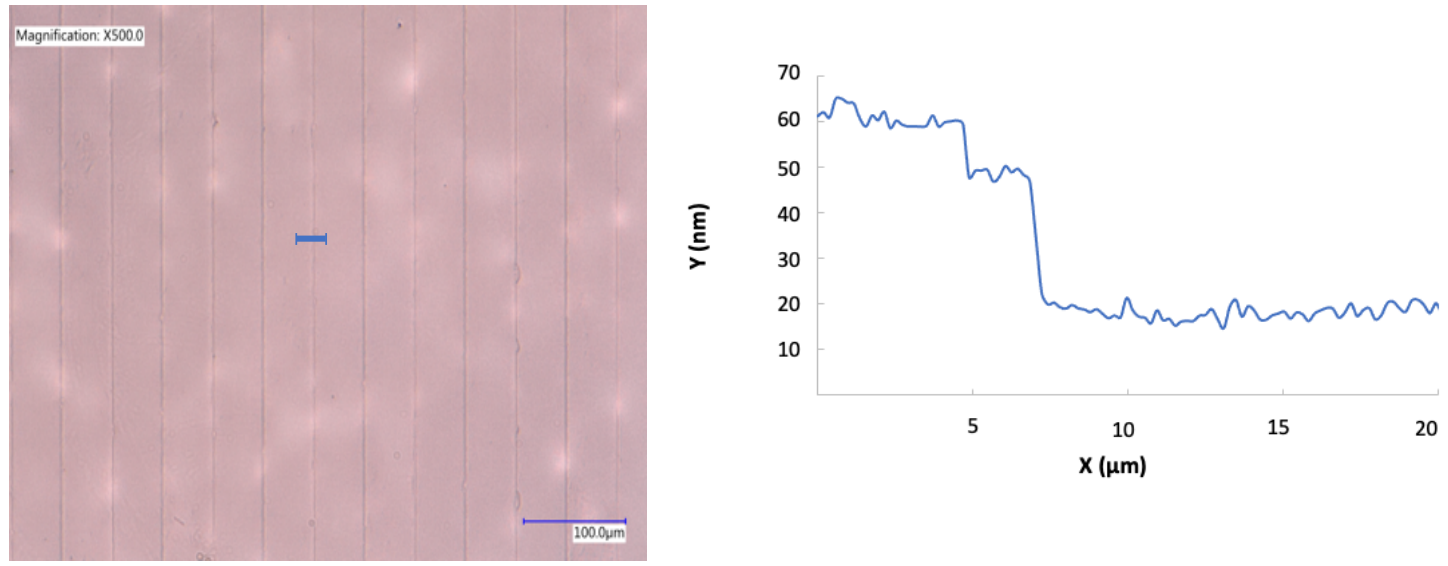

Figure 7.21: Left) Optical microscopy image (mica/250 nm Cr/nLOF) of etched mica substrate. The increased depth provides more optical contrast. Right) AFM profile of the etched mica as the line scan ion the optical image. 


\section{C: Final lithography process}

Here, the lithography procedure repeated the same method as the successful UV photolithography process. It started with a cleaved/annealed mica disk; a thick layer $(\sim 250 \mathrm{~nm})$ of $\mathrm{Cr}$ was deposited on top of the substrate. Then, a negative resist e-beam AZ nLOF 2070 was applied to the sample and spun at 4,000 rpm for 40 seconds, followed by a soft bake at $100^{\circ} \mathrm{C}$ for two minutes. After that, the sample exposure was performed at $5 \mathrm{kV}$ acceleration, a $60-\mu \mathrm{m}$ aperture and a beam current of around 0.32 $\mathrm{nA}$ with a maximum dose of $50 \mu \mathrm{C} / \mathrm{cm}^{2}$ to examine the depth of mica etching under different exposure doses. After the $\mathrm{PEB}$ at $110^{\circ} \mathrm{C}$ for two minutes, the sample was left for five minutes to hydrate. Next, the development was performed with a concentration of the diluted developer in 2:1 AZ 726 MIF: DI water for one minute, followed by 1:1 and 1:2 diluted developer. Finally, the sample was rinsed quickly in pure DI water and then dried with a nitrogen gun.

The next step was to etch the Cr layer using solvent and then rinse it twice in DI water and dry it with $\mathrm{N}_{2}$ gas. The resist was stripped for an hour in hot DMSO. It was then cleaned with acetone and IPA and dried with a $\mathrm{N}_{2}$ gun. Then, dry etching was performed with $\mathrm{CF}_{4}(50 \mathrm{sccm}), \operatorname{Ar}(20 \mathrm{sccm}), 200 \mathrm{~W}$ power, $100 \mathrm{mTorr}$ pressure and an etching time of 10 minutes. Finally, the $\mathrm{Cr}$ stripper removed the remaining $\mathrm{Cr}$ layer, and it was rinsed twice in DI water and dried with $\mathrm{N}_{2}$ gas. Figure 7.22 shows most of these stages, starting with a) drawing the pattern in the software (the maximum exposure dose was $50 \mu \mathrm{C} / \mathrm{cm}^{2}$ including 40,30 and $20 \mu \mathrm{C} / \mathrm{cm}^{2}$ ). The next stage was developing for b) 30 seconds, while the best developing time was one minute, shown in c). After the Cr etching and resist stripping, dry plasma was performed as shown in d). Finally, the stripped $\mathrm{Cr}$ and etched patterned mica substrate was obtained; e) and f) presented different etched areas. Note that the centre of the patterns always shows the best result as the coated layers, such as the resist and Cr layers, may not have deposited uniformly over the whole surface; thus, $\mathrm{Cr}$ was still remaining in some areas. To examine the best exposure dose, AFM was performed. Figure 7.23 shows the AFM images for different exposure doses in the range of 20 to $50 \mu \mathrm{C} / \mathrm{cm}^{2}$. The pattern in these doses showed a nearly similar depth, but among them, the exposure dose of 40 $\mu \mathrm{C} / \mathrm{cm}^{2}$ provided the deepest holes in the mica substrate of $35 \mathrm{~nm}$ as shown in AFM profile in Figure 7.24. 

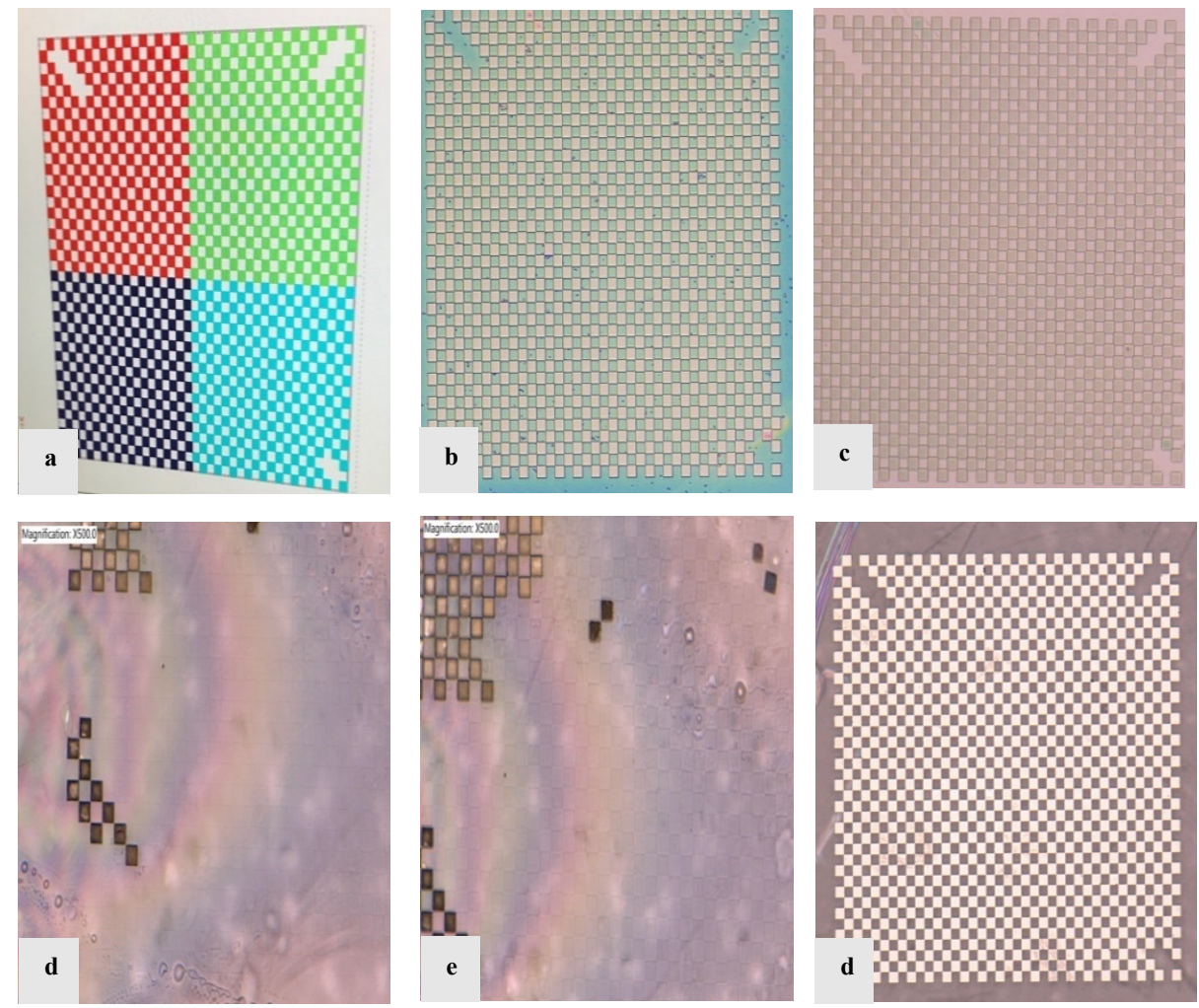

Figure 7.22: Optical microscopy image of patterning EBL for mica substrate. Sample of mica/250 nm Cr/nLOF 2070. a) Pattern drawn with software (dose of dark blue is 20 , light blue is 30 , green is 40 , red is 50 , $\mu \mathrm{C} / \mathrm{cm}^{2}$ ). b) After developing for 30 seconds. c) After developing for one minute. d) Post RIE. e) and f) Different areas of etched mica after stripping of all the layers 

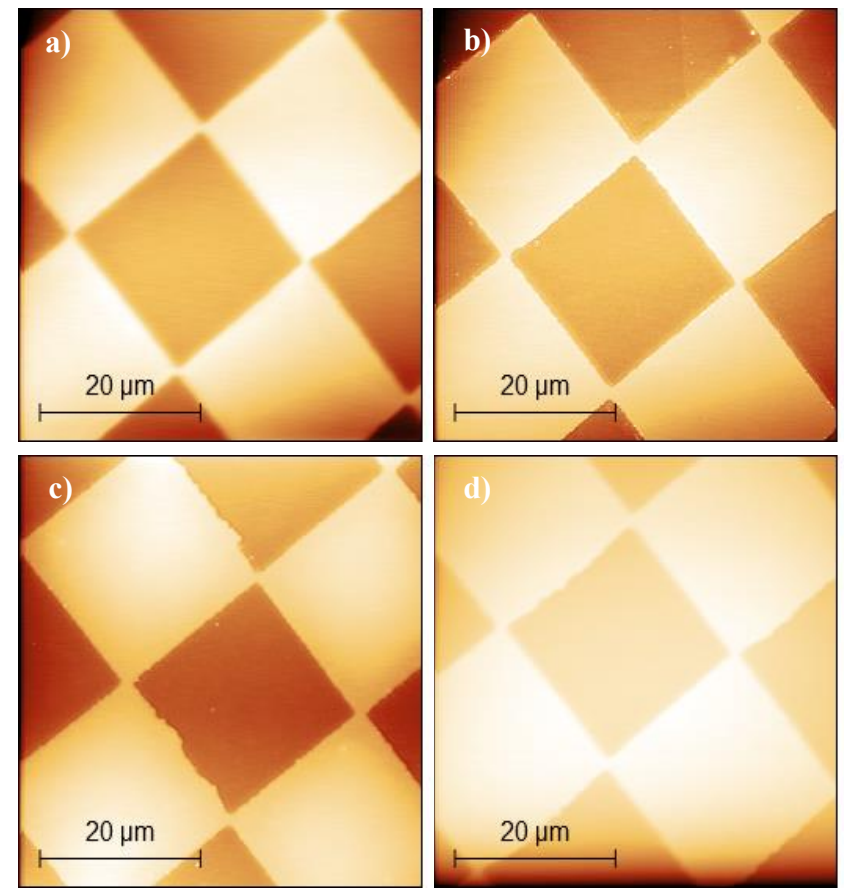

Figure 7.23: AFM images for mica patterned at different exposure doses a) 20, b) 30 , c) 40 and d) $50 \mu \mathrm{C} / \mathrm{cm}^{2}$
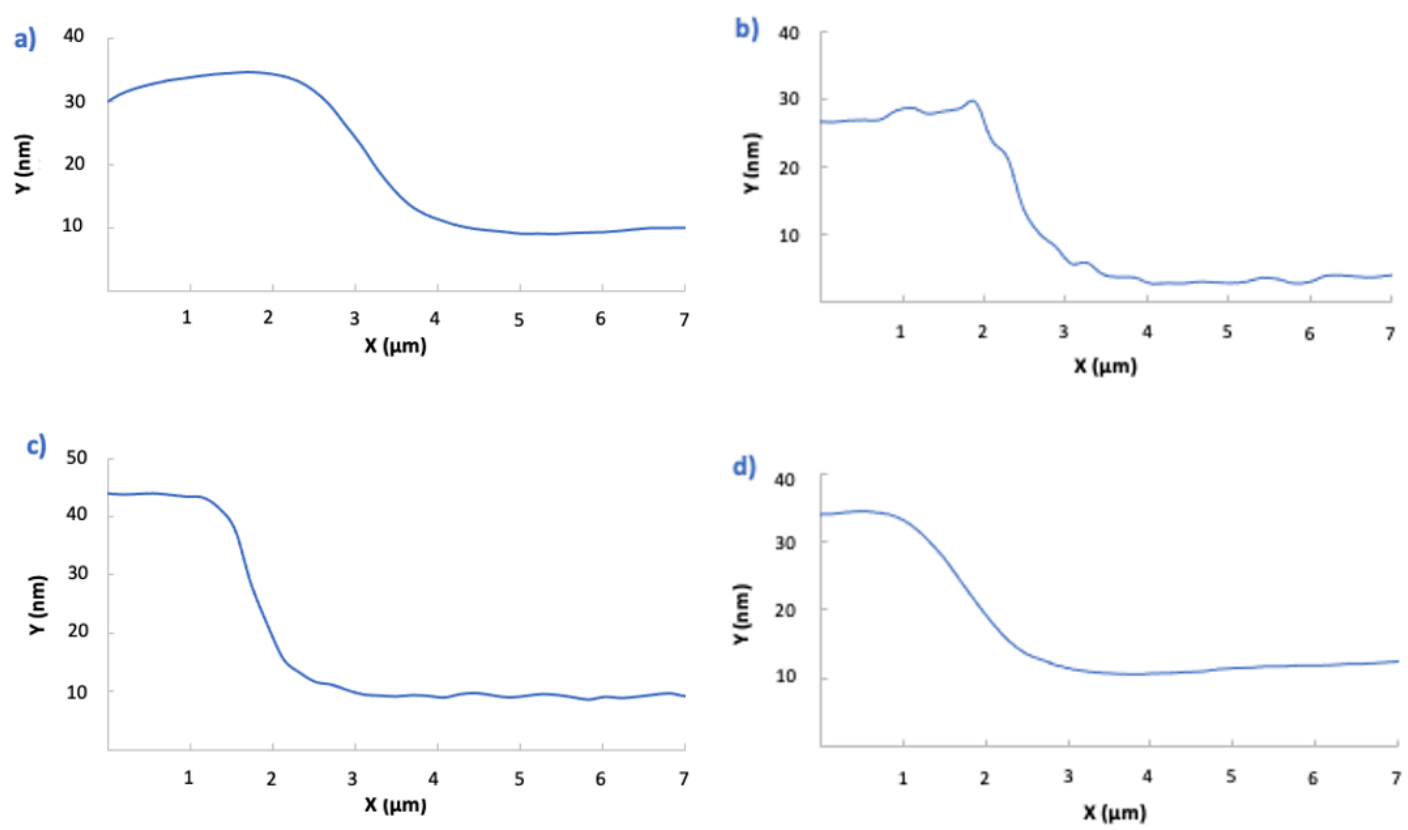

Figure 7. 24:AFM profile for mica patterned at different exposure doses a) 20 , b) 30 , c) 40 and d) $50 \mu \mathrm{C} / \mathrm{cm}^{2}$ 
Figure 7.25 shows the optical image for another example of patterned mica with exposure to dose $40 \mu \mathrm{C} / \mathrm{cm}^{2}$ at different magnifications. The boundary of the etched pattern is indicated with the arrow, and are straight as intended. The curvy lines are cleavage-induced steps. In addition, AFM showed a hole size of $2 \mu \mathrm{m} \times 2 \mu \mathrm{m}$ with 1 $\mu \mathrm{m}$ separation. The depth of the hole for this sample was indicated as $35 \mathrm{~nm}$ deep, as shown in Figure 7.26.

Finally, the mica substrate was etched successfully. Typically, researchers etch mica substrate using the hazardous solvent of HF acid, but, here, in this work, the mica surface was etched with none of this solvent and the pattern was controlled by EBL without using a physical mask such as TEM grids or UV masks.
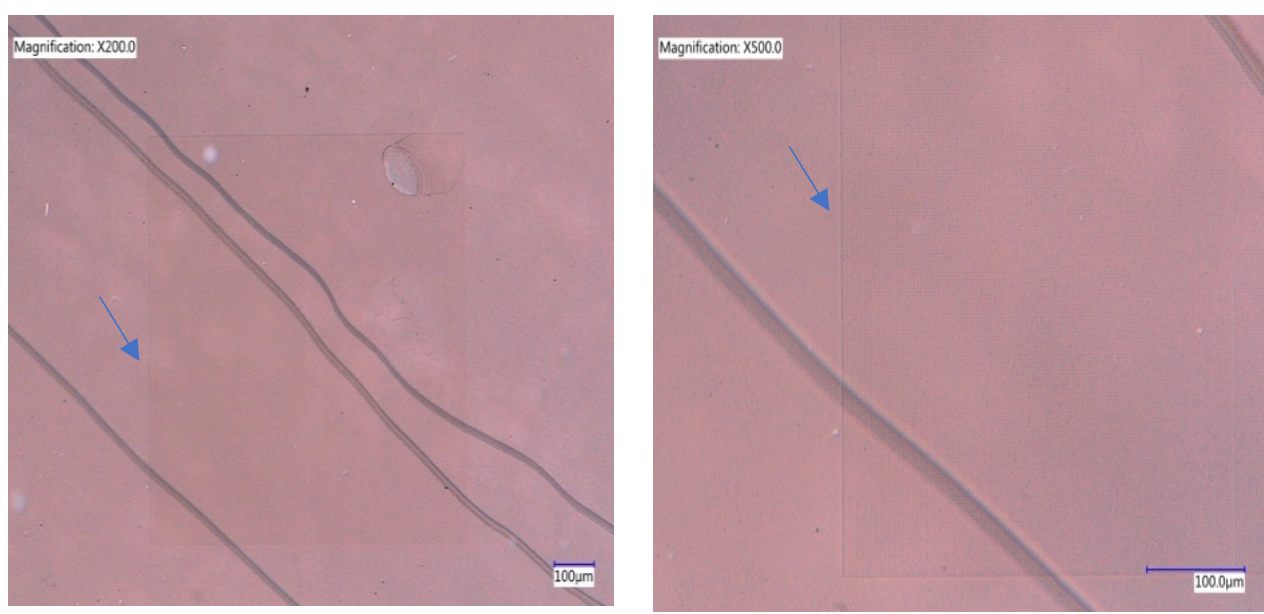

Figure 7.25: Optical image of patterned mica substrate at different magnifications, indicated with arrows as the fainter lines. Note, the wavy steps on the pattern are produced from mica cleavage. 

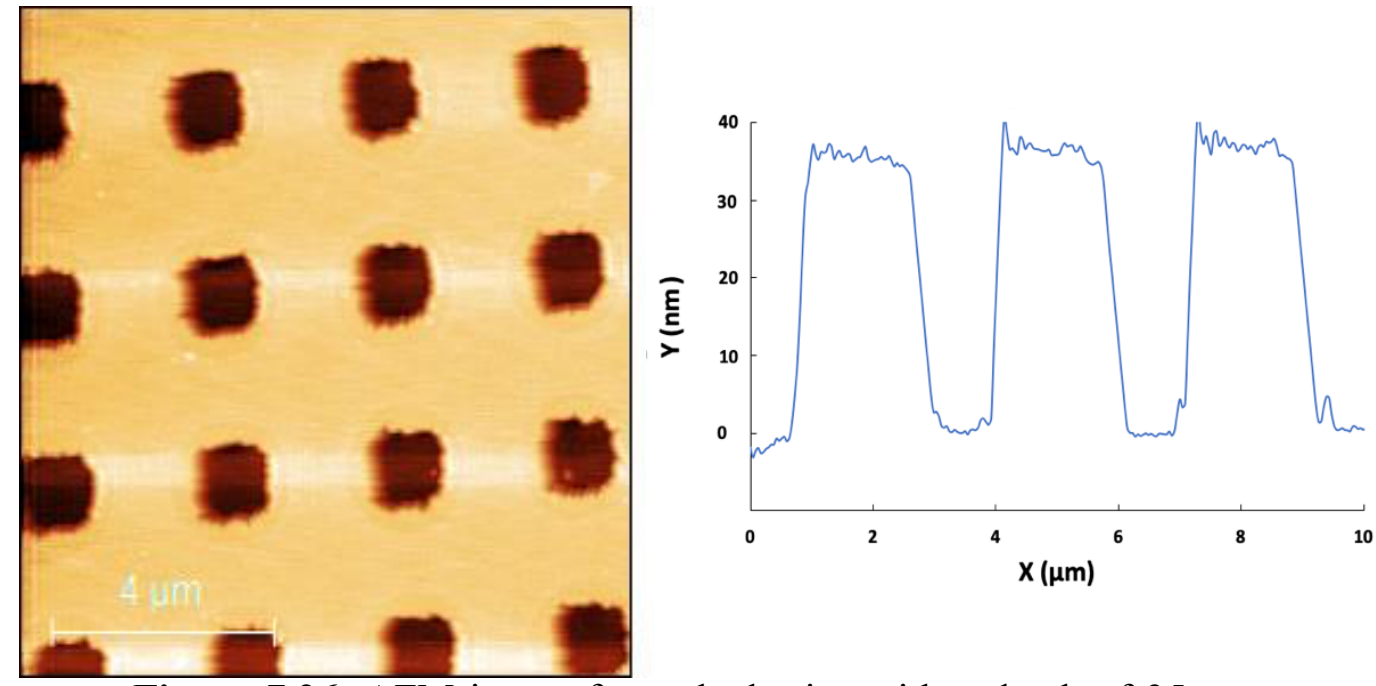

Figure 7.26: AFM image for etched mica with a depth of $35 \mathrm{~nm}$. Sample of mica/250 nm Cr/nLOF 2070. Exposure dose $40 \mu \mathrm{C} / \mathrm{cm}^{2}$

\subsubsection{Suspended graphene on mica substrate}

The goal of this entire thesis was to be able to pattern mica using EBL and then deposit graphene. Therefore, the final step was to check whether it was possible to mechanically exfoliate graphene onto the now successfully etched substrates.

After the sample was patterned with EBL and plasma etching, the patterned mica substrate was annealed at $200^{\circ} \mathrm{C}$ for two minutes to remove any layers of contamination. The mechanically exfoliated graphene was transferred to mica, as described in Chapter 4, using dicing tape. The transfer was successfully achieved and was imaged using a number of techniques to confirm the transfer. Figure 7.27 shows the same suspended graphene on patterned mica using optical microscopy and SEM techniques. The optical microscopy settings were optimised to clearly image the graphene sheet on the patterned mica substrate, while SEM showed better contrast to image the same sheet. The AFM technique presented more information, as shown in Figure 7.28, for another sample of the suspended graphene sheet that was deposited on the clean patterned mica surface. However, the patterns were achieved with a slight roughness of the holes, which was probably due to either the dry or wet etching of the Cr solvent. 

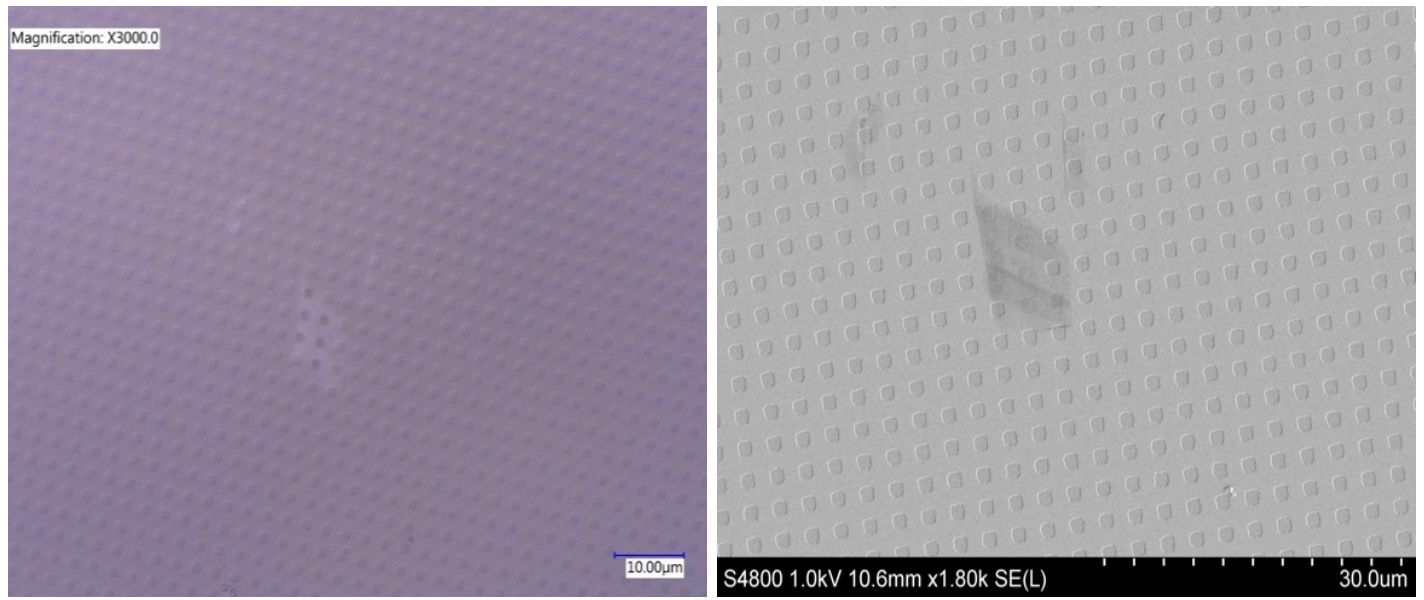

Figure 7.27: Suspended graphene on patterned mica substrate. Left: Optical image. Right: SEM

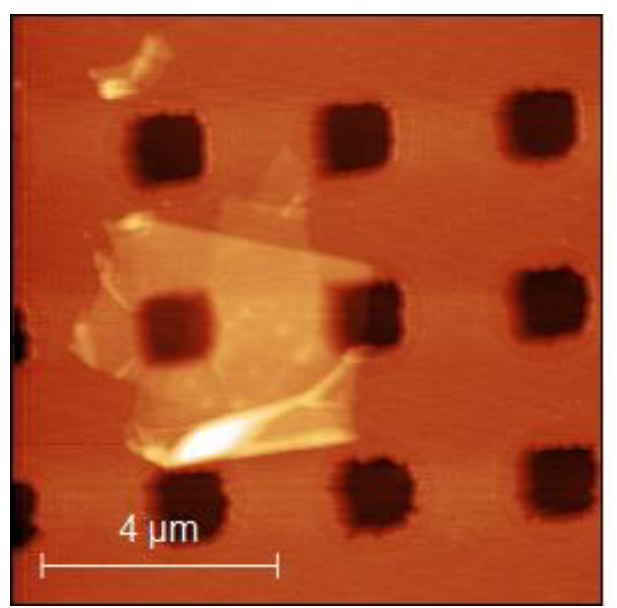

Figure 7.28: AFM image of suspended graphene sheet on the patterned mica substrate

\subsection{Conclusion}

Electron-beam lithography has become a common choice for device fabrication due to its versatile pattern shaping and high resolution at nearly $20 \mathrm{~nm}$. In addition, the fact that a physical mask is not required eliminates the time delays and costs associated with mask fabrication, which is required with photolithography. Dry etching is a 
preferable method over wet etching for fabricating structures that display different shapes and sizes with a high aspect ratio.

This work showed the fabrication processes of nanostructures with periodic designs of squares and holes, which were created using the Raith software, EBL and plasma dry etching. The patterned mica substrate fabrication process was repeated many times due to the many obstacles faced; some were solved, and others required some adjustment. However, finally, the mica was successfully etched, and the goal of this thesis was achieved.

The successful final method for the PL etching of mica - the first such reported method - was presented as mica/250 nm Cr/nLOF 2070. The $\mathrm{Cr}$ was used for two reasons: as a hard mask to protect the mica surface from the high power of plasma and to prevent charging and pattern deformation.

Cleaning the substrate before resist coating is important for reducing surface roughness, as it removes contamination; it also increases the resist homogeneity by eliminating contaminants and causing the resist adhesion to increase. Here, this was achieved by annealing to remove the water layer; due to the high humidity in the clean room, no further solvent treatments were required. Moreover, the baking time for negative resist nLOF 2070 was found be very important to stabilise the development process. Pre-exposure baking, i.e. the soft bake, improved the adhesion between the resist and the substrate, while the PEB, i.e. the hard bake, was needed in order to increase the thermal, chemical and physical stability of the developed resist structures. In order to reach the desired resist stability, two minutes for the pre- and post-exposure stages was the standard developing time. To recap the successful PL for the mica substrate, the exposure at $5 \mathrm{kV}$ acceleration voltage and a $60-\mu \mathrm{m}$ aperture with a maximum dose of $40 \mu \mathrm{C} / \mathrm{cm}^{2}$ produced the optimal e-beam exposure in this work. The pattern was developed in 2:1 AZ 726 MIF: DI water for one minute. The following step was opening windows in the $\mathrm{Cr}$ layer using the $\mathrm{Cr}$ etchant solvent. The resist was stripped for an hour in hot DMSO. Then, dry etching was performed with $\mathrm{CF}_{4}$ (50 $\mathrm{sccm}), \operatorname{Ar}(20 \mathrm{sccm}), 200 \mathrm{~W}$ power, $100 \mathrm{mT}$ Trr pressure and an etching time of 10 minutes. After that, the $\mathrm{Cr}$ stripper removed the remaining $\mathrm{Cr}$ layer. Finally, holes were created with a diameter of approximately $2 \mu \mathrm{m}$ and an etch depth of approximately 35 $\mathrm{nm}$. Good quality patterns were achieved with slight roughness of the holes, which was probably due to the etching process. 
This work has reported the first successful method for the PL etching of mica, which opens up the opportunity to develop and optimise the control of the etching rate for mica substrate. 


\subsection{References}

[1] Blake P, Brimicombe P, Nair R, Booth T, Jiang D, Schedin F, et al. Graphenebased liquid crystal device. Nano Letters. 2008;8(6): 1704-1708.

[2] Eda G, Fanchini G, Chhowalla M. Large-area ultrathin films of reduced graphene oxide as a transparent and flexible electronic material. Nature Nanotechnology. 2008;3(4): 270-274.

[3] Kim K, Zhao Y, Jang H, Lee S, Kim J, Ahn J, et al. Large-scale pattern growth of graphene films for stretchable transparent electrodes. Nature. 2009;457(7230): 706-710.

[4] Mak KF, Lee C, Hone J, Shan J, Heinz TF. Atomically thin MoS2: A new directgap semiconductor. Physical Review Letters. 2010;105(13): 136805.

[5] Splendiani A, Sun L, Zhang Y, Li T, Kim J, Chim CY, et al. Emerging photoluminescence in monolayer MoS2. Nano Letters. 2010;10(4): 1271-1275.

[6] Korn T, Heydrich S, Hirmer M, Schmutzler J, Schüller C. Low-temperature photocarrier dynamics in monolayer MoS2. Applied Physics Letters. 2011;99(10): 102109.

[7] Castellanos-Gomez A, Poot M, Steele GA, van der Zant HSJ, Agraït N, RubioBollinger G. Elastic properties of freely suspended MoS2 nanosheets. Advanced Materials. 2012;24(6): 772-775.

[8] Radisavljevic B, Radenovic A, Brivio J, Giacometti V, Kis A. Single-layer MoS2 transistors. Nature Nanotechnology. 2011;6(3): 147-150.

[9] Dean C, Young A, Meric I, Lee C, Wang L, Sorgenfrei S, et al. Boron nitride substrates for high-quality graphene electronics. Nature Nanotechnology. 2010;5(10): 722-726.

[10] Gorbachev R, Riaz I, Nair R, Jalil R, Britnell L, Belle B, et al. Hunting for monolayer boron nitride: Optical and Raman signatures. Small. 2011;7(4): 465468.

[11] Pacile D, Meyer J, Girit Ç, Zettl A. The two-dimensional phase of boron nitride: Few-atomic-layer sheets and suspended membranes. Applied Physics Letters. 2008;92(13): 133107. 
[12] Song L, Ci L, Lu H, Sorokin P, Jin C, Ni J, et al. Large-scale growth and characterization of atomic hexagonal boron nitride layers. Nano Letters. 2010;10(8): 3209-3215.

[13] Möller M, Handge U, Kunz D, Lunkenbein T, Altstädt V, Breu J. Tailoring shearstiff, mica-like nanoplatelets. ACS Nano. 2010;4(2): 717-724.

[14] Castellanos-Gomez A, Wojtaszek M, Tombros N, Agraït N, van Wees BJ, RubioBollinger G. Atomically thin mica flakes and their application as ultrathin insulating substrates for graphene. Small. 2011;7(17): 2491-2497.

[15] Fu YT, Zartman GD, Yoonessi M, Drummy LF, Heinz H. Bending of layered silicates on the nanometer scale: Mechanism, stored energy, and curvature limits. The Journal of Physical Chemistry C. 2011;115(45): 22292-22300.

[16] Gao J, Guo W, Geng H, Hou X, Shuai Z, Jiang L. Layer-by-layer removal of insulating few-layer mica flakes for asymmetric ultra-thin nanopore fabrication. Nano Research. 2012;5(2): 99-108.

[17] Low CG, Zhang Q. Ultra-thin and flat mica as gate dielectric layers. Small. 2012:8(14): 2178-2183. Available from: DOI: 10.1002/smll.201200300.

[18] Paul B, Lu J, Eklund P. Nanostructural tailoring to induce flexibility in thermoelectric $\mathrm{Ca} 3 \mathrm{Co} 4 \mathrm{O} 9$ thin films. ACS Applied Materials \& Interfaces. 2017;9(30): 25308-25316.

[19] Müller M, Fiedler T, Gröger R, Koch T, Walheim S, Obermair C, et al. Controlled structuring of mica surfaces with the tip of an atomic force microscope by mechanically induced local etching. Surface and Interface Analysis. 2004;36(2): $189-192$.

[20] Jiang Y, Gao J, Guo W, Jiang L. Mechanical exfoliation of track-etched twodimensional layered materials for the fabrication of ultrathin nanopores. Chemical Communications. 2014;50(91): 14149-14152.

[21] Liu Z, Brown N, McKinley A. Characterisation of oxygen plasma-modified mica surfaces using XPS and AFM. Applied Surface Science. 1997;108(3): 319-332.

[22] Brown N, Liu Z. The etching of natural alpha-recoil tracks in mica with an argon RF-plasma discharge and their imaging via atomic force microscopy. Applied Surface Science. 1996;93(2): 89-100. 
[23] Liu Z, Brown N. XPS characterization of mica surfaces processed using a radiofrequency (rf) argon plasma. Journal of Physics D: Applied Physics. 1998;31(15): 1771-1781.

[24] Singh M, Kaur N, Singh L. Morphology of heavy ions irradiated mica. Radiation Physics and Chemistry. 2010;79(12): 1180-1188.

[25] Dai L, Griesser H, Mau A. Surface modification by plasma etching and plasma patterning. The Journal of Physical Chemistry B. 1997;101(46): 9548-9554.

[26] van Assenbergh P, Meinders E, Geraedts J, Dodou D. Nanostructure and microstructure fabrication: From desired properties to suitable processes. Small. 2018;14(30): 1801989.

[27] Trivedi T, Roy A, Movva H, Walker E, Bank S, Neikirk D, et al. Versatile largearea custom-feature van der Waals epitaxy of topological insulators. ACS Nano. 2017;11(7): 7457--7467.

[28] MicroChemicals. Basics of microstructuring. Available from https://microchemicals.com/technical_information/softbake_photoresist.pdf [Accessed 31st August 2020].

[29] MicroChemicals. Hardbake of photoresist structures. Available from: https://www.microchemicals.com/technical_information/hardbake_photoresist. pdf [Accessed 31st August 2020].

[30] Herth E, Algré E, Tilmant P, Francois M, Boyaval C, Legrand B. Performances of the negative tone resist AZnLOF 2020 for nanotechnology applications. IEEE Transactions on Nanotechnology. 2012;11(4): 854-859.

[31] Altissimo M. E-beam lithography for micro-/nanofabrication. Biomicrofluidics. 2010;4(2): 026503 


\section{Chapter 8}

\subsection{Summary}

To investigate the electrical properties of 2D systems like graphene, or incorporate them into electronic switching or MEMS sensing devices, the nanomaterial must first be deposited onto an electrically insulating support. While $\mathrm{SiO}_{2}$ has most commonly been used, it has been found that graphene deposited on $\mathrm{SiO}_{2}$ mechanically conforms to the rough oxide surface, worsening its electronic behaviour. In contrast, it has been demonstrated that graphene is free of ripples when supported on an ultraflat substrate such as mica. Typically, graphene on $\mathrm{SiO}_{2}$ is five times rougher than that on mica. Any advances in understanding the use of mica as an insulating support to investigate graphene must be compared to $\mathrm{SiO}_{2}$, and this thesis compares both together.

To use mica as an alternative support for studying mechanically-exfoliated graphene, several key challenges remain. The preparation of the mica and $\mathrm{SiO}_{2}$ supports and the deposition of the graphene is critical and this was investigated in Chapter 4. Several methods were tested for cleanliness and the preparation of the mica substrate, including environmental effects. Thermal oxidation of silicon was tested, and the required exposure recipe determined. As an electrically insulating support using SEM is particularly challenging, and settings were tested and optimised for SEM, optical microscopy and AFM.

Annealing graphene is a key step to remove surface contamination for investigation and is used in device fabrication. Chapter 5 investigated the effects of vacuum annealing and substrate morphology on thin and thick few-layer graphene attached to mica and $\mathrm{SiO}_{2}$ substrates, using Raman spectroscopy, AFM and SEM. Pre and post annealing, mica provided defect-free graphene, due to the flatness of mica substrate; no remarkable strain or doping in graphene sheets on mica were detected. On the other hand, graphene deposited on $\mathrm{SiO}_{2}$ substrate did show defects. This is a key finding that validates the work of this thesis to investigate mica.

Annealing at low temperatures of $300{ }^{\circ} \mathrm{C}$ was sufficient to improve the quality of graphene on $\mathrm{SiO}_{2}$ and remove most of the disorder in the graphene sheet. Annealing at high temperatures induced a compressive strain in the graphene. The results indicate that the deformation in graphene sheet can be increased, altering the structure and 
electronic properties of the graphene. This impact of annealing and morphology must be considered in all graphene studies. This is consistent with previous work. Annealing graphene on mica however showed no change in defects; confirming that annealing can be carried out without degradation of the graphene - an important observation.

This chapter also observed 'disappearing graphene', where few-layer graphene on $\mathrm{SiO}_{2}$ can no longer be imaged with SEM after annealing. In the past other groups have attributed this observation to the graphene being blown off, or incorporated into the surface. However, using Raman it was confirmed that the graphene was still there. By using AFM aligned to other features, and averaging line profiles, it was determined that the 'disappeared' graphene was still detectable with AFM, although the conformation to the rough $\mathrm{SiO}_{2}$ surface meant the apparent height above the background had reduced. Although not part of the main work, this is an exciting novel observation.

At the end of Chapter 5 unexpected structural changes were observed in the mica surface around the deposited graphene after the final annealing step at $500{ }^{\circ} \mathrm{C}$. This degradation caused an apparent height change in graphene post annealing, although no defect or strain was present in the graphene sheets. As AFM was only carried out at the last stage, Chapter 6 investigated this effect by performing AFM on bare mica after every annealing step. It was found that the structure of the mica substrate changed with the temperature. These results show that a few particles appeared in the surface structure of muscovite mica at $400{ }^{\circ} \mathrm{C}$, and a noticeable increase of particles was present in some of the samples of annealed mica at $500^{\circ} \mathrm{C}$. These were determined to be potassium carbonate particles, produced from the mica structure itself, as annealing causes dehydroxylation in mica. Consideration could be given to whether the graphene sheet worked as a mask to protect the mica surface underneath, which would expand the field of graphene applications. For this work, it places an upper limit on annealing graphene on mica of $300^{\circ} \mathrm{C}$.

When mica is used an electrically insulating support for the study of nanomaterials like graphene, locating small samples repeatedly across several instruments is made easier when location grids or markers are etched into the substrate. Typically, these need to be deep enough to provide contrast in optical and electron microscopy but are not as deep as the typical trenches etched to create the voids used for suspending 
nanomaterials. Trenches or voids are required for devices which suspend graphene on the insulating support, for applications like sensing or MEMS devices. Chapter 7 developed a technique to pattern the mica substrate using e-beam lithography, which allows the later deposition of graphene samples to create suspended sheets over the holes. In this chapter, a variety of issues were overcome, and solutions were suggested. As part of the recipe development, photolithography was used as a faster technique to examine different pattering recipes.

A successful e-beam pattering recipe was finally established. This includes a chromium (Cr) layer on the top of mica, with many benefits, including improving adhesion, and avoiding deformation in the patterning from electron beam charging. It also protects the mica surface from plasma etching, reducing the roughness of the substrate. A negative resist mask was deposited on the top of the $\mathrm{Cr}$ layer, then the lithography process was performed. Next, dry plasma etching was examined at different parameters, including the radio frequency $(\mathrm{RF})$ power, pressure, gas flow and etching time. After all the deposited layer was removed using solvents, the resultant patterned mica was characterised using optical microscopy and AFM.

At the end of Chapter 7, exfoliated graphene was deposited successfully on patterned mica substrate. The suspended graphene sheets were characterised using the previously mentioned techniques. As far as the author is aware, this is the only demonstration of controlled lithographic etching of features into mica, and also of the successful deposition of graphene onto such structures. The thesis, in conclusion, now opens the way for researchers to controllably study the properties of graphene on patterned, insulating, mica supports.

\subsection{Future work}

Graphene conformation to $\mathrm{SiO}_{2}$ roughness worsens the electronic properties, whereas graphene deposited on flat terraces of insulating mica is free of ripples. This thesis has addressed several key challenges of using mica to support mechanically exfoliated graphene. Most significantly, patterning and etching mica to create location grids and etch trenches to suspend graphene is now possible. Future work can now perform electrical and electro-mechanical studies of both and supported and suspended 
graphene layers to investigate how the electrical properties differ to that of graphene suspended and supported on $\mathrm{SiO}_{2}$ substrates. The reduction in substrate-induced rippling is very likely to alter the conductivity of the graphene and it should increase with the reduction in charge scattering. This can be tested. Further, this thesis has concluded that graphene on mica should be annealed at $300^{\circ} \mathrm{C}$ to reduce contamination but avoid thermally-induced disorder. Any electrical and electro-mechanical studies should first anneal graphene on mica at this temperature.

Graphene on $\mathrm{SiO}_{2}$ appeared to become 'invisible' with $\mathrm{AFM}$ after annealing at $500^{\circ} \mathrm{C}$, in line with previous observations with scanning electron microscopy. Other studies attributed this to the graphene being removed, but, here, using substrate markers, Raman spectroscopy and line-averaged AFM showed that the graphene was still present but had conformed to the underlying roughness of the $\mathrm{SiO}_{2}$ so well as to appear nearly invisible. Future work can examine this effect for its potential applications in transparent conductors, or invisible transceiver use. The DC and AC electrical response should be measured to see whether conformed graphene can still operate electrically sufficiently to act as a conductor which is 'invisible' from the background material. The 'invisibility' is confirmed here mechanically by AFM, and at the wavelength of the SEM, but should be examined at optical wavelengths too.

Mica annealed at $400^{\circ} \mathrm{C}$ showed the formation of potassium carbonate particles following dehydroxylation of the mica surface at a temperature lower than previously reported. In addition, the graphene appeared to act as a mask, protecting the mica underneath it while the surrounding surface was removed at $500^{\circ} \mathrm{C}$. There is potential future work using graphene itself as a mask to etch and pattern mica. If patterned graphene-on-mica can act as a physical mask, it may be an alternative way to achieve deeper etches in mica and should be investigated. 